Florida International University FIU Digital Commons

9-17-2015

\title{
Comprehensive Analysis of Emerging New Psychoactive Substances by Ion Mobility Spectrometry and Mass Spectrometry
}

Seongshin Gwak

Florida International University, sgwak001@fiu.edu

DOI: $10.25148 /$ etd.FIDC000157

Follow this and additional works at: https://digitalcommons.fiu.edu/etd

Part of the Analytical Chemistry Commons

\section{Recommended Citation}

Gwak, Seongshin, "Comprehensive Analysis of Emerging New Psychoactive Substances by Ion Mobility Spectrometry and Mass Spectrometry" (2015). FIU Electronic Theses and Dissertations. 2291.

https://digitalcommons.fiu.edu/etd/2291 


\title{
FLORIDA INTERNATIONAL UNIVERSITY
}

Miami, Florida

\section{COMPREHENSIVE ANALYSIS OF EMERGING NEW PSYCHOACTIVE SUBSTANCES BY ION MOBILITY SPECTROMETRY AND MASS SPECTROMETRY}

\author{
A dissertation submitted in partial fulfillment of \\ the requirements for the degree of \\ DOCTOR OF PHILOSOPHY \\ in \\ CHEMISTRY \\ by
}

Seongshin Gwak 
To: $\quad$ Dean Michael R. Heithaus

College of Arts and Sciences

This dissertation, written by Seongshin Gwak, and entitled Comprehensive Analysis of Emerging New Psychoactive Substances by Ion Mobility Spectrometry and Mass Spectrometry, having been approved in respect to style and intellectual content, is referred to you for judgment.

We have read this dissertation and recommend that it be approved.

Anthony P. DeCaprio

Fenfei Leng

Wenzhi Li

Bruce R. McCord

José R. Almirall, Major Professor

Date of Defense: September 17, 2015

The dissertation of Seongshin Gwak is approved.

\begin{tabular}{r} 
Dean Michael R. Heithaus \\
College of Arts and Sciences \\
\hline Dean Lakshmi N. Reddi \\
University Graduate School
\end{tabular}

Florida International University, 2015 
(C) Copyright 2015 by Seongshin Gwak

All rights reserved. 


\section{DEDICATION}

I would like to dedicate this dissertation to my family and my fiancée for their endless love, support, and encouragement. 


\section{ACKNOWLEDGMENTS}

First of all, I would like to express my deepest gratitude to Dr. José Almirall, my advisor, for his support and guidance. He motivated me all the time of research during my doctoral career. I also would like to thank my dissertation committee, Dr. Anthony DeCaprio, Dr. Fenfei Leng, Dr. Wenzhi Li, and Dr. Bruce McCord, for their support and guidance, especially for taking time out of their busy schedules.

I would like to acknowledge the National Institute of Justice for the research grant (Award No. 2011-DN-BX-K531) and the University Graduate School of Florida International University for the Dissertation Year Fellowship. This research would not have been possible without their financial support. Thanks to the Senate Graduate and Professional Student Committee and the College of Arts and Sciences of Florida International University for travel funding to attend a number of scientific conferences throughout my doctoral study.

I would like to thank Dr. Luis Arroyo-Mora from the Forensic \& Analytical Toxicology Facility at Florida International University for his technical support and the use of the Agilent 6530 Q-TOF mass analyzer. I also appreciate Oliver Spicer, Jr. from the Analytical Section at Miami-Dade Police Department Forensic Services Bureau for providing the seized drug samples.

Many thanks to my fellow colleagues and friends whom I have met during my doctoral degree for their encouragement and support. I greatly appreciate all formal and present A-Team members for their help and sharing all the fun inside and outside of the laboratory. Thanks to all my friends outside of the laboratory for sharing all good memories during this long journey. 
Most importantly, I would like to thank my family in Korea for their spiritual support during my study abroad as well as through my entire life. Special thanks to my fiancée for her support, patience, and love. I am very grateful to have all of them in my life. 


\begin{abstract}
OF THE DISSERTATION
COMPREHENSIVE ANALYSIS OF EMERIGING NEW PSYCHOACTIVE

SUBSTANCES BY ION MOBILITY SPECTROMETRY AND MASS

SPECTROMETRY

by

Seongshin Gwak
\end{abstract}

Florida International University, 2015

Miami, Florida

Professor José R. Almirall, Major Professor

In the new era of drug abuse, the proliferation of new psychoactive substances (NPS), commonly referred to as designer drugs or legal highs, has been a global concern. These substances are produced to circumvent current legislation for controlled substances with minor modifications in their chemical structure. Although many efforts have been made previously, the characterization of such substances are still challenging because of (1) the continual emergence of newly identified substances, (2) the lack of a universal screening test for NPS that are structurally similar to each other, and (3) the complex and time-consuming chromatographic techniques currently used. Therefore, it is necessary to develop novel analytical methods that can be readily adapted by forensic laboratories to overcome these challenges.

In this dissertation, various analytical techniques have been evaluated for qualitative analysis of these emerging NPS. For rapid screening purposes, a commercial ion 
mobility spectrometry with a ${ }^{63} \mathrm{Ni}$ ion source $\left({ }^{63} \mathrm{Ni}-\mathrm{IMS}\right)$ and also direct analysis in real time coupled to a quadrupole time-of-flight mass spectrometer (DART-QTOF-MS) were investigated first. The results showed that rapid detection by ${ }^{63} \mathrm{Ni}-\mathrm{IMS}$ and identification by DART-QTOF-MS can be achieved with sub-nanogram detection capability and high speed total analysis time less than two minutes. In recent developments of gas chromatography mass spectrometry (GC-MS), gas chromatography (GC) has been coupled to state-of-the-art mass spectrometers, including triple quadrupole (MS/MS) and quadrupole time-of-flight (QTOF). It was revealed that the application of GC-MS/MS and GC-QTOF facilitates the unambiguous identification of emerging NPS with a chemical ionization (CI) source. In addition, constitutional isomers of NPS were differentiated with the capabilities of product ion scan and multiple reaction monitoring (MRM) modes. Finally, the coupling of IMS with a mass spectrometer (IMS-MS) was investigated as an alternative confirmatory technique. With the development of an optimal solvent system in the electrospray ionization (ESI) process, the rapid analysis and identification of synthetic cathinone was successfully achieved less than five minutes. As a proof-of-concept, seized drugs samples provided by a local forensic laboratory were analyzed using these developed methods by various analytical techniques. The results from these seized samples are also presented in this evaluation. 


\section{TABLE OF CONTENTS}

CHAPTER

PAGE

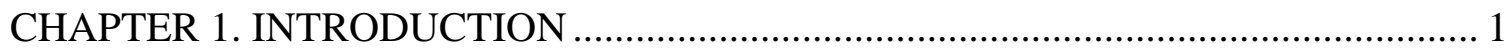

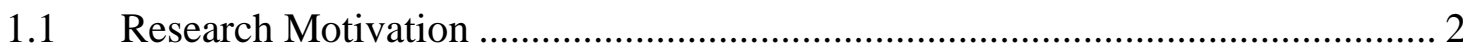

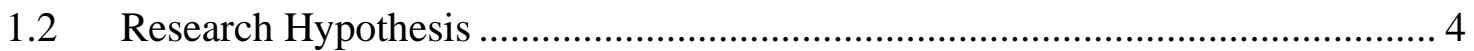

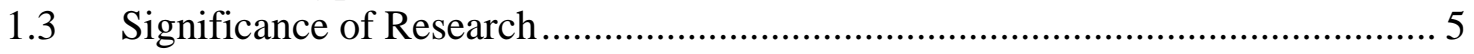

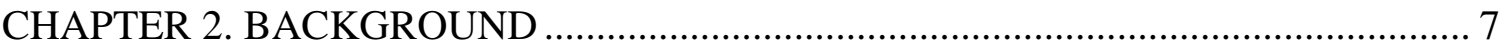

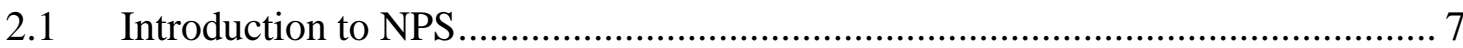

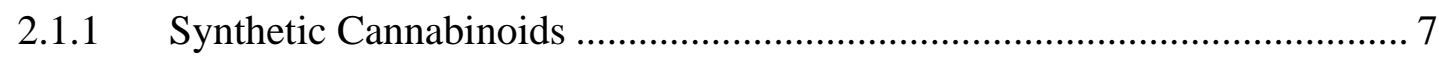

2.1.2 Synthetic Cathinones ………………………...................................... 8

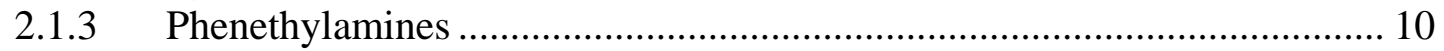

2.1.4 Ketamine and Phencyclidine-type Substances............................................. 11

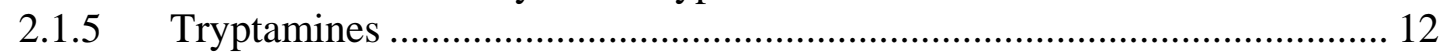

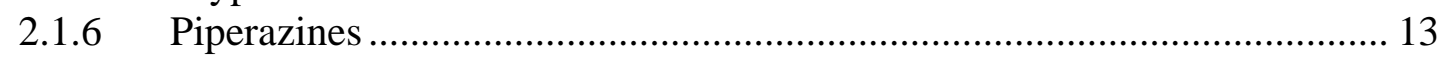

2.2 Forensic Analysis of Controlled Substances .................................................. 15

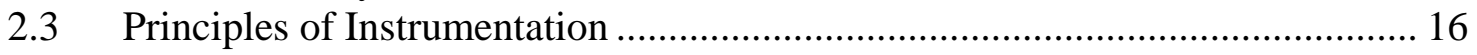

2.3.1 Gas Chromatography ……………………..................................... 16

2.3.2 Ion Mobility Spectrometry .................................................................... 18

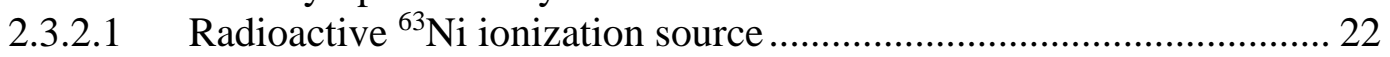

2.3.2.2 Coupling of Electrospray Ionization and Mass Spectrometry with Ion Mobility Spectrometry...................................................................... 23

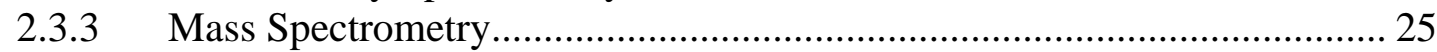

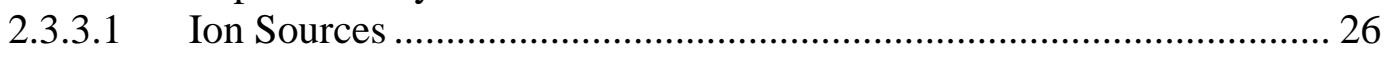

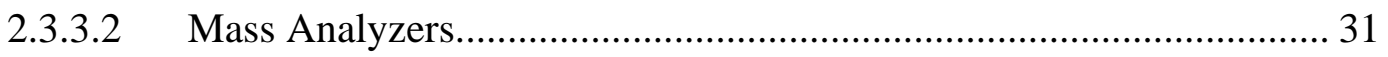

2.3.4 Direct Analysis in Real Time Mass Spectrometry ....................................... 38

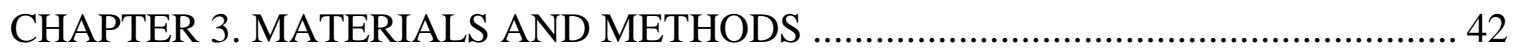

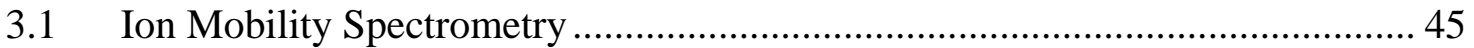

3.2 Direct Analysis in Real Time Quadrupole Time-of-Flight ................................. 46

3.3 Gas Chromatography Tandem Mass Spectrometry …………………………..... 46

3.4 Gas Chromatography Quadrupole Time-of-Flight........................................... 47

3.5 Electrospray Ionization Ion Mobility Spectrometry Mass Spectrometry........... 48

CHAPTER 4. DEVELOPMENT OF RAPID SCREENING METHODS FOR NPS...... 49

4.1 Qualitative Analysis of NPS by Ion Mobility Spectrometry............................. 49

4.1.1 Programing of Parameters and Detection of NPS....................................... 50

4.1.2 Determination of Detection Limits .......................................................... 54

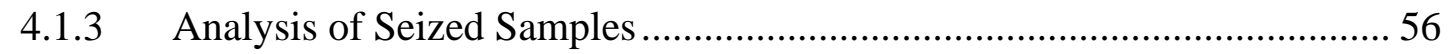

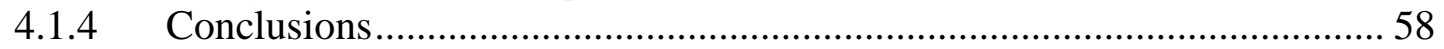

4.2 Direct Analysis in Real Time Quadrupole Time-of-Flight ................................59

4.2.1 Optimization of Parameters ……………………..................................... 60

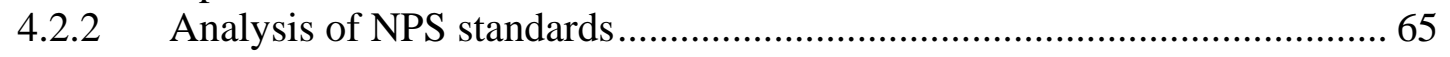


4.2.3 Determination of Detection Limits ................................................... 72

4.2.4 Analysis of Seized Samples ............................................................... 73

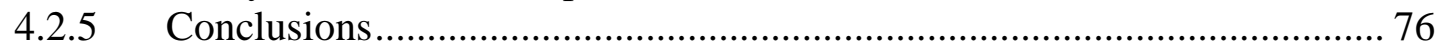

CHAPTER 5. DEVELOPMENT OF CONFIRMATORY METHODS FOR NPS ......... 77

5.1 Gas Chromatography Tandem Mass Spectrometry ........................................ 77

5.1.1 Retention-Time Locking and Multiple Reaction Monitoring Mode

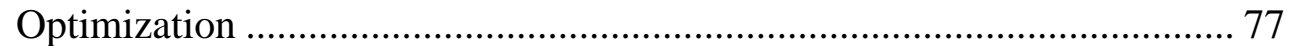

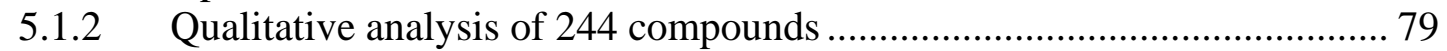

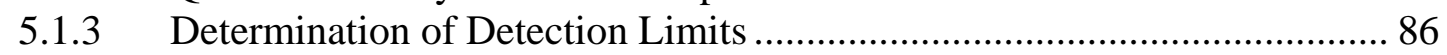

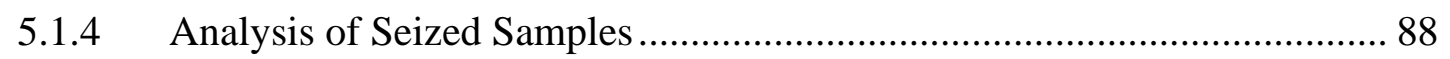

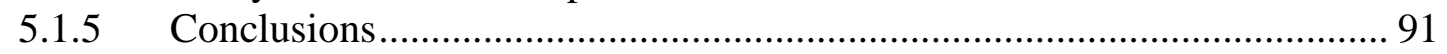

5.2 Gas Chromatography Quadrupole Time-of-Flight........................................ 92

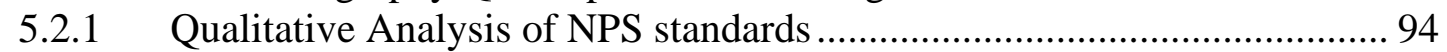

5.2.2 Determination of Detection Limits ....................................................... 97

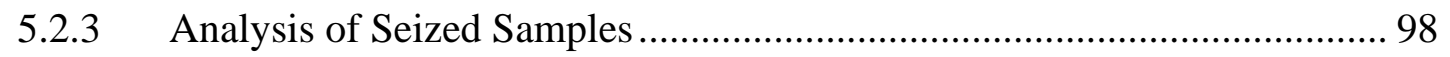

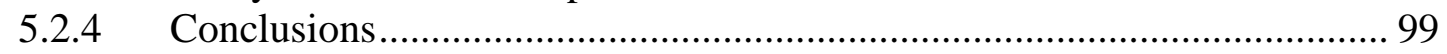

5.3 Electrospray Ionization Ion Mobility Spectrometry Mass Spectrometry......... 100

5.3.1 Optimization of Solvent System ........................................................... 102

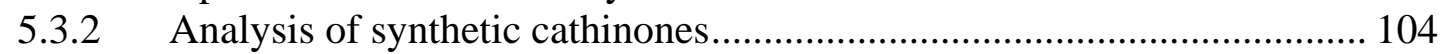

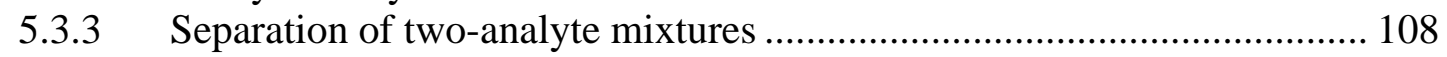

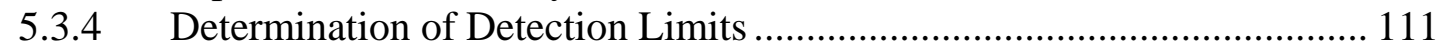

5.3.5 Analysis of Seized Samples ................................................................. 113

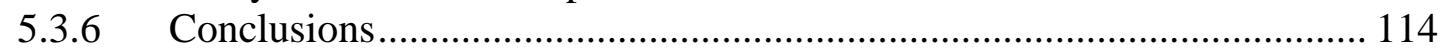

CHAPTER 6. RAPID DETECTION AND IDENTIFICATION OF FLAKKA ............ 115

6.1 Introduction to Flakka ............................................................................. 115

6.2 Rapid Detection and Characterization by Ion Mobility Spectrometry............ 116

6.3 Rapid Identification by Direct Analysis in Real Time Mass Spectrometry .... 117

6.4 Conclusions .......................................................................................... 119

CHAPTER 7. DISCUSSION AND OVERALL CONCLUSIONS ............................. 120

7.1 Rapid Screening Tests for NPS by IMS and DART-QTOF-MS ................... 120

7.2 Confirmatory Techniques for NPS by GC-MS and ESI-IMS-MS ................. 121

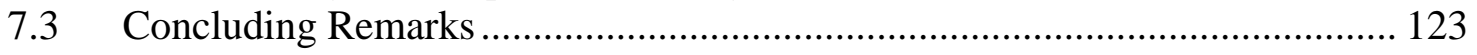

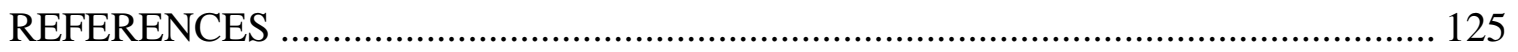

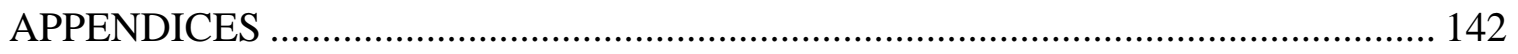

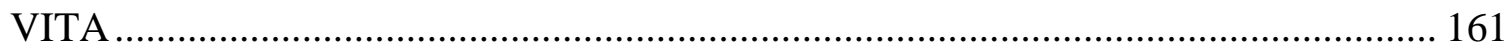




\section{LIST OF TABLES}

TABLE

PAGE

Table 1. Categories of analytical techniques suggested by SWGDRUG for the identification of controlled substances; adapted from [60] 15

Table 2. List of NPS standards that are prepared in dimethyl sulfoxide and ethanol....... 42

Table 3. List of solution based NPS standards with different solvents. ......................... 43

Table 4. Operating condition for the Barringer IONSCAN 400B............................... 45

Table 5. List of 35 NPS analyzed by the Barringer IMS and the results from the study

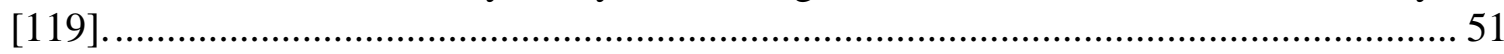

Table 6. Results of seized drug sample analysis by the Barringer IMS [119]................. 58

Table 7. List of 35 NPS analyzed by DART-QTOF-MS [119]. High mass accuracy

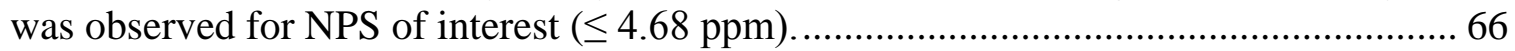

Table 8. Summary of major peaks in EI and CI full scan MS for the analytes of interest with the molecular ions, shown in bold. Molecular ions are absent for the highlighted

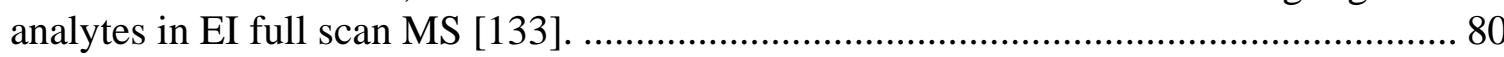

Table 9. Limits of detection (LODs) comparison between EI and CI for representative

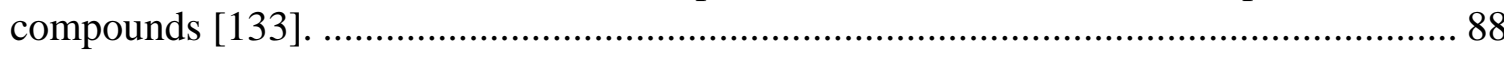

Table 10. List of additional 12 substances analyzed by GC-QTOF-MS........................ 95

Table 11. Ranges of quantifier and qualifier ions for CP-47,497 and JWH-250 during

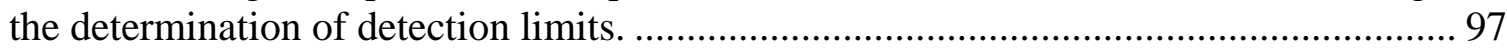

Table 12. List of 6 NPS analyzed by ESI-IMS-MS and the results from the study....... 105

Table 13. Comparison of various analytical techniques evaluated in this dissertation. . 123 


\section{LIST OF FIGURES}

FIGURE

PAGE

Figure 1. A number of NPS reported to the UNODC by Member States from 2009 to 2014; adapted from [9].... 3

Figure 2. Chemical structures of THC, CP 47,497, HU-210, JWH-018, and AM1220.... 8

Figure 3. Chemical structures of cathinone, 3-FMC, methylone, methedrone, 4-MEC,

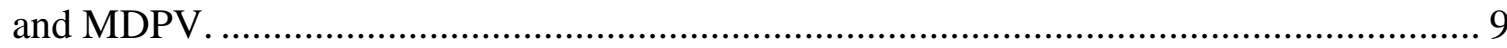

Figure 4. Chemical structures of mescaline, DOI, 2C-I, and 25I-NBOMe................... 11

Figure 5. Chemical structures of phencyclidine, ketamine, and methoxetamine. ........... 12

Figure 6. Chemical structures of 5-HT, AMT, and 5-MeO-DMT................................ 13

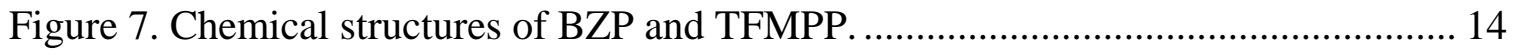

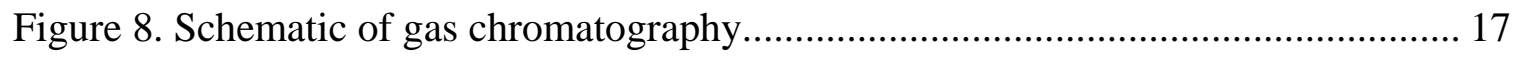

Figure 9. Schematic of a commercial IMS instrument with a ${ }^{63} \mathrm{Ni}$ radioactive ionization source and an example of ion mobility spectrum; Adapted and reproduced from [68]. .. 19

Figure 10. Proposed schematic of the interface close-up between an ion mobility spectrometer and a mass spectrometer; adapted from [82]........................................ 24

Figure 11. Diagram of an electron ionization source; adapted and reproduced from [84]

Figure 12. Diagram of a chemical ionization source.

Figure 13. Formation of a Taylor cone in the process of positive ion production in the electrospray ionization; reproduced and modified from [84].

Figure 14. Diagram of a quadrupole mass analyzer with hyperbolic rods and applied potentials; reproduced and modified from [84].

Figure 15. Symbolism for the representation of four different scan modes as proposed by Cooks et al.; adapted and modified from [84]. 35

Figure 16. Diagram of a triple quadrupole mass analyzer. 36

Figure 17. Schematic of a commercial high resolution quadrupole time-of-flight mass spectrometer; reproduced and modified from [92]. 
Figure 18. Schematic of the DART ionization source; reproduced and modified from [98].

Figure 19. Picture of Barringer IONSCAN 400B.

Figure 20. Overlaid ion mobility spectra of the representative NPS by the Barringer IMS [119]. Each substance was distinctly detected with a different drift time and a consistent reactant ion peak (RIP).

Figure 21. Ion mobility spectra of the two mixtures (a) 3-FMC, methylone, and MDPV, and (b) 4- MMC, butylone, and MDPV at the concentration of $3.3 \mu \mathrm{g} / \mathrm{mL}$ each [119]. The baseline separation was observed when analytes have significantly different $K_{0}$ values only.

Figure 22. Calibration curves for bk-EBDB (top), bk-MDDMA (middle), and APICA (bottom) in three different days by ${ }^{63} \mathrm{Ni}$-IMS. Each curve showed a good linearity in the linear range of 100 to $500 \mathrm{pg}$.

Figure 23. Overlaid ion mobility spectra of four seized drug samples provided by a local forensic laboratory [119]. It was found that blind sample 3 contains multiple compounds.

Figure 24. The DART source coupled to a QTOF mass spectrometer with a linear rail system for the sample introduction.

Figure 25. Optimization of the fragmentor voltage for the QTOF-MS. The ideal voltage was $250 \mathrm{~V}$ for both compounds between replicates.

Figure 26. Sample introduction by 1D transmission module using the linear rail system.

Figure 27. Different types of insulating caps: (a) flat cap and (b) angle cap................... 64

Figure 28. Optimized operating conditions for (a) the sample speed and amount of sample delivered, (b) different positions for sample delivery, (c) different types of insulating cap, and (d) distance between the DART source and the inlet of MS.

Figure 29. Full scan MS obtained using DART-QTOF-MS, (a) $\alpha$-PVP, (b) bkMDDMA, and (c) XLR-11 [119]. The protonated molecular ion for each compound was observed as a base peak.

Figure 30. Product ion MS of bk-MDEA (left) and bk-MDDMA (right) at different collision energies of 10, 20, and $40 \mathrm{eV}$ [119]. Two constitutional isomers showed different fragmentation at $\mathrm{CE}$ of $20 \mathrm{eV}$....

Figure 31. Product ion MS of a bk-MDEA and bk-MDDMA mixture at CE of $20 \mathrm{eV}$. Characteristic peaks from each compound were identified in the mixture analysis. 
Figure 32. Calibration curves for bk-EBDB (top), bk-MDDMA (middle), and APICA (bottom) in three different days by DART-QTOF-MS.

Figure 33. Product ion scan mass spectra of seized drug samples at different collision energies [119]

Figure 34. EI full scan mass spectra for (a) JWH-251, (b) JWH-250, and (c) JWH-249 [133]. Unambiguous identification of these compound is challenging in EI full scan mode because of the mass spectral similarity.

Figure 35. EI full scan mass spectra for (a) CPE, (b) AM2233, and (c) AM1220 [133].

Similar mass spectra were observed for these compounds in EI full scan mode.

Figure 36. CI full scan mass spectra for (a) JWH-251, (b) JWH-250, and (c) JWH-249 [133]. The protonated molecular ion from each compound were observed with the characteristic ethyl adduct in CI full scan mode.

Figure 37. CI MRM mass spectra for the regioisomers of synthetic cannabinoids:

JWH-250, JWH-302, and JWH-201 [133]. 86

Figure 38. Four-point calibration curves for JWH-250 from GC-MS/MS with (a) EI and (b) CI using the software MassHunter Quantitative Analysis. White points were excluded from constructing calibration curves because of the disqualification for $\mathrm{S} / \mathrm{N}$ of three.

Figure 39. Mass spectra of seized sample by EI full scan (top), CI full scan (middle), and CI MRM (bottom).

Figure 40. Mass spectra of methylone by EI full scan (top), CI full scan (middle), and CI MRM (bottom).

Figure 41. Picture of GC-QTOF-MS system.

Figure 42. Schematic of a high resolution QTOF mass analyzer for GC-MS. Adapted and reproduced from [140].

Figure 43. Picture of removable ion source apparatus.

Figure 44. Summary of results from (a) synthetic cannabinoids and (b) additional types of NPS analyzed by GC-QTOF-MS in EI full scan.

Figure 45. Calibration curves for CP-47,497 and JWH-250 by GC-QTOF-MS with the EI source in three different days.

Figure 46. Chromatograms and mass spectra of two seized drug samples by GC-QTOF-MS in EI full scan mode. 
Figure 47. Excellims ESI-IMS-MS (RA4100).

Figure 48. An air-cooled ESI source used in the Excellims ESI-IMS-MS................... 102

Figure 49. Close-up of a cooled ESI source coupled to the IMS.

102

Figure 50. Optimization of elctrospray ionization solvent system for three different groups of synthetic cathinones. The consistent average intensity was observed the solvent composition of 80:20 with $2.5 \%$ FA for three compounds.

Figure 51. Overlaid ion mobility spectra of 6 synthetic cathinones by direct infusion.

Each substance was successfully detected by ESI-IMS-MS. 106

Figure 52. Full scan mass spectra of 6 synthetic cathinones by ESI-IMS-MS. Each substance was identified with the presence of a protonated molecular ion.

Figure 53. Ion mobility spectrum (top) and mass spectrum (bottom) of a mixture of 4-MMC and methedrone by ESI-IMS-MS. The presence of two compounds in the mixture was confirmed with protonated molecular ions.

Figure 54. Ion mobility spectra and full scan mass spectra of two mixtures with different relative concentration. Charge competition between methylone and MDPV were observed in mixture analysis.

Figure 55. Ion mobility spectra of mixtures with a different ratio of two compounds in concentration.

Figure 56. Calibration curves of six synthetic cathinones by ESI-IMS-MS. A good linearity was shown for each substance in different linear ranges.

Figure 57. Ion mobility spectrum (top) and corresponding mass spectrum (bottom) of the seized drug sample by ESI-IMS-MS. The seized drug sample was found to be methylone with the presence of protonated molecular ion.

Figure 58. Chemical structures of alpha-PVP, 4-MePBP, alpha-PBP, and alpha-PPP. 116

Figure 59. Overlaid ion mobility spectra of $\alpha$-PVP and 4-MePBP. Both NPS were successfully detected with its characteristic mobility and a consistent reactant ion peak (RIP).

Figure 60. Full scan and product ion scan mass spectra of (a) $\alpha$-PVP and (b) 4-MePBP obtained using DART-QTOF-MS. Differentiation between two compounds can be achieved with product ion scan mass spectra at $\mathrm{CE}$ of $20 \mathrm{eV}$. 


\section{LIST OF ACRONYMS AND ABBREVIATIONS}

\begin{tabular}{|c|c|}
\hline${ }^{63} \mathrm{Ni}$ & Nickel-63 \\
\hline AAPCC & American Association of Poison Control Centers \\
\hline APCI & Atmospheric Pressure Chemical Ionization \\
\hline BIP & Background Ion Peak \\
\hline $\mathrm{ACN}$ & Acetonitrile \\
\hline $\mathrm{CI}$ & Chemical Ionization \\
\hline CID & Collision-Induced Dissociation \\
\hline DART & Direct Analysis in Real Time \\
\hline DESI & Desorption Electrospray Ionization \\
\hline DMSO & Dimethyl Sulfoxide \\
\hline EI & Electron Ionization \\
\hline ESI & Electrospray Ionization \\
\hline $\mathrm{EtOH}$ & Ethanol \\
\hline FAB & Fast Atom Bombardment \\
\hline FD & Field Desorption \\
\hline FI & Field Ionization \\
\hline FTIR & Fourier Transform Infrared Spectroscopy \\
\hline GC & Gas Chromatography \\
\hline ICR & Ion Cyclotron Resonance \\
\hline IMS & Ion Mobility Spectrometry \\
\hline IT & Ion Trap \\
\hline$K$ & Mobility Coefficient \\
\hline$K_{0}$ & Reduced Mobility \\
\hline $\mathrm{LC}$ & Liquid Chromatography \\
\hline LOD & Limit of Detection \\
\hline MALDI & Matrix-Assisted Laser Desorption Ionization \\
\hline
\end{tabular}




\begin{tabular}{|c|c|}
\hline MDA & 3,4-Methylenedioxyamphetamine \\
\hline MDMA & 3,4-Methylenedioxymethamphetamine \\
\hline $\mathrm{MeOAc}$ & Methyl Acetate \\
\hline $\mathrm{MeOH}$ & Methanol \\
\hline MRM & Multiple Reaction Monitoring \\
\hline MS & Mass Spectrometry \\
\hline $\mathrm{MS} / \mathrm{MS}$ & Tandem Mass Spectrometry \\
\hline$m / z$ & Mass-to-Charge Ratio \\
\hline NPS & New Psychoactive Substances \\
\hline OT & Orbitrap \\
\hline PD & Plasma Desorption \\
\hline QqQ & Triple Quadrupole Mass Spectrometer \\
\hline QTOF & Quadrupole Time-of-Flight \\
\hline RIP & Reactant Ion Peak \\
\hline RTL & Retention-Time Locking \\
\hline $\mathrm{S} / \mathrm{N}$ & Signal-to-Noise Ratio \\
\hline SIMS & Secondary Ion Mass Spectrometry \\
\hline SPME & Solid Phase Microextraction \\
\hline SRM & Selected Reaction Monitoring \\
\hline SWGDRUG & Scientific Working Group for the Analysis of Seized Drugs \\
\hline THC & Delta-9-Tetrahydrocannabinol \\
\hline TLC & Thin Layer Chromatography \\
\hline TOF & Time-of-Flight \\
\hline UNODC & United Nations Office On Drugs And Crime \\
\hline
\end{tabular}




\section{CHAPTER 1. INTRODUCTION}

New psychoactive substances (NPS) have been a global concern in the new era of drug abuse with the continual emergence of newly identified substances. The European Community coined the term 'NPS' in 2005 [1]. As a broad term, it refers to substances of abuse that are not regulated under the 1961 United Nations Single Convention on Narcotic Drugs or the 1971 United Nations Convention on Psychotropic Substances, but are considered to pose a risk to public health [2]. The NPS are also commonly referred to as 'designer drugs', 'legal highs', 'research chemicals', 'herbal incenses', or 'bath salts' [3]. The United Nations Office on Drugs and Crime (UNODC) classifies NPS into nine sub-groups as follows: synthetic cannabinoids, synthetic cathinones, phenethylamines, piperazines, aminoindanes, ketamine and phencyclidine-type substances, plant-based psychoactive substances and other substances for those compounds that do not fall into these eight categories [3].

Although many such substances were first synthesized and patented many years ago, the exceptional increase in availability and abuse of NPS has been proliferated recently with advertisement and sale mainly through the Internet $[2,4]$. Some early examples of substances that could today be considered 'designer drugs' are 3,4methylenedioxyamphetamine (MDA) and 3,4-methylenedioxymethamphetamine (MDMA), which were first synthesized in the 1910s, and have been widely abused since the beginning of 1970s [5]. Most of the phenethylamines and tryptamines that are found on the illicit substance markets were first synthesized by Alexander Shulgin and Ann Shulgin, with their published work influencing the proliferation of these substances [6-7]. 
Phenethylamines also include a large number of psychedelic substances, including paramethoxymethamphetamine (PMMA), 2, 3-dimethoxy-4-iodophenethylamine (2C-I), and 2, 5-dimethoxy-4-iodoamphetamine (DOI). One of the most problematic NPS groups is synthetic cathinones, also called bath salts. In some other classification of NPS, synthetic cathinones are classified in the group of phenethylamines with the ketone functional group substituted at the beta carbon. As the most widely abused NPS, synthetic cannabinoids are often found in a mixture with herbal products under the brand name, "Spice" or "K2". These three groups of NPS are the most frequently reported to the UNODC while other groups are also well explained in the World Drug Report 2013 and 2014 by the UNODC [3, 8]. More background on these emerging NPS are described in section 12.1 .

\subsection{Research Motivation}

By the end of 2014, a total number of NPS reported to the UNODC early warning advisory by Member States were 541, which is more than three times compared to the report of 166 NPS in 2009 [8-9]. Of 541 NPS, 450 NPS were reported in 2014 while 69 NPS out of 450 were reported for the first time in 2014 as shown in Figure 1 [9]. In the United States, 4,261 calls were reported to the American Association of Poison Control Centers (AAPCC) for exposure to synthetic marijuana (e.g., synthetic cannabinoids) and bath salts (e.g., synthetic cathinones) in 2014 [10-11]. As a result of recent federal and state regulations, reported exposures to these substances have significantly decreased compared to the 13,105 exposures reported in 2011 [10-11]. However, the emergence of newly identified psychoactive substances still continues to cause serious concern 
worldwide [12-13]. The prevalence of NPS primarily affects young adults as these substances are assumed safer than other controlled substances and easily obtained through the Internet, convenience stores, and gas stations [14-16].

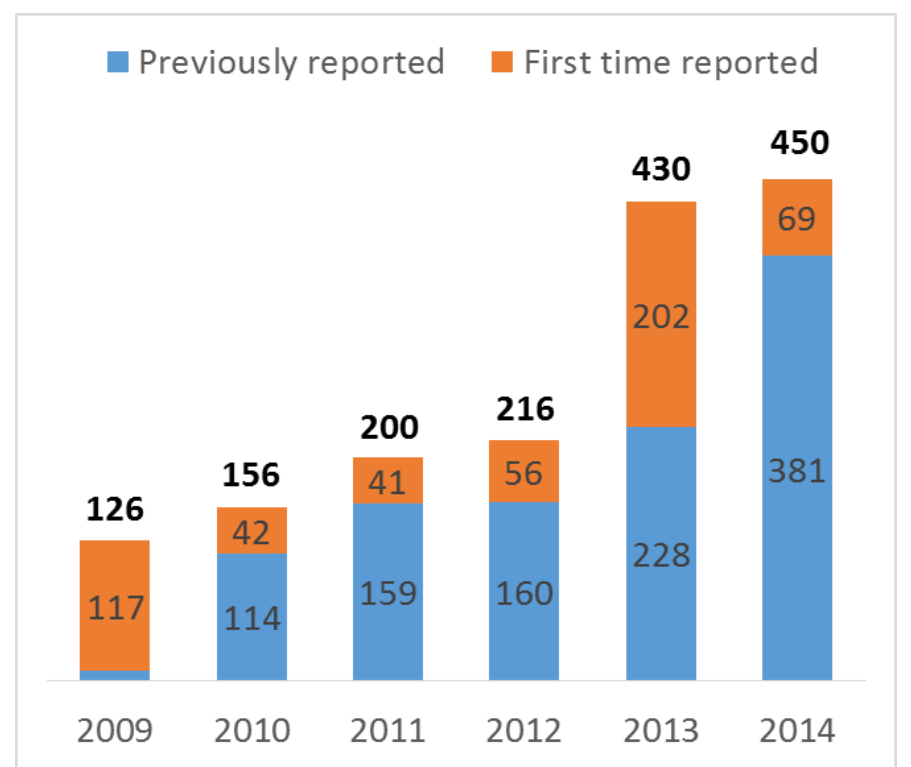

Figure 1. A number of NPS reported to the UNODC by Member States from 2009 to 2014; adapted from [9].

In the forensic analysis of drugs, color tests and immunoassay are commonly used for presumptive tests, and hyphenated chromatography-mass spectrometry is widely used for confirmatory tests [17]. Many efforts have been made to characterize and identify these emerging substances using gas chromatography-mass spectrometry (GC-MS) [1823] and liquid chromatography-mass spectrometry (LC-MS) [21, 24-27]. In addition, cross-reactivity of designer drugs in commercial immunoassay reagents has been studied comprehensively [28]. However, the rapid detection and unambiguous identification of such substances are still challenging because of (1) the continuous emergence of NPS, (2) the lack of a universal screening test for NPS that are structurally similar to each other, 
and (3) the complex and time-consuming chromatographic techniques currently used. To address these challenges, the current research aims to investigate the use of alternative state-of-the-art analytical techniques for the qualitative analysis of NPS at the level of seized drug analysis. The use of ion mobility spectrometry (IMS), direct analysis in real time quadrupole time-of-flight mass spectrometry (DART-QTOF-MS), gas chromatography tandem mass spectrometry (GC-MS/MS), electrospray ionization ion mobility spectrometry mass spectrometry (ESI-IMS-MS), and gas chromatography quadrupole time-of-flight mass spectrometry (GC-QTOF-MS) were applied to the detection, characterization, and/or identification of NPS. The utilization of IMS, a system currently deployed in many security checkpoints with portability capabilities, and DART-QTOF-MS will be used as a screening tool and provide resolution for rapid detection and identification of these emerging NPS. The use of GC-MS/MS, GC-QTOFMS, and ESI-IMS-MS is also evaluated as a confirmatory technique to provide unambiguous identification and characterization of NPS.

\subsection{Research Hypothesis}

Westphal et al. and Zaitsu et al. have shown the differentiation capability of phenethylamine and synthetic cathinone positional isomers by GC-MS/MS with the use of product ion scan mode $[20,22,29]$. On the basis of these findings, it is hypothesized that GC-MS/MS will facilitate the discrimination of other isomers that have not been reported in the literature as this instrument allows the elucidation of structure and determination of fragmentation with the product ion scan capability. 
Furthermore, product ion scan mass spectra generated from DART-QTOF will also allow the differentiation of isomers of interest. Musah et al. have shown the employment of collision-induced dissociation (CID) for the characterization of various cannabinoid analogs to provide confirmatory structural information [30]. Lesiak et al. investigated to differentiate between five structural isomers of synthetic cathinones with the implementation of in-source CID using DART-TOF [31]. Therefore, it is hypothesized that the differentiation of other structural isomers will be possible with the acquisition of product ion mass spectra using DART-QTOF-MS. In addition, the comparison of resulted product ion mass spectra between different ionization sources will be investigated with the assumption that the same protonated species will be produced from both the DART and electrospray ionization (ESI) sources.

Finally, previous studies have shown that ESI-IMS-MS allows for fast separation and detection of controlled substances, such as opiates and amphetamines [32-34]. In addition, an appropriate solvent system has been extensively investigated for the efficiency of ESI ionization by Holness et al. [35]. Hence, it is expected that comprehensive analysis will be possible for these emerging NPS by ESI-IMS-MS with the use of an optimal solvent system and the introduction of a secondary modifier.

\subsection{Significance of Research}

The current dissertation involves the investigation of a variety of NPS, mainly including synthetic cannabinoids, synthetic cathinones, phenethylamines, ketamine and phencyclidine-type substances, tryptamines, and piperazines, to aid rapid characterization and unambiguous identification. A commercial bench-top IMS with a radioactive nickel- 
$63\left({ }^{63} \mathrm{Ni}\right)$ ion source and DART-QTOF are attractive alternatives to the current screening methods with the rapid characterization capability of the emerging NPS. Gas chromatography interfaced with a triple quadrupole tandem mass spectrometer (GCMS/MS) and a quadrupole time-of-flight (GC-QTOF) as well as a commercial high resolution IMS coupled to a quadrupole mass spectrometer (IMS-MS) will be used for the unambiguous identification of these emerging substances.

Unambiguous fragmentation of isomers and the chemical structures of these emerging designer drugs are elucidated using multiple reaction monitoring (MRM) mode in GC-MS/MS as well as product ion scan mode in GC-MS/MS, GC-QTOF-MS and DART-QTOF-MS. Ultimately, the construction of a database for NPS by these state-ofthe-art analytical techniques will be useful for the identification of unknown analytes commonly encountered in forensic laboratories. In addition, the developed method for emerging NPS of interest in ESI-IMS-MS with the use of a soft ESI source will enable a fast and selective analysis, direct introduction of liquid samples, and the preservation of molecular ions for definitive identification. 


\section{CHAPTER 2. BACKGROUND}

\subsection{Introduction to NPS}

\subsubsection{Synthetic Cannabinoids}

The street names for synthetic marijuana or herbal marijuana alternatives are ' $\mathrm{K} 2$ ' and 'Spice', which are the legal form of marijuana that are sold via the Internet, local head shops, and convenience stores [36-37]. Synthetic marijuana is produced by spraying a solution of synthetic cannabinoids onto plant materials including, but not limited to Damiana shrub (Turnera diffusa), Ocimum basilicum, Mentha spicata, and Coriandrum sativum, in order to have a natural appearance similar to authentic herbal products $[36,38]$. In marijuana, delta-9-tetrahydrocannabinol (THC) is the main active component that produces the psychoactive effects [37]. There are different types of synthetic cannabinoids that mimic the effects of THC. For therapeutic purposes, John W. Huffman first synthesized a number of novel cannabinoids, called JWH series, which are abbreviated from the name of inventor [39]. Other common types of synthetic cannabinoids include HU-210 synthesized by Raphael Mechoulam, cyclohexylphenol (CP) cannabinoids synthesized by Pfizer, and AM series synthesized by Alexandros Markriyannis [37]. The chemical structures of these synthetic cannabinoids are given in Figure 2. 

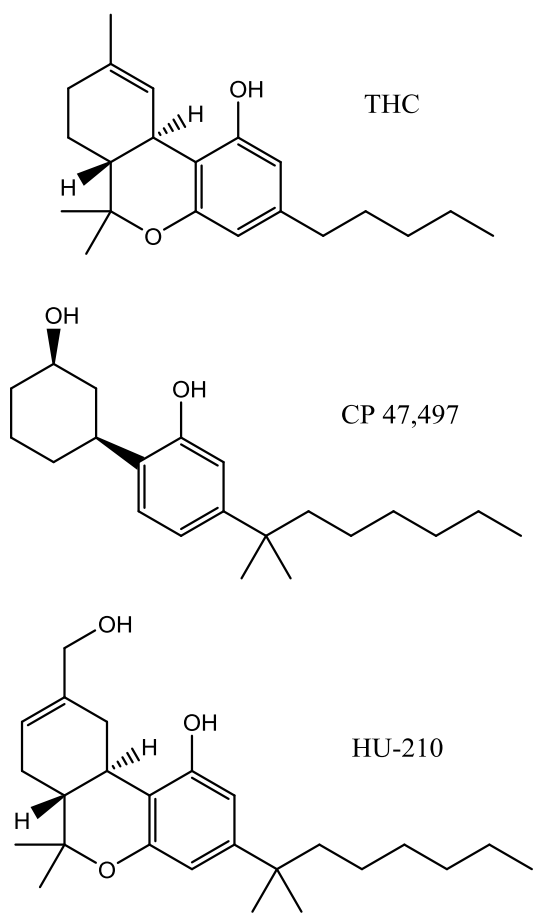

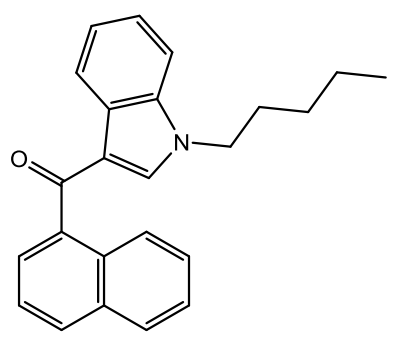

JWH-018

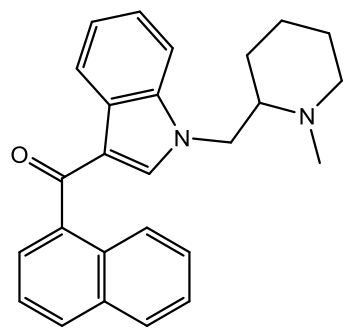

AM1220

Figure 2. Chemical structures of THC, CP 47,497, HU-210, JWH-018, and AM1220.

Although the pharmacology of synthetic cannabinoids in humans is not fully understood, various studies have shown that these synthetic cannabinoids act as cannabimimetic substances which bind the cannabinoid receptors 1 and $2\left(\mathrm{CB}_{1}\right.$ and $\left.\mathrm{CB}_{2}\right)$ [40].

\subsubsection{Synthetic Cathinones}

Synthetic cathinones are known as amphetamine-like stimulants producing psychoactive effects on the central nervous system [41]. As shown in Figure 3, the synthetic cathinone class of NPS has a similar backbone structure to cathinone, a natural compound present in the khat (Catha edulis) plant, for example, 3-fluoromethcathinone (3-FMC), 3,4-methylenedioxy-N-methylcathinone $\quad$ (methylone), methoxymethcathinone (methedrone), 4-methylethcathinone (4-MEC), and 3,4methylenedioxypyrovalerone (MDPV). The individual or mixtures of different synthetic 
cathinones are often called 'bath salts' and sold as white powder through the Internet, local head shops, and convenience stores [42]. Bath salts can be administered in various ways including inhalation, oral ingestion, injection, smoking, and snorting [42]. Although few studies have been conducted on the pharmacology of synthetic cathinones, it has been reported that the effects of these drugs are similar to cocaine, amphetamine, and MDMA [43]. The stimulating effect of synthetic cathinones is caused by the inhibition of norepinephrine and dopamine re-uptake [43]. The toxicity of these type of substances deposited onto both dopamine and serotonin neurons may result in unfortunate deaths [44]. Previous studies have shown that alphapyrrolidinopentiophenone ( $\alpha-\mathrm{PVP})$ is a potent dopamine and norepinephrine transporter inhibitor, but has relatively little affinity for the serotonin transporter [45]. In addition, it has also been revealed that 4-MEC inhibits the reuptake of dopamine transporter, norepinephrine transporter, and serotonin transporter but also acts as a 5hydroxytrypamine releaser [45].
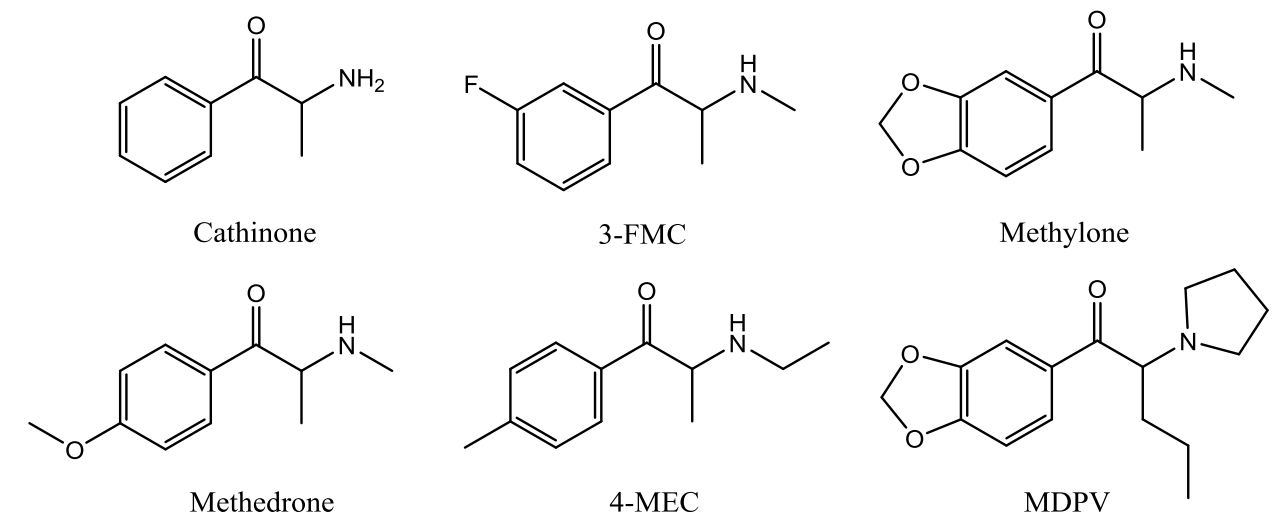

Figure 3. Chemical structures of cathinone, 3-FMC, methylone, methedrone, 4-MEC, and MDPV. 


\subsubsection{Phenethylamines}

As with other subgroups of NPS, many of the phenethylamines were introduced by Alexander and Ann Shulgin in their book, 'PiHKAL: A Chemical Love Story'. The book detailed a variety of psychedelic substances, including two carbon phenethylamine homologues (2C series), dimethoxy phenylisopropylamine (DO series), and 2C substances with a 2-methoxybenzyl group on the nitrogen (NBOMe series) as shown in Figure 4 [46-49]. These substances are in the same class as mescaline (peyote), which is a naturally occurring drug that produces hallucinogenic effects similar to lysergic acid diethylamide (LSD) [50]. A common route of phenethylamine administration is oral ingestion, while other routes of ingestion, including intravenous and intramuscular injections, insufflation, smoking, and rectal use have been also reported [51]. Although there is a limited amount of published scientific literature, most phenethylamines are known as serotonergic agonists acting on serotonin receptors $\left(5-\mathrm{HT}_{2}\right)$ with hallucinogenic effects [46-47]. Recent studies have revealed that the NBOMe series and 2C series are potent to serotonergic 5-hydroxytryptamine-2A $\left(5-\mathrm{HT}_{2} \mathrm{~A}\right), 5-\mathrm{HT}_{2 \mathrm{~B}}, 5-\mathrm{HT}_{2 \mathrm{C}}$ receptors and trace amine-association receptor 1 (TAAR1) [52]. 


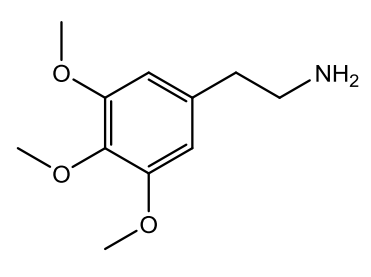

Mescaline<smiles>COc1cc(CC(C)N)c(OC)cc1I</smiles>

DOI

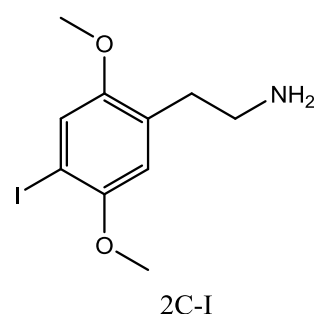

$2 \mathrm{C}-\mathrm{I}$<smiles>COc1cc(CCNCc2ccccc2OC)c(OC)cc1I</smiles>

25I-NBOMe

Figure 4. Chemical structures of mescaline, DOI, 2C-I, and 25I-NBOMe.

\subsubsection{Ketamine and Phencyclidine-type Substances}

Ketamine is also considered a popular drug of abuse in the category of NPS by UNODC [3]. Street names for ketamine include 'K', 'special K', 'kit kat', and 'super K', and it was initially synthesized as a derivative of phencyclidine (PCP). The primary use of ketamine was for the generation of anesthesia in both veterinary and human medicines [53]. It is also often misused as a club drug as a result of hallucinogenic effects it imparts on the user. However, in recent years, methoxetamine (MXE) has gained popularity due to the higher intensity of effects and the longer duration of action [53]. Hondebrink et al. reported that MXE was one of the most commonly submitted NPS in the Netherlands in 2013 along with 4-bromo- 2,5-dimethoxyphenethylamine (2C-B), 4-fluoroamphetamine (4-FA), and 6-(2- aminopropyl)benzofuran (6-APB) [54]. As a structural analogue of ketamine, MXE is often called 'Mexxy', 'M-ket', 'special M', or ' $\mathrm{K}_{\max }$ ' and sold on the 
streets as a free base and hydrochloride salt in powder on the streets. The chemical structures of PCP, ketamine, and MXE are present in Figure 5.
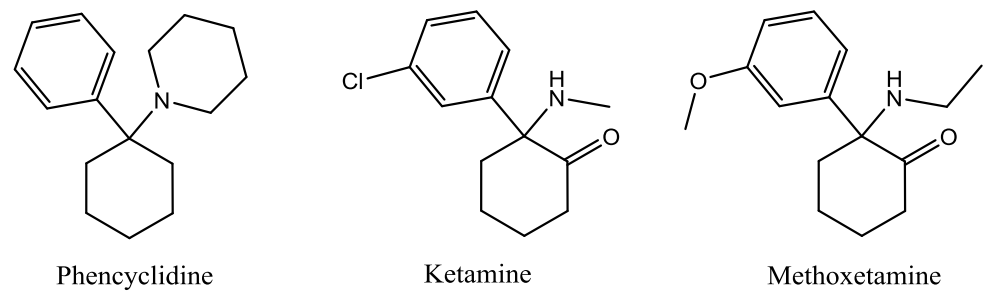

Figure 5. Chemical structures of phencyclidine, ketamine, and methoxetamine.

The frequent routes of administration for MXE are nasal insufflation and oral consumption, but other routes have also been reported including intramuscular and intravenous injections, sublingual, and rectal administration [55]. Although MXE is known as a 'legal' and 'bladder-friendly' alternative to ketamine, recent research revealed that significant bladder inflammation with subsequent fibrosis and renal toxicity is a potential risk [53]. Similar to ketamine, MXE is known as a glutamate N-methyl-Daspartate (NMDA) receptor antagonist and inhibits dopamine reuptake with a sub-micro molar affinity for the NMDA receptor and serotonin transporter, respectively $[53,56]$. Because of the resemblance of MXE to ketamine and PCP in their chemical structures and perceived effects on the user, the prevalence of MXE may affect public health significantly.

\subsubsection{Tryptamines}

Tryptamines are synthetic hallucinogenic indolealkylamines that are structurally similar to psilocybin, psilocin, and bufotenine [51]. Some examples of tryptamines include $N, N$-alpha-methyltryptamine (AMT), 5-methoxy- $N, N$-alpha-methyltryptamine (5MeO-AMT), N,N-dipropyltryptamine (DPT), N,N-dimethyltryptamine (DMT), 5- 
methoxy-N,N-diisopropyltryptamine $\quad(5-\mathrm{MeO}-\mathrm{DIPT}), \quad$ and $\quad 5$-methoxy- $N, N \quad$ dimethyltryptamine (5-MeO-DMT) as some them are presented in Figure 6 [51].

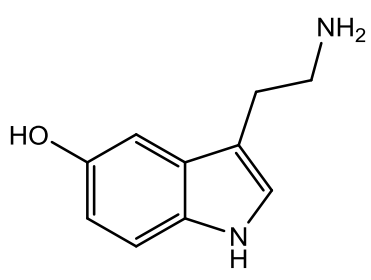

5-HT

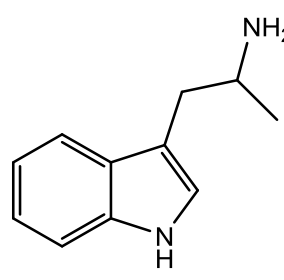

AMT

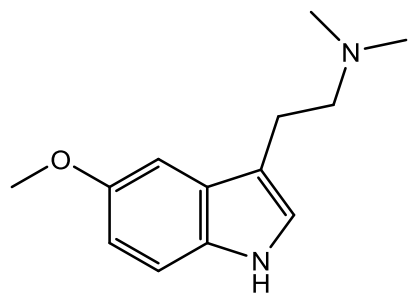

5-MeO-DMT

Figure 6. Chemical structures of 5-HT, AMT, and 5-MeO-DMT.

Listed as Schedule I drugs under the Controlled Substances Act, psilocybin (4phosphoryloxy- $N, N$-dimethyltryptamine) and its metabolite psilocin (4-OH $N, N$ dimethyltryptamine) are found in Psilocybe mushrooms [57]. Psilocybe mushrooms are also called 'magic mushrooms' because of their hallucinogenic properties [51]. Bufotenine (5-OH dimethyltryptamine) is also a naturally occurring tryptamine that is found in certain toads and Anadenanthera plants [51]. Among many others, psilocybin, DMT, 5-MeO-DMT, and 5-methodxy- $N, N$-diallyltryptamines (5-MeO-DALT) are the most commonly found tryptamines that represent a classical psychedelic profile by acting as agonists at the 5- $\mathrm{HT}_{2 \mathrm{~A}}$ and $5-\mathrm{HT}_{1 \mathrm{~A}}$ receptor subsequently inducing an increase in serotonin $[51,58]$. Depending on the routes of administration, tryptamines may show different activity; for instance, 5-MeO-DIPT, AMT and DPT are active when ingested whereas DMT and 5-MeO-DMT are effective when smoked or insufflated [51].

\subsubsection{Piperazines}

The final group of NPS covered in this research are piperazines. The original use of these compounds was to treat helminthic diseases [51]. There are two types of piperazine 
derivatives that are commonly abused, the benzylpiperazines and phenylpiperazines [51]. These include 1-benzylpiperazine (BZP), 1-methyl-4-benzylpiperazine (MBZP), $\mathrm{N}$-(3methylbenzyl)piperazine (MEBP), 1-(3-chlorophenyl)piperazine (mCPP), 1-(2-methoxyphenyl)piperazine, 1-(3-trifluoromethylphenyl)piperazine (TFMPP), and 1-methyl-3phenylpiperzaine (MeP) [59]. Figure 7 shows the chemical structures of BZP and TFMPP, which are among the most popular piperazine derivatives, known as 'party pills' or 'legal Ecstasy'. 1-Benzylpiperazine is listed as a Schedule I controlled substance under the Controlled Substance Act [57]. These derivatives are sold as pills or powders in a mixture of two to four compounds, for example, piperazines with MDMA or BZP and TFMPP with a ratio of 2 to 1 [51].

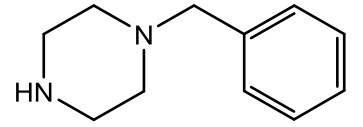

BZP

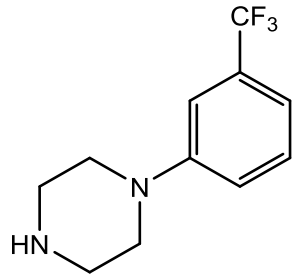

TFMPP

Figure 7. Chemical structures of BZP and TFMPP.

The mechanisms of action for BZP and TFMPP are known to be similar to MDMA, increasing both dopamine and serotonin levels [51]. 1-(3-Trifluoromethylphenyl) piperazine acts as a partial agonist/antagonist at 5- $\mathrm{HT}_{2 \mathrm{~A}}$ receptors and a full agonist at other 5-HT receptors, resulting in the increase of serotonin release. On the other hand, levels of dopamine are primarily increased by BZP. It is also known that BZP with TFMPP produces a synergistic effect together in a manner similar to MDMA. 


\subsection{Forensic Analysis of Controlled Substances}

The scientific working group for the analysis of seized drugs (SWGDRUG) provides a guideline for the forensic identification of seized drugs and requires the utilization of multiple uncorrelated techniques for the identification of controlled substances [60]. Table 1 shows different analytical techniques that can be used for the analysis of drug samples classifying into three categories on the basis of the discriminatory power of the technique. According to the SWGDRUG guidelines, the use of Category A technique requires at least one other technique from any category [60]. However, in the absence of a technique in Category A, at least three different techniques, two uncorrelated, are required for the identification of a controlled substance.

Table 1. Categories of analytical techniques suggested by SWGDRUG for the identification of controlled substances; adapted from [60].

\begin{tabular}{|c|c|c|}
\hline Category A & Category B & Category C \\
\hline Infrared Spectroscopy & Capillary Electrophoresis & Color Tests \\
\hline Mass Spectrometry & Gas Chromatography & Fluorescence Spectroscopy \\
\hline $\begin{array}{c}\text { Nuclear Magnetic Resonance } \\
\text { Spectroscopy }\end{array}$ & Ion Mobility Spectrometry & Melting Point \\
\hline Raman Spectroscopy & Liquid Chromatography \\
\hline X-ray Diffractometry & Microcrystalline Tests & Ultraviolet Spectroscopy \\
\hline & Pharmaceutical Identifiers & \\
\hline & Thin Layer Chromatography & \\
\hline & $\begin{array}{c}\text { Cannabis only: } \\
\text { Macroscopic Examination }\end{array}$ & \\
\hline
\end{tabular}


In forensic laboratories, the analysis of controlled substances is performed depending on the physical appearance of seized samples after the basic measurement of weight, volume, and/or count are taken [61-62]. For example, presumptive tests for general unknowns, substances in powder form, and illicit tablets are assessed by color tests, thin layer chromatography (TLC), microcrystalline tests, and/or gas chromatography (GC). Recently, DART-MS has been utilized as a screening tool for the analysis of controlled substance in the standard operation procedure of the Virginia Department of Forensic Science [63]. Confirmative tests to identify the presence of suspected compounds are then conducted using ultraviolet (UV) spectroscopy, Fourier transform infrared (FTIR) spectroscopy, and/or GC-MS depending on the availability of instrumentation and the standardized procedure from each laboratory. For plant material, such as marijuana products, examination under the microscope for morphological characteristics is conducted as part of the presumptive tests prior to TLC or color testing (Duquenois Levine test) [62].

\subsection{Principles of Instrumentation}

\subsubsection{Gas Chromatography}

As a universal and versatile separation technique, chromatography is applicable in various areas of chemistry and biochemistry, biology, quality control, research, and analysis [64]. The mechanism of separation is dependent on the distribution of the analytes between two phases, a stationary and a mobile phase. The relative affinity of analytes, which is determined by molecular structures and intermolecular forces, affect the distribution or partition of compounds between the stationary and mobile phases. 
Chromatography can be divided into three sub-groups depending on the type of mobile phase, including gas, liquid, and supercritical fluid [64].

Since the introduction of gas chromatography (GC) by James and Martin in 1952, it has been widely used for the separation of components in a mixture [65]. Gas chromatography is mainly composed of an injection port, column oven where the column stationary phase sits, carrier gas, and detector as illustrated Figure 8.

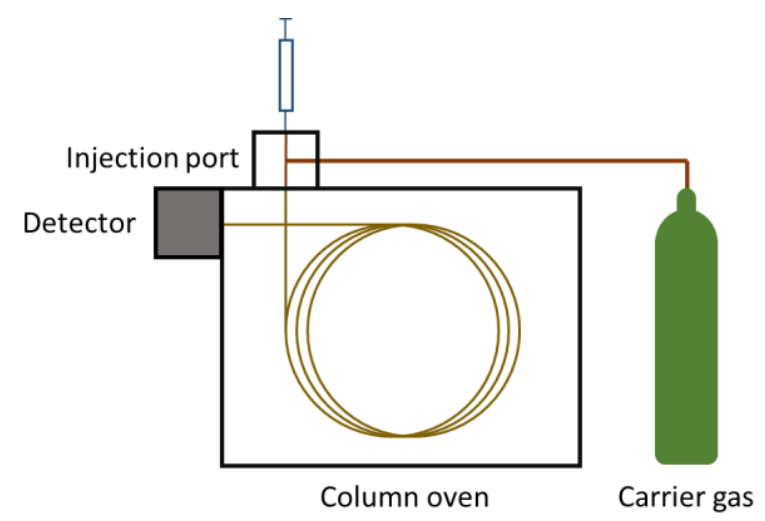

Figure 8. Schematic of gas chromatography.

A sample introduced into the injection port is volatilized and travels through a column (stationary phase) with a flow of a carrier gas (mobile phase) [64]. It is important that samples introduced into the GC are thermally stable and will not decompose during vaporization or separation in the high temperature column. The carrier gas has to be inert to prevent adsorption onto the column stationary phase during the transportation of gasphase analytes. In general, helium is commonly used as a carrier gas other than hydrogen in a capillary column because of a higher flow capability and safety considerations [66]. As a stationary phase, different types of columns can be utilized including coated and packed columns. A coated column, also called a capillary column, has a thin layer of a 
nonvolatile chemical coated onto the walls of the column, whereas a packed column is filled with an inert solid coated with the thin layer of the chemical [64]. Analytes carried into the column will be differentially retained depending on their affinity to the stationary phase and the component that is strongly retarded will be eluted later with the increased temperature of the column oven. Retention time refers to the time that an analyte spends in the column and is defined as:

$$
t_{r}^{\prime}=t_{r}-t_{m}
$$

where actual retention time $\left(t_{\mathrm{r}}^{\prime}\right)$ is the difference between the total time of a compound required to move through the column to the detector $\left(t_{r}\right)$, and the void volume, the time needed to replace the space by mobile phase to the analyte $\left(t_{\mathrm{m}}\right)$ [64]. Different types of detectors that are available for GC include, but are not limited to, the flame ionization detector (FID), thermal conductivity detector (TCD), thermionic specific detector (TSD), flame photometric detector (FPD), and electron capture detector (ECD) [65]. Gas chromatography is commonly coupled to a mass spectrometer as a powerful analytical technique providing both the separation capability by GC and the structural identification by the mass spectrometer (MS) [64]. As a hyphenated instrumentation, GC-MS is a widely used analytical technique because it is powerful, reliable and user friendly [5].

\subsubsection{Ion Mobility Spectrometry}

Ion mobility spectrometry (IMS) is an analytical technique that characterizes chemical compounds depending on the velocity of gas-phase ions under the influence of electrical field and a drift gas [67]. An ion swarm is produced by the ionization of transferred gaseous analytes into the ion source as shown in Figure 9. The ion swarm is 
then introduced into the drift region by a pulsed ion shutter and subsequently travels the drift region at different velocities. The velocity of the swarm is obtained through the electric field $(E)$ and varies depending on the size and shape of the ion. Therefore, smaller ions will move faster than larger ones through the electric field because of their collisional cross-section. Collision of ions with a faraday plate will generate a current flow of 10 to $1000 \mathrm{pA}$, which is amplified and transformed into a voltage signal. As a result, an ion mobility spectrum is plotted in the basis of the time taken by an ion swarm to move the distance $(d)$ in the drift region, called drift time $\left(t_{d}\right)$, and the response from detector (Figure 9).

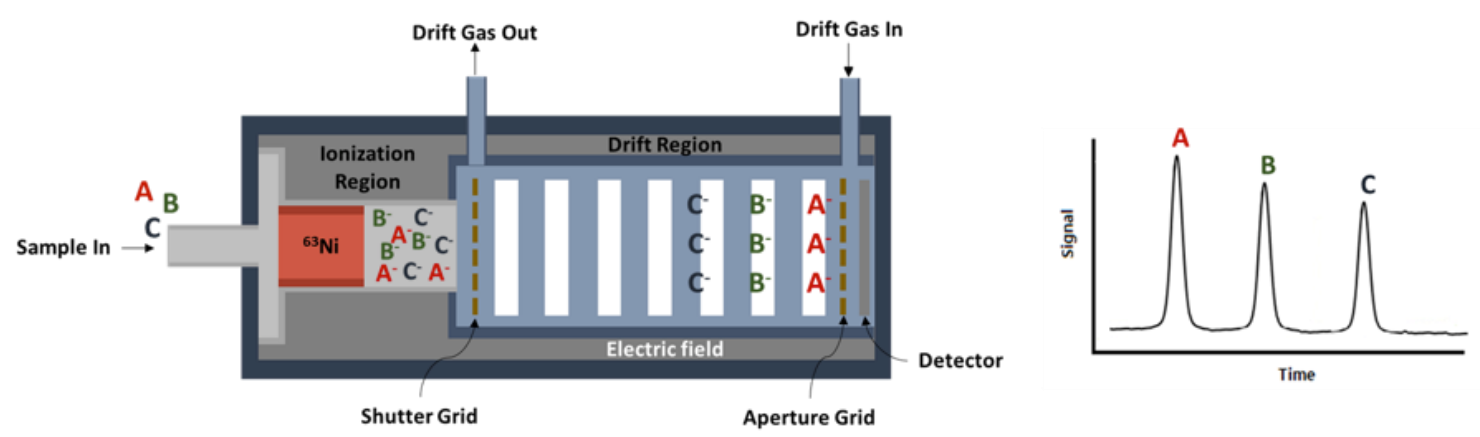

Figure 9. Schematic of a commercial IMS instrument with a ${ }^{63} \mathrm{Ni}$ radioactive ionization source and an example of ion mobility spectrum; Adapted and reproduced from [68].

The obtained velocity of the ion swarm under the electric field $(E)$ is called the drift velocity $\left(v_{d}, \mathrm{~cm} / \mathrm{s}\right)$, which is proportional to the electric field strength $(E, V / \mathrm{cm})$. The proportional constant between $v_{d}$ and $E$ is called the mobility coefficient $\left(K, \mathrm{~cm}^{2} / \mathrm{Vs}\right)$ as shown in Equation 2.

$$
K=\frac{v_{d}}{E}=\frac{d / t_{d}}{E}
$$


In order to standardize the mobility coefficient among various instruments and conditions, the mobility of ions is reported as reduced mobility $\left(K_{0}\right)$, which normalizes both temperature in Kelvin $(T)$ and pressure in torr $(P)$, as shown in Equation 3.[69]

$$
K_{0}=\frac{d}{t_{d} E}\left(\frac{273}{T}\right)\left(\frac{P}{760}\right)
$$

However, the accurate measurement of ion mobilities are often challenged by uncertainties derived from the accurate distance between the ion shutter and detector, the pressure and temperature inside of the drift tube, and the drift gas composition [70]. In order to compensate uncertainties that may arise from variations in different instruments, the reduced mobility of an unknown compound $\left(K_{0} u k n\right)$ can be determined by the ratio of the drift time of reference standard $\left(t_{d} r e f\right)$ and the drift time of unknown compound $\left(t_{d u k n}\right)$ as shown in Equation 4, where $K_{0}$ ref is the reduced mobility of reference standard [70].

$$
K_{0_{u k n}}=K_{0_{r e f}} \times \frac{t_{d_{r e f}}}{t_{d_{u k n}}}
$$

In IMS, the number of collisions between ions and drift gas molecules significantly affect the drift time of ions [69]. Therefore, pressure inside the drift tube considerably influences the mobility of ions because of the proportionality between numbers of collisions and the gas density (pressure). In addition, the drift of the ion swarm is affected by the energy from the electric field, the kind and temperature of the drift gas, the masses of the ions, and collision cross sections of the ions. The relationship of ion mobility with these parameters is presented as follows:

$$
K=\left(\frac{3 q}{16 N}\right)\left(\frac{2 \pi}{\mu k T}\right)^{\frac{1}{2}}\left(\frac{1+\alpha}{\Omega_{D}}\right)
$$


where $q$ is the charge of the ion and $N$ is the density of drift gas molecules, $\mu$ is the reduced mass of the ion $(m)$ and drift gas molecules $(M)$ expressed as $(m * M) /(m+M), k$ is the Boltzmann constant, $T$ is the temperature of the drift gas in Kelvin, $\alpha$ is the correction factor $\left(\alpha<0.02\right.$ for $m>M$ ), and $\Omega_{D}$ is the average ionic collision cross section which includes the electronic factors and structural parameters (size and shape) of the ion [69].

Ion mobility spectrometry has been widely used as a screening device in thousands of airports nationwide, offering several advantages over other analytical techniques including a relatively small size and light weight, in addition to an ambient pressure operation that allows for on-site use [67]. Furthermore, IMS provides fast response, high sensitivity, and ease of operation for high throughput analysis. Since the introduction of IMS in the analytical field for drug analysis by Karasek et al.[71-72], the popularity of IMS has increased over the years resulting in it being considered one of the fundamental techniques for the detection of illicit drugs. Successful detection of conventional designer drugs, such as MDMA and MDEA, extracted from human hair, as well as the detection of cocaine, MDMA, and marijuana by solid phase microextraction (SPME) were reported using a commercial IMS using ${ }^{63} \mathrm{Ni}$ as an ion source [73-74]. However, the limitation of IMS is the depletion of reactant ions along with an increased concentration of analyte molecules during the ionization process [67]. The ionization in positive mode involves the reaction between the analyte molecules and limited amount of reactant ions. The depletion of reactant ions results in a non-linear response of IMS with increasing concentration of the analyte. It may also result in the ambiguous analysis of 
complex seized drug samples because of the ionization competition between compounds in the sample.

\subsubsection{Radioactive ${ }^{63} \mathrm{Ni}$ ionization source}

As the most commonly found ion source in commercial IMS, the radioactive ${ }^{63} \mathrm{Ni}$ ionization source provides stable production of reactant ions, portability with low weight and power requirements, simplicity of use, and no maintenance or replacement of parts [69]. In this ion source, high energy electrons are emitted from the radioactive foils of 10 milliCurrie ${ }^{63} \mathrm{Ni}$ with a mean energy of $17 \mathrm{KeV}$. Emitted electrons then collide with the surrounding air or nitrogen producing the positive reactant ions. Produced by reactant ions, water clusters of a gas phase proton $\left(\mathrm{H}^{+}\left(\mathrm{H}_{2} \mathrm{O}\right)_{\mathrm{n}}\right)$, will react with molecules (M) from a sample and produce an adduct ion $\left(\mathrm{MH}^{+}\left(\mathrm{H}_{2} \mathrm{O}\right)_{\mathrm{n}}\right)$, which may be stabilized by the loss of water and terminates in the formation of product ions as follows [69]:

$$
M+\mathrm{H}^{+}\left(\mathrm{H}_{2} \mathrm{O}\right)_{n} \rightarrow \mathrm{MH}^{+}\left(\mathrm{H}_{2} \mathrm{O}\right)_{n-\mathrm{x}}+\chi_{2} \mathrm{O}
$$

When the proton affinity of the analyte molecule in gas-phase is greater than that of water $(691 \mathrm{~kJ} / \mathrm{mol})$, this reaction will become favorable. In the analysis of compounds using IMS, the reliable measurement of samples is required without the distortion of chemical information [67]. The consistent measurement can be achieved by adapting online calibration of the analyzer and utilizing reagent gases, which may reduce chemical interference from the sample matrix and improve the response resulted from more specific reactions between compounds with different proton affinity. For drug detection in the positive mode, nicotinamide (NA) is commonly used as both a calibrant and a reagent gas [75]. The drift gas containing trace amounts of nicotinamide (NA) flows 
constantly into the drift tube and ionization/reaction chamber and the dominant ionized species, (NA) $\mathrm{H}^{+}$, are then produced in the stand-by mode. When a sample is introduced, it is ionized by proton transfer from protonated NA to the sample molecule (M) in the analyze mode as shown in Equation 7 [75].

$$
M+(N A) H^{+} \rightarrow M H^{+}+N A
$$

The ionization process in this IMS is preferred only when the proton affinity of $M$ is greater than that of NA. Therefore, compounds that are favorable in this ionization process can be detected as protonated ions $\left(\mathrm{MH}^{+}\right)$under this IMS conditions [75]. Although the ${ }^{63} \mathrm{Ni}$ ion source is widely used in many commercial IMS, there are some disadvantages including special permit requirements for handling, the formation of nickel oxides or salts that may be released, and cost of proper disposal [69].

\subsubsection{Coupling of Electrospray Ionization and Mass Spectrometry with Ion Mobility Spectrometry}

Traditional IMS operates with a radioactive ionization source $\left({ }^{63} \mathrm{Ni}\right)$ and has been utilized for more than 30 years as a method for the detection of vapor-phase explosives, illicit drugs, and chemical warfare agents [73-74, 76-77]. However, there are several limitations associated with this conventional IMS which includes the inability to analyze nonvolatile compounds and high false positive rates because of low resolving power [76]. The interface of IMS with an ESI source enables the introduction and detection of nonvolatile compounds and the direct analysis of aqueous samples [34, 78]. In addition, the implementation of ESI source expands the application of IMS to other fields including 
biomolecule analysis [79] and it also eliminates handling limitations resulting from the cumbersome restrictions of the radioactive source [32].

The coupling of IMS with a mass spectrometer (MS) was first developed back in the early 1960s allowing for the investigation of analytes in a two-dimensional approach, the mobility and mass-to-charge ratio $(\mathrm{m} / \mathrm{z})$ of ions in the gas-phase [80]. When IMS is in coupled to MS, it significantly reduces the possibility of false positive results with the complementary spectral data obtained from a MS on the basis of the size and mass of ions $[76,81]$. While the coupling of atmospheric pressure IMS and mass spectrometers is well described in a report by the Sandia National Laboratories, a proposed schematic of the interface between two instruments is shown in Figure 10 [82]. The interface of IMS and MS can be challenging because both instruments are destructive techniques, meaning that the ions are destroyed at the moment of measurement. A solution to this issue has been proposed that a portion of ions is introduced to the MS passing through a small hole created on the Faraday plate in the IMS [82].

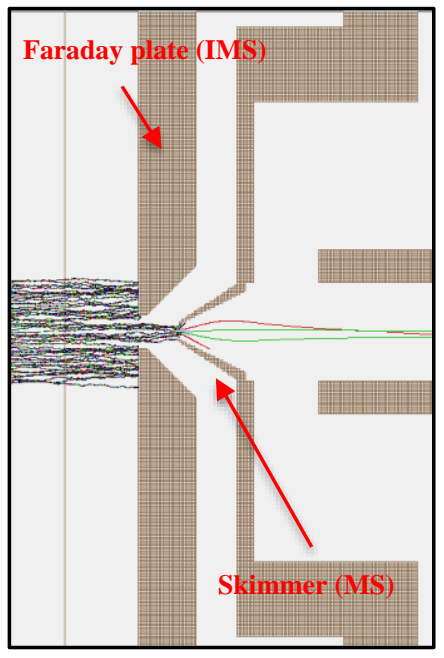

Figure 10. Proposed schematic of the interface close-up between an ion mobility spectrometer and a mass spectrometer; adapted from [82]. 
There are many different types of mass spectrometers that can be coupled to IMS, including time-of-flight (TOF), linear quadrupoles, trapping devices, Fourier transform ion cyclotron resonance (FTICR), and magnetic sector spectrometers [80]. It is also possible to interface these mass analyzers with IMS in two configurations; positioning the mass analyzer before the drift tube or the other way around [80]. In the following section, different types of mass spectrometers that have been utilized in this dissertation will be explained in detail.

\subsubsection{Mass Spectrometry}

Mass spectrometry (MS) is by far the most applicable analytical technique that can provide information such as (1) the elemental composition of a sample, (2) the structure of organic, inorganic, and biological molecules, (3) the qualitative and quantitative composition of samples in complex mixtures, (4) the structure and composition of a solid surface, and (5) the isotopic ratios of atoms in samples [83]. Generally, a mass spectrometer is composed of an inlet, ion source, mass analyzer, and detector, which are housed under vacuum. A micro amount of sample is introduced into the mass spectrometer through the inlet system where it is ionized by applying thermal or electrical energy in the ion source. A stream of positive or negative ions in gas-phase are then accelerated and separated according to the mass-to-charge ratio $(\mathrm{m} / \mathrm{z})$ in the mass analyzer under a high vacuum. A detector converts the stream of ions to an electrical signal that can be displayed on a computer [84]. 


\subsubsection{Ion Sources}

Ion sources play an important role for the formation of gaseous analyte ions as well as the scope and the utility of a mass spectrometer [83]. Ionization techniques can be categorized into two groups, gas-phase and desorption sources. In gas-phase sources, such as electron ionization (EI), chemical ionization (CI), and field ionization (FI), the compounds are first vaporized and then ionized; therefore, these sources are limited to analytes that are volatile and thermally stable [83]. On the other hand, desorption sources convert solid or liquid sample into gaseous ions directly, facilitating the ionization of nonvolatile and thermally labile compounds. Desorption sources include field desorption (FD), electrospray ionization (ESI), matrix-assisted laser desorption ionization (MALDI), plasma desorption (PD), fast atom bombardment (FAB), secondary-ion mass spectrometry (SIMS), and thermospray ionization (TS) [83]. Advance ionization techniques have also been introduced in the last few years allowing for the ionization of samples at atmospheric pressure and at ground potential. These ambient ionization techniques include, but are not limited to, desorption electrospray ionization (DESI) developed by Cooks and his co-workers and direct analysis in real time (DART) developed by Cody et al. [85]. The recent development of ambient ionization techniques, DESI and DART, facilitated the direct MS analysis of samples in both qualitative and quantitative investigations with minimal to no sample preparation in most cases [85]. The detailed description of ionization techniques that are implemented in this research will be discussed in the following section and the DART ion source is described in section 2.3.4. 


\section{Electron Ionization}

As one of the most common ion sources in organic mass spectrometry, the EI source serves well to produce gaseous ions from many organic molecules [84]. The ionization occurs when the gaseous compounds are collided with the accelerated electrons that are emitted from a heated filament as shown in Figure 11. The ionization process in the EI source is favorable when an energy transfer that leads to various electronic excitations may occur in the filament or when samples containing a high vapor pressure are introduced directly into the source [84].

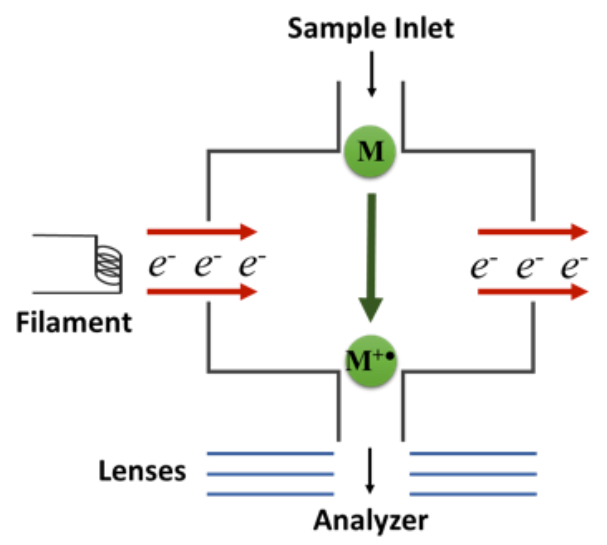

Figure 11. Diagram of an electron ionization source; adapted and reproduced from [84].

Although most organic molecules are ionized well at electron energies between 10 and 20 $\mathrm{eV}$, the EI source is generally operated at $70 \mathrm{eV}$ to minimize the variation in the observed number of ions produced; a parameter that may affect the pattern of the produced spectrum. However, the excessive energy from the EI source often results in the extensive fragmentation of compounds [84]. Consequently, the molecular ions are not 
always present in a spectrum. Nevertheless, this extensive fragmentation can be useful in providing structural information for the analysis of unknown samples.

\section{Chemical Ionization}

The chemical ionization (CI) source has the advantage of producing a spectrum with less fragmentations than that observed for EI and is therefore referred to as a soft ionization technique [83-84]. One main difference between the EI and CI sources is the use of a reagent gas for the CI source as illustrated in Figure 12. Electrons given off from the filament will preferentially ionize reagent gas molecules and the produced ions will collide with other excess reagent gas molecules establishing an ionization plasma [84]. Methane $\left(\mathrm{CH}_{4}\right)$ is one of the most commonly used reagent gases although other reagent gases, such as isobutane and ammonia, are readily utilized in the CI source.

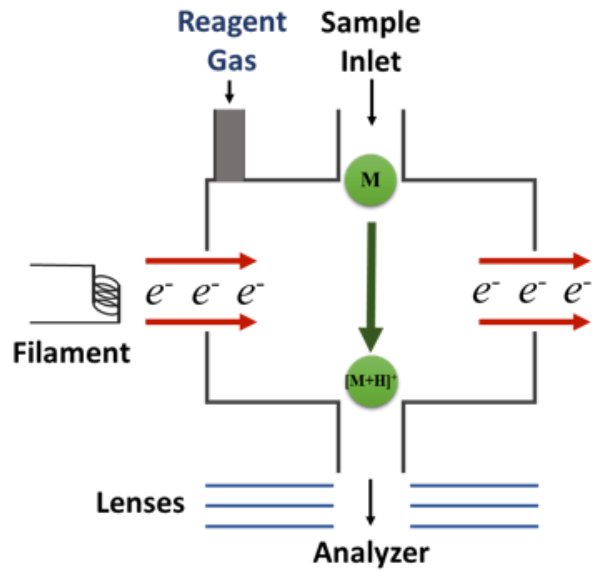

Figure 12. Diagram of a chemical ionization source.

As a reagent gas, methane, is reacted with the electrons ejected from the filament yielding the methane ions (Equation 8) [84]. The majority of methane ions will collide with other 
methane molecules producing the methanium ion, $\mathrm{CH}_{5}{ }^{+}$(Equation 9). These methanium ions will then subsequently ionize the analyte by proton transfer (Equation 10).

$$
\begin{gathered}
\mathrm{CH}_{4}+\mathrm{e}^{-} \rightarrow \mathrm{CH}_{4}^{+\bullet}+2 \mathrm{e}^{-} \\
\mathrm{CH}_{4}^{+\bullet}+\mathrm{CH}_{4} \rightarrow \mathrm{CH}_{5}^{+}+\mathrm{CH}_{3}^{\bullet} \\
\mathrm{M}+\mathrm{CH}_{5}^{+} \rightarrow[\mathrm{M}+\mathrm{H}]^{+}+\mathrm{CH}_{4}
\end{gathered}
$$

However, a small number of methane ions will be dissociated into the methyl cation and collide with other methane molecules yielding ethenium, $\mathrm{C}_{2} \mathrm{H}_{5}{ }^{+}$(Equation 11 and 12) [84]. This positive ion will produce the ethyl adduct by reacting with the analyte (Equation 13).

$$
\begin{gathered}
\mathrm{CH}_{4}^{+\bullet} \rightarrow \mathrm{CH}_{3}^{+}+\mathrm{H}^{\bullet} \\
\mathrm{CH}_{3}^{+}+\mathrm{CH}_{4} \rightarrow \mathrm{C}_{2} \mathrm{H}_{5}^{+}+\mathrm{H}_{2} \\
\mathrm{M}+\mathrm{C}_{2} \mathrm{H}_{5}^{+} \rightarrow\left[\mathrm{M}+\mathrm{C}_{2} \mathrm{H}_{5}\right]^{+}
\end{gathered}
$$

Other reactions also occur from the $\mathrm{CH}_{4}{ }^{+}$fragment, $\mathrm{CH}_{2}{ }^{+}$(Equation 14), resulting in the formation of an adduct, $\left[\mathrm{M}+\mathrm{C}_{3} \mathrm{H}_{5}\right]^{+}$, through the reaction from Equation 15 to 17.[84]

$$
\begin{gathered}
\mathrm{CH}_{4}^{+\bullet} \rightarrow \mathrm{CH}_{2}^{+\bullet}+\mathrm{H}_{2} \\
\mathrm{CH}_{2}^{+\bullet}+\mathrm{CH}_{4} \rightarrow \mathrm{C}_{2} \mathrm{H}_{3}^{+}+\mathrm{H}_{2}+\mathrm{H}^{\bullet} \\
\mathrm{C}_{2} \mathrm{H}_{3}^{+}+\mathrm{CH}_{4} \rightarrow \mathrm{C}_{3} \mathrm{H}_{5}^{+}+\mathrm{H}_{2} \\
\mathrm{M}+\mathrm{C}_{3} \mathrm{H}_{5}^{+} \rightarrow\left[\mathrm{M}+\mathrm{C}_{3} \mathrm{H}_{5}\right]^{+}
\end{gathered}
$$




\section{Electrospray Ionization}

The electrospray ionization (ESI) source was originally developed to aid the ionization of large organic molecules, such as proteins, that are not easily converted to the gas-phase by the traditional ion sources [86]. Recently, ESI has been widely used not only for large biomolecules analysis, but also for small polar molecules, utilizing the soft ionization capability of the ESI [84]. In the ionization process of ESI source, a liquid sample passes through a capillary tube at a low flow rate, generally between 1 to 10 $\mu \mathrm{L} / \mathrm{min}$ [84]. The applied potential difference $(3-6 \mathrm{kV})$ produces a strong electric field between the capillary tip and the counter-electrode in the distance of $0.3-2 \mathrm{~cm}$ as shown in Figure 13. A charge accumulated under this strong electric field in atmospheric pressure will overcome the surface tension from the liquid to form highly charged small droplets at the end of the capillary [84].

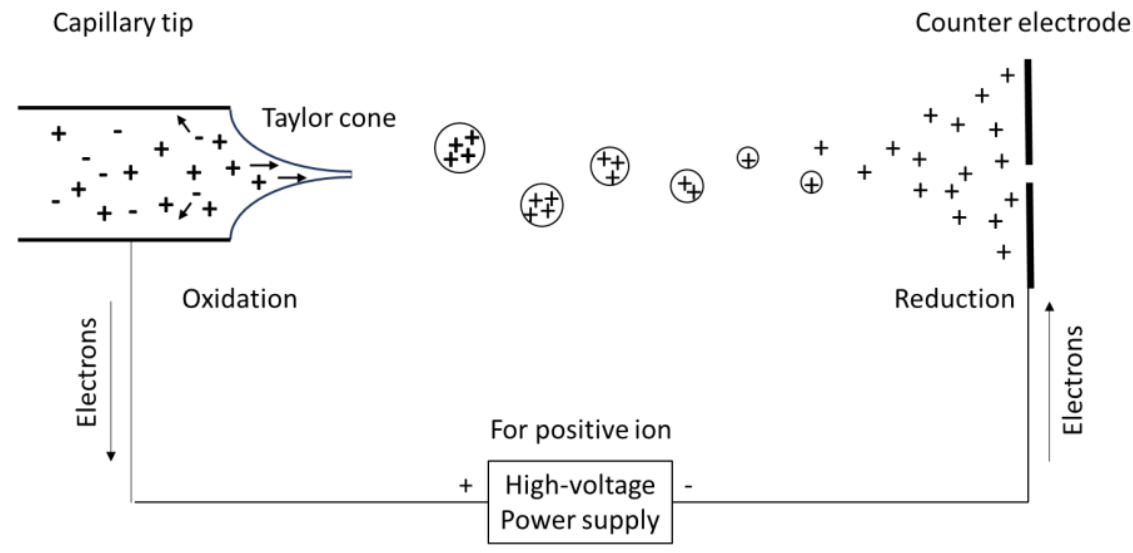

Figure 13. Formation of a Taylor cone in the process of positive ion production in the electrospray ionization; reproduced and modified from [84].

As illustrated in Figure 13, a drop at the capillary tip elongates under the strong electric field producing a Taylor cone [84]. The elongation of the droplet results from the 
evaporation of solvent in the droplet [86]. As a result, the charge density on the surface of the droplet increases reaching the Rayleigh limit at which the surface tension and Coulomb repulsion become the same order [86]. A series of smaller droplets are also produced from this Taylor cone and undergo a similar process of solvent evaporation and charge accumulation. While the extent of ionization is limited compared to EI and CI, the ESI source is advantageous as it can produce multiply charged ions from large molecules while preserving molecular ions.

\subsubsection{Mass Analyzers}

Gas-phase ions that are produced by different ionization sources are separated according to their mass-to-charge ratio $(\mathrm{m} / \mathrm{z})$ in a mass analyzer [84]. Although all mass analyzers utilize static or dynamic electric and magnetic fields, in isolation or combination, different principles are applied for the separation of ions. For example, separation of ions in a quadrupole occurs on the basis of $\mathrm{m} / \mathrm{z}$ which determines the velocity of ions influenced by the trajectory stability and time-of-flight of ions. Mass analyzers can be categorized into two groups, pulsed and continuous, in terms of ion sampling. In continuous sampling mass analyzers, such as magnetic sector and quadrupole analyzers, ions of different masses are transmitted along a time scale. These are also known as space-based analyzers, which only allow the specific ion to pass through a flight tube at a measured time. On the other hand, in pulsed sampling analyzers, such as ion trap (IT), time-of-flight (TOF), ion cyclotron resonance (ICR), and orbitrap (OT), ions are simultaneously transmitted into the space where the separation occurs. Pulsed sampling analyzers are, therefore, often referred to as time-based analyzers. The 
detailed mechanism of separation for quadrupole, TOF, and tandem mass spectrometers will be covered in the following sections.

\section{Quadrupole}

Quadrupole mass analyzers are the most common space-based mass analyzer, composed of four rods aligned perfectly parallel to one another, thus providing a hyperbolic internal space (Figure 14) [84]. The quadrupole analyzer separates introduced ions on the basis of their $\mathrm{m} / \mathrm{z}$ utilizing the stability of the trajectories in oscillating electric fields. An opposite potential is applied to the parallel rods in the $\mathrm{x}$ - and y-axis, resulting in oscillation of the ions as they move towards the detector. The potential applied to the rods is represented as $\Phi_{0} ; U$ is the applied direct current (DC) voltage, $V$ is the applied radio frequency $(\mathrm{RF})$ voltage, and $\omega$ is the angular frequency, as shown in Equation 18. On the basis of this relationship, ions with specific $\mathrm{m} / \mathrm{z}$ can be selectively permitted to pass through the rods and reach to the detector by controlling $U$ and $V$ [84].
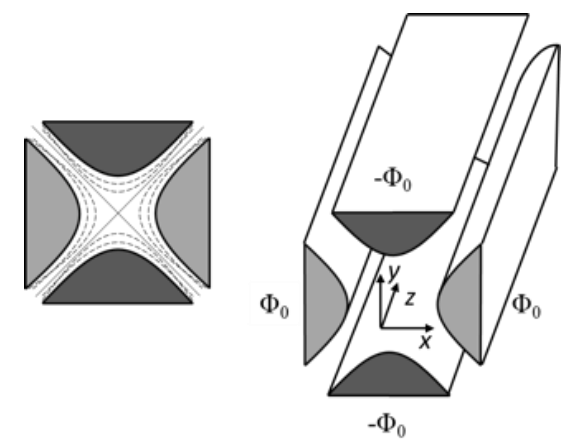

Figure 14. Diagram of a quadrupole mass analyzer with hyperbolic rods and applied potentials; reproduced and modified from [84].

$$
\Phi_{0}=U-V \cos \omega t
$$


Generally, the operation of the quadrupole analyzer is at single mass unit resolution; therefore, it is possible to separate two peaks that are only one mass unit apart. However, the highest mass range is limited to around $\mathrm{m} / \mathrm{z} 3000$ with this analyzer. Regardless, the unique characteristics of quadrupole mass analyzers, such as their compact size and relatively easy operation and maintenance, has led to the widespread use of quadrupole analyzer coupled to gas chromatography [87].

\section{Time-of-Flight}

In the TOF mass analyzer, ions are accelerated into a flight tube by an electric field. They are then separated according to their velocities as they drift through the flight tube, also called a free-drift region [84]. An electric potential energy of ions from the accelerating potential is converted into the kinetic energy of ions which determines the ions velocity. These accelerated ions then move straight through a free-drift region at a constant velocity. As shown in Equation 19, the time that ions take to travel through the flight tube is related to the $\mathrm{m} / \mathrm{z}$ of ions where $L$ is the distance and $V_{s}$ is the accelerating potential [84].

$$
t^{2}=\frac{m}{z}\left(\frac{L^{2}}{2 e V_{S}}\right)
$$

Since the distance and potential are constant for a given spectrometer, the $\mathrm{m} / \mathrm{z}$ can be determined by measuring the time taken to travel this flight tube. The advantages of TOF analyzers include a broad mass range, high sensitivity resulting from high transmission efficiency, and a fast analysis time [84]. However, peak broadening is a concern in TOF analyzers; a phenomenon that arises when ions with the same $m / z$, but different kinetic 
energies reach the detector at slightly different times. In order to reduce the kinetic energy dispersion of ions, a reflectron, also known as ion mirrors, is utilized in modern TOF mass analyzers [84]. Ions with more kinetic energy will move faster and penetrate a reflectron deeper than ions with less kinetic energy. As a result, the ions with slower velocity will reach to the detector at the same time as the faster ions with the same $\mathrm{m} / \mathrm{z}$, as faster ions will spend more time in the reflectron. The energy spread among ions can also be corrected by introducing a time delay between ion formation and extraction, which is known as a delayed pulsed extraction.

\section{Tandem Mass Spectrometry}

Tandem mass spectrometry (MS/MS) interfaces two stages of the mass analyzer thus providing high sensitivity for the selectively ionized target compound [87]. In general, a first analyzer serves as a mass filter that isolates a precursor ion (previously described as a parent ion) and a second analyzer separates the product ions (previously described as a daughter ion) [84]. It is also possible to have multiple steps in terms of the isolation and fragmentation of a precursor ion in some tandem mass spectrometers, labelled as $\mathrm{MS}^{n}$, such as IT and ICR [84]. There are four main scan modes available in tandem mass spectrometry, including product ion scan, precursor ion scan, neutral loss scan, and selected reaction monitoring (SRM) as illustrated in Figure 15 [84]. In the product ion scan mode, a precursor ion of interest is chosen and the product ions, also called fragment ions, are subsequently determined from collision-induced dissociation (CID). In contrast, in precursor ion scan mode, a product ion is selected and all the precursor ions that produce the targeted product ion are detected. The neutral scan mode 
is composed of the selection of a neutral fragment and the determination of all the fragmentation resulting from the loss of that neutral fragment. It is important to note that precursor ion scan and neutral loss scan modes are not available with time-based mass analyzers because these two scan modes require the second spectrometer to focus on a selected ion, or the simultaneous operation of both spectrometers. In the SRM mode, both analyzers are targeted on user-defined specific ions and selecting a fragmentation reaction rather than a fragment. The SRM mode provides high sensitivity and selectivity as the analyzer spend longer times focused on the precursor and fragmentation ions.

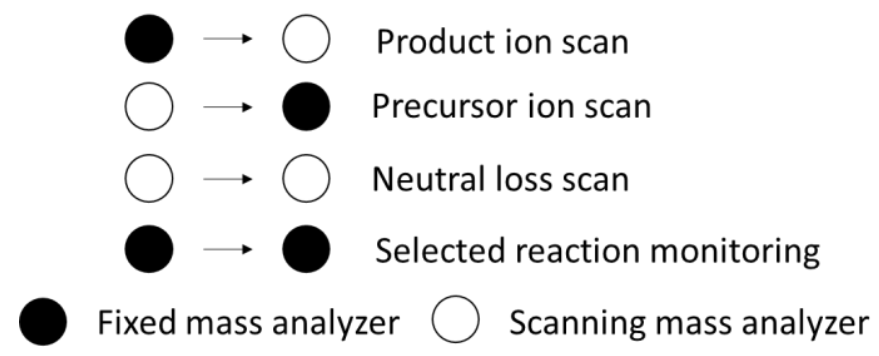

Figure 15. Symbolism for the representation of four different scan modes as proposed by Cooks et al.; adapted and modified from [84].

To facilitate the fragmentation of a selected precursor ion, a process called 'CID' occurs in a collision cell that is normally placed between the two mass analyzers [84]. During CID, collisions between the low- or high-energy accelerated ions and a static target (the collision gas) occur resulting in the transfer of the kinetic energy into internal energy. Hence, tandem mass spectrometry provides broad applications, including structural elucidation, determination of fragmentation mechanism and elementary compositions, and high selective and high sensitive analysis [84]. 
A triple quadrupole mass spectrometer $(\mathrm{QqQ})$ is one common type of tandem mass spectrometer, which utilizes three stages of quadrupoles as shown in Figure 16. The first and third quadrupole, Q1 and Q3, work as mass analyzers and a middle quadrupole (or hexapole) operates as a collision cell in RF-only mode [84]. As mentioned, the precursor ion is selected and introduced into the collision cell where the collision of the selected ion (precursor ion) occurs in order to provide the product ions for mass analysis in Q3.

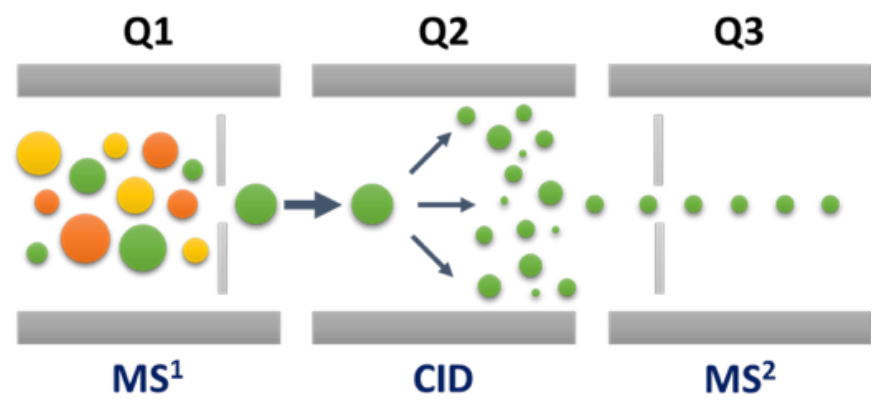

Figure 16. Diagram of a triple quadrupole mass analyzer.

A number of studies have been reported using a triple quadrupole mass spectrometer coupled to chromatographic techniques for the differentiation of drug isomers [20, 22], high sensitive analysis of drugs in hair [88-89], and analysis of emerging NPS [24, 27, 90].

A combination of quadrupole and time-of-flight mass analyzers is the most successful type of hybrid instruments as it shows powerful and robust performance providing high sensitivity and mass accuracy [84]. The quadrupole time-of-flight (QTOF) tandem mass spectrometer is normally described as a triple quadrupole with the replacement of an orthogonal accelerator TOF analyzer instead of the third quadrupole (Q3) as illustrated in Figure 17. In the general configuration of QTOF, there is an extra 
quadrupole $(\mathrm{Q} 0)$ that provides collisional damping; therefore, the system is composed of three quadrupoles, Q0, Q1, and Q2, followed by a TOF analyzer [91]. Although the Q0 and Q2 are equipped with hexapoles in commercial instruments, the basic principles remain similar to operating in RF-only mode.

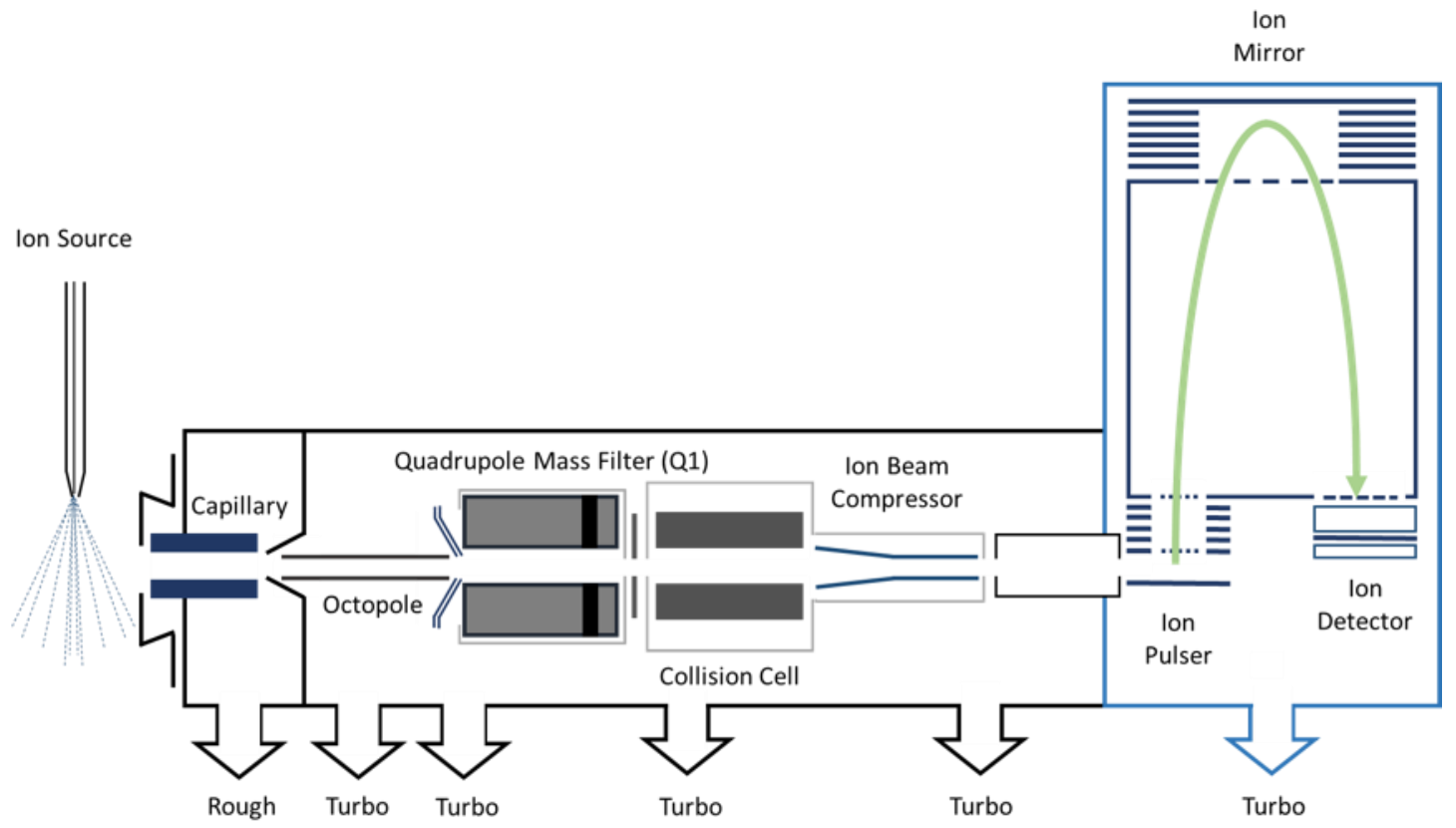

Figure 17. Schematic of a commercial high resolution quadrupole time-of-flight mass spectrometer; reproduced and modified from [92].

MS mode and MS/MS mode are both possible in a QTOF instrument [84]. In MS mode (full scan), the two quadrupoles, Q1 and Q2, act as ion guides in RF-only mode. In MS/MS mode (product ion scan), the Q1 works as the mass filter that transmits and selects only the precursor ion of interest. The selected ion is then accelerated to an energy of between 20 and $200 \mathrm{eV}$ before it is introduced into the collision cell (Q2) where CID occurs in the presence of neutral gas molecules (generally nitrogen or argon). The remaining precursor ions and resulting product ions from the collision cell are 
subsequently accelerated again to the required energy, normally several tens of electronvolts $(\mathrm{eV})$ per unit charge, and focused by ion optics (ion beam compressor) for introduction into the ion modulator (ion pulser) [91]. The process of ion selection in Q2 controls the kinetic energy of ions as well as focuses the ions by collision cooling for better sensitivity and resolution [84]. TOF mass separation occurs when a pulsed electric field is applied at the ion pulser, pushing ions into the flight tube, and finally the detector.

The QTOF mass spectrometer (QTOF-MS) is a highly versatile instrument, and, when coupled to liquid chromatography (LC) has been widely used for a range of applications. For example, QTOF-MS has been implemented in the analysis of pharmaceuticals and their metabolites in water samples for environmental chemistry applications [93-94]. It has also been used for the characterization of synthetic cathinones in forensic chemistry application [95-96].

\subsubsection{Direct Analysis in Real Time Mass Spectrometry}

Technically, direct analysis in real time (DART) is a relatively new ionization source for the rapid non-contact analysis of samples at ambient pressure and ground potential, which was first developed by Cody et al. [97]. Initially, the development of DART was motivated by the desire to replace the radioactive sources (nickel-63 and americium-241) that are required in chemical agent monitors and toxic industrial chemical sensors. The commercial DART ion source, IonSense DART ${ }^{\circledR}$-SVP (Saugus, MA, USA), is composed of a chamber with multiple stages of tubes, where a gas flows at atmospheric pressure. A schematic of the DART source is shown in Figure 18 [98]. A kilovolt potential applied between a needle electrode and a grounded counter electrode 
initiates a glow discharge at ambient pressure [99]. As the gas passes through these multiple tubes with an intermediate electrode, gas heater, and grid electrode at the exit of the DART source, the gas undergoes conversion to the metastable state. The ionization process takes place when these metastable species react with a gaseous, liquid, or solid sample in the open space between the DART source outlet and the mass spectrometer inlet [99].

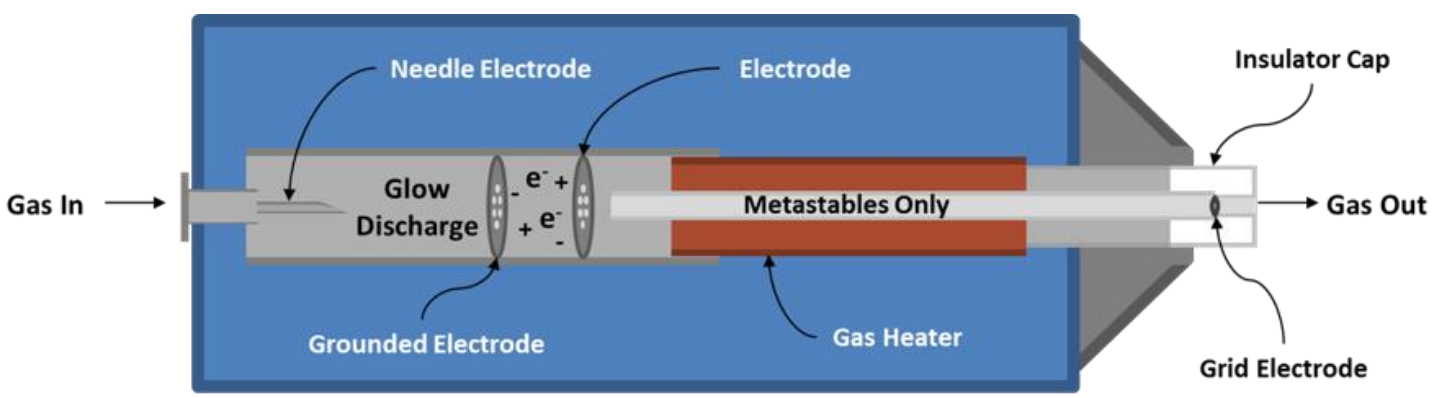

Figure 18. Schematic of the DART ionization source; reproduced and modified from [98].

The formation of positive ions in DART is governed by Penning ionization and proton transfer [99]. In Penning ionization, energy is transferred from a metastable atom or molecule $\left(\mathrm{N}^{*}\right)$ to an analyte molecule $(\mathrm{M})$, producing the molecular ion of the analyte $\left(\mathrm{M}^{+\bullet}\right)$ and an electron $\left(\mathrm{e}^{-}\right)$as shown in Equation 20. The transfer of energy is possible when the ionization energy of an analyte is lower than that of a metastable atom or molecule. It is known that the energy of helium atoms $(\mathrm{He})$ in its excited electronic state $\left(2^{3} \mathrm{~S}\right)$ is $19.8 \mathrm{eV}$ [99]. The energy of excited state He is greater than the energy of other long-lived noble gas atoms (Helium*>Neon*>Argon*>Krypton*) as well as any potential organic molecules [100]. The higher ionization energy of metastable helium atoms (He*) makes helium the most effective gas to use for DART. 


$$
\mathrm{N}^{*}+\mathrm{M} \rightarrow \mathrm{M}^{+\cdot}+\mathrm{e}^{-}
$$

In order for the formation of positive ions by proton transfer, atmospheric moisture is first ionized by $\mathrm{He}^{*}$ with extremely favored efficiency compared to other species present in atmosphere (Equation 21) [99]. The protonated molecule, $[\mathrm{M}+\mathrm{H}]^{+}$, is then produced when the analyte has a higher proton affinity than the water cluster ions, $\left[\left(\mathrm{H}_{2} \mathrm{O}\right)_{n}+\mathrm{H}\right]^{+}$ (Equation 22) [99].

$$
\begin{gathered}
\mathrm{He}\left(2^{3} \mathrm{~S}\right)+\mathrm{nH}_{2} \mathrm{O} \rightarrow\left[\left(\mathrm{H}_{2} \mathrm{O}\right)_{\mathrm{n}-1}+\mathrm{H}\right]^{+}+\mathrm{OH}^{\bullet}+\mathrm{He}\left(1^{1} \mathrm{~S}\right) \\
{\left[\left(\mathrm{H}_{2} \mathrm{O}\right)_{n}+\mathrm{H}\right]^{+}+\mathrm{M} \rightarrow[\mathrm{M}+\mathrm{H}]^{+}+\mathrm{nH}_{2} \mathrm{O}}
\end{gathered}
$$

Compounds with carbonyl functional groups, including acids, esters, ketones, and peroxides may also produce ammonium adducts $\left[\mathrm{M}+\mathrm{NH}_{4}\right]^{+}$in the presence of an ammonium source in surrounding environment [99].

In negative mode, thermal electrons are produced by Penning ionization of nitrogen $\left(\mathrm{N}_{2}\right)$ in which the energy is transferred from $\mathrm{He}^{*}$ to $\mathrm{N}_{2}$ (Equation 23) [100]. The thermal electrons are captured by atmospheric oxygen; consequently, the produced negatively charged oxygen $\left(\mathrm{O}_{2}^{-\bullet}\right)$ then serve as reagent ions that can form adducts with the analyte, M (Equation 24 and 25).

$$
\begin{gathered}
\mathrm{He}^{*}+\mathrm{N}_{2} \rightarrow \mathrm{He}+\mathrm{N}_{2}^{+\bullet}+\mathrm{e}^{-} \\
\mathrm{O}_{2}+\mathrm{e}^{-} \rightarrow \mathrm{O}_{2}^{-\bullet} \\
\mathrm{O}_{2}^{-\bullet}+\mathrm{M} \rightarrow\left[\mathrm{M}+\mathrm{O}_{2}\right]^{-\bullet}
\end{gathered}
$$

However, the exact ionization process in DART is still not well understood and other ionization mechanisms are also feasible [99]. Alternative ionization processes may 
include direct electron capture (Equation 26), dissociative electron capture (Equation 27), deprotonation by dissociation or reaction with a base (Equation 28), and anion attachment (Equation 29) [100].

$$
\begin{gathered}
\mathrm{M}+\mathrm{e}^{-} \rightarrow \mathrm{M}^{-\bullet} \\
\mathrm{MX}+\mathrm{e}^{-} \rightarrow \mathrm{M}^{-}+\mathrm{X}^{\bullet} \\
\mathrm{MH} \rightarrow[\mathrm{M}-\mathrm{H}]^{-}+\mathrm{H}^{+} \\
\mathrm{M}+\mathrm{X}^{-} \rightarrow[\mathrm{M}+\mathrm{X}]^{-}
\end{gathered}
$$

Since the introduction of DART in the early 2000s, the DART source coupled with a time-of-flight (TOF) mass spectrometer (often JEOL AccuTOF) has been widely used for many applications, including food quality and safety [101-102], pharmaceutical and clinical applications [103-104], environmental applications [105-108], and many forensic applications [109-111]. Howlett and Steiner have shown the potential use of DART-MS with thin layer chromatography (TLC) for the identification of drug compounds as an alternative to GC-MS with the separation capability [112]. The DART-MS approach has also been utilized for the rapid identification of some synthetic cannabinoids and cathinones by Dunham et al. [36], Musah et al. [30, 38], and Lesiak et al. [31, 113]. With regard to the detection capability of controlled substances, DART-MS has recently been adopted as a screening technique in a number of forensic laboratories across the country [62]. 


\section{CHAPTER 3. MATERIALS AND METHODS}

All reference standards of NPS listed in Appendix 1 were provided by Cayman Chemical (Ann Arbor, MI, USA), in powder or solution form. For those reference standards in powder form, standard stock solutions were prepared in different organic solvents to the following concentrations, 1000,2000 , and $2500 \mu \mathrm{g} / \mathrm{mL}$, according to the solubility of each compound. Methanol was primarily used to dissolve those standards, except some NPS standards that are prepared in dimethyl sulfoxide (DMSO) and ethanol (EtOH) as presented in Table 2.

Table 2. List of NPS standards that are prepared in dimethyl sulfoxide and ethanol.

\begin{tabular}{|c|c|c|c|}
\hline NPS & $\begin{array}{l}\text { Molecular } \\
\text { Formula }\end{array}$ & $\begin{array}{c}\text { Concentration } \\
(\mu \mathrm{g} / \mathrm{mL})\end{array}$ & Solven \\
\hline AM2201 2'-naphthyl isomer & $\mathrm{C}_{24} \mathrm{H}_{22} \mathrm{FNO}$ & 1000 & DMSO \\
\hline JWH 018 2'-naphthyl isomer & $\mathrm{C}_{24} \mathrm{H}_{23} \mathrm{NO}$ & 1000 & DMSO \\
\hline JWH 073 2'-naphthyl-N-(1,1-dimethylethyl) isomer & $\mathrm{C}_{23} \mathrm{H}_{21} \mathrm{NO}$ & 1000 & DMSO \\
\hline JWH 200 2'-naphthyl isomer & $\mathrm{C}_{25} \mathrm{H}_{24} \mathrm{~N}_{2} \mathrm{O}_{2}$ & 1000 & DMSO \\
\hline$( \pm)$ WIN 55212 (mesylate) & $\mathrm{C}_{27} \mathrm{H}_{26} \mathrm{~N}_{2} \mathrm{O}_{3}$ & 1000 & DMSO \\
\hline AM1235 & $\mathrm{C}_{24} \mathrm{H}_{21} \mathrm{FN}_{2} \mathrm{O}_{3}$ & 1000 & DMSO \\
\hline JWH 018 N-(5-bromopentyl) analog & $\mathrm{C}_{24} \mathrm{H}_{22} \mathrm{BrNO}$ & 1000 & DMSO \\
\hline JWH 072 & $\mathrm{C}_{22} \mathrm{H}_{19} \mathrm{NO}$ & 1000 & DMSO \\
\hline JWH 073 4-methylnaphthyl analog & $\mathrm{C}_{24} \mathrm{H}_{23} \mathrm{NO}$ & 1000 & DMSO \\
\hline URB754 & $\mathrm{C}_{16} \mathrm{H}_{14} \mathrm{~N}_{2} \mathrm{O}_{2}$ & 1000 & DMSO \\
\hline WIN 54461 & $\mathrm{C}_{23} \mathrm{H}_{25} \mathrm{BrN}_{2} \mathrm{O}_{3}$ & 1000 & DMSO \\
\hline Cannabipiperidiethanone & $\mathrm{C}_{24} \mathrm{H}_{28} \mathrm{~N}_{2} \mathrm{O}_{2}$ & 2500 & $\mathrm{EtOH}$ \\
\hline
\end{tabular}

Solution based standards were made using different organic solvents, including acetonitrile $(\mathrm{ACN})$, ethanol $(\mathrm{EtOH})$, methanol $(\mathrm{MeOH})$, and methyl acetate $(\mathrm{MeOAc})$, at different concentrations as shown in Table 3. Each stock solution was further diluted to $10 \mu \mathrm{g} / \mathrm{mL}$ in methanol to be used as a working standard solution. Methanol and formic 
acid were both Optima ${ }^{\circledR}$ LC/MS grade and purchased from Fisher Scientific (Fair Lawn, NJ, USA). Proadifen was purchased from Sigma-Aldrich (St Louis, MO, USA) and prepared in methanol to a concentration of $10 \mu \mathrm{g} / \mathrm{mL}$. Proadifen was used in order to lock the retention time in the GC system.

Table 3. List of solution based NPS standards with different solvents.

\begin{tabular}{|c|c|c|c|}
\hline NPS & $\begin{array}{c}\text { Molecular } \\
\text { Formula }\end{array}$ & $\begin{array}{c}\text { Concentration } \\
(\mu \mathrm{g} / \mathrm{mL})\end{array}$ & Solvent \\
\hline ( \pm )5-ері CP 55,940 & $\mathrm{C}_{24} \mathrm{H}_{40} \mathrm{O}_{3}$ & 10000 & $\mathrm{MeOAc}$ \\
\hline ( \pm )-epi CP 47, 497 & $\mathrm{C}_{21} \mathrm{H}_{34} \mathrm{O}_{2}$ & 1000 & $\mathrm{MeOH}$ \\
\hline AM2201 N-(2-fluoropentyl) isomer & $\mathrm{C}_{24} \mathrm{H}_{22} \mathrm{FNO}$ & 1000 & $\mathrm{ACN}$ \\
\hline AM2201 N-(3-fluoropentyl) isomer & $\mathrm{C}_{24} \mathrm{H}_{22} \mathrm{FNO}$ & 1000 & $\mathrm{MeOH}$ \\
\hline AM2201 N-(4-fluoropentyl) isomer & $\mathrm{C}_{24} \mathrm{H}_{22} \mathrm{FNO}$ & 1000 & $\mathrm{MeOH}$ \\
\hline AM2232 & $\mathrm{C}_{24} \mathrm{H}_{20} \mathrm{~N}_{2} \mathrm{O}$ & 10000 & $\mathrm{ACN}$ \\
\hline AM694 4-iodo isomer & $\mathrm{C}_{20} \mathrm{H}_{19} \mathrm{FINO}$ & 10000 & $\mathrm{MeOH}$ \\
\hline HU-308 & $\mathrm{C}_{27} \mathrm{H}_{42} \mathrm{O}_{3}$ & 10000 & $\mathrm{MeOAc}$ \\
\hline JWH 007 & $\mathrm{C}_{25} \mathrm{H}_{25} \mathrm{NO}$ & 10000 & $\mathrm{MeOH}$ \\
\hline JWH 011 & $\mathrm{C}_{27} \mathrm{H}_{29} \mathrm{NO}$ & 10000 & $\mathrm{MeOH}$ \\
\hline JWH 016 & $\mathrm{C}_{24} \mathrm{H}_{23} \mathrm{NO}$ & 10000 & $\mathrm{MeOH}$ \\
\hline JWH 018 & $\mathrm{C}_{24} \mathrm{H}_{23} \mathrm{NO}$ & 10000 & $\mathrm{MeOH}$ \\
\hline JWH 018 2'-naphthyl-N-(1-methylbutyl) isomer & $\mathrm{C}_{24} \mathrm{H}_{23} \mathrm{NO}$ & 10000 & $\mathrm{MeOH}$ \\
\hline JWH 018 2'-naphthyl-N-(2-methylbutyl) isomer & $\mathrm{C}_{24} \mathrm{H}_{23} \mathrm{NO}$ & 10000 & $\mathrm{MeOH}$ \\
\hline JWH 018 2'-naphthyl-N-(3-methylbutyl) isomer & $\mathrm{C}_{24} \mathrm{H}_{23} \mathrm{NO}$ & 10000 & $\mathrm{MeOH}$ \\
\hline JWH 018 6-methoxyindole analog & $\mathrm{C}_{25} \mathrm{H}_{25} \mathrm{NO}_{2}$ & 10000 & $\mathrm{MeOH}$ \\
\hline JWH 018 N-(1-methylbutyl) isomer & $\mathrm{C}_{24} \mathrm{H}_{23} \mathrm{NO}$ & 10000 & $\mathrm{MeOH}$ \\
\hline JWH 018 N-(2-methylbutyl) isomer & $\mathrm{C}_{24} \mathrm{H}_{23} \mathrm{NO}$ & 10000 & $\mathrm{MeOH}$ \\
\hline JWH $018 \mathrm{~N}$-(3-methylbutyl) isomer & $\mathrm{C}_{24} \mathrm{H}_{23} \mathrm{NO}$ & 5000 & $\mathrm{MeOH}$ \\
\hline JWH 018 N-(4,5-epoxypentyl) analog & $\mathrm{C}_{24} \mathrm{H}_{21} \mathrm{NO}_{2}$ & 10000 & $\mathrm{MeOH}$ \\
\hline JWH 022 & $\mathrm{C}_{24} \mathrm{H}_{21} \mathrm{NO}_{2}$ & 10000 & $\mathrm{MeOH}$ \\
\hline JWH 030 & $\mathrm{C}_{20} \mathrm{H}_{21} \mathrm{NO}$ & 10000 & $\mathrm{MeOH}$ \\
\hline JWH 031 & $\mathrm{C}_{21} \mathrm{H}_{23} \mathrm{NO}$ & 10000 & $\mathrm{MeOH}$ \\
\hline JWH 073 2-methylnaphthyl analog & $\mathrm{C}_{24} \mathrm{H}_{23} \mathrm{NO}$ & 10000 & $\mathrm{MeOH}$ \\
\hline JWH 073 2'-naphthyl-N-(1-methylpropyl) isomer & $\mathrm{C}_{23} \mathrm{H}_{21} \mathrm{NO}$ & 10000 & $\mathrm{MeOH}$ \\
\hline
\end{tabular}




\begin{tabular}{|c|c|c|c|}
\hline NPS & $\begin{array}{c}\text { Molecular } \\
\text { Formula }\end{array}$ & $\begin{array}{c}\text { Concentration } \\
(\mu \mathrm{g} / \mathrm{mL})\end{array}$ & Solvent \\
\hline JWH 081 3-methoxynaphthyl isomer & $\mathrm{C}_{25} \mathrm{H}_{25} \mathrm{NO}_{2}$ & 10000 & $\mathrm{MeOH}$ \\
\hline JWH 081 6-methoxynaphthyl isomer & $\mathrm{C}_{25} \mathrm{H}_{25} \mathrm{NO}_{2}$ & 10000 & $\mathrm{MeOH}$ \\
\hline JWH 081 7-methoxynaphthyl isomer & $\mathrm{C}_{25} \mathrm{H}_{25} \mathrm{NO}_{2}$ & 10000 & $\mathrm{MeOH}$ \\
\hline JWH 081 8-methoxynaphthyl isomer & $\mathrm{C}_{25} \mathrm{H}_{25} \mathrm{NO}_{2}$ & 10000 & $\mathrm{MeOH}$ \\
\hline JWH 122 2-methylnaphthyl isomer & $\mathrm{C}_{25} \mathrm{H}_{25} \mathrm{NO}$ & 10000 & $\mathrm{MeOH}$ \\
\hline JWH 122 3-methylnaphthyl isomer & $\mathrm{C}_{25} \mathrm{H}_{25} \mathrm{NO}$ & 10000 & $\mathrm{MeOH}$ \\
\hline JWH 122 6-methylnaphthyl isomer & $\mathrm{C}_{25} \mathrm{H}_{25} \mathrm{NO}$ & 10000 & $\mathrm{MeOH}$ \\
\hline JWH 122 8-methylnaphthyl isomer & $\mathrm{C}_{25} \mathrm{H}_{25} \mathrm{NO}$ & 10000 & $\mathrm{MeOH}$ \\
\hline JWH 145 & $\mathrm{C}_{26} \mathrm{H}_{25} \mathrm{NO}$ & 10000 & $\mathrm{MeOH}$ \\
\hline JWH 175 & $\mathrm{C}_{24} \mathrm{H}_{25} \mathrm{~N}$ & 10000 & $\mathrm{ACN}$ \\
\hline JWH 201 & $\mathrm{C}_{22} \mathrm{H}_{25} \mathrm{NO}_{2}$ & 10000 & $\mathrm{MeOAc}$ \\
\hline JWH 203 3-chlorophenyl isomer & $\mathrm{C}_{21} \mathrm{H}_{22} \mathrm{ClNO}$ & 10000 & $\mathrm{MeOH}$ \\
\hline JWH 210 2-ethylnaphthyl isomer & $\mathrm{C}_{26} \mathrm{H}_{27} \mathrm{NO}$ & 1000 & $\mathrm{MeOH}$ \\
\hline JWH 210 3-ethylnaphthyl isomer & $\mathrm{C}_{26} \mathrm{H}_{27} \mathrm{NO}$ & 10000 & $\mathrm{MeOH}$ \\
\hline JWH 210 5-ethylnaphthyl isomer & $\mathrm{C}_{26} \mathrm{H}_{27} \mathrm{NO}$ & 1000 & $\mathrm{MeOH}$ \\
\hline JWH 210 6-ethylnaphthyl isomer & $\mathrm{C}_{26} \mathrm{H}_{27} \mathrm{NO}$ & 10000 & $\mathrm{MeOH}$ \\
\hline JWH 210 8-ethylnaphthyl isomer & $\mathrm{C}_{26} \mathrm{H}_{27} \mathrm{NO}$ & 10000 & $\mathrm{MeOH}$ \\
\hline JWH 251 & $\mathrm{C}_{22} \mathrm{H}_{25} \mathrm{NO}$ & 10000 & $\mathrm{MeOAc}$ \\
\hline JWH 251 3-methylphenyl isomer & $\mathrm{C}_{22} \mathrm{H}_{25} \mathrm{NO}$ & 10000 & $\mathrm{MeOH}$ \\
\hline JWH 251 4-methylphenyl isomer & $\mathrm{C}_{22} \mathrm{H}_{25} \mathrm{NO}$ & 10000 & $\mathrm{MeOH}$ \\
\hline JWH 302 & $\mathrm{C}_{22} \mathrm{H}_{25} \mathrm{NO}_{2}$ & 10000 & $\mathrm{MeOAc}$ \\
\hline JWH 309 & $\mathrm{C}_{30} \mathrm{H}_{27} \mathrm{NO}$ & 10000 & $\mathrm{MeOAc}$ \\
\hline JWH 369 & $\mathrm{C}_{26} \mathrm{H}_{24} \mathrm{ClNO}$ & 10000 & $\mathrm{MeOH}$ \\
\hline JWH 370 & $\mathrm{C}_{27} \mathrm{H}_{27} \mathrm{NO}$ & 10000 & $\mathrm{MeOH}$ \\
\hline JWH 398 & $\mathrm{C}_{24} \mathrm{H}_{22} \mathrm{ClNO}$ & 10000 & $\mathrm{MeOH}$ \\
\hline JWH 398 2-chloronaphthyl isomer & $\mathrm{C}_{24} \mathrm{H}_{22} \mathrm{ClNO}$ & 1000 & $\mathrm{MeOH}$ \\
\hline JWH 398 8-chloronaphthyl isomer & $\mathrm{C}_{24} \mathrm{H}_{22} \mathrm{ClNO}$ & 10000 & $\mathrm{MeOH}$ \\
\hline JWH 424 & $\mathrm{C}_{24} \mathrm{H}_{22} \mathrm{BrNO}$ & 10000 & $\mathrm{MeOH}$ \\
\hline Norsufentanil & $\mathrm{C}_{16} \mathrm{H}_{24} \mathrm{~N}_{2} \mathrm{O}_{2}$ & 10000 & $\mathrm{MeOH}$ \\
\hline Pyrovalerone & $\mathrm{C}_{16} \mathrm{H}_{23} \mathrm{NO}$ & 10000 & $\mathrm{MeOH}$ \\
\hline RCS-4 2-methoxy isomer & $\mathrm{C}_{21} \mathrm{H}_{23} \mathrm{NO}_{2}$ & 10000 & $\mathrm{MeOH}$ \\
\hline RCS-4 3-methoxy isomer & $\mathrm{C}_{21} \mathrm{H}_{23} \mathrm{NO}_{2}$ & 10000 & $\mathrm{MeOH}$ \\
\hline TMA-2 & $\mathrm{C}_{12} \mathrm{H}_{19} \mathrm{NO}_{3}$ & 10000 & $\mathrm{EtOH}$ \\
\hline
\end{tabular}




\subsection{Ion Mobility Spectrometry}

A commercial IMS instrument, Barringer IONSCAN 400B (currently, Smiths Detection IONSCAN 400B, Mississauga, ON, Cananda), was used under factory default operating conditions as shown in Table 4. A pre-conditioned Teflon membrane substrate (Filter stock No. 11510, Barringer) was used to introduce each analyte into the thermal desorption chamber. Sample delivery was achieved by spiking $10 \mathrm{ng}(1 \mu \mathrm{L}$ of $10 \mu \mathrm{g} / \mathrm{mL}$ standard solution) of a standard onto the membrane and drying it for $30 \mathrm{sec}$. The experimental drift time and the calculated reduced mobility $\left(K_{0}\right)$ values from each standard were determined and programed into a detection menu for the substances of interest using the software Instrument Manager 5.052 (Smiths Detection). The detailed detection alarm parameters are as follows: full-width at the half maximum height (FWHM) of 380, peak amplitude of 50, and threshold of 2.5. With the exception of 8 NPS (30 for MDPV, pentylone, bk-EBDB, 4'-MePHP, XLR-11, and 25I-NBOMe; 25 for 2,3-pentylone, bk-DMBDB), the variability for most of the NPS investigated was 50 .

Table 4. Operating condition for the Barringer IONSCAN 400B.

\begin{tabular}{cc}
\hline Operating mode & Narcotic mode (positive) \\
Desorber temperature & $300{ }^{\circ} \mathrm{C}$ \\
Inlet temperature & $250^{\circ} \mathrm{C}$ \\
Drift tube temperature & $235^{\circ} \mathrm{C}$ \\
Drift flow & $300 \mathrm{~mL} / \mathrm{min}$ \\
Shutter grid width & $0.200 \mathrm{~ms}$ \\
Scan period & $20 \mathrm{~ms}$ \\
Dopant & Nicotinamide \\
\hline
\end{tabular}




\subsection{Direct Analysis in Real Time Quadrupole Time-of-Flight}

An IonSense DART®-SVP (Saugus, MA, USA) ionization source was coupled to a high resolution Agilent 6530 Accurate-Mass QTOF mass analyzer (Santa Clara, CA, USA). The DART source was operated under the following conditions: positive mode, helium as a reagent gas at $350{ }^{\circ} \mathrm{C}$ with a flow rate of $3 \mathrm{~L} / \mathrm{min}$ and a sample speed of $1.5 \mathrm{~mm} / \mathrm{sec}$. Accurate mass spectral data was collected in positive mode with the mass range of $\mathrm{m} / \mathrm{z} 50$ to 600 . The fragmentor voltage was $250 \mathrm{~V}$ and different collision energies $(10,20$, and $40 \mathrm{eV})$ were applied in the auto MS/MS mode. A linear rail system with the 1D transmission mode was utilized to ensure the consistent delivery of samples. The distance between the DART source and the inlet of mass analyzer was set at $3 \mathrm{~cm}$.

\subsection{Gas Chromatography Tandem Mass Spectrometry}

An Agilent 7890A GC system (Santa Clara, CA, USA) with a DB-5MS column $(30 \mathrm{~m} \times 250 \mu \mathrm{m} \times 0.25 \mu \mathrm{m}, \mathrm{J} \& \mathrm{~W}$, Agilent) was coupled to an Agilent 7000 GC-MS Triple Quad mass spectrometer. In the gas chromatography tandem mass spectrometry (GCMS/MS) system, the retention-time locking (RTL) with proadifen (20.765 min) was used to provide a consistent retention time for the analytes over time. The GC parameters for full scan mode were as follows: injection volume of $5 \mu \mathrm{L}$, split ratio of 10:1, injection temperature of $280{ }^{\circ} \mathrm{C}$, transfer line temperature of $300{ }^{\circ} \mathrm{C}$, and helium as a carrier gas at different flow rates depending on the pressure required for the RTL. The oven temperature program was initiated at $60{ }^{\circ} \mathrm{C}$, held for 1 minute and then ramped at the rate of $10{ }^{\circ} \mathrm{C} / \mathrm{min}$ to a maximum of $325{ }^{\circ} \mathrm{C}$, where the temperature was held for 8 minutes. The operating parameters for MS in full scan mode were as follows: gain factor of 1 , 
source temperature at $300{ }^{\circ} \mathrm{C}$, quadrupole temperature at $150{ }^{\circ} \mathrm{C}$, solvent delay of 3.75 min, ionization energy of $70 \mathrm{eV}$, and acquisition mass range of $\mathrm{m} / \mathrm{z} 45-570$. In CI mode, methane was used as a reagent gas with a flow of $20 \%$. In the product ion scan and multiple reaction monitoring $(\mathrm{MRM})$ mode, the flow rate of nitrogen as the collision gas and helium as the quench gas was $1.0 \mathrm{~mL} / \mathrm{min}$. The acquisition mass range was varied in product ion scan mode during MRM optimization starting from $\mathrm{m} / \mathrm{z} 35$ up to the mass of the selected precursor ion. Other MS operating conditions remained the same.

\subsection{Gas Chromatography Quadrupole Time-of-Flight}

A high-resolution 7200 Accurate-Mass Q-TOF mass spectrometer (Santa Clara, CA, USA) was coupled to an Agilent 7890B GC system with a DB-5MS column (30m× $250 \mu \mathrm{m} \times 0.25 \mu \mathrm{m}, \mathrm{J} \& \mathrm{~W}$, Agilent). With the exception of an injection volume of $1 \mu \mathrm{L}$ with splitless mode in CI source and transfer line temperature of $325{ }^{\circ} \mathrm{C}$, the operating parameters for the GC system remained consistent with those used for GC-MS/MS. The operating parameters for MS in the full scan mode were as follows: source temperature at $300{ }^{\circ} \mathrm{C}$, quadrupole temperature at $150{ }^{\circ} \mathrm{C}$, solvent delay of 4 min, acquisition mass range of $\mathrm{m} / \mathrm{z} 50$ to 500, and acquisition time of $200 \mathrm{~ms} / \mathrm{spectrum}$. Methane was used as a reagent gas for $\mathrm{CI}$ mode $(90 \mathrm{eV})$. In targeted MS/MS mode, different collision energies $(10,20$, and $40 \mathrm{eV})$ were applied for pre-programed target masses of analytes using an acquisition time of $500 \mathrm{~ms} / \mathrm{spectrum}$. Other MS operating conditions remained the same as the full scan mode. The daily mass calibration was performed using mass spectrometric grade perfluorotributylamine (PFTBA) in order to achieve high mass accuracy. 


\subsection{Electrospray Ionization Ion Mobility Spectrometry Mass Spectrometry}

A commercial Excellims RA4100 ESI-IMS-MS (Acton, MA, USA) was equipped with an Extrel QC-150 quadrupole mass spectrometer (Pittsburgh, PA, USA). The instrument was operated in positive ion mode under the following conditions: $3.2 \mathrm{kV}$ above the drift tube potential for an ESI source voltage, drift tube voltage of $8 \mathrm{kV}$ at a temperature of $150{ }^{\circ} \mathrm{C}$, ultra high purity air as a drift gas at a flow rate of $2 \mathrm{~L} / \mathrm{min}$, and a mass range of $m / z 50$ to 500. The sample was infused using a Harvard Apparatus Model 22 syringe pump (Holliston, MA, USA) fitted with a $250 \mu \mathrm{L}$ Hamilton Gas tight syringe (Reno, NV, USA) at a flow rate of $3 \mu \mathrm{L} / \mathrm{min}$. 


\section{CHAPTER 4. DEVELOPMENT OF RAPID SCREENING METHODS FOR NPS}

\subsection{Qualitative Analysis of NPS by Ion Mobility Spectrometry}

A commercial Barringer IMS equipped with a ${ }^{63} \mathrm{Ni}$ radioactive ion source (Figure 19) was utilized for the qualitative analysis of NPS. As previously mentioned, this instrument provides a number of advantages including ease of operation and maintenance, high sensitivity, fast response, and high throughput analysis. With these benefits, the instrument was evaluated for the rapid detection and characterization of emerging NPS. Since this IMS analyzes compounds in gas phase, samples are heated first to increase the volume of vapors during the sample introduction at the desorber [114]. The inlet and drift tube are also kept in the high temperature to prevent the condensation of vaporized analytes from the desorber [114]. In the current investigation, the factory setting for the narcotic mode (positive) was kept because previous studies showed that some of these emerging NPS were not temperature dependent in the range from 190 to $280{ }^{\circ} \mathrm{C}$ for the desorber and from 200 to $290{ }^{\circ} \mathrm{C}$ for the inlet [115].

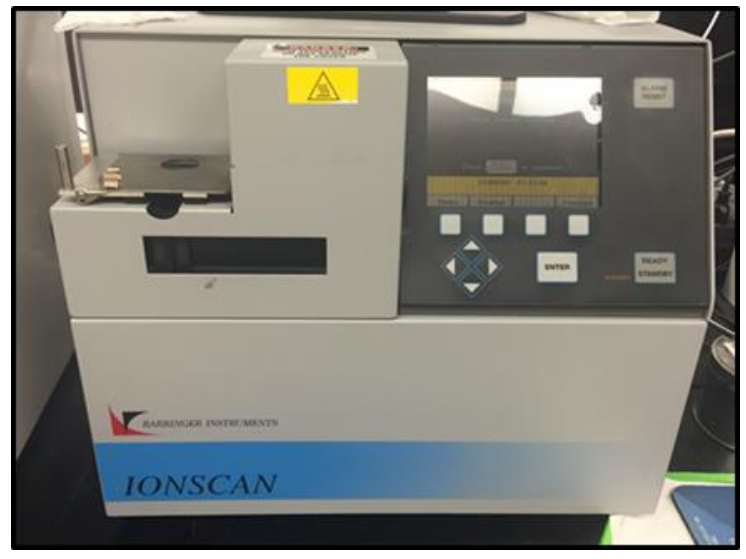

Figure 19. Picture of Barringer IONSCAN 400B. 


\subsubsection{Programing of Parameters and Detection of NPS}

Table 5 shows the list of 35 NPS that were successfully detected and characterized in our laboratory using the Barringer IMS. The experimental drift time for each substance were determined ranging from 11.741 to $19.092 \mathrm{~ms}$, which correspond to the $K_{0}$ values ranging from 1.5373 to $0.9466 \mathrm{~cm}^{2} / \mathrm{Vs}$. The detection menu for each compound was then programmed into the acquisition method following the results obtained in Table 5. The comparison between our results and previously reported $K o$ values from other research groups (Armenta et al. and Joshi et al.) showed good agreement for MDPV, methylone, $\alpha-\mathrm{PVP}$, 3-FMC, 4-FMC, 4-MEC, 4-MMC, buphedrone, 4'-MePPP, butylone, naphyrone, and UR-144 with differences less than \pm $0.006 \mathrm{~cm}^{2} / \mathrm{Vs}$ [115-116]. The $K_{0}$ value for methylone were identical, while 4-MMC and UR-144 showed the negligible difference (0.006) between research groups. The good consistency is mainly because the same type of instrument was used in the detection and characterization of these NPS although the instrument settings were slightly different.

That being said, there were some discrepancies of the $K_{0}$ value for methylone, 4MEC, 4-MMC, buphedrone, 4'-MePPP, and butylone between our results from the ${ }^{63} \mathrm{Ni}$ IMS and the previously reported $K o$ values from the different ionization sources, ESI and APCI IMS-MS as shown in Table 5 [117-118]. The discrepancies might be because the reference standard was not considered to determine the $K_{0}$ values when they reported these values from other ionization sources as described in Equation 4. Different ionized species may have been produced during the ionization process by the ESI and APCI source. It is understood that protonated molecular ions are produced in the Barringer 
IMS as a result of the ionization process governed by the reactions between reactant ions, a reagent gas, and analytes. Unfortunately, the ionized species from the Barringer IMS cannot be identified because of the lack of identification capabilities in this instrument.

Table 5. List of 35 NPS analyzed by the Barringer IMS and the results from the study [119].

\begin{tabular}{|c|c|c|c|c|}
\hline NPS & $\begin{array}{l}\text { Molecular } \\
\text { Formula }\end{array}$ & $\begin{array}{c}\text { Drift } \\
\text { time }(\mathrm{ms})\end{array}$ & $\begin{array}{c}K_{0} \\
\left(\mathrm{~cm}^{2} / \mathrm{Vsec}\right)\end{array}$ & Reference $K_{0}\left(\mathrm{~cm}^{2} / \mathrm{Vsec}\right)$ \\
\hline \multicolumn{5}{|c|}{ Synthetic cathinones } \\
\hline 2,3-MDPV & $\mathrm{C}_{16} \mathrm{H}_{21} \mathrm{NO}_{3}$ & 14.897 & 1.2150 & \\
\hline MDPV & $\mathrm{C}_{16} \mathrm{H}_{21} \mathrm{NO}_{3}$ & 15.005 & 1.2026 & $1.199[115], 1.201[116], 1.18[117]$ \\
\hline 2,3-MDMC & $\mathrm{C}_{11} \mathrm{H}_{13} \mathrm{NO}_{3}$ & 12.554 & 1.4411 & \\
\hline Methylone & $\mathrm{C}_{11} \mathrm{H}_{13} \mathrm{NO}_{3}$ & 12.570 & 1.4354 & $1.435[115], 1.435[116], 1.28^{*}[117]$ \\
\hline 2,3-Pentylone & $\mathrm{C}_{13} \mathrm{H}_{17} \mathrm{NO}_{3}$ & 13.511 & 1.3379 & \\
\hline Pentylone & $\mathrm{C}_{13} \mathrm{H}_{17} \mathrm{NO}_{3}$ & 13.677 & 1.3217 & \\
\hline bk-DMBDB & $\mathrm{C}_{13} \mathrm{H}_{17} \mathrm{NO}_{3}$ & 13.553 & 1.3345 & \\
\hline bk-EBDB & $\mathrm{C}_{13} \mathrm{H}_{17} \mathrm{NO}_{3}$ & 13.627 & 1.3274 & \\
\hline 2-MePBP & $\mathrm{C}_{15} \mathrm{H}_{21} \mathrm{NO}$ & 13.726 & 1.3170 & \\
\hline 3-MePBP & $\mathrm{C}_{15} \mathrm{H}_{21} \mathrm{NO}$ & 13.979 & 1.2928 & \\
\hline 4-MePBP & $\mathrm{C}_{15} \mathrm{H}_{21} \mathrm{NO}$ & 13.879 & 1.3024 & \\
\hline$\alpha-P V P$ & $\mathrm{C}_{15} \mathrm{H}_{21} \mathrm{NO}$ & 13.802 & 1.3094 & $1.306[115]$ \\
\hline 3-FMC & $\mathrm{C}_{10} \mathrm{H}_{12} \mathrm{FNO}$ & 11.742 & 1.5271 & $1.530[115]$ \\
\hline 4-FMC & $\mathrm{C}_{10} \mathrm{H}_{12} \mathrm{FNO}$ & 11.741 & 1.5373 & $1.539[116]$ \\
\hline 4-MEC & $\mathrm{C}_{12} \mathrm{H}_{17} \mathrm{NO}$ & 12.520 & 1.4418 & $1.447[115], 1.30^{\dagger}[117], 1.41[118]$ \\
\hline Pentedrone & $\mathrm{C}_{12} \mathrm{H}_{17} \mathrm{NO}$ & 12.463 & 1.4504 & \\
\hline 4-MMC & $\mathrm{C}_{11} \mathrm{H}_{15} \mathrm{NO}$ & 12.008 & 1.5050 & $1.499[116]$ \\
\hline Buphedrone & $\mathrm{C}_{11} \mathrm{H}_{15} \mathrm{NO}$ & 11.893 & 1.5199 & $1.520[116]$ \\
\hline 4'-MeOPPP & $\mathrm{C}_{14} \mathrm{H}_{19} \mathrm{NO}_{2}$ & 13.677 & 1.3194 & \\
\hline 4'-MePHP & $\mathrm{C}_{17} \mathrm{H}_{25} \mathrm{NO}$ & 15.068 & 1.1977 & \\
\hline 4'-MePPP & $\mathrm{C}_{14} \mathrm{H}_{19} \mathrm{NO}$ & 13.342 & 1.3547 & $1.350[115], 1.33[118]$ \\
\hline bk-MDDMA & $\mathrm{C}_{12} \mathrm{H}_{15} \mathrm{NO}_{3}$ & 13.010 & 1.3903 & \\
\hline bk-MDEA & $\mathrm{C}_{12} \mathrm{H}_{15} \mathrm{NO}_{3}$ & 13.116 & 1.3790 & \\
\hline
\end{tabular}




\begin{tabular}{ccccc}
\hline NPS & $\begin{array}{c}\text { Molecular } \\
\text { Formula }\end{array}$ & $\begin{array}{c}\text { Drift } \\
\text { time }(\mathrm{ms})\end{array}$ & $\begin{array}{c}K_{0} \\
\left(\mathrm{~cm}^{2} / \mathrm{Vsec}\right)\end{array}$ & Reference $K_{0}\left(\mathrm{~cm}^{2} / \mathrm{Vsec}\right)$ \\
Butylone & $\mathrm{C}_{12} \mathrm{H}_{15} \mathrm{NO}_{3}$ & 13.005 & 1.3808 & $1.382[115], 1.380[116], 1.34[118]$ \\
Naphyrone & $\mathrm{C}_{19} \mathrm{H}_{23} \mathrm{NO}$ & 15.671 & 1.1532 & $1.152[116]$ \\
$\alpha$-Naphyrone & $\mathrm{C}_{19} \mathrm{H}_{23} \mathrm{NO}$ & 15.433 & 1.1710 & \\
\hline \hline Synthetic cannabinoids & & & \\
AM1220 & $\mathrm{C}_{26} \mathrm{H}_{26} \mathrm{~N}_{2} \mathrm{O}$ & 18.282 & 0.9885 & \\
AM2233 & $\mathrm{C}_{22} \mathrm{H}_{23} \mathrm{IN} \mathrm{N}_{2} \mathrm{O}$ & 17.786 & 1.0161 & \\
CPE & $\mathrm{C}_{24} \mathrm{H}_{28} \mathrm{~N}_{2} \mathrm{O} 2$ & 18.149 & 0.9959 & \\
JWH-018 & $\mathrm{C}_{24} \mathrm{H}_{23} \mathrm{NO}$ & 17.575 & 1.0283 & \\
APICA & $\mathrm{C}_{24} \mathrm{H}_{32} \mathrm{~N}_{2} \mathrm{O}$ & 19.092 & 0.9466 & $1.042[115]$ \\
MAM2201 & $\mathrm{C}_{25} \mathrm{H}_{24} \mathrm{FNO}$ & 18.352 & 0.9847 & \\
UR-144 & $\mathrm{C}_{21} \mathrm{H}_{29} \mathrm{NO}$ & 17.239 & 1.0484 & \\
XLR-11 & $\mathrm{C}_{21} \mathrm{H}_{28} \mathrm{FNO}$ & 17.397 & 1.0388 & \\
\hline \hline
\end{tabular}

Phenethylamines

25I-NBOMe $\quad \mathrm{C}_{18} \mathrm{H}_{22} \mathrm{INO}_{3} \quad 17.372 \quad 1.0404$

*: 1.28 for $m / z 208.10$ in APCI and ESI and 1.35 for $m / z 206.08$ in APCI

†: 1.30 for $m / z 192.14$ in APCI and ESI and 1.41 for $m / z, 190.12$ in APCI

Overlaid ion mobility spectra of representative compounds are present in Figure 20. Each substance was clearly determined with a peak at different drift times along with the presence of consistent reactant ion peak (RIP) at $9.675 \mathrm{~ms}$. This RIP plays an important role in proving the consistent performance of instrument as well as to standardize the $K_{0}$ value from the experiments [75]. In the experiment, nicotinamide (NA) was used as a reagent gas producing a reactant ion and the $K_{0}$ values were automatically calculated in the software. As mentioned previously, the rapid detection of analytes (within $20 \mathrm{~ms}$ ) is the biggest advantage of this commercial IMS and it is clearly shown during this investigation with the total analysis time less than one minute. However, the false positive alarm may sound during the analysis of compounds that possess a similar 
reduced mobility. For example, butylone $\left(K_{0}: 1.3808\right)$ and bk-MDEA $\left(K_{0}: 1.3790\right)$ as well as XLR-11 $(K o: 1.0388)$ and 25I-NBOMe $(K o: 1.0404)$ will most likely result in false positive alarms with each other. It was observed that the false positive alarm occurs when the difference of $K_{0}$ is less than 0.008 .

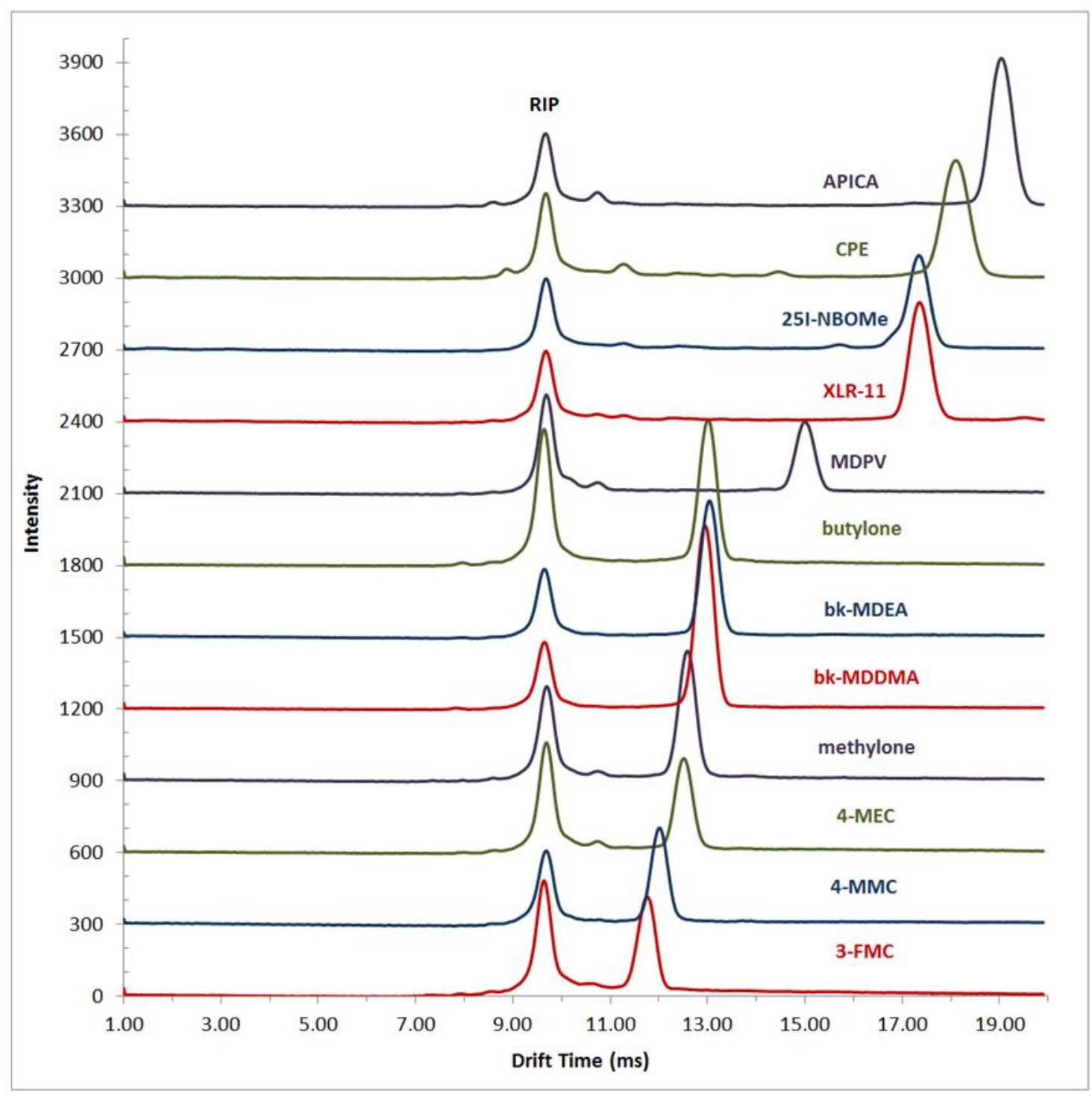

Figure 20. Overlaid ion mobility spectra of the representative NPS by the Barringer IMS [119]. Each substance was distinctly detected with a different drift time and a consistent reactant ion peak (RIP). 
To investigate the separation capability of this Barringer IMS, each mixture was prepared with three different compounds at the concentration of $3.3 \mu \mathrm{g} / \mathrm{mL}$. Two mixtures, which contain 3-FMC, methylone, and MDPV as well as 4-MMC, butylone, and MDPV, were analyzed by the Barringer IMS as shown in Figure 21. Although the baseline separation was only possible when analytes have significantly different $K_{0}$ values, the separation of multiple compounds is still promising. Therefore, this Barringer IMS can be utilized for the rapid screening of mixtures as a presumptive test.
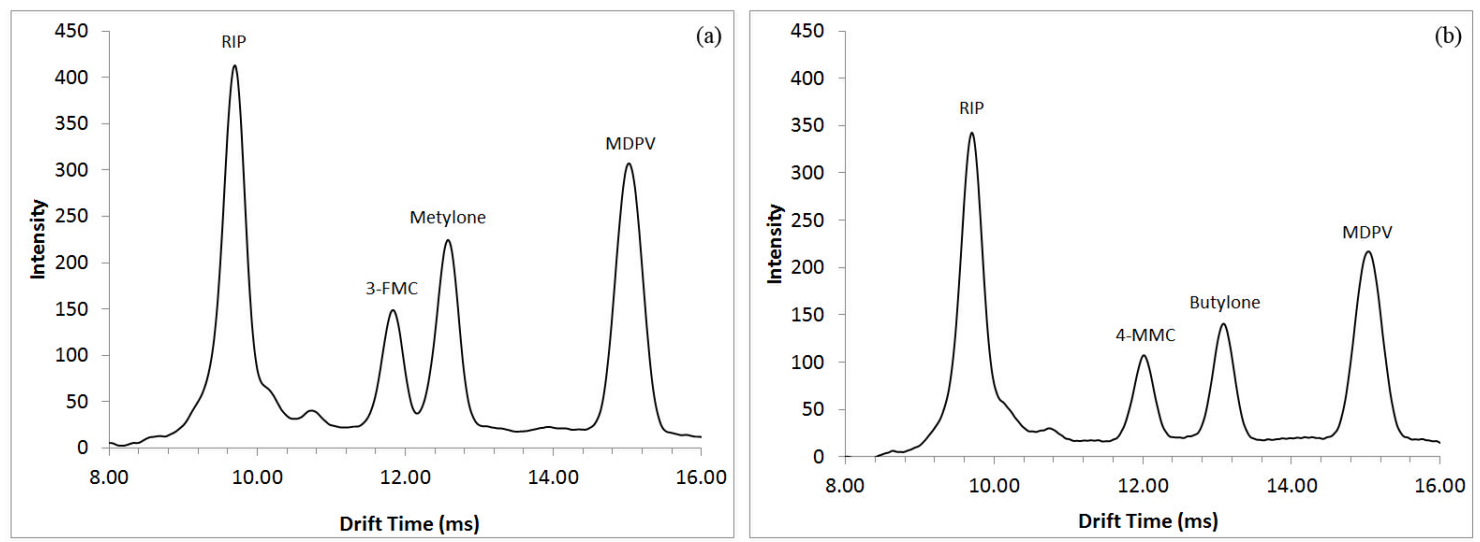

Figure 21. Ion mobility spectra of the two mixtures (a) 3-FMC, methylone, and MDPV, and (b) 4- MMC, butylone, and MDPV at the concentration of $3.3 \mu \mathrm{g} / \mathrm{mL}$ each [119]. The baseline separation was observed when analytes have significantly different $K_{0}$ values only.

\subsubsection{Determination of Detection Limits}

The sensitivity of the instrument is often evaluated by the limits of detection (LODs), which may describe an instrumental signal that is significantly different from the blank or background signal at given concentration [120]. In the investigation of sensitivity, LODs were determined by constructing calibration curves for three NPS 
including bk-EBDB, bk-MDDMA, and APICA as illustrated in Figure 22. Calibration standard solutions were prepared in methanol by serial dilution at the concentration of $0.1,0.25,0.50,1.0,1.5$, and $3.0 \mu \mathrm{g} / \mathrm{mL}$. The measurement for each calibrator was performed 5 times $(\mathrm{n}=5)$ per day after delivering $1 \mu \mathrm{L}$ of each dilution during three consecutive days. The calculation for LODs was determined as three times the standard deviation of the intercept divided by the slope of the linear regression. The detection limits were determined for these substances from 40 to $80 \mathrm{pg}$ in the linear range of 100 to $500 \mathrm{pg}$ with a good linearity, $\mathrm{R}^{2}$ between 0.9831 and 0.9999 .

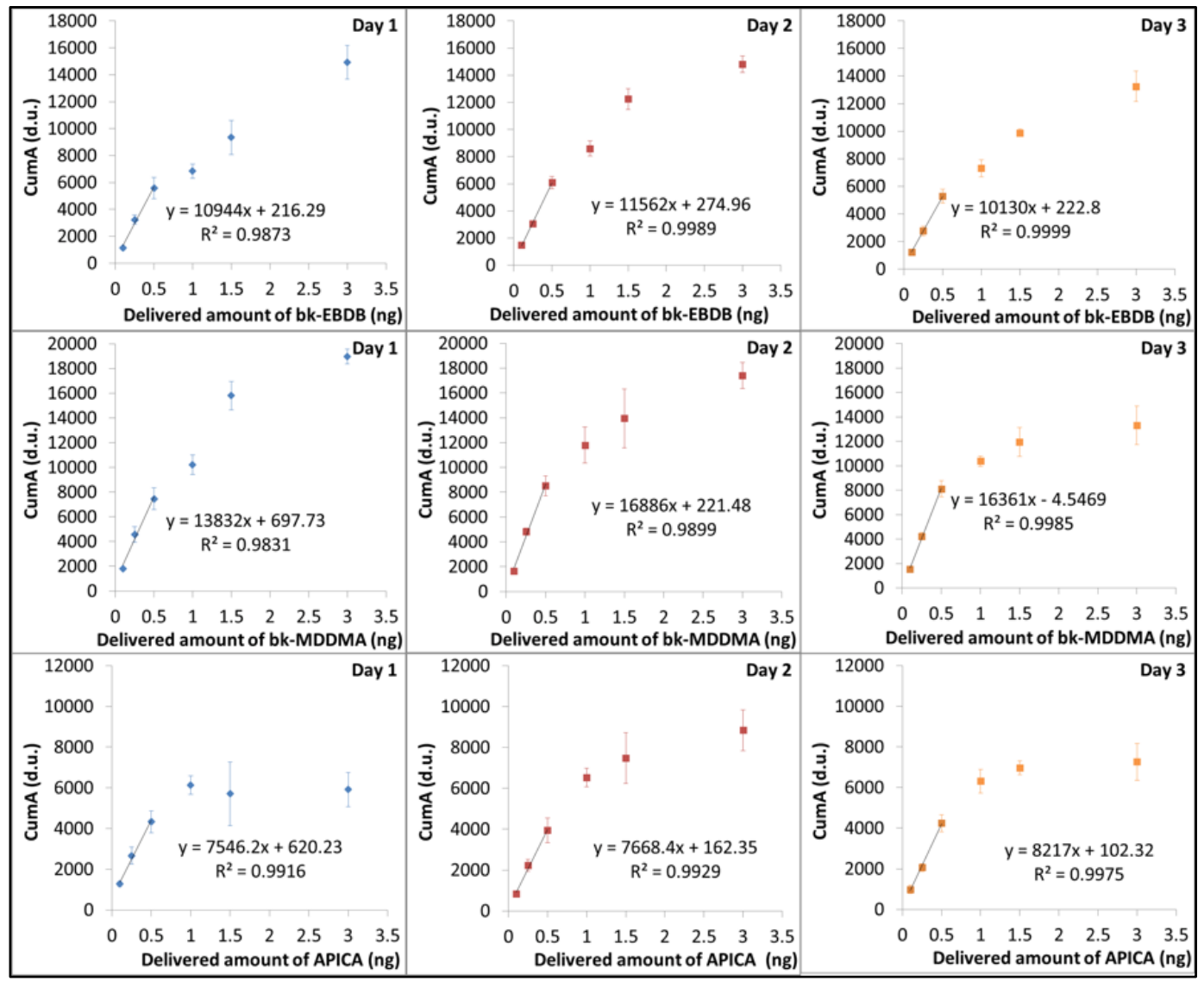

Figure 22. Calibration curves for bk-EBDB (top), bk-MDDMA (middle), and APICA (bottom) in three different days by ${ }^{63} \mathrm{Ni}-\mathrm{IMS}$. Each curve showed a good linearity in the linear range of 100 to $500 \mathrm{pg}$. 
As shown in the calibration curve, the Barringer IMS is often challenged by narrow dynamic linear response because of the depletion of reactant ions along with an increased concentration of analyte molecule [67]. Thus, the application of this Barringer IMS is limited to the qualitative analysis of compounds. In addition, saturation of the drift tube may results in inaccurate measurements and the instrument disability for an extended time because of sample overloading [67]. Moreover, separation capabilities of the Barringer IMS may be limited for some compounds with a resolving power of 36-40 [121]. Nevertheless, IMS is an attractive alternative for the qualitative analysis of these substances of abuse with sub-nanogram detection capability.

\subsubsection{Analysis of Seized Samples}

The applicability of the IMS instrument in actual case scenarios was investigated analyzing seized drug samples provided by a local forensic laboratory. A total number of four seized samples were obtained as solutions after each sample was extracted with methanol. These seized samples were then further diluted with methanol in 1:1000 $(v / v)$ to prevent overloading of analyte in the system. Figure 23 shows overlaid ion mobility spectra of four seized samples analyzed by the Barringer IMS. The corresponding alarms for the identity of samples were obtained as a part of blind test and summarized in Table 6 along with the analysis results from the forensic laboratory. It was informed that the identification of seized samples was confirmed by GC-MS and/or Fourier transform infrared spectroscopy (FTIR). Of the four seized drugs samples, it was found that blind sample 3 contains multiple compounds while the other three samples were discovered to be a single compound. Surprisingly, four positive alarms were obtained for blind sample 
3, which were 4-MePBP, $\alpha$-PVP, bk-MDEA, and butylone. Considering the presence of only two peaks other than the RIP peak in the ion mobility spectrum, it was thought that false positive alarms were attained for the blind sample 3. False positive alarms were mainly because the $K_{0}$ values are similar between $4-\mathrm{MePBP}$ and $\alpha$-PVP as well as bkMDEA and butylone. Likewise, false positive alarms were observed for blind sample 4 , in this case two positive alarms (bk-MDMA and butylone) for only one peak present in the spectrum. Although false positive alarms were obtained for some seized samples, the rapid screening of seized samples was successfully achieved by the Barringer IMS with its simple and ease operation.

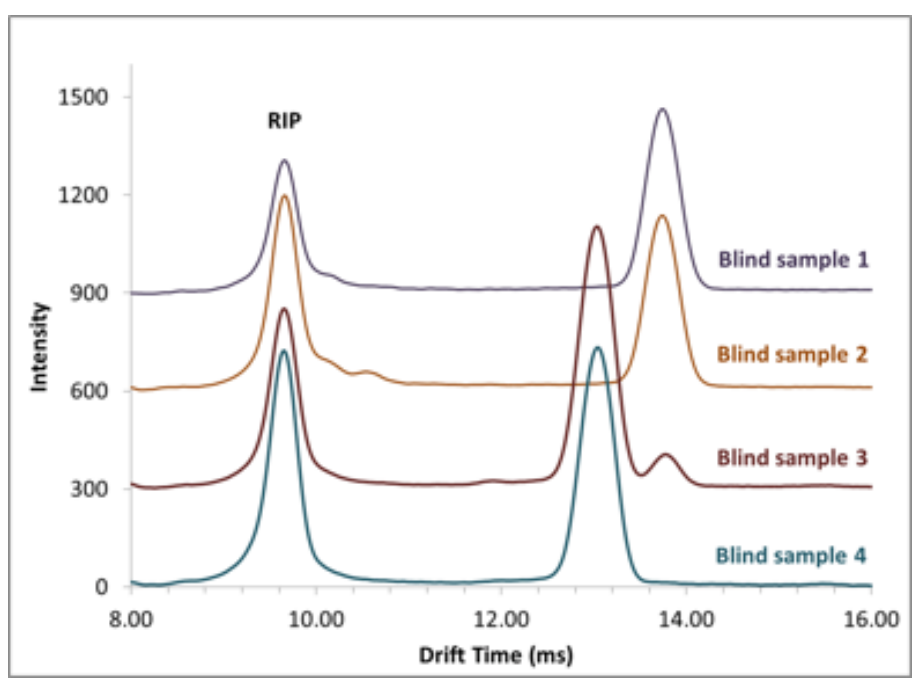

Figure 23. Overlaid ion mobility spectra of four seized drug samples provided by a local forensic laboratory [119]. It was found that blind sample 3 contains multiple compounds. 
Table 6. Results of seized drug sample analysis by the Barringer IMS [119].

\begin{tabular}{|c|c|c|c|c|}
\hline Sample & $\begin{array}{c}\text { Blind } \\
\text { sample } 1\end{array}$ & $\begin{array}{c}\text { Blind } \\
\text { sample } 2\end{array}$ & $\begin{array}{c}\text { Blind } \\
\text { sample } 3\end{array}$ & $\begin{array}{c}\text { Blind } \\
\text { sample } 4\end{array}$ \\
\hline $\begin{array}{c}\text { Results from local } \\
\text { forensic laboratory } \\
\text { (GC-MS and/or FTIR) }\end{array}$ & $\alpha-P V P$ & $\alpha-P V P$ & $\begin{array}{c}\text { bk-MDEA } \\
\alpha \text {-PVP }\end{array}$ & butylone \\
\hline${ }^{63} \mathrm{Ni}-\mathrm{IMS}$ & $\alpha-P V P$ & $\alpha-P V P$ & $\begin{array}{c}\text { 4-MePBP } \\
\alpha-P V P \\
\text { bk-MDEA } \\
\text { butylone }\end{array}$ & $\begin{array}{c}\text { bk-MDEA } \\
\text { butylone }\end{array}$ \\
\hline
\end{tabular}

\subsubsection{Conclusions}

The capability of rapid detection and characterization of emerging NPS were investigated in the section, 4.1. It is observed that emerging NPS were successfully detected with their characteristic reduced mobilities in the default positive mode by the Barringer IMS. The separation of these substances has been also simulated by analyzing mixtures prepared with an equivalent amount of three-analytes. The evaluation of mixture analysis has shown that the baseline separation can be achieved for those compounds that have substantially different drift times. The ability to separate the mixture was also observed for the seized drug sample contained multiple compounds. However, the limitations of the Barringer IMS are the potential false positive results because of the similar reduced mobilities for certain substances, small dynamic range, and lack of identification capability. Nevertheless, successful detection of seized drug samples proved that IMS can be readily used as an alternative rapid screening method for these emerging NPS. As previously demonstrated, the Barringer IMS instrument is 
beneficial in the analysis of these substances with ease of operation and maintenance, relatively inexpensive analysis, and high sensitivity with sub-nanogram detection capabilities. These findings using the Barringer IMS instrument have been reported and published in peer-reviewed literature [119].

\subsection{Direct Analysis in Real Time Quadrupole Time-of-Flight}

This section evaluates the potential for rapid identification of emerging NPS using a direct analysis in real time (DART) ion source coupled to a high-resolution quadrupole time-of-flight mass spectrometer. Although the DART was originally designed for the JEOL AccuTOF time-of-flight mass spectrometer, it is readily coupled to any other mass spectrometers using an atmospheric pressure flange, called Vapur $^{\circledR}$ atmospheric pressure interface by IonSense [85]. As described in Figure 24, the $\operatorname{Vapur}^{\circledR}$ interface consists of a gas-transfer tube and vacuum pump, which provides flexibility to fit the DART source onto different mass spectrometers as well as reproducibility and peak shape for extracted ion profiles.

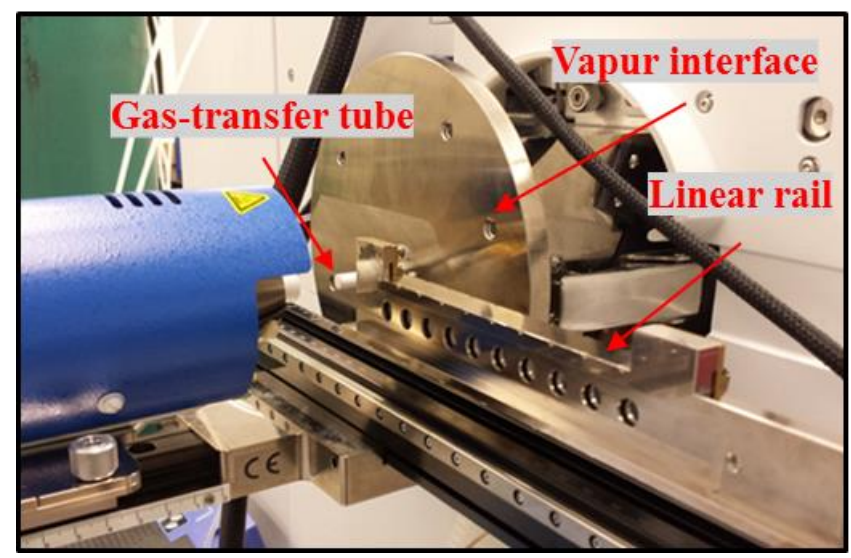

Figure 24. The DART source coupled to a QTOF mass spectrometer with a linear rail system for the sample introduction. 
In DART analysis, samples are introduced by thermal desorption with heated gas in open air [99]. Therefore, it is important to control atmospheric conditions and optimize the instrumental parameters properly. As a relatively new addition to mass spectrometry, DART provides a number of advantages including minimum sample preparation, rapid analysis capability, versatile applications employing different configurations, and the ionization capability for analytes of low to medium molecular mass [100]. However, the acquisition of good spectra is highly dependent on the DART source temperature because analytes are desorbed via a heated gas from the source. In addition, loss of analytes is possible before analysis because of the evaporation of samples at high temperature [100]. Nonetheless, DART-MS has shown great potential for application in the analysis of drugs in the forensic laboratory setting as mentioned previously.

\subsubsection{Optimization of Parameters}

The direct analysis in real time (DART) ion source was initially operated with helium gas as a reagent gas at $350{ }^{\circ} \mathrm{C}$ and a flow rate of $2 \mathrm{~L} / \mathrm{min}$ as described in the previous study performed by Lesiak et al. [31]. During the preliminary study, samples were introduced using a closed-end capillary tube after dipping into the standard solution. The fragmentor voltage in the QTOF-MS was optimized first because the voltage plays an important role to transmit ions into the mass spectrometer. The fragment voltage is applied to the exit end of capillary, which is a part of ion optics maximizing the signal, but minimizing the noise contribution [122]. At high voltage, ions may produce fragments acting as an in-source fragmentor, while ions are not efficiently delivered into the quadrupole at low voltage [123]. Therefore, it is crucial to find the optimal voltage to 
maximize the transmission of ions of interest. Different fragmentor voltages from 50 to $400 \mathrm{~V}$ were applied during optimization using the individual standard solutions of bkEBDB and $\alpha-P V P$. To ensure the consistency of results, the analysis was duplicated using three replicates each day. It is showed that ions are effectively transmitted at the voltage between 200 and $300 \mathrm{~V}$ without fragmentation possibly happening at higher than $300 \mathrm{~V}$. Considering optimal voltage for both compounds, $250 \mathrm{~V}$ is chosen in this study as shown in Figure 25.

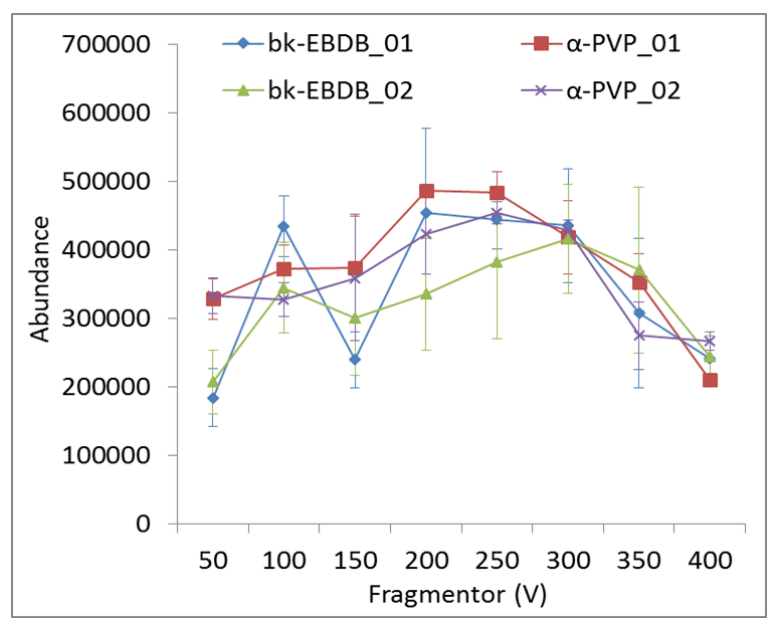

Figure 25. Optimization of the fragmentor voltage for the QTOF-MS. The ideal voltage was $250 \mathrm{~V}$ for both compounds between replicates.

Although sample introduction using the capillary tube is simple and fast, the amount of sample introduced into the mass spectrometer is not consistent. For liquid analysis, a linear rail system with 1D transmission module can be utilized to alleviate the problem with the capability of automated sample introduction. Up to 10 samples can be automatically introduced into the mass spectrometer after being delivered onto mesh spots. In the stage of optimization during experiments, each substance was analyzed in triplicate after the analysis of blank spot in the direction of analysis order as illustrated in 
Figure 26. Since instrumental responses (represented in abundance of ions) are dependent on the exposure time of each sample on the spot, the amount of delivered sample onto the mesh spot and the sample speed are important factors. To investigate the relationship between these two factors and the instrument response, different amounts of bk-MDDMA standard solution were delivered ranging from 5 to $20 \mathrm{ng}$ and analyzed at different sample speed ranging from 1.0 to $2.5 \mathrm{~mm} / \mathrm{sec}$ (Figure 28 (a)). The results showed that response from the instrument is proportional to the increased amount of sample delivered as expected. However, it is also observed that samples are not completely desorbed from the mesh when more than $15 \mathrm{ng}$ is delivered, resulting in carryover. The sample speed is related to the exposure time of each mesh spot. It is possibly explained that faster sample speed $(2.5 \mathrm{~mm} / \mathrm{sec})$ results in less exposure time at each spot. Although there was no clear relationship established between the sample speed and delivered amount of sample in terms of instrument response, it was observed that faster sample speed produces carryover between analyses. Therefore, the optimal parameters for the sample speed and sample delivery were chosen to be $1.5 \mathrm{~mm} / \mathrm{sec}$ and $10 \mathrm{ng}$, respectively.

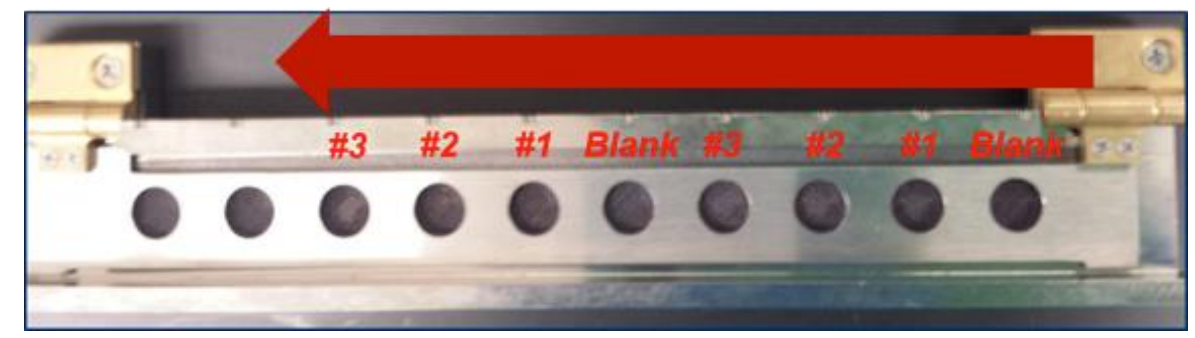

Figure 26. Sample introduction by 1D transmission module using the linear rail system. 
During the investigation of the sample speed and amount of sample delivery using the transmission module, it was noticed that samples deposited toward the end of the mesh (refer to 'back') tend to be less sensitive than those deposited toward the front of mesh (refer to 'front'). In order to prove the tendency, the same compound, $\alpha$-PVP, was delivered onto two different portions of mesh. The results showed that abundances between front and back portions were significantly different (Figure 28 (b)). It might be a result from the loss of analyte while samples in the back portion are waiting. Therefore, samples were delivered in the back portion of mesh only during the limits of detection study.

Figure 27 shows different types of insulating caps that can be utilized in DART analysis. The flat cap is normally used for the on-axis orientation of the DART source, whereas the angle cap is utilized for the $45^{\circ}$ angle configuration [100]. Although the angle cap is not commonly used for the on-axis surface or transition mode, the performance between two caps were evaluated. As presented in Figure 28 (c), the instrument response were slightly greater when the angle cap was used than the flat cap. It might be because the angle cap transports the heated reagent gas closer to the mash spot than the flat cap. However, the flat cap is used during the rest of research because abundances between two caps are not significantly different and the variation between replicates was larger when the angle cap was used than the flat one. 


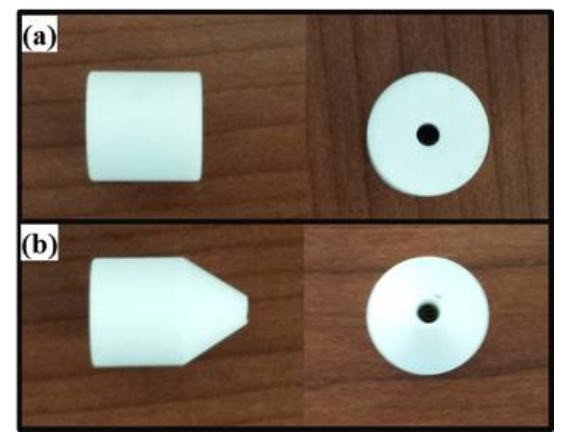

Figure 27. Different types of insulating caps: (a) flat cap and (b) angle cap.

Lastly, the distance between the DART source and the inlet of mass spectrometer was optimized to prevent the loss of analyte introducing to the mass spectrometer. It is important to have the optimal distance because the space is also where samples are introduced. As shown in Figure 28 (d), instrument response was greater when the gap is kept in $3 \mathrm{~cm}$. 


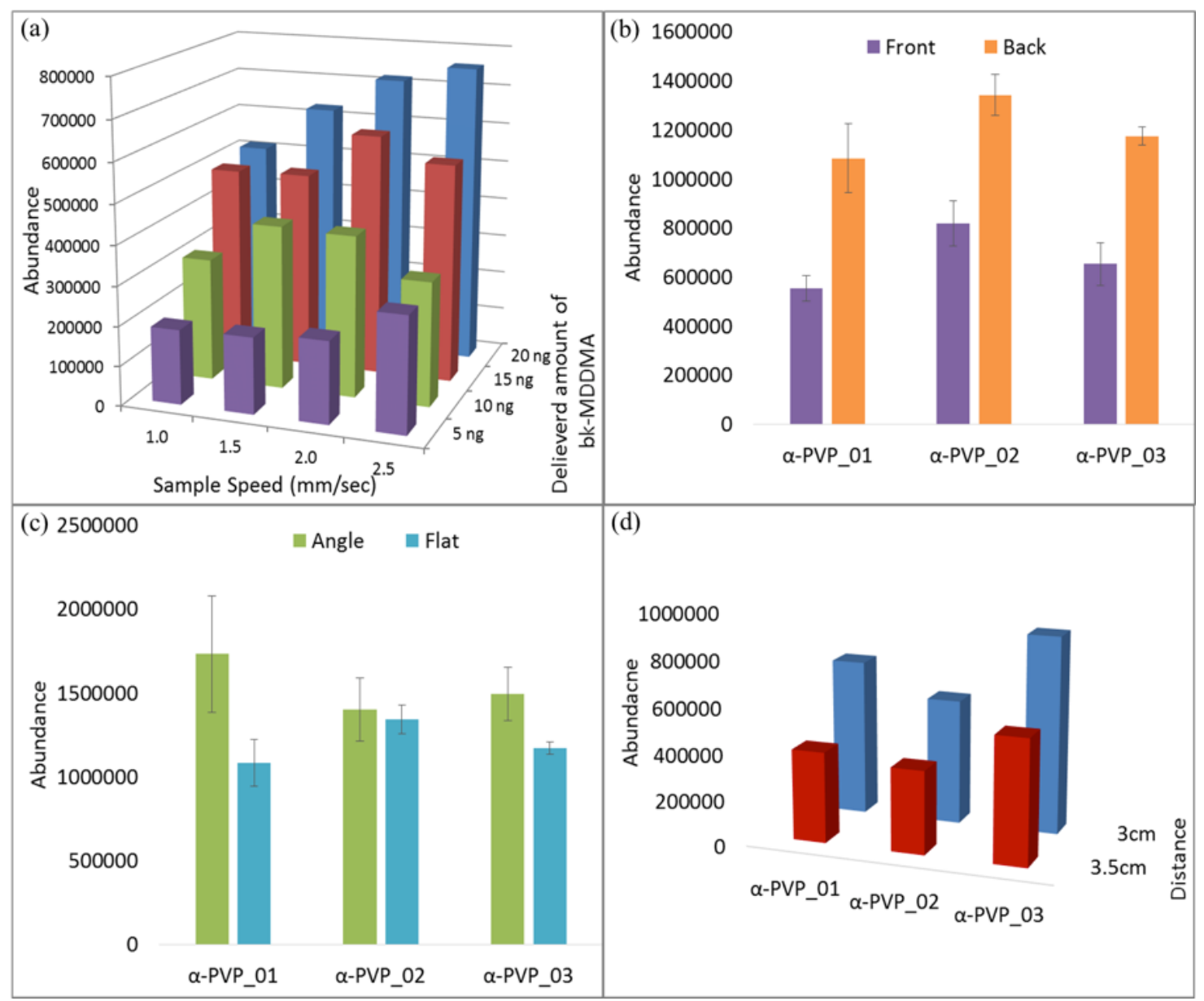

Figure 28. Optimized operating conditions for (a) the sample speed and amount of sample delivered, (b) different positions for sample delivery, (c) different types of insulating cap, and (d) distance between the DART source and the inlet of MS.

\subsubsection{Analysis of NPS standards}

The same set of NPS from the study using the Barringer IMS was investigated for the rapid identification of those compounds using DART-QTOF-MS. As previously described, the DART source is considered as a soft ionization technique producing protonated molecular ions in the positive mode. Therefore, protonated molecular ion species $\left([\mathrm{M}+\mathrm{H}]^{+}\right)$for each analyte were successfully identified in full scan mode with high mass accuracy less than $4.68 \mathrm{ppm}$ as summarized in Table 7. Mass accuracy is a 
term to describe the mass difference between the theoretical mass (exact mass) and measured mass (accurate mass) [124]. In this study, mass accuracy is expressed in parts per million (ppm).In full scan mass spectra, $[\mathrm{M}+\mathrm{H}]^{+}$is typically present as a base peak and other peaks $(\mathrm{m} / \mathrm{z}, 72.0811,135.1016,177.1481,279.1593$, and 462.1487$)$ potentially derived from the atmospheric background may be found with relatively small abundances as shown in Figure 29. These atmospheric background peaks can be excluded by acquiring air blanks before and after the sample analysis. In summation, the presence of $[\mathrm{M}+\mathrm{H}]^{+}$with high mass accuracy can facilitate the rapid screening and identification of these emerging NPS.

Table 7. List of 35 NPS analyzed by DART-QTOF-MS [119]. High mass accuracy was observed for NPS of interest $(\leq 4.68 \mathrm{ppm})$.

\begin{tabular}{|c|c|c|c|c|}
\hline NPS & $\begin{array}{c}\text { Molecular } \\
\text { Formula } \\
\end{array}$ & $\begin{array}{c}\text { Theoretical } \\
{[\mathrm{M}+\mathrm{H}]^{+}}\end{array}$ & $\begin{array}{c}\text { Measured } \\
{[\mathrm{M}+\mathrm{H}]^{+}} \\
\end{array}$ & $\begin{array}{c}\text { Mass accuracy } \\
(\mathrm{ppm})\end{array}$ \\
\hline \multicolumn{5}{|c|}{ Synthetic cathinones } \\
\hline 2,3-MDPV & $\mathrm{C}_{16} \mathrm{H}_{21} \mathrm{NO}_{3}$ & 276.1594 & 276.1594 & 0.00 \\
\hline MDPV & $\mathrm{C}_{16} \mathrm{H}_{21} \mathrm{NO}_{3}$ & 276.1594 & 276.1601 & 2.53 \\
\hline 2,3-MDMC & $\mathrm{C}_{11} \mathrm{H}_{13} \mathrm{NO}_{3}$ & 208.0968 & 208.0967 & 0.48 \\
\hline Methylone & $\mathrm{C}_{11} \mathrm{H}_{13} \mathrm{NO}_{3}$ & 208.0968 & 208.0970 & 0.96 \\
\hline 2,3-Pentylone & $\mathrm{C}_{13} \mathrm{H}_{17} \mathrm{NO}_{3}$ & 236.1281 & 236.1278 & 1.27 \\
\hline Pentylone & $\mathrm{C}_{13} \mathrm{H}_{17} \mathrm{NO}_{3}$ & 236.1281 & 236.1271 & 4.23 \\
\hline bk-DMBDB & $\mathrm{C}_{13} \mathrm{H}_{17} \mathrm{NO}_{3}$ & 236.1281 & 236.1273 & 3.39 \\
\hline bk-EBDB & $\mathrm{C}_{13} \mathrm{H}_{17} \mathrm{NO}_{3}$ & 236.1281 & 236.1270 & 4.66 \\
\hline 2-MePBP & $\mathrm{C}_{15} \mathrm{H}_{21} \mathrm{NO}$ & 232.1696 & 232.1692 & 1.72 \\
\hline 3-МePBP & $\mathrm{C}_{15} \mathrm{H}_{21} \mathrm{NO}$ & 232.1696 & 232.1691 & 2.15 \\
\hline 4-MePBP & $\mathrm{C}_{15} \mathrm{H}_{21} \mathrm{NO}$ & 232.1696 & 232.1693 & 1.29 \\
\hline$\alpha-P V P$ & $\mathrm{C}_{15} \mathrm{H}_{21} \mathrm{NO}$ & 232.1696 & 232.1692 & 1.72 \\
\hline 3-FMC & $\mathrm{C}_{10} \mathrm{H}_{12} \mathrm{FNO}$ & 182.0976 & 182.0982 & 3.29 \\
\hline 4-FMC & $\mathrm{C}_{10} \mathrm{H}_{12} \mathrm{FNO}$ & 182.0976 & 182.0979 & 1.65 \\
\hline 4-MEC & $\mathrm{C}_{12} \mathrm{H}_{17} \mathrm{NO}$ & 192.1383 & 192.1392 & 4.68 \\
\hline
\end{tabular}




\begin{tabular}{|c|c|c|c|c|}
\hline NPS & $\begin{array}{l}\text { Molecular } \\
\text { Formula }\end{array}$ & $\begin{array}{c}\text { Theoretical } \\
{[\mathrm{M}+\mathrm{H}]^{+}}\end{array}$ & $\begin{array}{c}\text { Measured } \\
{[\mathrm{M}+\mathrm{H}]^{+}}\end{array}$ & $\begin{array}{c}\text { Mass accuracy } \\
(\mathrm{ppm})\end{array}$ \\
\hline Pentedrone & $\mathrm{C}_{12} \mathrm{H}_{17} \mathrm{NO}$ & 192.1383 & 192.1389 & 3.12 \\
\hline 4-MMC & $\mathrm{C}_{11} \mathrm{H}_{15} \mathrm{NO}$ & 178.1226 & 178.1220 & 3.37 \\
\hline Buphedrone & $\mathrm{C}_{11} \mathrm{H}_{15} \mathrm{NO}$ & 178.1226 & 178.1221 & 2.81 \\
\hline 4'-MeOPPP & $\mathrm{C}_{14} \mathrm{H}_{19} \mathrm{NO}_{2}$ & 234.1489 & 234.1489 & 0.00 \\
\hline 4'-МеРHP & $\mathrm{C}_{17} \mathrm{H}_{25} \mathrm{NO}$ & 260.2009 & 260.2008 & 0.38 \\
\hline 4'-MePPP & $\mathrm{C}_{14} \mathrm{H}_{19} \mathrm{NO}$ & 218.1539 & 218.1531 & 3.67 \\
\hline bk-MDDMA & $\mathrm{C}_{12} \mathrm{H}_{15} \mathrm{NO}_{3}$ & 222.1125 & 222.1121 & 1.80 \\
\hline bk-MDEA & $\mathrm{C}_{12} \mathrm{H}_{15} \mathrm{NO}_{3}$ & 222.1125 & 222.1118 & 3.15 \\
\hline Butylone & $\mathrm{C}_{12} \mathrm{H}_{15} \mathrm{NO}_{3}$ & 222.1125 & 222.1124 & 0.45 \\
\hline Naphyrone & $\mathrm{C}_{19} \mathrm{H}_{23} \mathrm{NO}$ & 282.1852 & 282.1858 & 2.13 \\
\hline$\alpha$-Naphyrone & $\mathrm{C}_{19} \mathrm{H}_{23} \mathrm{NO}$ & 282.1852 & 282.1858 & 2.13 \\
\hline \multicolumn{5}{|c|}{ Synthetic cannabinoids } \\
\hline AM1220 & $\mathrm{C}_{26} \mathrm{H}_{26} \mathrm{~N}_{2} \mathrm{O}$ & 383.2118 & 383.2110 & 2.09 \\
\hline AM2233 & $\mathrm{C}_{22} \mathrm{H}_{23} \mathrm{IN}_{2} \mathrm{O}$ & 459.0928 & 459.0924 & 0.87 \\
\hline CPE & $\mathrm{C}_{24} \mathrm{H}_{28} \mathrm{~N}_{2} \mathrm{O}_{2}$ & 377.2224 & 377.2210 & 3.71 \\
\hline JWH-018 & $\mathrm{C}_{24} \mathrm{H}_{23} \mathrm{NO}$ & 342.1852 & 342.1854 & 0.58 \\
\hline APICA & $\mathrm{C}_{24} \mathrm{H}_{32} \mathrm{~N}_{2} \mathrm{O}$ & 365.2587 & 365.2577 & 2.74 \\
\hline MAM2201 & $\mathrm{C}_{25} \mathrm{H}_{24} \mathrm{FNO}$ & 374.1915 & 374.1902 & 3.47 \\
\hline UR-144 & $\mathrm{C}_{21} \mathrm{H}_{29} \mathrm{NO}$ & 312.2322 & 312.2315 & 2.24 \\
\hline XLR-11 & $\mathrm{C}_{21} \mathrm{H}_{28} \mathrm{FNO}$ & 330.2228 & 330.2216 & 3.63 \\
\hline \multicolumn{5}{|l|}{ Phenethylamines } \\
\hline 25I-NBOMe & $\mathrm{C}_{18} \mathrm{H}_{22} \mathrm{INO}_{3}$ & 428.0717 & 428.0714 & 0.70 \\
\hline
\end{tabular}




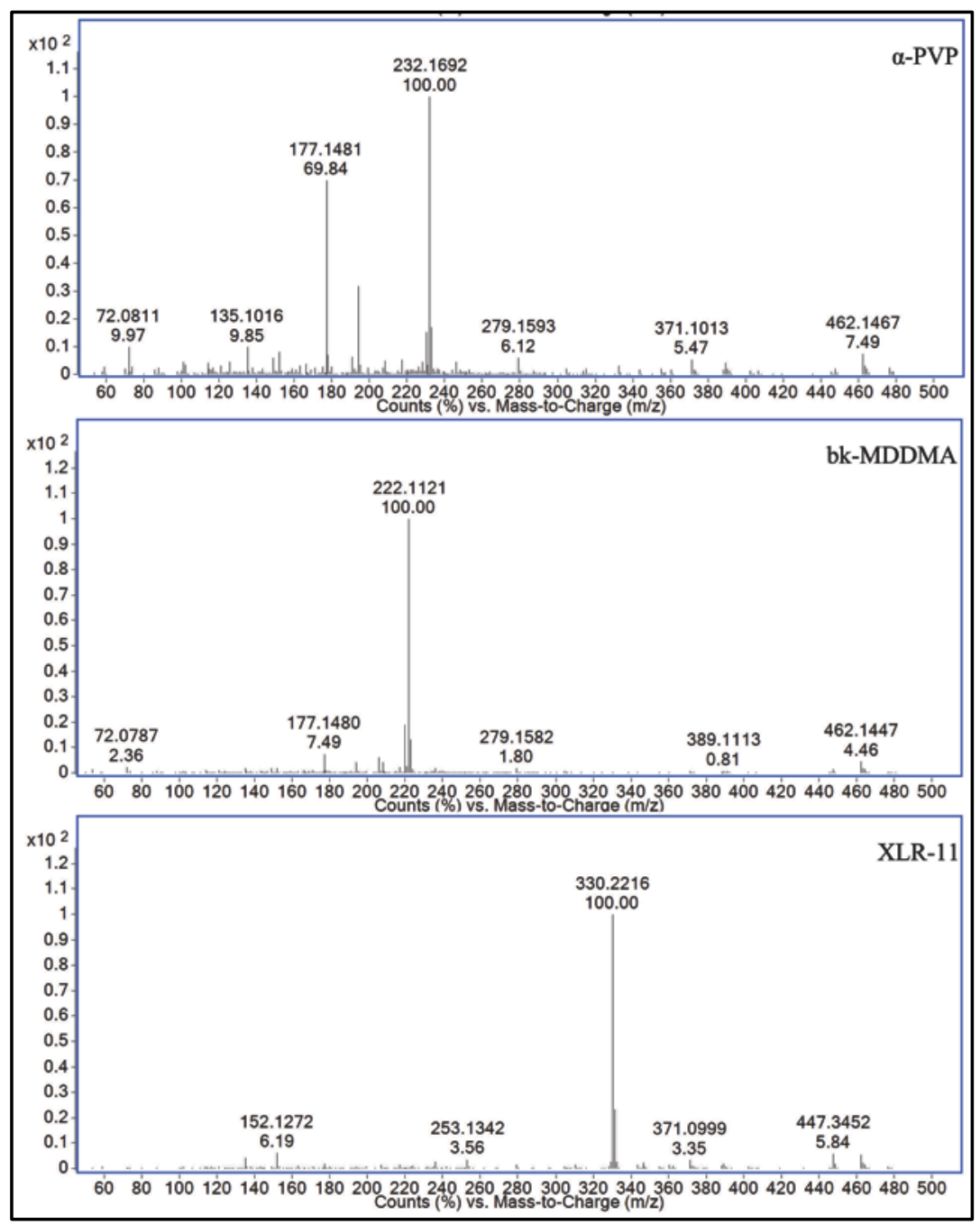

Figure 29. Full scan MS obtained using DART-QTOF-MS, (a) $\alpha-P V P$, (b) bk-MDDMA, and (c) XLR-11 [119]. The protonated molecular ion for each compound was observed as a base peak.

However, the differentiation of isomers is often challenging in full scan mode because isomers will present the same protonated molecular ions with the identical exact mass. In the case of constitutional isomers, it is possible to differentiate these isomers by comparing fragmentation patterns in product ion scan MS resulting from CID. Previously, the fragmentation of synthetic cannabinoids and synthetic cathinones were studied by Musah et al. and Lesiak et al. using DART-TOF-MS, varying the electrode 
voltage at the inlet cone of the instrument, so called in-source CID [30-31]. In our application of DART, the QTOF mass spectrometer was used enabling CID in the collision cell. The advantage of this QTOF-MS over TOF-MS is the simultaneous acquisition of full scan and product ion scan mass spectra during the MS/MS analysis, which may not be possible with in-source CID. In QTOF, the ions of interest are filtered and selected in quadrupole and the fragmentation happens in the collision cell where the collision energy (CE) is applied. The product ion scan mass spectra for bk-MDEA and bk-MDDMA are shown in Figure 30 for example. In the differentiation of constitutional isomers, the product ion scan mass spectra at the $\mathrm{CE}$ of $20 \mathrm{eV}$ were compared because most of precursor ions were extensively fragmented at $40 \mathrm{eV}$. For example, bk-MDEA and bk-MDDMA can be differentiated comparing product ion scan MS at CE $20 \mathrm{eV}$ with the presence of $\mathrm{m} / z 174.0913$ and 204.1014 for bk-MDEA, which correspond to the loss of $\mathrm{C}_{2} \mathrm{H}_{7} \mathrm{O}$ and $\mathrm{H}_{2} \mathrm{O}$. 


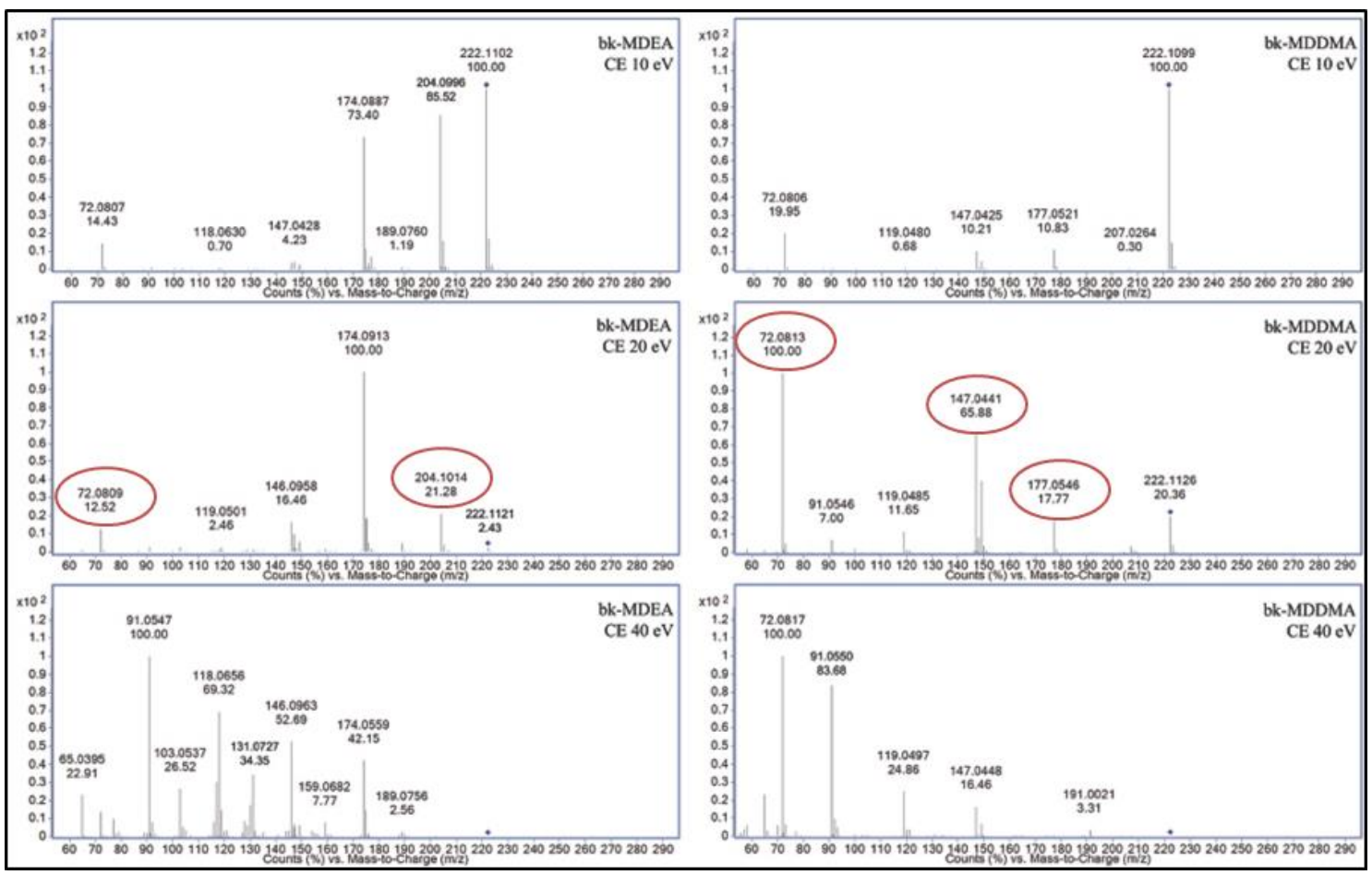

Figure 30. Product ion MS of bk-MDEA (left) and bk-MDDMA (right) at different collision energies of 10, 20, and $40 \mathrm{eV}$ [119]. Two constitutional isomers showed different fragmentation at $\mathrm{CE}$ of $20 \mathrm{eV}$.

The presence of these characteristic peaks in each product ion scan spectrum may also facilitate the qualitative analysis of isomer mixtures. As shown in Figure 31, the characteristic peaks, $m / z 204.1008$ and 177.0529 for bk-MDEA and bk-MDDMA, respectively, are present with the significant relative intensity. In addition, there were evident changes in the relative peak intensity for $m / z \quad 147.0424$ and 72.0806. The identification of other product ion peaks with relative intensities can be found in Appendix 2. The presence and relative intensity of product ions resulting from CID were consistent and repeatable in our investigation. The fragmentation of other NPS are also presented in Appendix 2. 


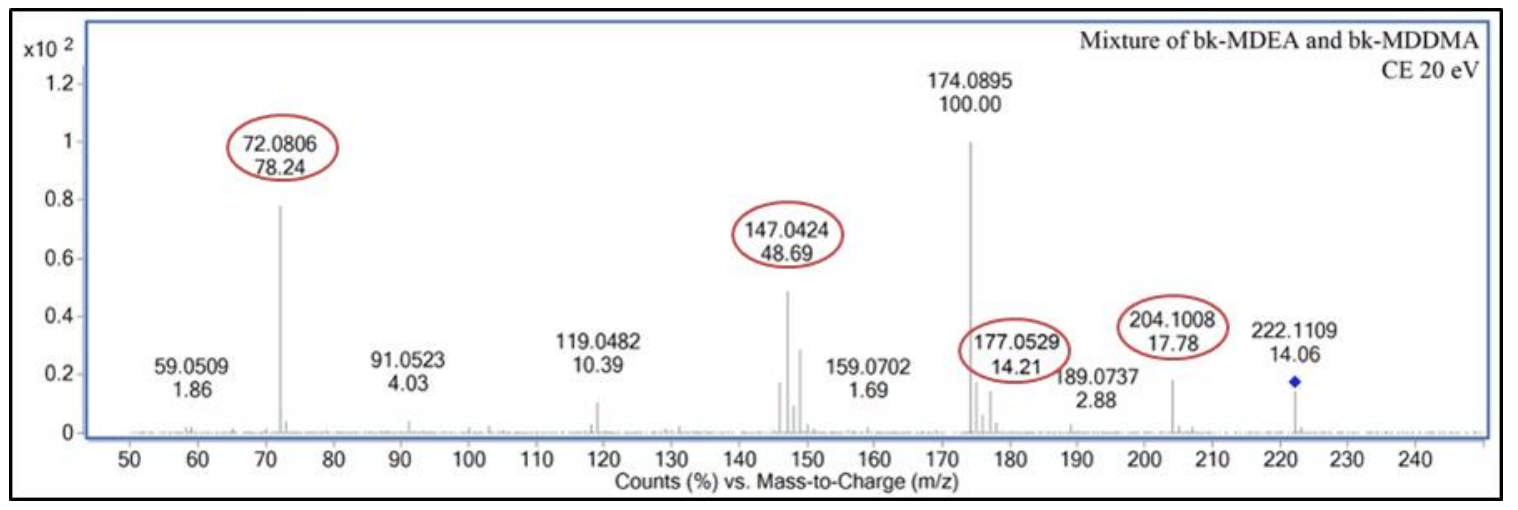

Figure 31. Product ion MS of a bk-MDEA and bk-MDDMA mixture at CE of $20 \mathrm{eV}$. Characteristic peaks from each compound were identified in the mixture analysis.

Each product ion scan MS from individual compounds was also compared to the library constructed by the same instrument, but equipped with an ESI source. The relative intensities of each peak in the mass spectra at the different $\mathrm{CE}$ for the same compounds were very similar to each other, demonstrating the benefit of the ESI mass spectral library. In addition, the fragmentation pathway for various synthetic cathinones was extensively elaborated in previous studies by other groups using LC-QTOF-MS [96, 125]. In the comparison between DART-QTOF and LC-QTOF product ion mass spectra at different collision energies for methylone, butylone, pentylone, MDPV, 4'-MePPP, bkMDEA, bk-MDDMA, and naphyrone were compared, and the presence and the relative intensity of each fragment peak were virtually identical. However, product ion mass spectra at $\mathrm{CE}$ of $10 \mathrm{eV}$ for butylone and $20 \mathrm{eV}$ for MDPV and naphyrone showed slightly different relative intensities for some fragment peaks. Although there was a minor discrepancy in the relative abundance between our results and previous studies, DARTQTOF-MS is still a valuable alternative technique for the rapid qualitative analysis of NPS with the capability of simultaneous acquisition of full scan and product ion scan mass spectra as well as fast analysis time less than two minutes. 


\subsubsection{Determination of Detection Limits}

As described in 4.1.2, the same approach was applied to determine the limits of detection for bk-EBDB, bk-MDDMA, and APICA utilizing this DART-QTOF-MS. The calibration curves were constructed on the basis of the calibration solutions ranging from 0.05 to $3.0 \mu \mathrm{g} / \mathrm{mL}$ versus instrumental responses for each calibrator. The response from the instrument refers to abundance, determined by integrating the peak area of extracted protonated molecular ion. The relationship between the amount of sample delivered and abundance showed a good linearity $\left(0.9913 \geq \mathrm{R}^{2} \geq 0.9417\right)$ in the linear range of 50 to 1500 pg (Figure 32). The calculated LODs for bk-EBDB, bk-MDDMA, and APICA by DART-QTOF-MS were ranging from 300 to $340 \mathrm{ng}$. Although the determined detection limits of DART-QTOF-MS were approximately 10 times higher than the Barringer IMS, this DART-QTOF-MS instrument provides better dynamic range than the IMS with the capability of identification for compound. 


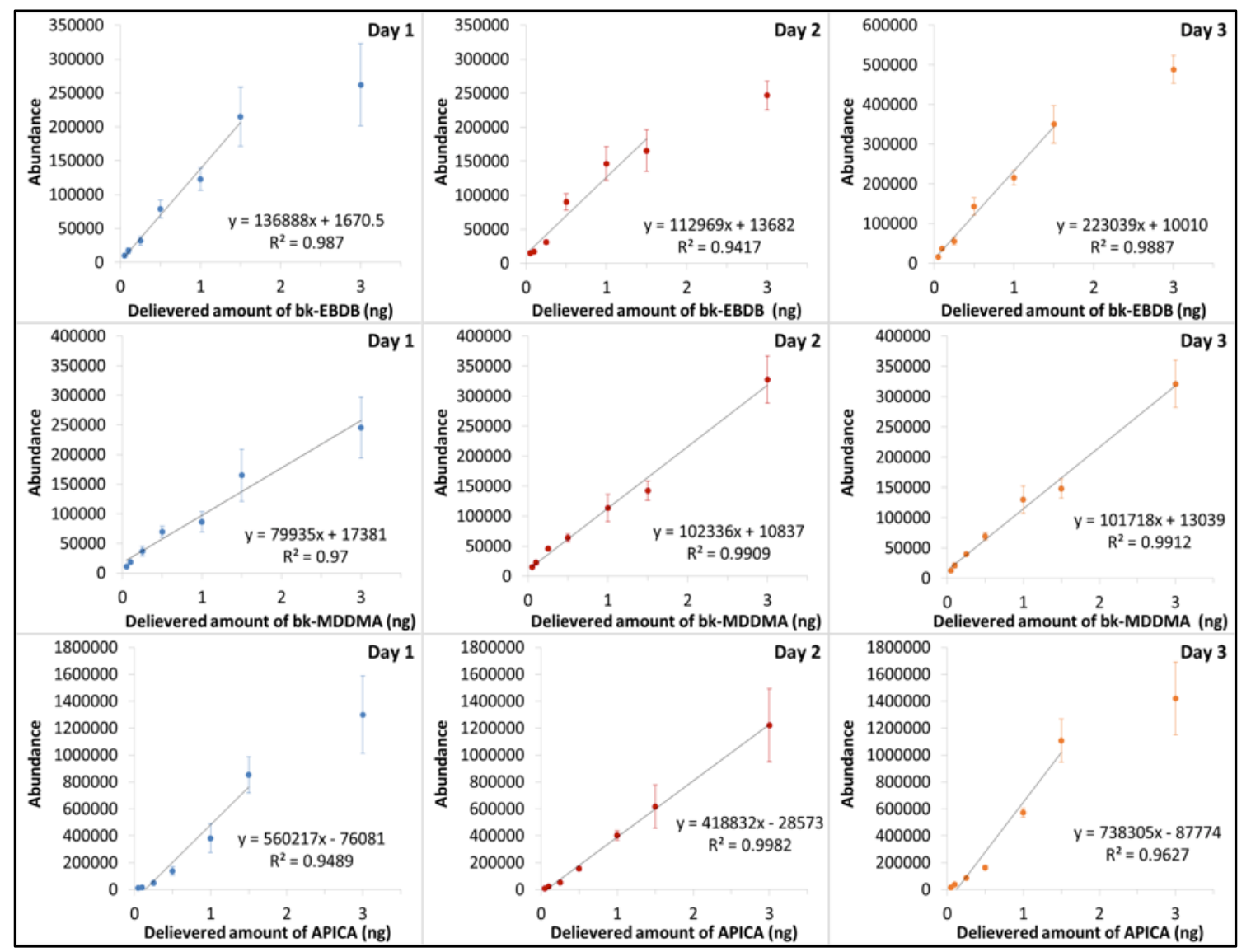

Figure 32. Calibration curves for bk-EBDB (top), bk-MDDMA (middle), and APICA (bottom) in three different days by DART-QTOF-MS.

\subsubsection{Analysis of Seized Samples}

As a continuation to the study from the analysis of seized drug samples using the Barringer IMS, the same set of seized samples prepared from 4.1.3 was analyzed using DART-QTOF-MS. Each sample was evaluated in positive mode with both full scan and product ion scan. The identification of each sample was determined after product ion scan mass spectra from the seized samples were searched using the database constructed by the ESI-QTOF. The results of product ion scan spectra at different CE are shown in

Figure 33. On the basis of library search results, blind samples 1 and 2 were identified as 
the same single compound, $\alpha$-PVP, while blind samples 3 and 4 were identified as bkMDEA and butylone, respectively. The results from the DART analysis were in agreement with the previous screening test results by the Barringer IMS and also the results from the local forensic laboratory. In the case of blind sample 4, the alarm was obtained for two compounds (butylone and bk-MDEA) from the ${ }^{63} \mathrm{Ni}$-IMS results although there was only one peak present in the ion mobility spectrum. According to the product ion scan spectrum obtained at $\mathrm{CE}$ of $40 \mathrm{eV}$ during the identification study by DART-QTOF-MS, it is found that blind sample 4 was butylone addressing the false positive alarm that occurred for bk-MDEA. It is important to note that some compounds are differentiated more effectively at CE of $40 \mathrm{eV}$ than $20 \mathrm{eV}$. Nevertheless, both results from the DART analysis and the local forensic laboratory were also agreed each other for blind sample 4. On the other hand, blind sample 3 showed some challenges in the analysis of mixture using the DART-QTOF-MS. The result from the analysis of blind sample 3 revealed that the identification of this sample was found to be bk-MDEA according to the library search. Unfortunately, the result was only partially correct compared to the screening test result obtained by the Barringer IMS and the forensic laboratory result. There are two possible reasons for the discrepancy; the use of auto MS/MS mode and the lack of separation capabilities in this DART technique. While the latter is inevitable, a different MS/MS mode can be used to alleviate the analysis of mixture. In auto MS/MS mode, the most abundant ion peak (base peak) is automatically selected for the precursor ion. In blind sample 3, bk-MEDA might have been dominant compared to another compound possibly contained. Therefore, targeted MS/MS mode was utilized to pre-select the ion of interest in an acquisition method. As shown in Figure 
33, the selection was made for $\mathrm{m} / z 232.1672$ and it was found that the compound was identified as $\alpha$-PVP according to the library search, which provided a positive result compared to the forensic laboratory result.

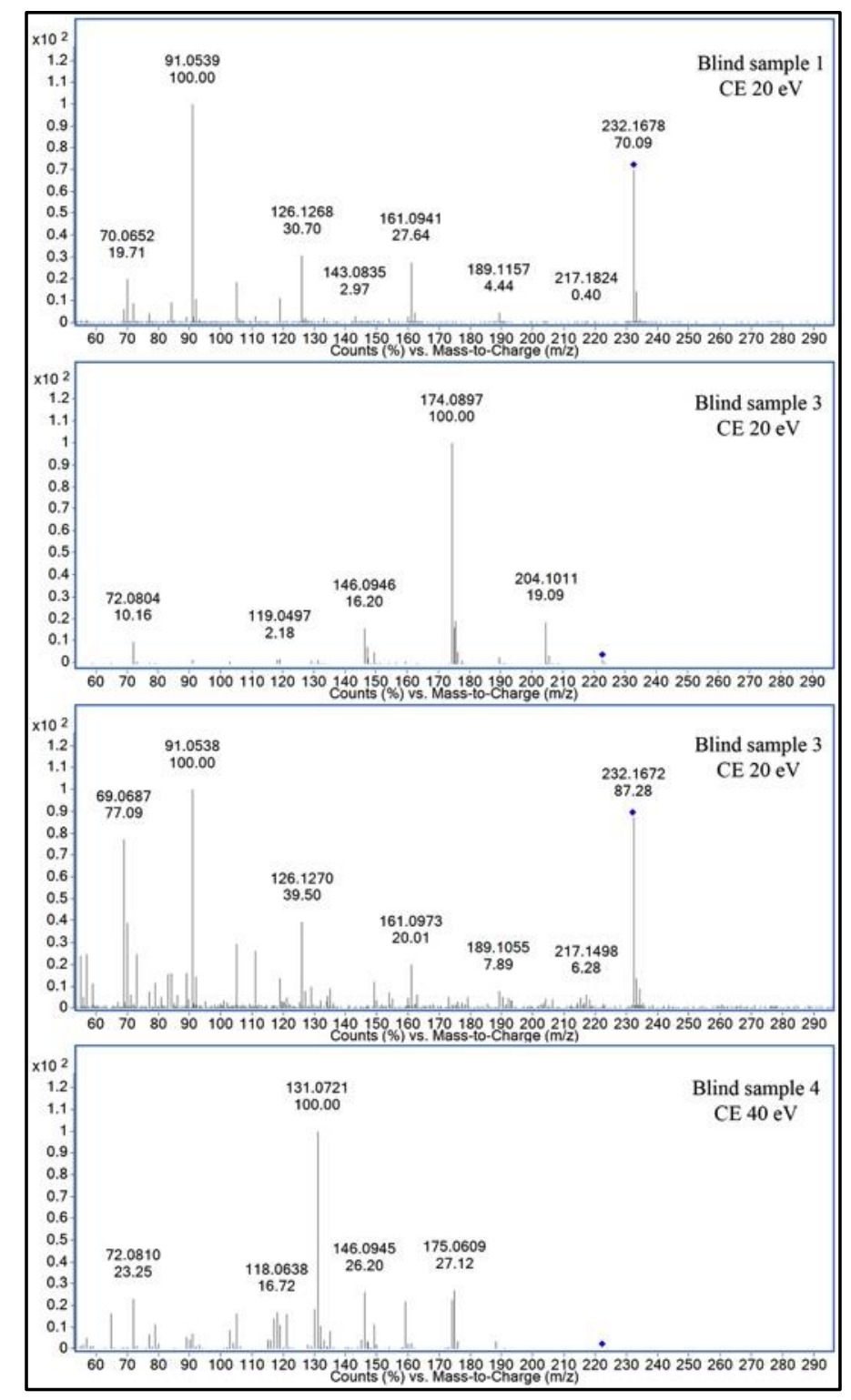

Figure 33. Product ion scan mass spectra of seized drug samples at different collision energies [119]. 


\subsubsection{Conclusions}

The novelty of DART-QTOF-MS is the rapid identification of compounds with total analysis time in less than two minutes per sample. The capability of soft ionization in the DART source allows for the presence of the protonated molecular ion. It is the greatest advantage of DART-QTOF-MS to identify molecular ions as an alternative screening technique compared to the Barringer IMS while both techniques can provide sub-nanogram detection capabilities. The coupling of DART with QTOF-MS also provides profiles of produced ions with high mass accuracy, which may facilitate the identification of unknown compounds in the purpose of seized drug analysis. In addition, it was found that product ion scan spectra between the DART ion source and ESI source produces no significant differences, allowing for the use of spectral libraries generated by ESI-QTOF. It has been shown that these libraries can be used to identify unknown compounds using product ion scan mass spectra. Moreover, the utilization of the QTOF

mass spectrometer is beneficial for the differentiation of constitutional isomers with distinctive fragmentation patterns in product ion scan mass spectra between them. Therefore, DART-QTOF-MS can facilitate the analysis of these emerging NPS by providing the capabilities of minimal to no sample preparation and rapid identification as a promising alternative screening tool. The utilization of DART-QTOF-MS for the analysis of NPS has been published in peer-reviewed literature [119]. 


\section{CHAPTER 5. DEVELOPMENT OF CONFIRMATORY METHODS FOR NPS}

\subsection{Gas Chromatography Tandem Mass Spectrometry}

As the gold standard analytical technique, GC-MS is commonly used for the identification of unknown drugs with its high sensitivity, and capability to isolate compounds of interest from complex mixtures [126]. However, the analysis of drugs using traditional GC-MS with the EI source often results in equivocal identification of compounds that are structurally close to each other. The ambiguous identification of compounds is mainly because EI mass spectra of these compounds are nearly identical with the lack of molecular ions and extensive fragmentation. The application of GCMS/MS has been a potential alternative to provide the unambiguous identification of these compounds. With the aid of GC-MS/MS, the mass spectrometric differentiation of designer drug regioisomers has been successfully achieved with the implementation of EI and/or CI sources [20, 22-23, 29, 126-127]. GC-MS/MS has also been applied to the identification and quantitation of traditional drugs of abuse, especially for the presence of GHB, opioids, cocaine, and amphetamine derivatives in human hair where a method of high sensitivity is required $[88-89,128]$. In this section 5.1 , the qualitative identification of 244 NPS was performed by GC-MS/MS with EI and CI sources to investigate the potential of the instrument as an alternative confirmatory technique in seized drug analysis.

\subsubsection{Retention-Time Locking and Multiple Reaction Monitoring Mode Optimization}

Prior to the analysis of NPS, an acquisition method used in the investigation was locked to proadifen using a retention-time locking (RTL) feature integrated in the Agilent 
Chemstation software. Retention time is an important component for the qualitative identification of substances in chromatography [129]. However, there are some variables that may affect the consistency of retention times between GC systems including slight difference in length, diameter, and film thickness of column as well as small deviation in the inlet pressure and oven temperature. The goal of retention-time locking (RTL) is to achieve same retention times between different GC systems, but same working conditions [130]. While the mathematical theory of RTL is comprehensively demonstrated in the literature [131], the procedure to lock a method using the RTL is only described in this section. First, the selection of a target compound was performed considering the ease of identification in the method. It is also recommended that the elution time of the target compound should be in the middle of the chromatogram. Once the target compound was determined, five calibration runs were initiated at different inlet pressures: target pressure (nominal method pressure), $\pm 10 \%$, and $\pm 20 \%$. A plot of inlet pressures versus the corresponding retention times acquired from those five runs was used to fix the retention time of the target compound. In our study, proadifen was used as a target compound to lock the retention time at 20.765 min. The calibration curve constructed from the RTL was stored in the acquisition method and used to relock the retention time whenever column maintenance was performed. In addition, the flow rate of the carrier gas $(\mathrm{He})$ was adjusted in accordance with the optimized inlet pressure for the target compound.

The parameters for MRM mode with both EI and CI was optimized on the basis of a preliminary study of NPS in full scan mode. The optimization process consists of choosing appropriate precursor ion(s), selecting 2 to 3 transitions from the precursor ion(s) to product ion(s), and finding desirable collision energy and dwell time for each 
transition. The basic criteria for the selection of precursor ions included ions with high mass, abundance of ions, and uniqueness of ions. A molecular ion was the first choice in most cases when it is present with an acceptable relative intensity larger than $20 \%$. However, the base peak was also selected as a precursor ion to improve the sensitivity when the intensity of the molecular ion was less than $10 \%$ (for example, in EI MRM mode). Once the precursor ion is chosen, product ion mass spectra were acquired at various $\mathrm{CE}$ of $5,10,15,20,25$, and $30 \mathrm{eV}$. A series of product ion scan methods at different CE was generated using the software called 'Design Experiments Assistant' by Agilent Technologies after a template product ion scan method is created. The acquired product ion scan mass spectra were examined using the MassHunter Qualitative Analysis software in order to select the quantifier and qualifier transitions. After a temporary MRM method was established from the selected transitions, a new set of MRM methods at different $\mathrm{CE}$ from $4 \mathrm{eV}$ up to $60 \mathrm{eV}$ were generated using the Design Experiment Assistant software. The results of MRM mass spectra at various CE was subsequently evaluated using the software 'Analyze Experiment Assistant', in which the loaded data is automatically analyzed. As a result, the final MRM method was produced with ideal collision energies where the product ions are most abundant at the specific transition. Finally, the Dynamic MRM assistant software (Agilent Technologies) was used to optimize the dwell times for each transition.

\subsubsection{Qualitative analysis of 244 compounds}

A total of 244 compounds listed in Appendix 1 were successfully analyzed by GCMS/MS with EI, CI, or both in full scan and MRM mode. The list of 244 compounds 
includes 148 synthetic cannabinoids, 53 synthetic cathinones, 24 phenethylamines, 1 ketamine, 4 tryptamines, 6 piperazines, and 8 additional compounds. Of 244 compounds, the different types of 34 NPS were selected carefully as a subset in the basis of drug schedules by U.S. DEA [132]. The comparison of these 34 NPS between EI and CI in full scan mode was performed and presented as a preliminary study. Table 8 summarizes major peaks (a base peak and two other abundant peaks) from EI and CI full scan mass spectra for these 34 NPS of interest with their relative abundance. The molecular ions were present in EI full scan spectra for most of the synthetic cannabinoids (16 out of 34), whereas extensive fragmentation was observed for all of the synthetic cathinones and some of the synthetic cannabinoids (18 out of 34), which are highlighted in gray.

Table 8. Summary of major peaks in EI and CI full scan MS for the analytes of interest with the molecular ions, shown in bold. Molecular ions are absent for the highlighted analytes in EI full scan MS [133].

\begin{tabular}{|c|c|c|c|c|}
\hline Analyte & $\begin{array}{l}\text { Retention } \\
\text { Time } \\
\text { (min) }\end{array}$ & $\begin{array}{l}\text { Molecular } \\
\text { Weight } \\
\text { (amu) }\end{array}$ & $\begin{array}{l}\text { Major Peaks in EI Full Scan MS } \\
\text { (Relative Abundance, \%) }\end{array}$ & $\begin{array}{l}\text { Major Peaks in CI Full Scan MS } \\
\text { (Relative Abundance, \%) }\end{array}$ \\
\hline 3-FMC & 9.715 & 181.09 & $58(100), 95$ (12.3), $123(6.1)$ & $182(100), 164(36.0), 58(7.7)$ \\
\hline 4-MMC & 11.635 & 177.12 & $58(100), 91(8.7), 119(5.8)$ & 160 (100), 178 (72.1), 58(17.8) \\
\hline 4-MEC & 12.352 & 191.13 & $72(100), 91(8.3), 119(5.5)$ & 192 (100), 174 (69.3), 72 (14.0) \\
\hline 3,4-DMMC & 13.188 & 191.13 & $58(100), 133(5.5)$ & 174 (100), 192 (82.3), 163 (24.0) \\
\hline Methedrone & 13.685 & 193.11 & $58(100), 135(8.3), 77(6.2)$ & 176 (100), 194 (60.6), 165 (29.2) \\
\hline Methylone & 14.685 & 207.09 & $58(100), 149(6.2)$ & 190 (100), 208 (99.9), 160 (41.3) \\
\hline MDPV & 19.124 & 275.15 & $126(100), 149(5.5)$ & 276 (100), $126(55.0), 207$ (42.4) \\
\hline CP-47,497 & 22.919 & 318.26 & 215 (100), 233 (72.9), 318 (5.9) & 301 (100), 233 (39.3), 318 (5.8) \\
\hline XLR-11 & 22.976 & 329.22 & 232 (100), 144 (24.9), 329 (7.2) & 330 (100), 310 (67.6), 125 (18.6) \\
\hline CP-47,497 C8 & 23.670 & 332.27 & 215 (100), 233 (75.1), $332(6.3)$ & 315 (100), 247 (25.7), 332 (5.5) \\
\hline JWH-251 & 24.783 & 319.19 & $214(100), 144(23.5), 116(6.5)$ & 320 (100), 214 (22.9), 304 (22.1) \\
\hline JWH-203 & 25.399 & 339.14 & $214(100), 144$ (24.6), $116(7.0)$ & 340 (100), 214 (24.1), 127 (13.1) \\
\hline JWH-250 & 25.466 & 335.19 & $214(100), 144$ (24.3), $116(6.0)$ & 336 (100), $214(30.5), 320$ (18.8) \\
\hline JWH-302 & 25.732 & 335.19 & 214 (100), 144 (23.9), $116(5.9)$ & $336(100), 214(19.2), 320(5.0)$ \\
\hline RCS-4 & 25.867 & 321.17 & 135 (100), 321 (87.3), 264 (78.9) & $322(100), 188$ (34.0), 135 (21.5) \\
\hline JWH-249 & 26.065 & 383.10 & $214(100), 144(21.4), 116(5.4)$ & 384 (100), 288 (66.8), 214 (46.5) \\
\hline JWH-201 & 26.093 & 335.19 & $214(100), 144(22.5)$ & $336(100), 188(36.2), 214(27.0)$ \\
\hline AM694 & 26.596 & 435.05 & 232 (100), 435 (51.8), 220 (49.5) & 436 (100), 310 (23.7), 231 (12.3) \\
\hline JWH-073 & 26.596 & 327.16 & 200 (100), 327 (92.6), 284 (66.6) & 328 (100), $200(9.9)$ \\
\hline
\end{tabular}




\begin{tabular}{|c|c|c|c|c|}
\hline Analyte & $\begin{array}{c}\text { Retention } \\
\text { Time } \\
(\mathrm{min}) \\
\end{array}$ & $\begin{array}{c}\text { Molecular } \\
\text { Weight } \\
(\mathrm{amu}) \\
\end{array}$ & $\begin{array}{l}\text { Major Peaks in EI Full Scan MS } \\
\quad \text { (Relative Abundance, \%) }\end{array}$ & $\begin{array}{l}\text { Major Peaks in CI Full Scan MS } \\
\text { (Relative Abundance, \%) }\end{array}$ \\
\hline APINACA & 26.918 & 365.25 & 215 (100), 294 (29.8), 365 (16.3) & $135(100), 366(23.7), 214(14.1)$ \\
\hline JWH-022 & 27.144 & 339.16 & 155 (100), 127 (72.0), 339(58.5) & 340 (100), 212 (10.2) \\
\hline JWH-018 & 27.161 & 341.18 & 214 (100), 284 (83.0), 341 (75.2) & 342 (100), 214 (14.4), 155 (9.6) \\
\hline JWH-019 & 27.759 & 355.19 & 355 (100), 284 (96.1), 228(94.4) & 356 (100), 202 (20.9), 157 (8.8) \\
\hline AM2201 & 27.884 & 359.17 & 359 (100), 232 (98.7), 284 (93.7) & 360 (100), 340 (11.8), 232 (10.7) \\
\hline $\mathrm{CPE}$ & 28.008 & 376.22 & 98 (100), 70 (5.9) & 377 (100), 98 (65.2), 359 (7.3) \\
\hline JWH-122 & 28.031 & 355.19 & 355 (100), 214 (83.8), 298 (75.0) & 356 (100), 214 (14.2), 169 (8.5) \\
\hline RCS-8 & 28.256 & 375.22 & 254 (100), 144 (21.0), 55 (9.2) & 376 (100), 360 (33.6), 254 (29.2) \\
\hline JWH-398 & 28.369 & 375.14 & 214 (100), 375 (67.1), 318 (63.4) & $376(100), 214(16.5), 189(8.4)$ \\
\hline AM2233 & 28.409 & 458.09 & $98(100), 70(6.1)$ & 459 (100), 98 (65.0), $331(8.2)$ \\
\hline CB-13 & 28.420 & 368.18 & 171 (100), 368 (47.7), 297 (44.8) & 369 (100), 241 (10.7), 155 (8.3) \\
\hline APICA & 28.437 & 364.25 & 214 (100), 307 (28.3), 364 (21.7) & 365 (100), 135 (43.4), 213 (22.3) \\
\hline JWH-081 & 29.104 & 371.19 & 371 (100), 214 (71.1), 314 (65.0) & 372 (100), 188 (34.5), 355 (17.6) \\
\hline AM1220 & 30.018 & 382.20 & 98 (100), 70 (6.3) & 383 (100), 98 (96.6), 286 (14.5) \\
\hline JWH-200 & 30.425 & 384.18 & $100(100), 127$ (5.9), $56(5.5)$ & 385 (100), 100 (15.0), $281(10.3)$ \\
\hline
\end{tabular}

Figure 34 shows EI full scan mass spectra for some of the phenylacetylindoles, including JWH-251, JWH-250, and JWH-249. The common fragment ions at $\mathrm{m} / \mathrm{z}$.214, 144, and 116 were observed with significant relative abundance, but extremely low relative intensity for a molecular ion $(<1 \%)$. The EI mass spectra of JWH-250 regioisomers, JWH-201 and JWH-302, were virtually identical as Figure 34 with the lack of molecular information. The low abundance of molecular ions is inconsistent with the study reported by Uchiyama et al. where they reported the presence of the molecular ion for JWH-250 and JWH-251 with a relative intensity of 5\% [134]. 


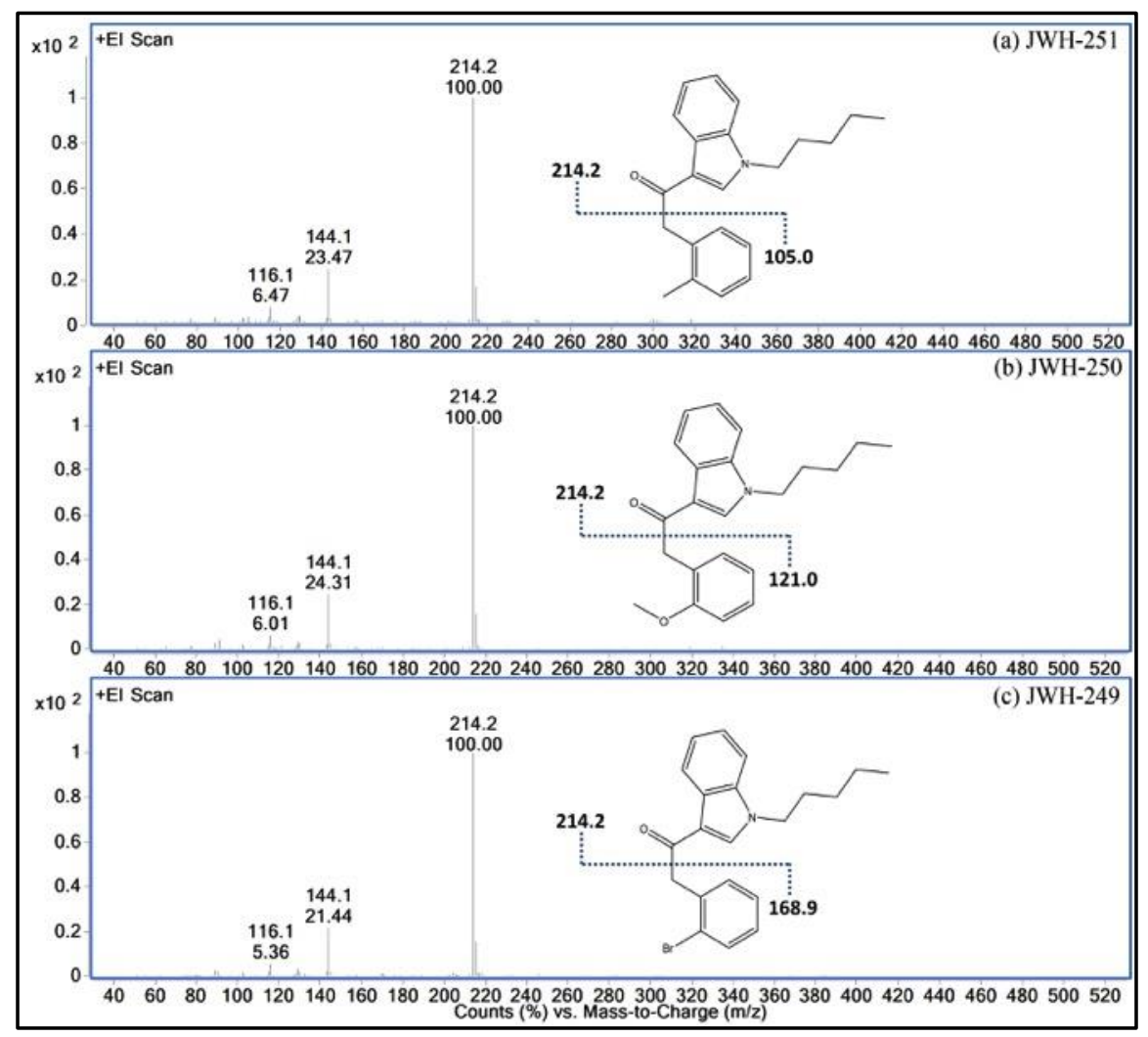

Figure 34. EI full scan mass spectra for (a) JWH-251, (b) JWH-250, and (c) JWH-249 [133]. Unambiguous identification of these compound is challenging in EI full scan mode because of the mass spectral similarity.

Other kinds of cannabimimetic indoles, such as CPE, AM2233, and AM1220, also demonstrated the same issue as shown in Figure 35. EI full scan mass spectra for these compounds are similar to each other with the presence of common fragment ions at $\mathrm{m} / \mathrm{z}$ 98 and 70. Molecular ions were barely observed in the mass spectra for each compound in our investigation, whereas a trace of molecular ions was seen in EI mass spectra of AM-1220 and AM-2233 reported by the other study [135]. The identification of these substances that are extensively fragmented with the EI source is often challenging because of the similar mass spectra with the absence or relatively low abundance of the molecular ion. 


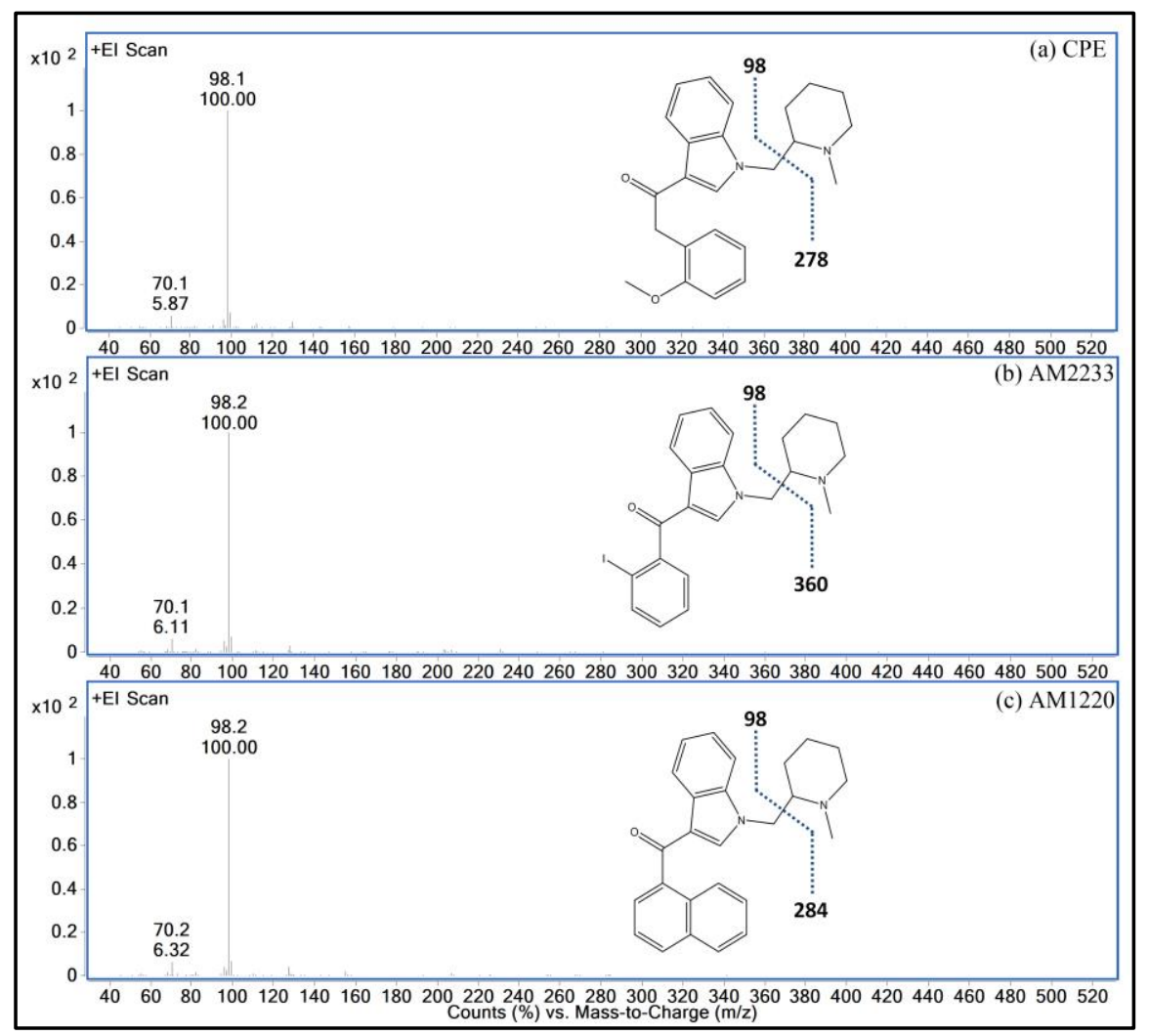

Figure 35. EI full scan mass spectra for (a) CPE, (b) AM2233, and (c) AM1220 [133]. Similar mass spectra were observed for these compounds in EI full scan mode.

For those substances that are easily fragmented in EI, the implementation of the CI source will be beneficial because protonated molecular ions are normally present in CI mass spectra with its softer ionization capability as mentioned previously in 2.3.3.1. Table 8 shows the summary of major peaks from CI full scan mass spectra for each substance with protonated molecular ions as the most abundant peak, except CP 47, 497, and its C8 homolog. The actual CI full scan mass spectra of JWH-251, JWH-250, and JWH-249 are shown in Figure 36 with molecular information of each substance. It is expected that the collection of CI mass spectra can be used for a supplemental library database in the identification of unknown compounds. As a result, the identification of 
NPS can be facilitated with the presence of protonated molecular ions in the CI mass spectra.

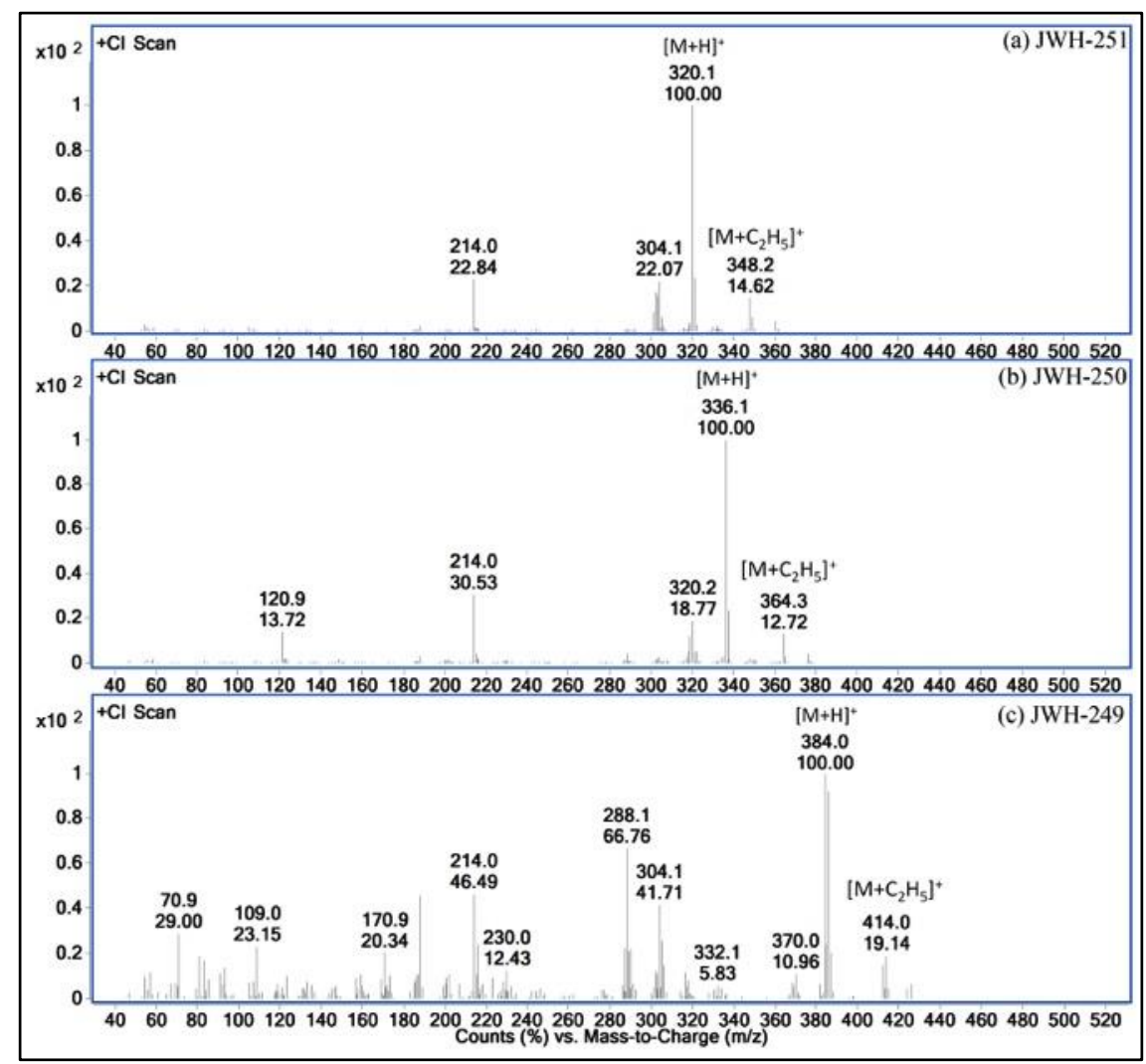

Figure 36. CI full scan mass spectra for (a) JWH-251, (b) JWH-250, and (c) JWH-249 [133]. The protonated molecular ion from each compound were observed with the characteristic ethyl adduct in CI full scan mode.

Highly selective analysis of these NPS was also achieved by MRM mode using a GC-MS/MS instrument. The presence of isomers and the similar retention times for some compound may cause the inaccurate identification of compounds and co-elution in the analysis of mixtures. However, with the implementation of MRM mode, some of the co-eluted compounds can be separated by extracting chromatograms for specific transitions from the co-eluted peak. In order to isolate the specific analyte of interest, it is 
necessary for these analytes to have characteristic transitions that can help differentiate them from others. The extensive fragmentation in the EI source may result in the generation of the same transitions with the lack of molecular information. Therefore, the EI MRM method may not be adequate to separate the co-elution. Yet, the CI MRM method is more useful because it provides characteristic transitions from the molecular ion in terms of specificity. Optimized MRM transitions for 34 NPS of interest utilizing the EI and CI sources are shown in Appendix 3 and Appendix 4. The most commonly encountered problem in the characterization of NPS is the presence of regioisomers, for example, 2-FMC, 3-FMC, and 4-FMC. A previous study has shown the differentiation of regioisomers, fluorocathinones and fluoroamphetamine, with the implementation of the CI source and product ion scan mass spectrometry [20, 29]. In our investigation, regioisomeric phenylacetylindoles with the methoxy group at ortho-, metha-, and parapositions (JWH-250, JWH-302, and JWH-201) were evaluated. With the application of the CI MRM method, it was possible to differentiate these regioisomers with the presence of characteristic transitions at different relative abundances on top of the common product ion at $m / z, 121$ as shown in Figure 37. 


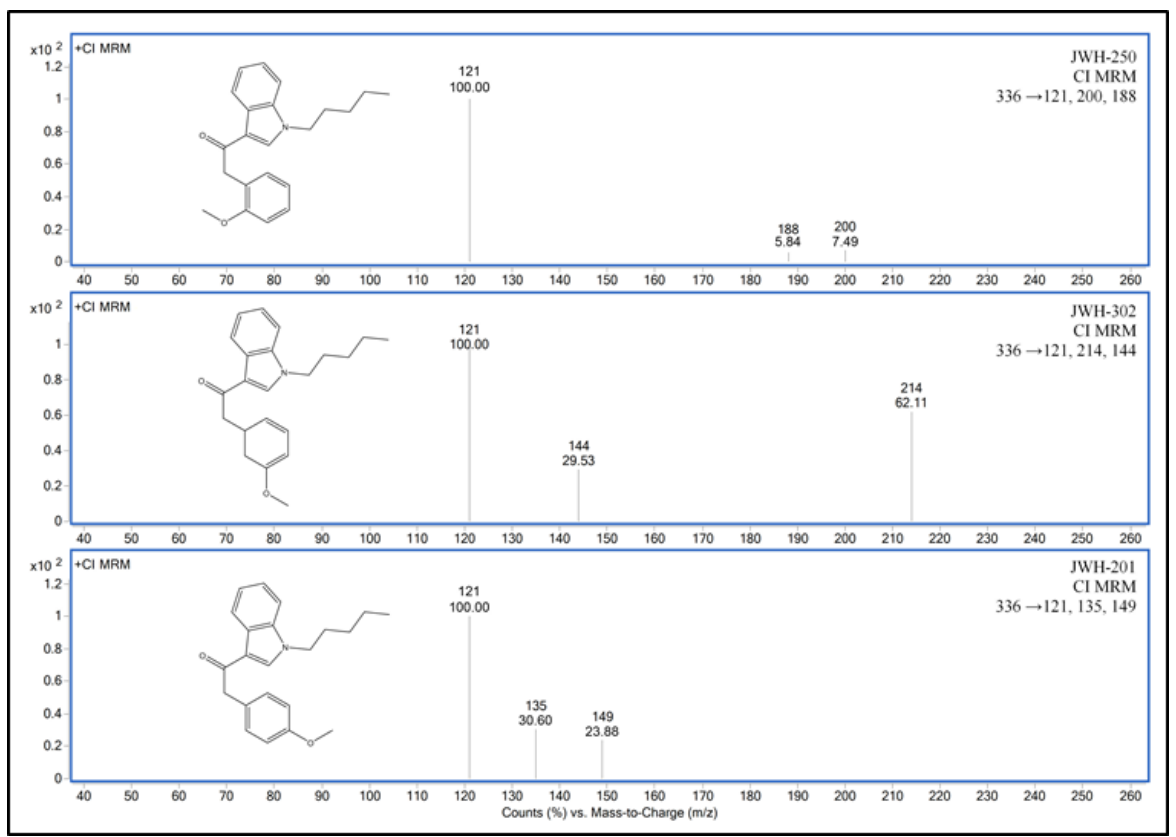

Figure 37. CI MRM mass spectra for the regioisomers of synthetic cannabinoids: JWH-250, JWH-302, and JWH-201 [133].

\subsubsection{Determination of Detection Limits}

The developed MRM method was validated by determining the limits of detection (LODs) for seven compounds. The calibration solutions were prepared in methanol at various concentrations ranging from 50 to $1200 \mathrm{ng} / \mathrm{mL}$ by serial dilution. A couple of parameters were re-optimized to achieve the best performance during the LOD study with both EI and CI sources, including a gain factor of MS increased from 1 to 50, a flow rate of helium quench gas at $2.0 \mathrm{~mL} / \mathrm{min}$, and the pulsed splitless injection mode. Each calibration solution was analyzed in 3 replicate injections in order to ensure the reliability of the qualitative analytical method for those NPS. The criteria for each calibrator was a signal-to-noise ratio $(\mathrm{S} / \mathrm{N})$ of three. The calibration curves were subsequently constructed in the different linear ranges using Agilent MassHunter Quantitative Analysis as shown in Figure 38. The results from other compounds are shown and compared 
between the EI and CI sources in Table 9. The calibration curves for each compound showed a good correlation coefficient of linearity $\left(\mathrm{R}^{2} \geq 0.99\right)$.
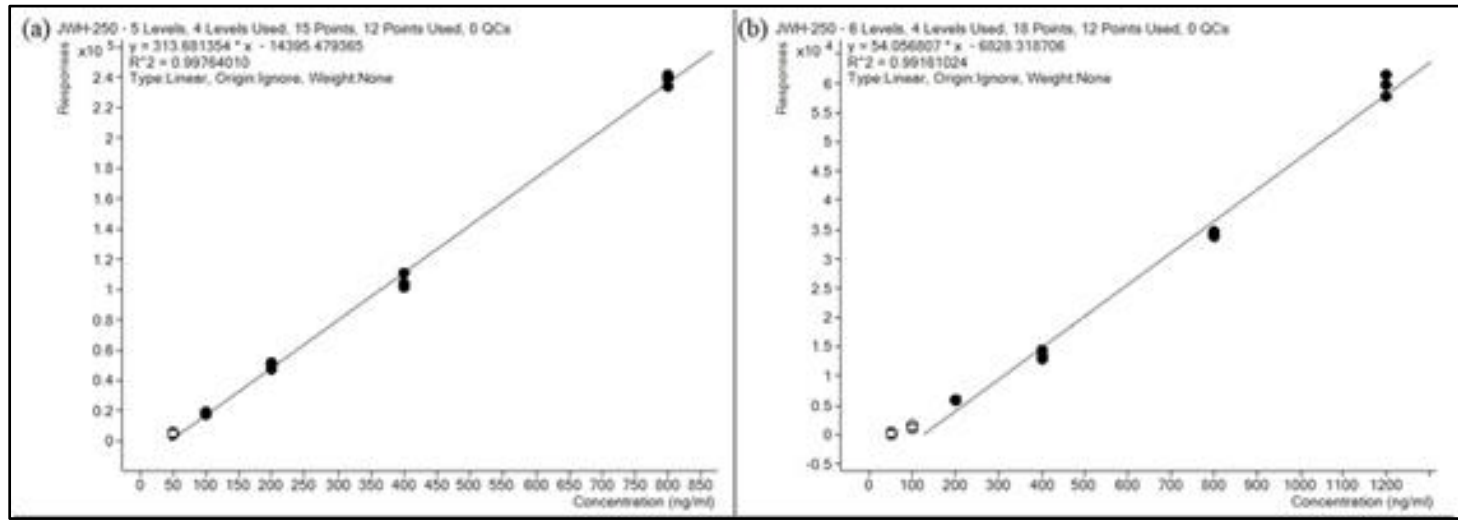

Figure 38. Four-point calibration curves for JWH-250 from GC-MS/MS with (a) EI and (b) CI using the software MassHunter Quantitative Analysis. White points were excluded from constructing calibration curves because of the disqualification for $\mathrm{S} / \mathrm{N}$ of three.

The LODs were calculated on the basis of the standard error and slope of linear correlation for each compound as previously discussed in 4.1.2. The comparison of LOD between EI and CI demonstrated that EI-MS/MS is more sensitive than CI-MS/MS according to our results shown in Table 9. Nevertheless, the CI MRM method can play an important role in providing soft identification of these emerging NPS with the presence of molecular ions and its analogs as a qualitative analysis tool when a sufficient amount of sample is available. Therefore, the implementation of the CI source as well as MRM mode with both EI and CI may facilitate the unambiguous identification of seized designer drugs and their analogs by providing additional spectral library database. 
Table 9. Limits of detection (LODs) comparison between EI and CI for representative compounds [133].

\begin{tabular}{ccccc|cccc}
\hline & \multicolumn{5}{c|}{ EI } & \multicolumn{5}{c}{ CI } \\
\cline { 2 - 9 } NPS & $\begin{array}{c}\text { Linear } \\
\text { range } \\
(\mathrm{ng} / \mathrm{mL})\end{array}$ & Slope & $\mathrm{R}^{2}$ & $\begin{array}{c}\text { LOD } \\
(\mathrm{ng} / \mathrm{mL})\end{array}$ & $\begin{array}{c}\text { Linear } \\
\text { range } \\
(\mathrm{ng} / \mathrm{mL})\end{array}$ & Slope & $\mathrm{R}^{2}$ & $\begin{array}{c}\text { LOD } \\
(\mathrm{ng} / \mathrm{mL})\end{array}$ \\
\hline \hline 4-MMC & $100-800$ & 123.88 & 0.999 & 37.5 & $100-1200$ & 39.71 & 0.993 & 123.5 \\
MDPV & $100-800$ & 397.57 & 0.997 & 63.5 & $200-1200$ & 14.84 & 0.990 & 162.9 \\
CP-47,497 & $50-800$ & 57.96 & 0.995 & 19.9 & $200-1200$ & 25.33 & 0.990 & 147.8 \\
CP-47,497 C8 & $50-800$ & 102.38 & 0.997 & 43.2 & $200-1200$ & 13.95 & 0.991 & 149.4 \\
JWH-250 & $100-800$ & 313.68 & 0.998 & 42.9 & $200-1200$ & 54.06 & 0.992 & 135.5 \\
JWH-302 & $100-800$ & 104.53 & 0.997 & 49.9 & $200-1200$ & 10.84 & 0.995 & 91.1 \\
AM694 & $100-800$ & 22.30 & 0.996 & 68.8 & $200-1200$ & 4.81 & 0.993 & 106.2 \\
\hline
\end{tabular}

\subsubsection{Analysis of Seized Samples}

A total number of 9 seized drug samples extracted with methanol were provided by a local forensic laboratory in 2013. The identity and concentration of each sample was not provided at the time of receipt for the purpose of blind testing. The qualitative analysis of these samples was performed using GC-MS/MS in full scan mode with EI and $\mathrm{CI}$ as well as in MRM mode with CI after further dilution with methanol $(1: 1000, v / v)$ to prevent overloading of samples in the GC system. It was found that all seized samples contained a single compound, which was identified as methylone with the retention time at $14.722 \pm 0.003 \mathrm{~min}$ for $\mathrm{CI}$ and $14.717 \pm 0.007 \mathrm{~min}$ for EI. Although the average retention time from these samples were slightly discrepant with the results from the analysis of the standard solution (Table 8), it is still within the fair range of variation, $0.25 \%$ for CI and $0.22 \%$ for EI, similar to previous reports by Giarrocco et al. [129]. One example of acquired mass spectra from the seized samples is shown in Figure 39. 
As previously discussed, the main disadvantage of the EI source is extensive fragmentation for some compounds. It is assumed that the seized sample falls into the synthetic cathinone or phenethylamine groups with the base peak commonly observed as a result of the formation of the immonium ion, $\mathrm{m} / \mathrm{z}$ 58. Further investigation was performed to identify the seized sample by CI full scan and MRM mode. The acquired CI full scan spectrum enabled the unambiguous identification of the compound with the presence of the protonated molecular ion $(\mathrm{m} / \mathrm{z} 208)$ as well as the byproduct species, $\left[\mathrm{M}+\mathrm{C}_{2} \mathrm{H}_{5}\right]^{+}$and $\left[\mathrm{M}+\mathrm{C}_{3} \mathrm{H}_{5}\right]^{+}$, corresponding to $\mathrm{m} / z, 236$ and 248 , respectively.

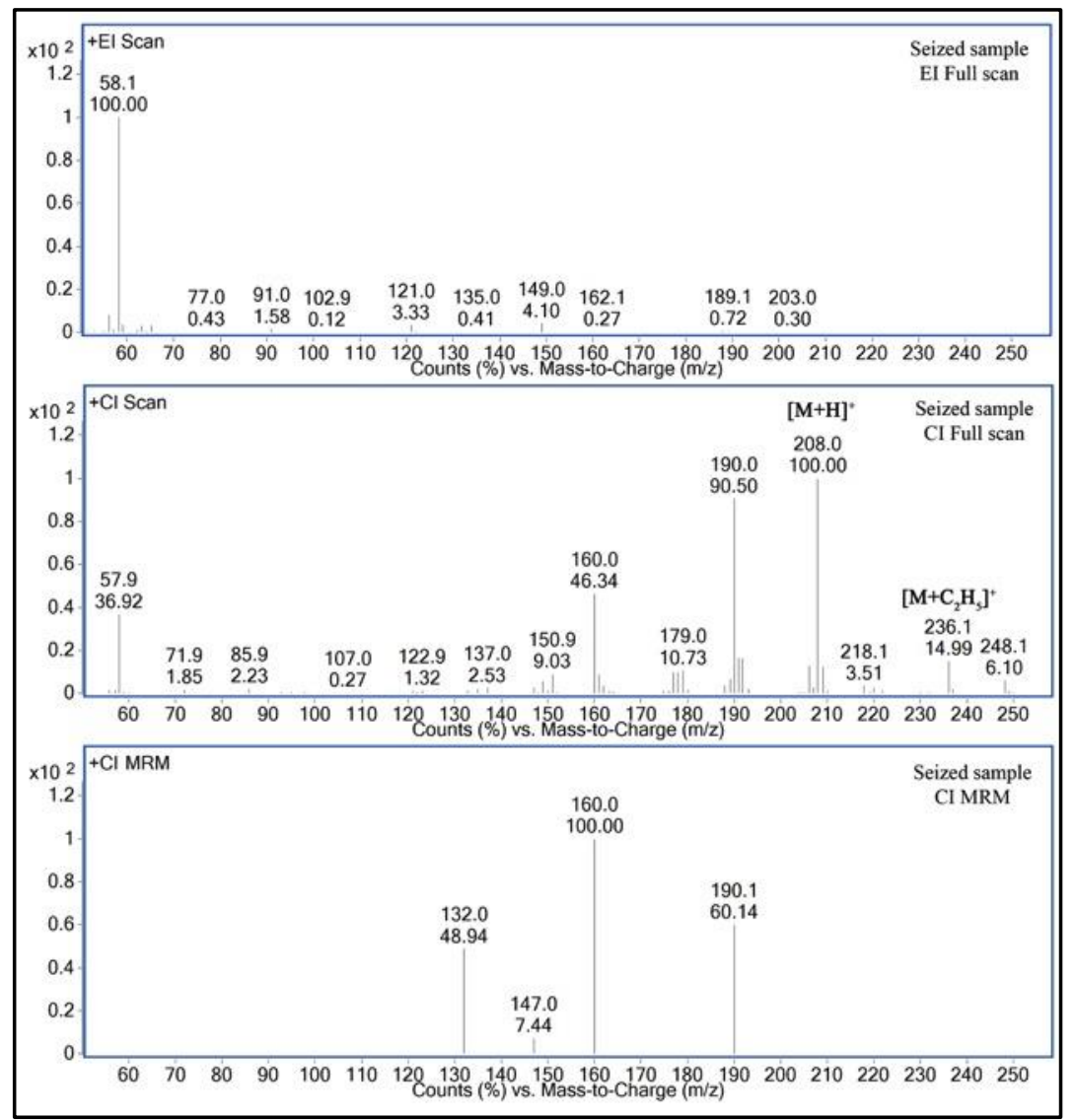

Figure 39. Mass spectra of seized sample by EI full scan (top), CI full scan (middle), and CI MRM (bottom). 
Further confirmation of the seized sample identity was performed with the CI MRM mass spectrum providing additional information as shown in Figure 39. Each mass spectrum acquired from the seized sample was also compared to mass spectra obtained from the standard solution as shown in Figure 40. Although there were variations in the relative intensity of peaks in CI full scan and MRM mass spectra, those variations were not significant enough for the identification of the seized sample.

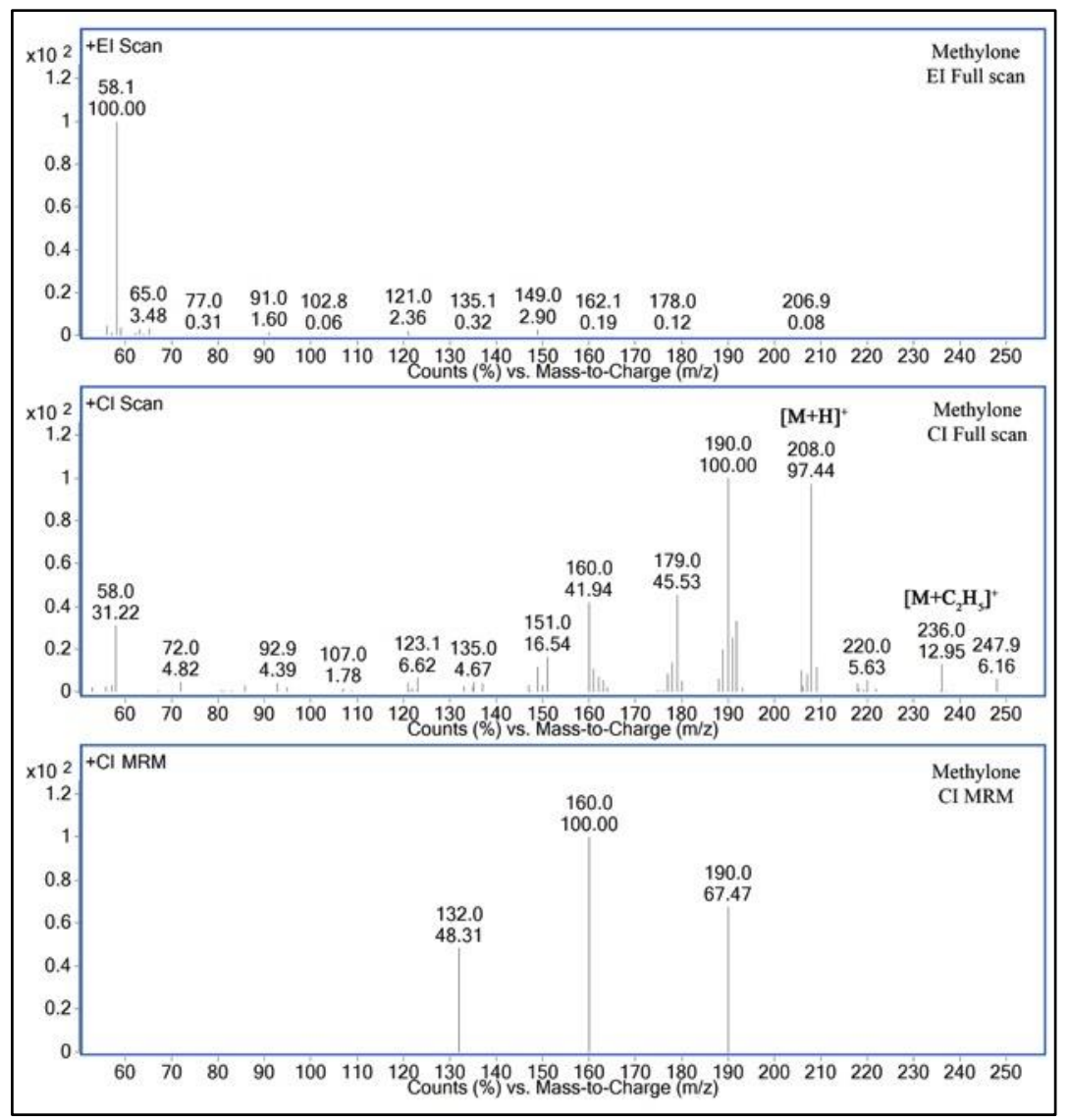

Figure 40. Mass spectra of methylone by EI full scan (top), CI full scan (middle), and CI MRM (bottom). 


\subsubsection{Conclusions}

The qualitative analysis of 244 NPS was performed by GC-MS/MS with two different ionization sources, EI and CI. Of 244 substances, only 34 NPS are selectively shown for the results from the evaluation of GC-MS/MS as an alternative confirmatory technique. The conventional method for the analysis of NPS using GC-MS with an EI source suffers from the lack of spectral information because of the structural similarity between related NPS. In order to solve the problem, methods for the analysis of these substances with enhanced specificity are necessary to facilitate the identification of these compounds. It is evident that the implementation of the CI source is beneficial for the easier identification of these emerging NPS that are extensively fragmented in the EI source with the level of below parts per million detection capabilities. The analysis of seized drug samples has shown that the use of the CI source makes relatively easier than the EI source for the identification of the unknown sample. In addition, the transitions of a specific precursor ion monitored by MRM mode provided additional spectral information to identify the isomers of interest. Consequently, it is reported that a sensitive and selective method using GC-MS/MS can be used for the positive identification of these increasingly important NPS at the level of qualitative seized-drug analysis. The corresponding mass spectra from these substances are reported for the potential use of supplemental spectral library database. The qualitative analysis of 34 compounds by GC-MS/MS has been published in peer-reviewed literature [133]. 


\subsection{Gas Chromatography Quadrupole Time-of-Flight}

As discussed previously in 2.3.3.2, a high-resolution quadrupole time-of-flight (QTOF) mass analyzer has been commonly coupled to the LC system in a variety of applications. In this section 5.2, a QTOF mass analyzer hyphenated with the GC system (Figure 41) was utilized for the qualitative analysis of emerging NPS. The relatively new analytical instrument, especially Agilent GC-QTOF-MS, provides high resolution full scan and targeted MS/MS spectra with accurate mass, improved mass resolution of TOF allowing the extracted ion chromatograms with narrower mass window, and a scan rate as fast as $50 \mathrm{~Hz}$ [136]. With these advantages mentioned, GC-QTOF-MS instrument has been mostly applied in food safety for screening of pesticide residues [136-137] as well as in environmental science for screening and identification of organic pollutants in water [138-139].

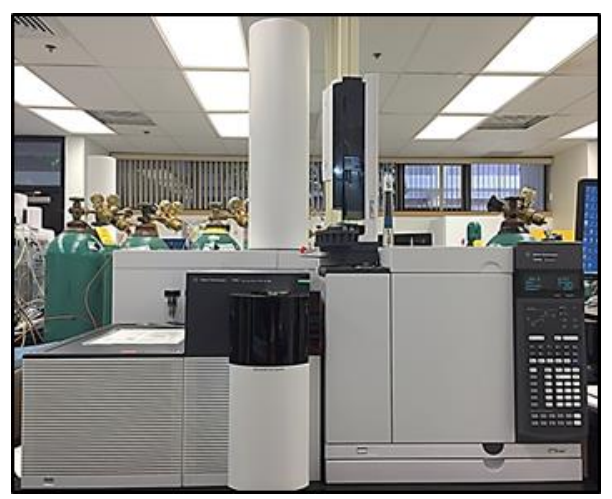

Figure 41. Picture of GC-QTOF-MS system.

The QTOF mass analyzer for the GC system was developed with the idea of combining the advantages of both a triple quadrupole mass analyzer for the GC system and a QTOF mass analyzer for the LC system [140]. Figure 42 shows the schematic of the QTOF mass analyzer, which is partially different from the QTOF-MS designed for 
the LC system described in Figure 17. The main difference between two QTOF mass analyzers is the position of ionization source. In GC-QTOF-MS, the ionization sources, EI and CI, are under high vacuum, while the ESI or APCI sources used in LC-QTOF-MS are under atmospheric pressure. The GC-QTOF-MS system is equipped with a removable ion source, which enables changing of the ionization source without venting the entire mass analyzer (Figure 43). Although the automated removable ion source procedure is composed of multiple steps of purging, venting, pumping down and gate opening/closing, it can be done within 30 minutes.

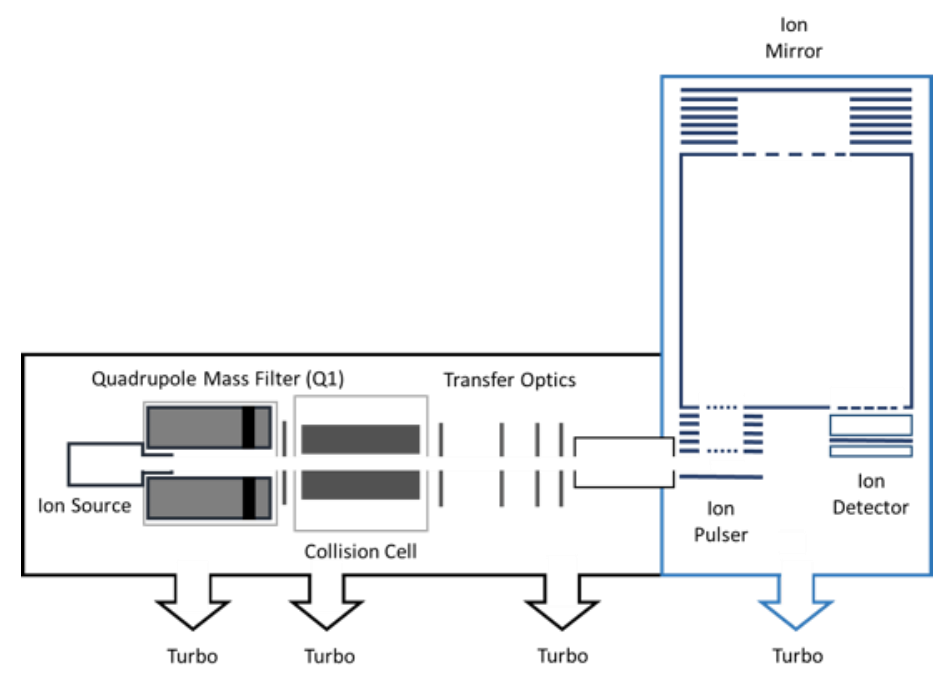

Figure 42. Schematic of a high resolution QTOF mass analyzer for GC-MS. Adapted and reproduced from [140].

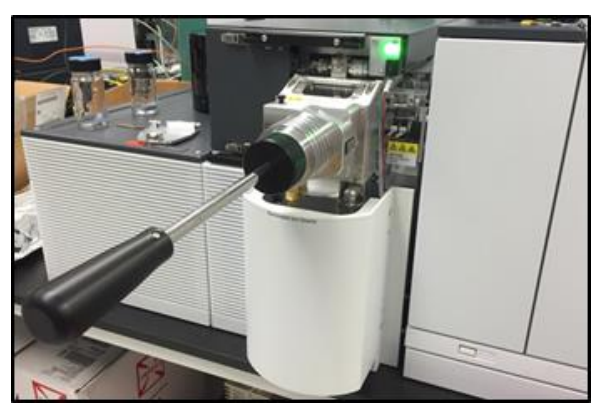

Figure 43. Picture of removable ion source apparatus. 
Recently, the analysis of NPS has encountered difficulties because of the increased number of newly identified substances, the absence of reference standards, and the complexity of analytes in mixtures or biological matrices [141]. It is believed that chromatography coupled with high resolution mass spectrometry plays an important role in identifying analytes in complex matrices [141]. Therefore, the qualitative analysis of NPS using the GC-QTOF-MS instrument was demonstrated in this dissertation for the first time.

\subsubsection{Qualitative Analysis of NPS standards}

A total number of 256 NPS were analyzed by GC-QTOF-MS in both EI and CI mode to create a database. Among total compounds, the same set of 244 NPS was also analyzed in the previous section and the list of these substances can be found in Appendix 1. The additional 12 substances were also provided by Cayman Chemical and were prepared with different organic solvent at different target concentrations as listed in Table 10. These additional compounds were further diluted with methanol at $10 \mu \mathrm{g} / \mathrm{mL}$, which were used as a working solution. The operating conditions for the GC system was already developed and optimized during the previous study by GC-MS/MS, but only a couple of parameters, such as injection volume, injection mode, and temperature of transfer line, were modified as described in 3.4.

The analysis of 256 NPS was performed after compounds were divided into two groups, 157 synthetic cannabinoids and 99 additional types of NPS. On the basis of the previous study by GC-MS/MS, it was revealed that most synthetic cannabinoids could be 
readily identified with the presence of molecular information in EI mass spectra, while most synthetic cathinones are ambiguously identified because of extensive fragmentation in the EI source. Therefore, 157 synthetic cannabinoids were first analyzed by GCQTOF-MS with the EI source. As a result, it was found that $78 \%$ of synthetic cannabinoids showed molecular information in EI mass spectra with high mass accuracy less than $4.8 \mathrm{ppm}(1.3 \mathrm{mDa})$ as shown in Figure 44 (a). The molecular ion was not found or shown in very low relative abundance for the other $20 \%$ of these compounds, which may result in an ambiguous identification by GC-QTOF-MS in EI mode. The other 2\% of synthetic cannabinoids showed either poor mass accuracy for the molecular ion or very low abundance in the chromatogram.

Table 10. List of additional 12 substances analyzed by GC-QTOF-MS.

\begin{tabular}{ccccc}
\hline & NPS & \multicolumn{1}{c}{$\begin{array}{c}\text { Molecular } \\
\text { Formula }\end{array}$} & $\begin{array}{c}\text { Concentration } \\
(\mu \mathrm{g} / \mathrm{mL})\end{array}$ & Solvent \\
\hline \hline 1 & $\mathrm{AM} 1241$ & $\mathrm{C}_{22} \mathrm{H}_{22} \mathrm{IN}_{2} \mathrm{O}$ & 1000 & $\mathrm{MeOH}$ \\
2 & $\mathrm{AM630}$ & $\mathrm{C}_{23} \mathrm{H}_{25} \mathrm{IN}_{2} \mathrm{O}_{3}$ & 1000 & $\mathrm{DMSO}$ \\
3 & $\mathrm{CB}-25$ & $\mathrm{C}_{23} \mathrm{H}_{21} \mathrm{NO}$ & 1000 & $\mathrm{MeOH}$ \\
4 & $\mathrm{CB}-52$ & $\mathrm{C}_{25} \mathrm{H}_{41} \mathrm{NO}_{3}$ & 1000 & $\mathrm{MeOH}$ \\
5 & $\mathrm{CB}-86$ & $\mathrm{C}_{26} \mathrm{H}_{43} \mathrm{NO}_{3}$ & 10000 & $\mathrm{EtOH}$ \\
6 & $\mathrm{CP} 47,497-$ para-quinone analog & $\mathrm{C}_{21} \mathrm{H}_{32} \mathrm{O}_{3}$ & 1000 & $\mathrm{MeOH}$ \\
7 & JP104 & $\mathrm{C}_{25} \mathrm{H}_{30} \mathrm{~N}_{2} \mathrm{O}_{3}$ & 1000 & $\mathrm{MeOH}$ \\
8 & MDA 77 & $\mathrm{C}_{21} \mathrm{H}_{23} \mathrm{~N}_{3} \mathrm{O}_{3}$ & 1000 & $\mathrm{MeOH}$ \\
9 & URB447 & $\mathrm{C}_{25} \mathrm{H}_{21} \mathrm{ClN}_{2} \mathrm{O}$ & 1000 & $\mathrm{MeOH}$ \\
10 & 4-Fluoroisocathinone & $\mathrm{C}_{9} \mathrm{H}_{10} \mathrm{FNO}_{2}$ & 1000 & $\mathrm{MeOH}$ \\
11 & Ketazolam & $\mathrm{C}_{20} \mathrm{H}_{17} \mathrm{ClN}_{2} \mathrm{O}_{3}$ & 1000 & $\mathrm{DMSO}$ \\
12 & Methylhexanamine & $\mathrm{C}_{7} \mathrm{H}_{17} \mathrm{~N}^{2}$ & 1000 & $\mathrm{MeOH}$ \\
\hline
\end{tabular}




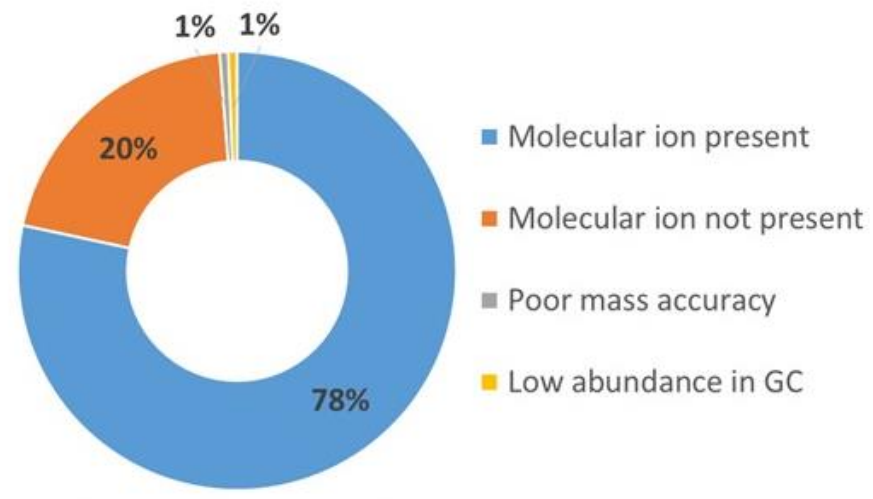

(a) Synthetic cannabinoids

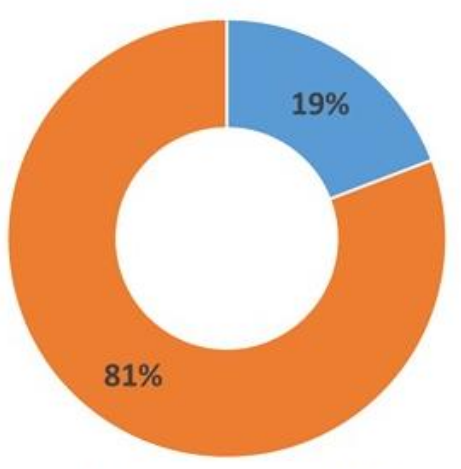

(b) Additional types of NPS

Figure 44. Summary of results from (a) synthetic cannabinoids and (b) additional types of NPS analyzed by GC-QTOF-MS in EI full scan.

The other set of 99 NPS was also analyzed by GC-QTOF-MS with the EI source. Figure 44 (b) shows that molecular ions were present only for $19 \%$ of the subset of additional types of NPS with mass accuracy less than $4.78 \mathrm{ppm}(1.3 \mathrm{mDa})$. These substances were mainly piperazines or phenethylamines ( $2 \mathrm{C}$ class). However, molecular ions were not observed in the majority of NPS (81\%) in the subset. Those compounds shown extensive fragmentation in EI mass spectra were analyzed in CI full scan mode with the expected presence of protonated molecular ions. In the subset of 99 NPS, molecular ions were successfully identified for most of them, except 2-fluoromethcathinone and 4fluoroisocathinone. Mass accuracy of these 97 NPS was between 0.32 and $10.24 \mathrm{ppm}$, which corresponds to 0.0 - $2 \mathrm{mDa}$. Although the degree of mass error in CI mode was greater than EI mode, the utilization of the CI source was still beneficial to identify those substances that were extensively fragmented with the EI source. In addition, the acquisition of molecular information with accurate mass is essential in terms of unknown analysis. 


\subsubsection{Determination of Detection Limits}

The limits of detection (LODs) were determined to provide a figure of merit as an analytical tool in the analysis of NPS using GC-QTOF-MS in EI full scan mode. Calibration solutions containing $\mathrm{CP}-47,497$ and $\mathrm{JWH}-250$ were prepared from 100 to $3200 \mathrm{ng} / \mathrm{mL}$ with methanol. Each solution was injected three times and average peak area from each injection was calculated after quantifier ions were extracted. Since the QTOF-MS gives accurate mass, a range of target exact mass was extracted for quantifier and qualifier ions as shown in Table 11. The minimum criteria for both quantifier and qualifier ions was a threefold signal-to-noise ratio $(\mathrm{S} / \mathrm{N})$.

Table 11. Ranges of quantifier and qualifier ions for CP-47,497 and JWH-250 during the determination of detection limits.

\begin{tabular}{cccc}
\hline Analyte & Quantifier Ion & Qualifier Ion 1 & Qualifier Ion 2 \\
\hline \hline CP-47,497 & $215.0000-215.2000$ & $318.1000-318.4000$ & $233.1000-233.3000$ \\
JWH-250 & $214.1000-214.3000$ & $144.0000-144.3000$ & $116.0000-116.3000$ \\
\hline
\end{tabular}

Calibration curves were plotted in the linear range of 800 to $3200 \mathrm{ng} / \mathrm{mL}$ for $\mathrm{CP}-47,497$ and 400 to $3200 \mathrm{ng} / \mathrm{mL}$ for JWH-200 as shown in Figure 45. The LOD calculation was made on the basis of three times of the standard error divided by the slope of linear correlation for each compound. The LODs were determined to be $370.0 \mathrm{ng} / \mathrm{mL}$ and $145.3 \mathrm{ng} / \mathrm{mL}$ for $\mathrm{CP}-47,497$ and $\mathrm{JWH}-250$, respectively, on average from the three different days. The determined detection capabilities of GC-QTOF-MS were higher than that of GC-MS/MS from the previous section for those two compounds, CP-47, 497 and 
JWH-250. The difference is mainly because of the scan modes used during the evaluation, full scan (GC-QTOF-MS) and MRM (GC-MS/MS) modes.

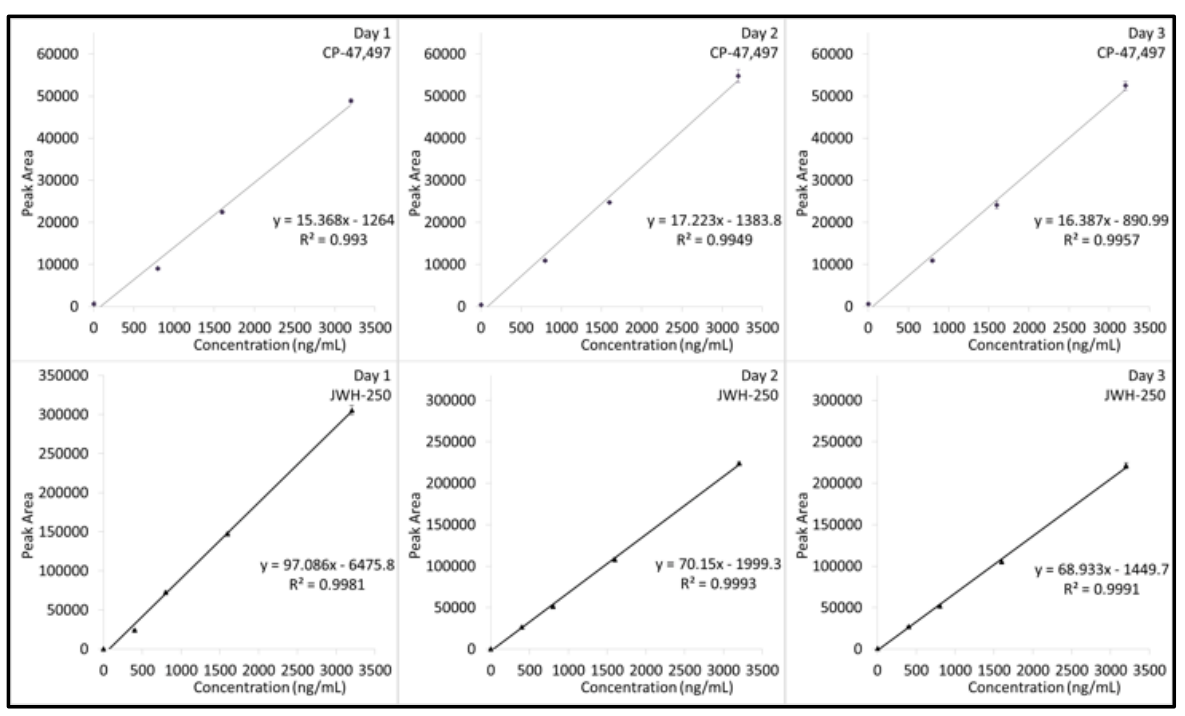

Figure 45. Calibration curves for CP-47,497 and JWH-250 by GCQTOF-MS with the EI source in three different days.

\subsubsection{Analysis of Seized Samples}

To evaluate the applicability of GC-QTOF-MS in the qualitative analysis of NPS, two seized drug samples analyzed by the Barringer IMS and DART-QTOF-MS were selected and investigated in this section. The analysis of these two samples was performed using GC-QTOF-MS in EI full scan mode in accordance with the method used for the analysis of NPS standards. Figure 46 shows the chromatograms and mass spectra of two seized samples. In EI scan mass spectra, unambiguous identification was not possible because of lack of molecular information. It was also found that the identification of unknown is even more challenging when it comes to a mixture. As addressed in the previous section in 5.1, the implementation of the CI source in GC- 
QTOF-MS will facilitate the unambiguous identification with the presence of protonated molecular ions. However, the separation of multi-compounds mixture can be still challenging in mass spectra when one compound is significantly prevalent over the other compound unless a mixture can be separated in chromatograms. In terms of rapid separation capability, the Barringer IMS is more efficient to characterize mixtures with multiple compounds for the rapid screening capability and ease of instrument operation.

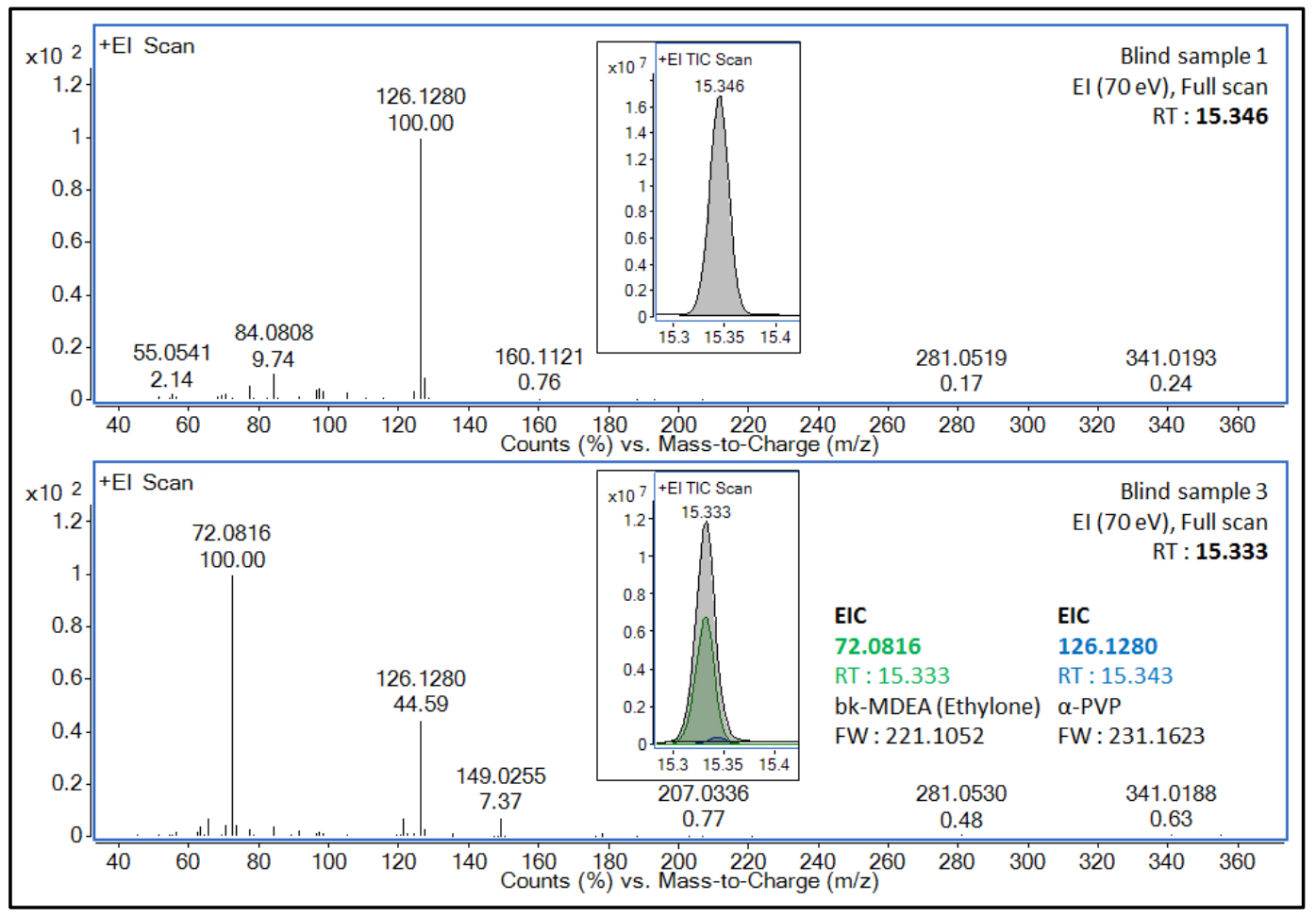

Figure 46. Chromatograms and mass spectra of two seized drug samples by GC-QTOFMS in EI full scan mode.

\subsubsection{Conclusions}

Continuing from the analysis by GC-MS/MS, a GC system coupled to a state-ofthe-art high resolution QTOF mass spectrometer was utilized in the analysis of emerging NPS in both EI and CI mode for the first time. Evidently, the use of CI in GC-MS is 
advantageous for the unambiguous identification of compounds that are easily fragmented and lacking molecular information in EI mass spectra. Although the analysis of mixture is challenging when components are not separated in chromatograms, the presence of protonated molecular ions with accurate mass can facilitate the identification of multiple components in CI mode. There are a number of challenges associated with the analysis of these NPS, including the endless emergence of newly identified substances, presence of various isomers, complexity of analytes and matrices, and lack of reference standards for new substances. Therefore, the application of high resolution mass spectrometry in drug analysis, especially for emerging NPS, will be beneficial with high sensitivity and selective capabilities of the GC-QTOF-MS instrument.

\subsection{Electrospray Ionization Ion Mobility Spectrometry Mass Spectrometry}

The coupling of IMS with MS provides a number of advantages over traditional IMS with a radioactive ionization source as briefly described in previous section 2.3.2.2. Consequently, the development of this hyphenated technique has enabled many new applications of IMS with the identification capabilities of unknowns on the basis of the mobility and mass-to-charge ratio of an ion [70]. These applications include the analysis of biomolecules [76, 79-80], the detection and identification of drugs [32-35, 142], and the analysis of explosives [35, 143]. Many fundamental studies have been done utilizing ESI-IMS-MS for charge competition during the ionization process [34], the selection of drift gas for selectivity [144], the separation mechanism of chiral compounds [142], and the speciation effects of solvent system [35]. Recently, a couple of studies on new 
synthetic drugs referred to NPS have been reported using the IMS coupled to different types of MS [116-118]. In this section, a number of synthetic cathinones were investigated using a commercially available high performance IMS (Excellims, Acton, MA, USA) coupled with QC-150 quadrupole (Extrel CMS, Pittsburgh, PA, USA), which offers a resolving power of 60 to 100 (Figure 47) [145].

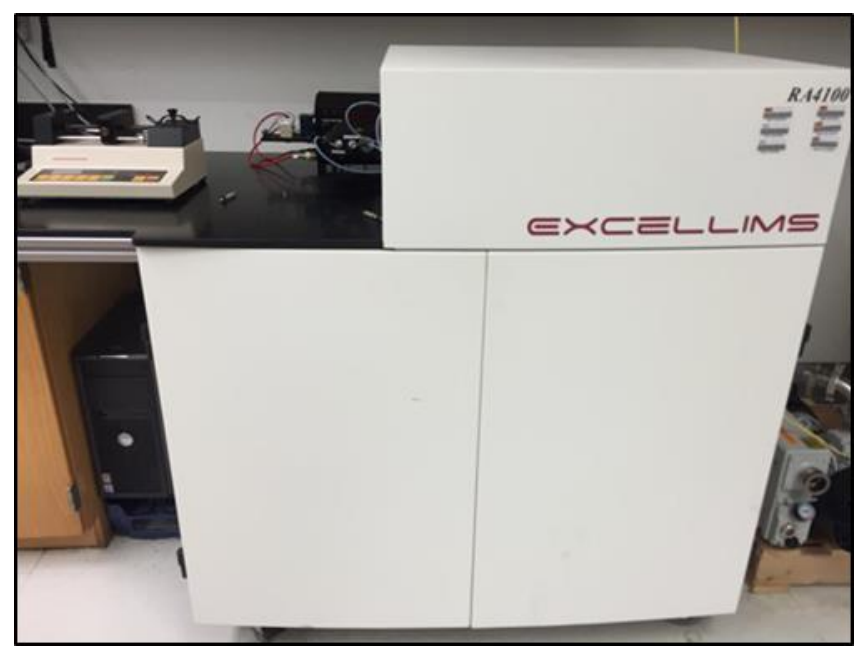

Figure 47. Excellims ESI-IMS-MS (RA4100).

In this ESI-IMS-MS system, an air-cooled ESI source is utilized as shown in Figure 48. The source is different than a water-cooled ESI source that was originally developed and used in the study by Wu et al. [76]. Nevertheless, the purpose of the air-cooled ESI source is to cool the spray needle from the heated drift gas in order to prevent the evaporation of solvent inside the needle. The electrospray released from the tip of spray needle is moved toward a mesh screen by the electrical potential applied between the needle and the mesh screen. Finally, the charged droplets are introduced into the desolvation region where solvent is evaporated during the migration of droplets through 
an electric field [76]. A close-up picture of the air-cooled ESI source coupled to the IMS is also shown in Figure 49.

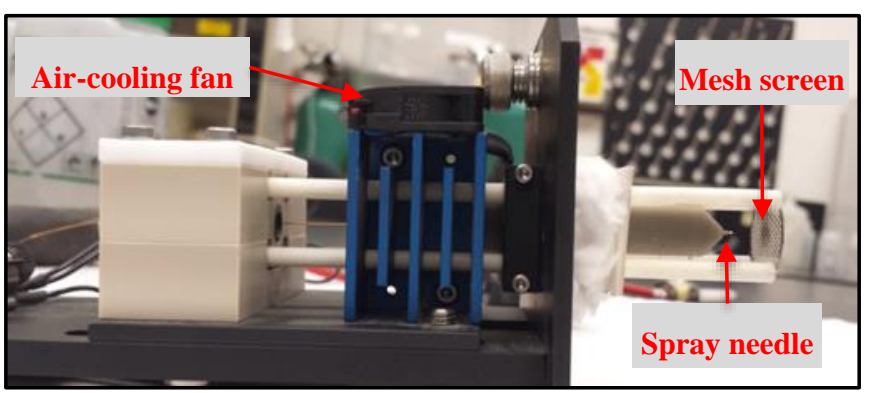

Figure 48. An air-cooled ESI source used in the Excellims ESI-IMS-MS.

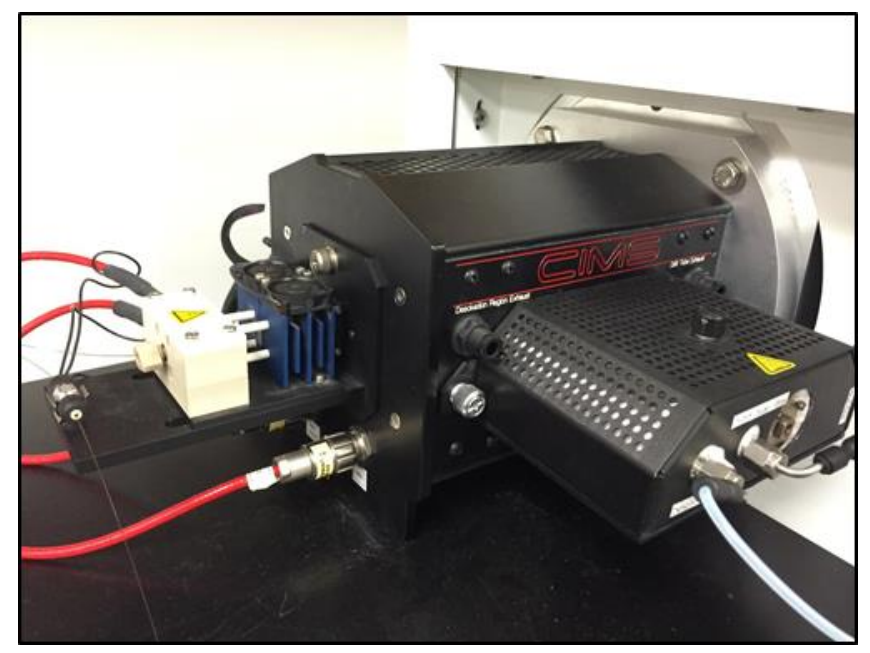

Figure 49. Close-up of a cooled ESI source coupled to the IMS.

\subsubsection{Optimization of Solvent System}

In the ESI source, only a small portion of analytes are ionized generating gas-phase ions that are transported into the mass analyzer [146]. Although ESI is not the best ionization technique to produce gas-phase ions, the capability of soft ionization and less restrictions on the masses of anayltes have caused the ESI source to become one of the 
most commonly used ionization techniques in modern mass spectrometers. Ionization efficiency of ESI describes how competently the analytes or ions can produce gas-phase ions during the ESI process [146]. A number of studies have actively evaluated the ionization efficiency in ESI with the application of IMS [32, 34-35]. It is demonstrated that the addition of an acetic acid modifier in the gas phase improved the intensity of detected ions [32, 34]. Another study investigated the effect of the solvent system comprehensively when the ESI source is implemented with IMS-MS [35]. A wide range of different solvent compositions was evaluated with different amounts of acidic modifier. In addition, the different types of ionic species in both positive and negative modes were determined thoroughly. In this section, different solvent compositions for synthetic cathinones were evaluated for the greatest ionization efficiency in the ESI source. Three synthetic cathinones, 4-MEC, methedrone, and MDPV, were selected for the solvent study on the basis of the functional group, a methyl group on the amine, ethyl group on the amine, and pyrrolidine group (Figure 3). The previous study performed by Howard et al. showed that the optimal solvent system was methanol and water in a ratio of 80 to $20(v / v)$ with $2.5 \%$ of formic acid [35]. Also, it is recommended to contain less water in the solvent system. Therefore, only a small range of methanol and water ratio, 80:20 $(v / v)$ and 90:10 $(v / v)$, was evaluated with the addition of different amounts of formic acid (FA) ranging from 0 to $2.5 \%$. Each solvent was used to prepare a sample solution containing a target compound. The measurement of the sample solution was performed in triplicate to obtain average intensity. As shown in Figure 50, addition of an acidic modifier improved the average intensity of analytes compared to other solutions with the same solvent composition. Although each compound showed different 
ionization efficiency, the solvent composition of 80:20 (v/v) with 2.5\% FA was selected because of the consistent intensity throughout all three compounds.

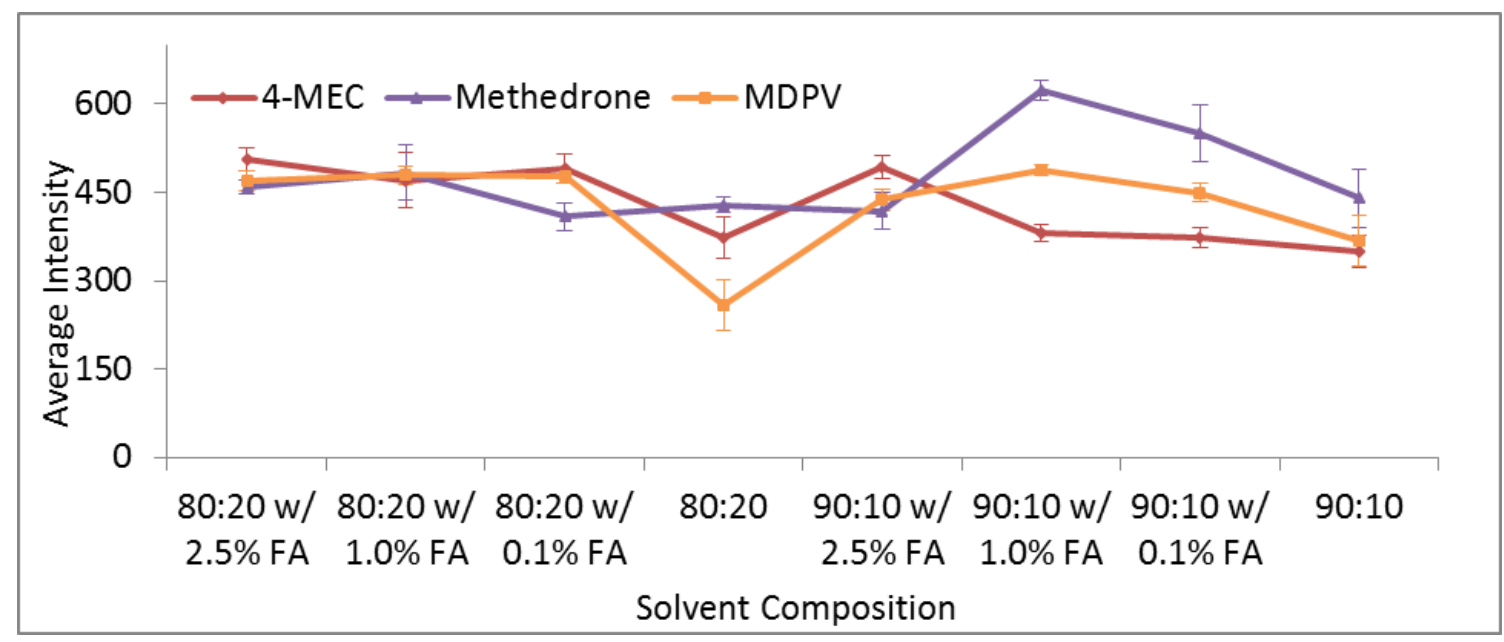

Figure 50. Optimization of elctrospray ionization solvent system for three different groups of synthetic cathinones. The consistent average intensity was observed the solvent composition of $80: 20$ with $2.5 \%$ FA for three compounds.

\subsubsection{Analysis of synthetic cathinones}

The identification capability of 6 representative synthetic cathinones, including 4MMC, 4-MEC, 3-FMC, methedrone, methylone, and MDPV, was investigated using the Exellims ESI-IMS-MS system. Table 12 shows the analytes of interest with the drift time and reduced mobility $\left(K_{0}\right)$ resulting from the evaluation along with the known $K_{0}$ values from literatures. The drift time of each analyte was measured in triplicate before and after running a blank solvent. The $K_{o}$ of these analytes $\left(t_{d u k n}\right)$ was calculated on the basis of Equation 4 as described in 2.3.2. Before the analysis of these compounds, cocaine was analyzed under the same conditions as a chemical standard of each synthetic cathinone. The obtained drift time of cocaine was used as the drift time of the reference 
standard, $t_{d}$ ref, and the $K_{0}$ of cocaine $\left(K_{0} \mathrm{ref}, 1.19 \mathrm{~cm}^{2} / \mathrm{Vsec}\right)$ was from the literature.[147] The calculated $K_{0}$ of 6 synthetic cathinones by ESI-IMS-MS were compared to the values obtained by Barringer IMS (4.1.1) as well as previously reported in the literature. It is shown that the $K_{0}$ of MDPV is consistent with the results from the Barringer IMS and the literature. For 4-MMC, 4-MEC, and methylone, the obtained $K_{0}$ was slightly different from the Barringer IMS results and the literature $\left( \pm 0.05 \mathrm{~cm}^{2} / \mathrm{Vsec}\right)$. The drift time shift between analyses may have affected variations in the $K_{0}$ values of 4-MMC, 4-MEC, and methylone. However, the calculated $K_{0}$ of 3-FMC showed an exceptional difference with the previous result by the Barringer IMS. It is assumed that the main component of the ion swarm produced from the ${ }^{63} \mathrm{Ni}$ radioactive source is different from the ESI source.

Table 12. List of 6 NPS analyzed by ESI-IMS-MS and the results from the study.

\begin{tabular}{ccccc}
\hline NPS & $\begin{array}{c}\text { Molecular } \\
\text { Weight }(\mathrm{amu})\end{array}$ & $\begin{array}{c}\text { Drift Time } \\
(\mathrm{ms})\end{array}$ & $\begin{array}{c}K_{0} \\
\left(\mathrm{~cm}^{2} / \mathrm{Vsec}\right)\end{array}$ & Reference $K_{0}\left(\mathrm{~cm}^{2} / \mathrm{Vsec}\right)$ \\
\hline \hline 4-MMC & 177.24 & 9.68 & 1.45 & $1.499[116]$ \\
4-MEC & 191.70 & 10.01 & 1.41 & $1.447[115], 1.30^{*}[117], 1.41[118]$ \\
3-FMC & 181.21 & 9.75 & 1.44 & $1.530[115]$ \\
Methedrone & 193.24 & 9.93 & 1.42 & \\
Methylone & 207.23 & 10.13 & 1.39 & $1.435[115], 1.435[116], 1.28^{\dagger}[117]$ \\
MDPV & 275.34 & 11.65 & 1.21 & $1.199[115], 1.201[116], 1.18[117]$ \\
\hline
\end{tabular}

*: 1.30 for $m / z 192.14$ in APCI and ESI and 1.41 for $m / z 190.12$ in APCI

$\dagger: 1.28$ for $m / z 208.10$ in APCI and ESI and 1.35 for $m / z 206.08$ in APCI

Ion mobility spectra of six synthetic cathinones are shown in Figure 51. Each compound was individually analyzed via the direct infusion using a gas-tight syringe delivering $100 \mu \mathrm{L}$ at a flow rate of $3 \mu \mathrm{L} / \mathrm{min}$ as previously described in section 3.5. 
Although the amount of sample loaded into the syringe was $100 \mu \mathrm{L}$, the sample used in the analysis is much less than that. The less amount of loaded sampled is because a sample was pumped into the system before the acquisition of the ion mobility spectrum and full scan mass spectrum in order for pre-conditioning of the injection capillary column and the ESI source. The background ion peak (BIP) or solvent peak is commonly observed in ion mobility spectra acquired by ESI-IMS-MS. The BIP is a result of the formation of methanol clusters in positive mode, corresponding to the most dominant ions of $m / z 65\left[\left(\mathrm{CH}_{3} \mathrm{OH}\right)_{2} \mathrm{H}^{+}\right]$and $97\left[\left(\mathrm{CH}_{3} \mathrm{OH}\right)_{3} \mathrm{H}^{+}\right][35]$.

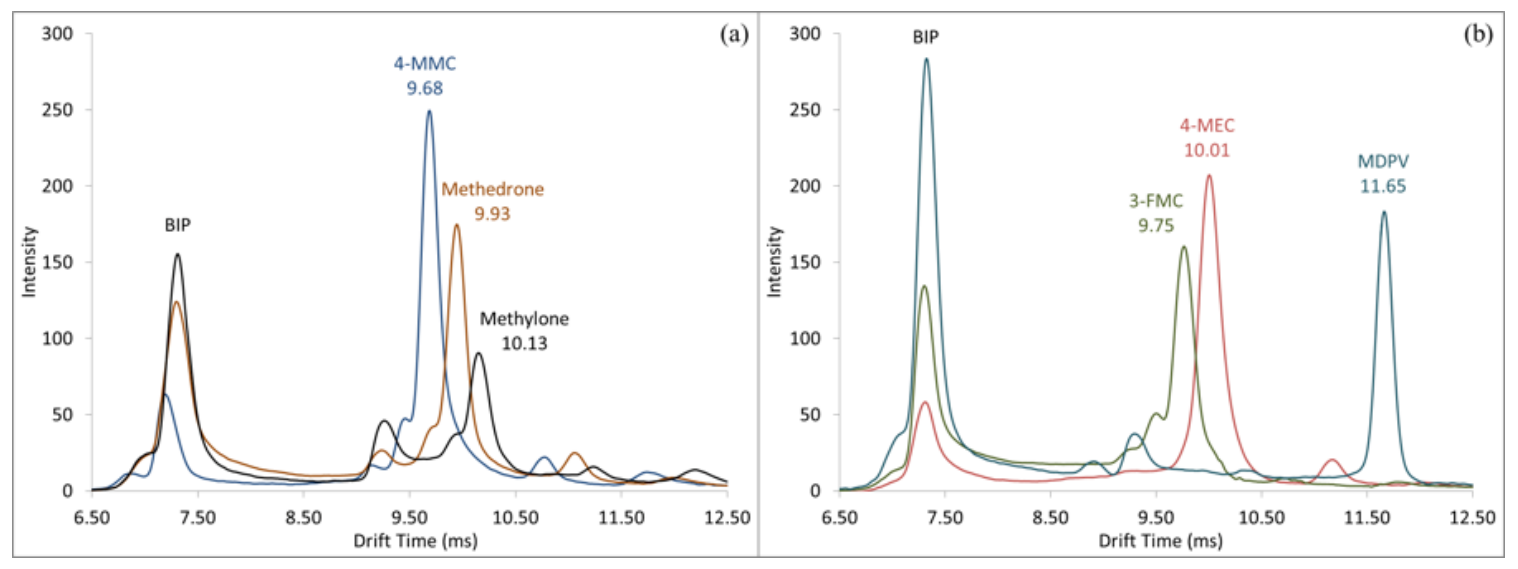

Figure 51. Overlaid ion mobility spectra of 6 synthetic cathinones by direct infusion. Each substance was successfully detected by ESI-IMS-MS.

Figure 52 shows full scan mass spectra of 6 synthetic cathinones analyzed in the investigation. Each analyte was successfully identified with the presence of the protonated molecular ion as a base peak in each mass spectrum. In addition, the selected ion mode, which monitors the protonated molecular ion, was also simultaneously performed during the acquisition of both ion mobility spectra and scan mass spectra. Although it was not presented in this section, the plot of selected ion monitoring also 
showed a good agreement with the ion mobility spectrum. Therefore, the results suggest that ESI-IMS-MS can be potentially used as a confirmation method in the rapid analysis of synthetic cathinones with a total analysis time less than five minutes.

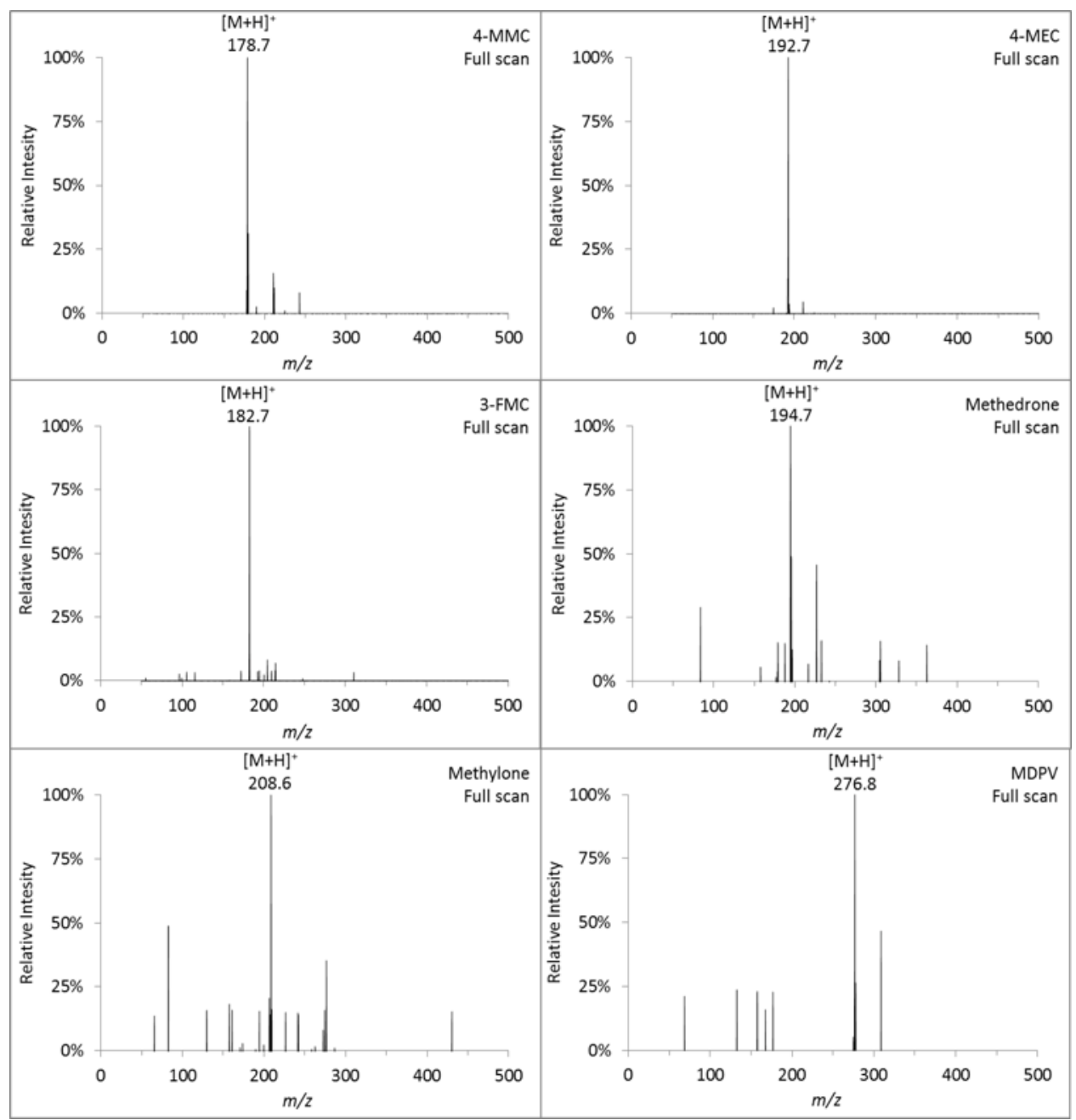

Figure 52. Full scan mass spectra of 6 synthetic cathinones by ESI-IMS-MS. Each substance was identified with the presence of a protonated molecular ion. 


\subsubsection{Separation of two-analyte mixtures}

The separation capability of ESI-IMS-MS was subsequently evaluated by analyzing mixtures of two compounds. A mixture of $4-\mathrm{MMC}$ and methedrone at $100 \mu \mathrm{g} / \mathrm{mL}$ was first analyzed by ESI-IMS-MS and its ion mobility spectrum and full scan mass spectrum are shown in Figure 53. Although the protonated molecular ions of both compounds were observed in the mass spectrum, the separation of two analytes was not accomplished in the ion mobility spectrum. It might be because the drift times of 4-MMC (9.68 ms) and methedrone $(9.93 \mathrm{~ms})$ were too close each other. In addition, it is important to note that the BIP (solvent peak) was not observed in the ion mobility spectra, which indicates the amount of charge available was completely used to ionize the analytes at high concentrations [34].

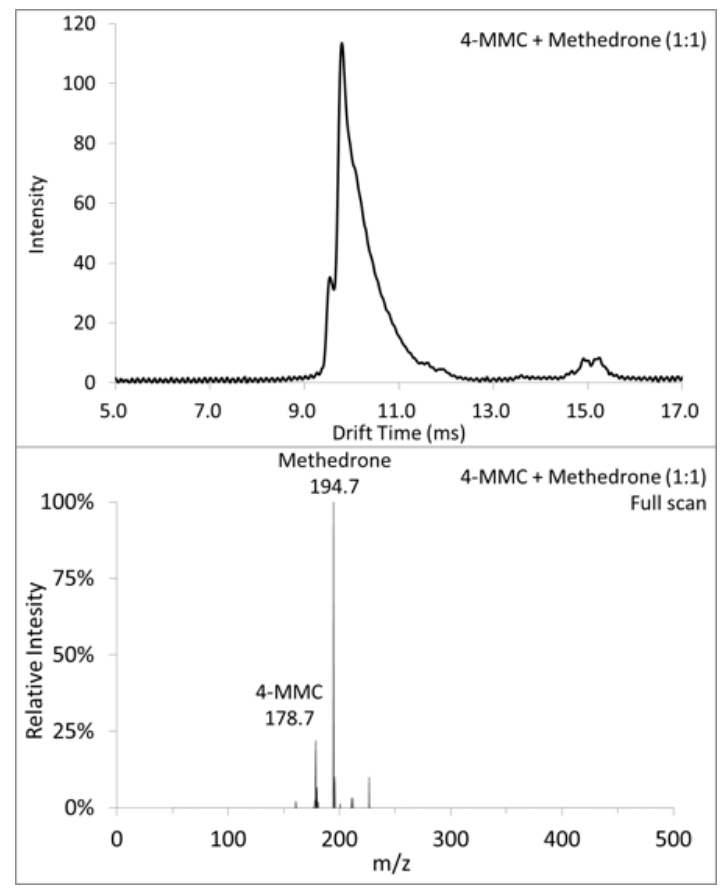

Figure 53. Ion mobility spectrum (top) and mass spectrum (bottom) of a mixture of 4-MMC and methedrone by ESI-IMS-MS. The presence of two compounds in the mixture was confirmed with protonated molecular ions. 
In order to investigate the separation capabilities of compounds that have essentially distinguished drift times, methylone (10.13 ms) and MDPV (11.65 ms), were selected for the analysis of mixtures. Figure 54 shows overlaid ion mobility spectra of two mixtures with mass spectra from each mixture. These two mixtures are composed of the same compounds, but different relative concentrations of MDPV. First, the mixture of methylone and MDPV at $100 \mu \mathrm{g} / \mathrm{mL}$ was prepared and analyzed by ESI-IMS-MS. Unexpectedly, it was shown that only one peak potentially associated with MDPV was observed both in the ion mobility spectrum and mass spectrum, which indicates that there is charge competition between two analytes preferentially ionizing MDPV in the solution. The charge competition has been commonly found in the ESI process over many years as a main limitation in the analysis of mixtures in ESI-IMS-MS [34-35]. Since the ionization occurs in a solution before a sample is introduced into the IMS through the ESI source, it may be inevitable to avoid charge competition unless a capillary column is added prior to ionization. At least to minimize the charge competition between methylone and MDPV, another mixture was prepared with different concentrations of MDPV (i.e. methylone at $100 \mu \mathrm{g} / \mathrm{mL}$ and MDPV at $20 \mu \mathrm{g} / \mathrm{mL}$ ), which is shown as 'bold line' in Figure 54. As a result, two compounds were successfully separated in the ion mobility spectrum and the protonated molecular ions from both analytes were observed in the full scan mass spectrum. 


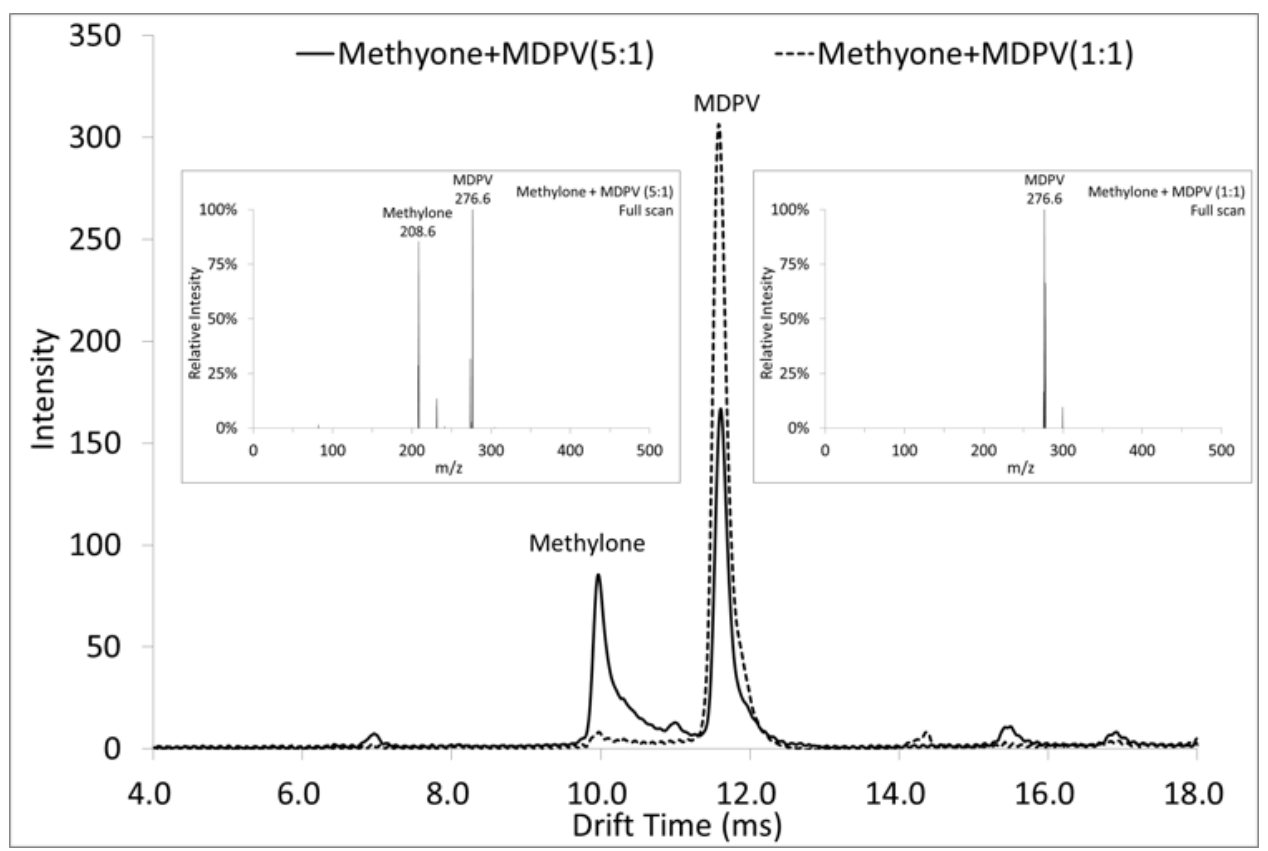

Figure 54. Ion mobility spectra and full scan mass spectra of two mixtures with different relative concentration. Charge competition between methylone and MDPV were observed in mixture analysis.

The analysis of mixtures were also performed for other compounds as shown in Figure 55. A total number of four mixtures were prepared by combining MDPV with four other synthetic cathinones at the different concentration of each analyte, $20 \mu \mathrm{g} / \mathrm{mL}$ for MDPV and $100 \mu \mathrm{g} / \mathrm{mL}$ for other compounds. It is shown that the separation of these 4 mixtures were successfully achieved under the previously described conditions. Other two-analyte mixtures were prepared using five synthetic cathinones, excluding MDPV, and analyzed to investigate separation capabilities. Unfortunately, the baseline separation of mixtures was not successful because of the drift time similarity between these compounds. Despite the limited cases, the separation of two-analytes mixtures was possible with mass spectral information using ESI-IMS-MS. 


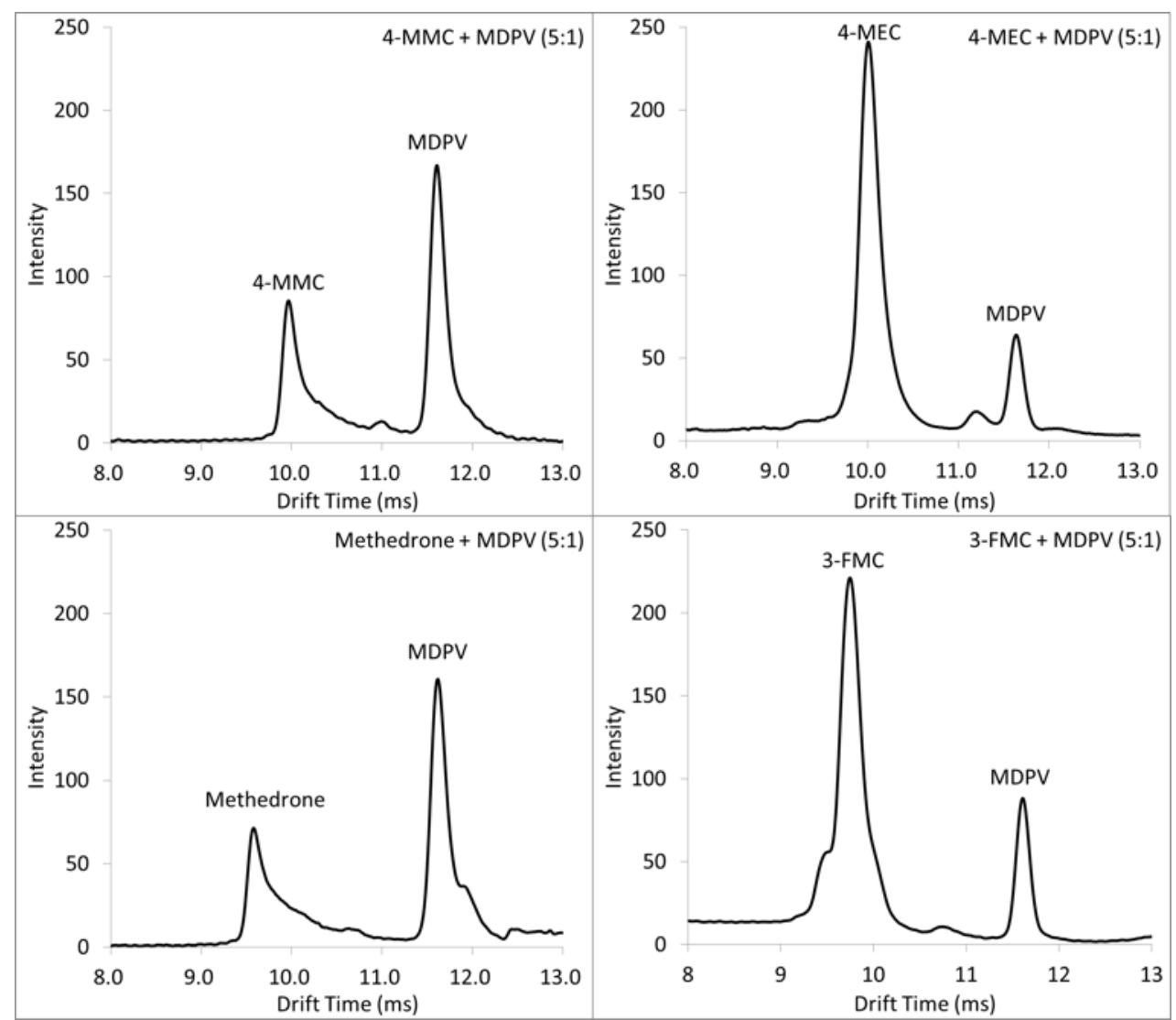

Figure 55. Ion mobility spectra of mixtures with a different ratio of two compounds in concentration.

\subsubsection{Determination of Detection Limits}

The limits of detection (LODs) for these six synthetic cathinones were determined to address figures of merit for the application of ESI-IMS-MS. The calibration solutions were prepared in the range of 5 to $100 \mu \mathrm{g} / \mathrm{mL}$ and analyzed in three replicates by Excellims ESI-IMS-MS. The ratio of max intensity of analyte to that of solvent peak (BIP) was used for the instrument response because the absolute response varied between runs even from the same solution. The calibration curves for 6 compounds were constructed according to the ratio of analyte peak to solvent peak versus the 
concentration of each sample as shown in Figure 56. In the different linear ranges between these analytes, each compound showed a good linearity of $\mathrm{R}^{2}>0.98$. The LODs was calculated as three times the standard deviation of the intercept divided by the slope of the linear regression as described previously. It was determined that the LODs were 19.1, 10.6, 10.7, 6.75, 13.5, and $2.0 \mu \mathrm{g} / \mathrm{mL}$ for 4-MMC, 4-MEC, 3-FMC, methedrone, methylone, and MDPV, respectively. The comparison of detection limits between analytical techniques from the previous chapters shows that ESI-IMS-MS is less sensitive than other techniques.

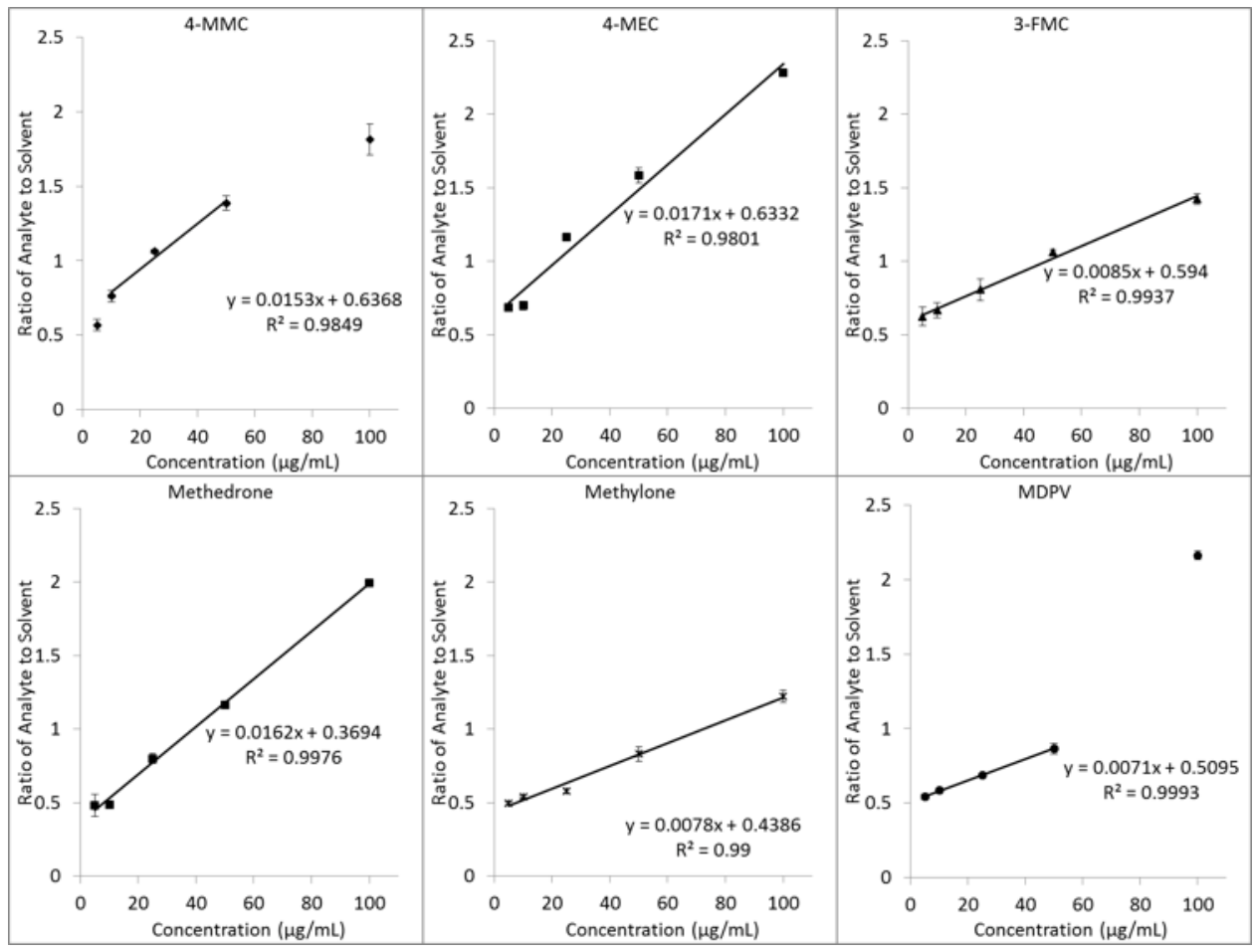

Figure 56. Calibration curves of six synthetic cathinones by ESI-IMS-MS. A good linearity was shown for each substance in different linear ranges. 


\subsubsection{Analysis of Seized Samples}

A total number of four seized drug samples provided by a local forensic laboratory were also analyzed by ESI-IMS-MS. These four samples were prepared in methanol and the same approach was applied in the analysis of seized samples as described in the previous evaluation for the analysis of standard solutions. Figure 57 shows the results from the blind study for one of the seized samples. The seized sample was successfully detected with a drift time of $10.06 \mathrm{~ms}$, which is similar to that of 4-MEC (10.01 ms) and methylone (10.13 ms) as previously demonstrated in Table 12. For the confirmation of the analyte in the seized sample, the analyte was identified by the presence of the protonated molecular ion in the full scan mass spectrum. It is shown that the coupling of IMS with MS can be utilized as a confirmatory test in the qualitative analysis of seized drug samples providing additional mass spectral information.

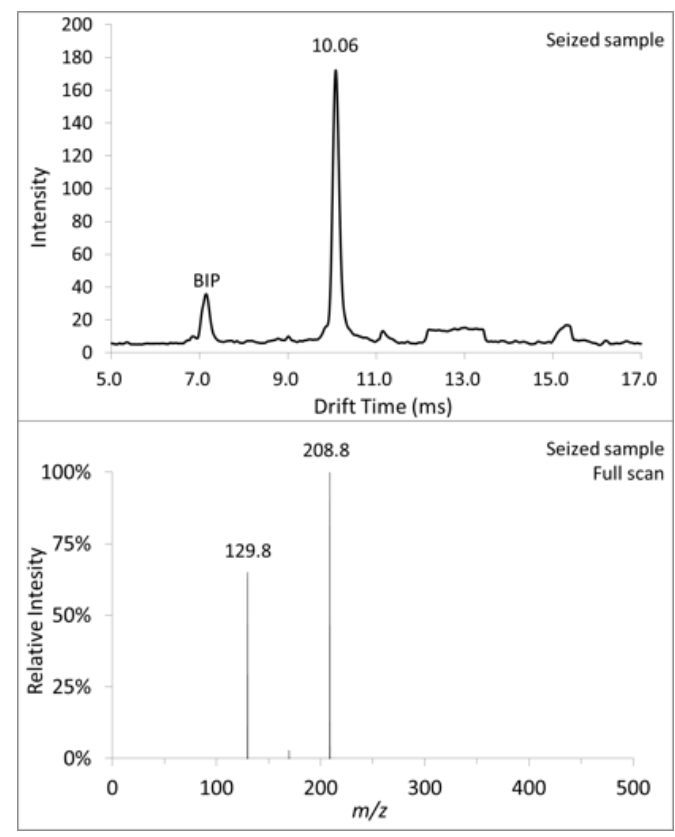

Figure 57. Ion mobility spectrum (top) and corresponding mass spectrum (bottom) of the seized drug sample by ESI-IMS-MS. The seized drug sample was found to be methylone with the presence of protonated molecular ion. 


\subsubsection{Conclusions}

The rapid detection $(20 \mathrm{~ms})$ and identification of 6 synthetic cathinones were successfully accomplished using a commercially available ESI-IMS-MS. The optimal solvent system was found to be a ratio of methanol to water 80 to $20(v / v)$ with $2.5 \%$ formic acid for the efficient protonation of all analytes. In the analysis of mixtures, the concentration of the analytes with higher proton affinity (e.g. MDPV) was reduced in order to increase the ionization of other compounds with less proton affinity in mixtures, overcoming preferential ionization. Although the baseline separation of two-analyte mixtures was achieved only for MDPV with other substances, the presence of different analytes were also confirmed in other mixtures with the observed protonated molecular ions in their mass spectra. It is important to emphasize that the coupling of IMS (Category B) with MS (Category A) can be used as a confirmatory technique, qualifying the identification criteria described in the SWGDRUG recommendation as previously mentioned in 2.2. The results from the analysis of four seized drug samples using ESIIMS-MS have shown that the ESI-IMS-MS instrument can be readily used as a confirmatory test in the qualitative analysis of synthetic cathinones with a rapid analysis time of less than five minutes. 


\section{CHAPTER 6. RAPID DETECTION AND IDENTIFICATION OF FLAKKA}

The applicability of the developed rapid screening methods is highlighted in this chapter to emphasize the usefulness of the proposed analytical techniques, IMS and DART-QTOF-MS. The rapid detection and identification capabilities of IMS and DART-QTOF-MS were evaluated in the analysis of a relatively new substance called 'Flakka'. The following sections will cover focusing on a brief introduction to the new trend of drug abuse, rapid detection and characterization of flakka by IMS, and rapid identification by DART-QTOF-MS.

\subsection{Introduction to Flakka}

A relatively new substance of abuse, 'Flakka' (also called '\$5 insanity' or 'gravel') has recently proliferated in Florida from zero cases in 2010 to 670 cases in 2014 [148149]. It is known that one Florida county toxicology laboratory reports up to 10 cases related with flakka every day in 2015. Flakka is a street name referring to alphapyrrolidinopentiophenone ( $\alpha$-PVP), a second-generation synthetic cathinone, as shown in Figure 58. The second generation of synthetic cathinones are characterized by containing a pyrrolidine group similar to 3,4-methylenedioxypyrovalerone (MDPV) and also includes, but is not limited to pyrovalerone, alpha-pyrrolidinopropiophenone ( $\alpha$-PPP), 4'methyl-alpha-pyrrolidinopropiophenone (4'-MePPP), alpha-pyrrolidinobutiophenone ( $\alpha$ PBP) and 4-methyl-alpha-pyrrolidinobutiophenone (4-MePBP) [150-151]. Since 2014, $\alpha$-PBP and $\alpha$-PVP are controlled as Schedule I substances in federal and Florida statutes $[132,152]$. 


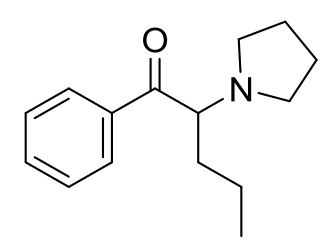

alpha-PVP

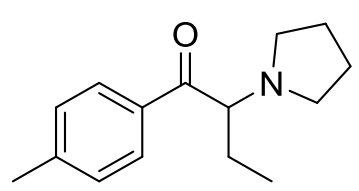

4-MePBP<smiles>CCC(C(=O)c1ccccc1)N1CCCC1</smiles>

alpha-PBP<smiles>CC(C(=O)c1ccccc1)N1CCCC1</smiles>

alpha-PPP

Figure 58. Chemical structures of alpha-PVP, 4-MePBP, alpha-PBP, and alpha-PPP.

Casale and Hays employed nuclear magnetic resonance spectroscopy (NMR), Fourier transform infrared spectroscopy (FTIR), and gas chromatography mass spectrometry (GC-MS) in the characterization and identification of $\alpha$-PVP in casework [153]. Recently, a number of toxicological studies have shown that these substances, containing the pyrrolidine group, are more potent than other stimulants, such as amphetamine and cocaine, as they block the uptake of dopamine and norepinephrine neurotransmitters $[150,154]$. In the study of rapid detection and identification capabilities, $\alpha$-PVP and its isomer, 4-MePBP, were analyzed using IMS and DARTQTOF-MS.

\subsection{Rapid Detection and Characterization by Ion Mobility Spectrometry}

Figure 59 shows overlaid ion mobility spectra of $\alpha$-PVP and 4-MePBP by the Barringer IMS. The detection of these substances was successfully achieved within 20 $\mathrm{ms}$ and the total analysis time was less than one minute from delivery of analyte to the response from the IMS instrument, the greatest advantage of the commercial Barringer IMS. As explained earlier, the drift time of the analyte is often reported as the reduced mobility, $K_{0}\left(\mathrm{~cm}^{2} / \mathrm{Vs}\right)$. The calculated $K_{0}$ for $\alpha$-PVP and 4-MePBP from our study were 1.3094 and 1.3024, respectively, as shown in Table 5. During the evaluation, the $K_{0}$ value for $\alpha$-PVP showed good agreement with the previously reported $K_{0}$ value (1.306) 
by Armenta et al. [115]. The reduced mobility of 4-MePBP has not been reported previously at the moment. The results show the potential application of IMS as a rapid detection method of controlled substances. However, false positive alarms are possible because of the similar reduced mobilities between analytes, which may limit the ${ }^{63} \mathrm{Ni}$ IMS instrument only for screening purposes.

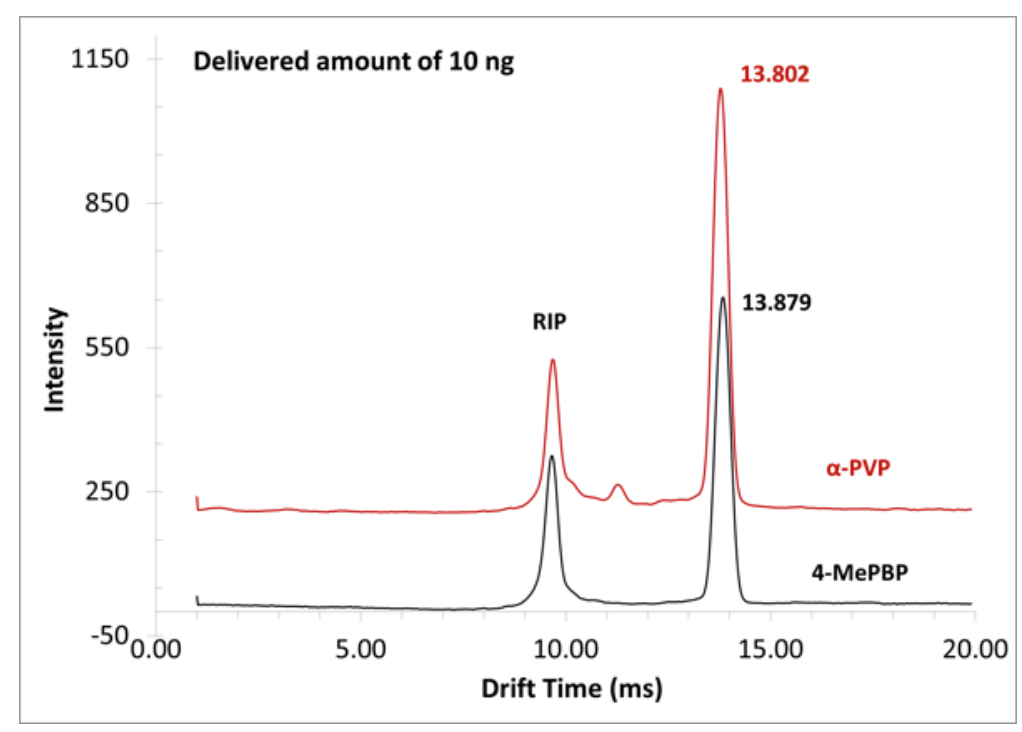

Figure 59. Overlaid ion mobility spectra of $\alpha$-PVP and 4-MePBP. Both NPS were successfully detected with its characteristic mobility and a consistent reactant ion peak (RIP).

\subsection{Rapid Identification by Direct Analysis in Real Time Mass Spectrometry}

The analytes of interest were successfully identified with high mass accuracy using DART-QTOF-MS with the presence of protonated molecular ions of each substance as shown in Figure 60. The implementation of the DART ion source enabled the rapid identification of analytes with minimal to no sample preparation and total analysis time of less than two minutes. However, constitutional isomers are not distinguished in full 
scan mode using the protonated molecular ion as the most abundant peak. Thus, the auto MS/MS mode was used and different fragmentation patterns were observed at different collision energies for $\alpha$-PVP and 4-MePBP. A series of peaks for $\alpha$-PVP including $m / z$ 91.0544, 105.0340, and 126.1278 was characteristic from the product ion scan mass spectrum at CE of $20 \mathrm{eV}$ and was compared to 4-MePBP $(\mathrm{m} / \mathrm{z}, 105.0693,112.1117$, 119.0483, and 133.1003). Therefore, the differentiation of constitutional isomers is possible with high accuracy product ion scan (MS/MS) mass spectra at CE of $20 \mathrm{eV}$.

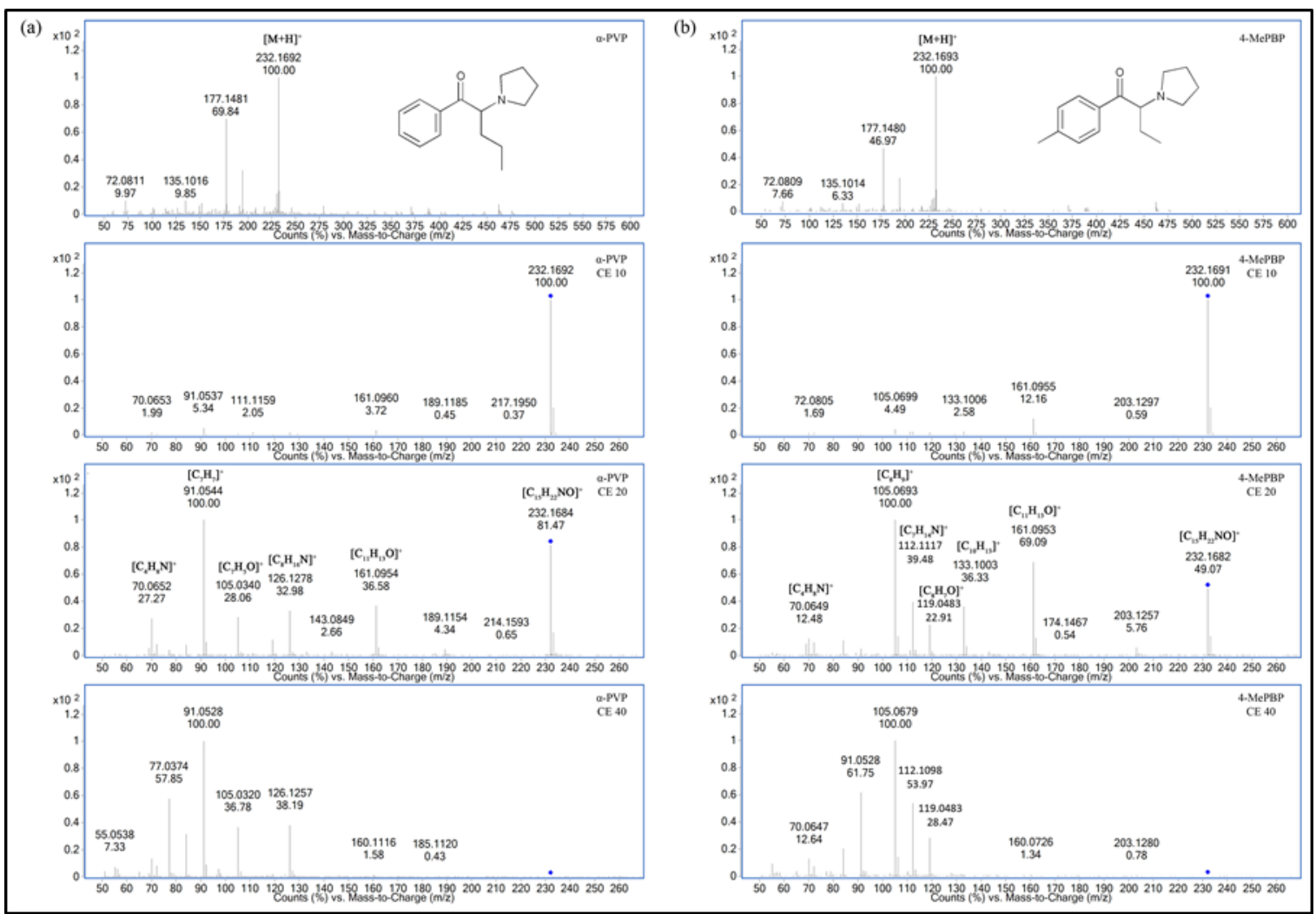

Figure 60. Full scan and product ion scan mass spectra of (a) $\alpha$-PVP and (b) 4-MePBP obtained using DART-QTOF-MS. Differentiation between two compounds can be achieved with product ion scan mass spectra at $\mathrm{CE}$ of $20 \mathrm{eV}$. 


\subsection{Conclusions}

As a proof of concept, the rapid screening of the emerging substance 'Flakka' by ${ }^{63} \mathrm{Ni}-\mathrm{IMS}$ and the identification with high mass accuracy by DART-QTOF-MS were successfully performed during the evaluation of two instruments, ${ }^{63} \mathrm{Ni}-\mathrm{IMS}$ and DARTQTOF-MS. It is shown that a product ion scan MS from DART-QTOF-MS provides a consistent relative abundance that can be searched using a library previously created using ESI ionization coupled to the same QTOF. 


\section{CHAPTER 7. DISCUSSION AND OVERALL CONCLUSIONS}

\subsection{Rapid Screening Tests for NPS by IMS and DART-QTOF-MS}

The capabilities of rapid detection and characterization have been demonstrated for the qualitative analysis of emerging new psychoactive substances (NPS) by a bench-top commercial ion mobility spectrometer, Barringer IONSCAN 400B. Ion mobility spectrometry is known as one of many well-established detection techniques for small organic compounds. A number of advantages, including ease of operation, high sensitivity, atmospheric pressure operation, and fast response, have enabled the wide usage of the portable IMS instrument in the field. The rapid detection and characterization of emerging NPS were successfully achieved with sub-nanogram detection capabilities using the Barringer IONSCAN instrument. The characteristic reduced mobilities for these substances were observed allowing the detection of each compound with high sensitivity and fast response. However, there were drawbacks in the utilization of the Barringer IONSCAN instrument, such as potential false positive alarms because of the similar reduced mobility between some compounds as well as depletion of reactant ions which may result in a small dynamic response range. Nonetheless, the Barringer IMS is an attractive alternative screening tool for the rapid detection of NPS.

The direct analysis in real time (DART) ion source coupled to mass spectrometry is a relatively new analytical technique that enables rapid analysis of compound with little to no sample preparation. The DART is a versatile ambient ionization source that can be coupled to a variety of mass spectrometers. In the application for the rapid identification of NPS, the DART source was interfaced with a high resolution quadrupole time-of-flight 
mass spectrometer (QTOF-MS). The uniqueness of DART-QTOF-MS is the production of protonated molecular ion in positive mode with high mass accuracy. In addition, the ability of product ions scan through the auto MS/MS and targeted MS/MS modes enables the structural elucidation of compounds and differentiation of constitutional isomers. To evaluate figures of merit from both Barringer IMS and DART-QTOF-MS, the seized drugs samples provided by a local forensic laboratory were analyzed and the results showed good agreement. The findings from these two techniques have been published in a peer-reviewed journal.

\subsection{Confirmatory Techniques for NPS by GC-MS and ESI-IMS-MS}

Gas chromatography mass spectrometry (GC-MS) is the gold standard technique that is widely used in the forensic laboratories for various applications. While GC-MS equipped with a single quadrupole mass spectrometer and an electron ionization (EI) source is the most commonly utilized setting, a GC system coupled with a triple quadrupole mass spectrometer (MS/MS or QqQ) was utilized in the qualitative analysis of emerging NPS proposing as a new confirmatory test. The novelty of this GC-MS/MS is the capability of multiple transitions scan known as multiple reaction monitoring (MRM) mode. With the MRM acquisition mode, only specific transitions can be monitored eliminating noises, background peaks, or other co-eluting analyte peaks. In addition, it is shown that additional spectral information from product ion scan and MRM can be used to differentiate isomers, which is one of major challenges in the analysis of NPS. Most importantly, the unambiguous identification of NPS was successfully achieved with the implementation of the chemical ionization (CI) source for those 
substances that are extensively fragmented in EI mass spectra. These results also have been reported and published in peer reviewed literature. The GC system coupled to a high resolution QTOF mass spectrometer also has been proposed for an alternative confirmatory technique in the analysis of NPS. The hyphenated analytical technique, GC-QTOF-MS, was developed to provide advantages to both GC-MS/MS and LCQTOF-MS. The MS/MS capability from GC-MS/MS was enhanced switching the third quadrupole with a TOF mass analyzer. As a result, high resolution full scan and MS/MS scan with a high scan rate was possible with enhanced resolving power in GC-QTOFMS. To investigate the potential of GC-QTOF-MS as a confirmatory method, various NPS have been analyzed in both EI and CI mode. From the preliminary results, it is expected that the creation of a database using the acquired high mass accuracy full scan and MS/MS scan mass spectra will be beneficial in various aspects.

A total number of six synthetic cathinones were also analyzed using a commercial ESI-IMS-MS (Excellims RA4100). This hybrid analytical technique consists of an ion mobility spectrometer (IMS) unit with an electrospray ionization (ESI) source and a single quadrupole mass analyzer. The coupling of a gas-phase separation technique (IMS) to mass spectrometry is a competitive candidate as an alternative confirmatory method in drug analysis, especially for controlled substances, regarding the SWGDRUG guidelines. With the development of an optimal solvent system for the ESI process, the capabilities of rapid detection and identification would provide low cost analysis for NPS in forensic laboratories. 
Table 13. Comparison of various analytical techniques evaluated in this dissertation.

\begin{tabular}{|c|c|c|c|c|c|}
\hline & ${ }^{63} \mathrm{Ni}-\mathrm{IMS}$ & DART-QTOF & GC-MS/MS & GC-QTOF & ESI-IMS-MS \\
\hline & \multicolumn{2}{|c|}{ Screening techniques } & \multicolumn{3}{|c|}{ Confirmatory techniques } \\
\hline 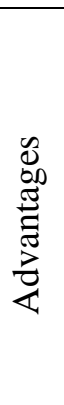 & $\begin{array}{ll}\text { - } & \text { Inexpensive } \\
\text { analysis } \\
\text { - High } \\
\text { sensitivity } \\
\text { - Fast } \\
\text { detection } \\
\text { - Easy } \\
\text { operation \& } \\
\text { maintenance }\end{array}$ & $\begin{array}{l}\text { - Min. sample } \\
\text { preparation } \\
\text { - Easy } \\
\text { operation \& } \\
\text { maintenance } \\
\text { - MS/MS } \\
\text { capability }\end{array}$ & $\begin{array}{ll}\text { - } & \text { Gold } \\
& \text { standard } \\
\text { - } & \text { High } \\
& \text { sensitivity } \\
\text { - } & \text { MS/MS } \\
& \text { capability }\end{array}$ & $\begin{array}{ll}\text { - } & \text { High } \\
\text { sensitivity } \\
\text { - } & \text { High mass } \\
\text { accuracy } \\
\text { - } & \text { High } \\
\text { versatility } \\
\text { - } \\
\text { MS/MS } \\
\text { capability }\end{array}$ & $\begin{array}{ll}- & \text { Fast } \\
\text { confirmation } \\
\text { - Inexpensive } \\
\text { analysis }\end{array}$ \\
\hline 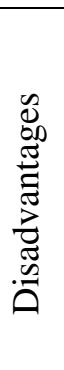 & $\begin{array}{ll}\text { - } & \text { No } \\
\text { identification } \\
\text { - Small } \\
\text { dynamic } \\
\text { range } \\
\text { - Semi- } \\
\text { quantitative } \\
\text { analysis }\end{array}$ & $\begin{array}{l}\text { - } \text { No } \\
\text { separation } \\
\text { capability } \\
\text { - Limited } \\
\text { ionization } \\
\text { capability }\end{array}$ & $\begin{array}{ll} & \text { Expensive } \\
\text { instrument } \\
\text { - } & \text { Time } \\
\text { consuming } \\
\text { analysis }\end{array}$ & 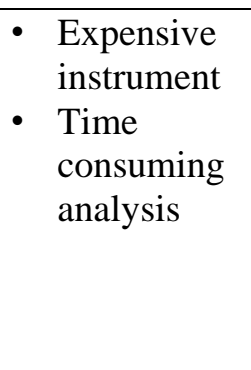 & $\begin{array}{ll}\text { - } & \text { Novel } \\
\text { technique } \\
\text { - Incomplete } \\
\text { separation }\end{array}$ \\
\hline తి & $\sim 80 \mathrm{pg}$ & $\sim 340 \mathrm{pg}$ & $\begin{array}{c}\sim 350 \mathrm{pg} \\
(\mathrm{EI}, \mathrm{MRM}) \\
\sim 750 \mathrm{pg} \\
(\mathrm{CI}, \mathrm{MRM})\end{array}$ & $\begin{array}{c}\sim 370 \mathrm{pg} \\
(\mathrm{EI}, \text { Full scan })\end{array}$ & $19 \mathrm{ppm}$ \\
\hline
\end{tabular}

\subsection{Concluding Remarks}

This dissertation evaluates various analytical techniques for the qualitative analysis of emerging new psychoactive substances (NPS), commonly referred to as designer drugs or legal highs, at different levels. It has been demonstrated that each analytical technique has advantages and disadvantages in terms of costs, the degree of separation, the limit of detection, and the identification capability as shown in Table 13. Regardless, the capabilities of rapid detection and characterization in the analysis of NPS by IMS is beneficial as it opens up the potential of on-site analysis by portable IMS. In addition, 
the application of DART-QTOF-MS in the analysis of controlled substances will facilitate the identification of compounds with high throughput and high-speed capabilities. As a well-established analytical technique, GC-MS is the gold standard analytical method in most forensic laboratories. However, the traditional GC-MS with a single quadrupole mass analyzer may face analytical challenges, including the complexity of analytes and matrices as well as the presence of isomers. In the case of the isomer separation and analyte isolation from complex matrices, state-of-the-art instruments, such as GC-MS/MS and GC-QTOF-MS, can provide a solution with different types of scanning modes. These instruments will be more common in forensic laboratories as they are becoming more popular and affordable. Finally, the hybrid analytical technique, ESI-IMS-MS, would provide high speed and low cost analysis for the separation and identification of compound. 


\section{REFERENCES}

[1] COUNCIL DECISION 2005/387/JHA of 10 May 2005 on the information exchange, risk-assessment and control of new psychoactive substances, Official Journal of the European Union, 2005, L 127, 20.5.2005, 32-37.

[2] United Nations Office of Drugs and Crime (UNODC) (2013). The challenge of new psychoactive substances. Vienna, Austria. Available at:

http://www.unodc.org/unodc/en/scientists/the-challenge-of-new-psychoactivesubstances---global-smart-programme.html.

[3] United Nations Office of Drugs and Crime (UNODC) (2013). World Drug Report 2013. Vienna, Austria. Available at: http://www.unodc.org/wdr2013/.

[4] Brandt, S.D., King, L.A., Evans-Brown, M. (2014). The new drug phenomenon. Drug Testing and Analysis, 6 (7-8): 587-597.

[5] Levine, B. (2003). Principles of forensic toxicology, American Association for Clinical Chemistry.

[6] Shulgin, A., Shulgin, A. (1991). PiHKAL: A Chemical Love story, Transform Press, Berkeley, California.

[7] Shulgin, A., Shulgin, A. (1997). TiHKAL: The Continuation, Transform Press, Berkeley, California.

[8] United Nations Office of Drugs and Crime (UNODC) (2014). World Drug Report 2014. Vienna, Austria. Available at: http://www.unodc.org/wdr2014/.

[9] United Nations Office of Drugs and Crime (UNODC) (2015). World Drug Report 2015. Vienna, Austria. Available at: http://www.unodc.org/wdr2015/.

[10] American Association of Poison Control Centers (AAPCC) (2015). Bath Salts Data. Alexandria, Virginia, USA. Available at: http://www.aapcc.org/alerts/bath-salts [June 29 2015]. 
[11] American Association of Poison Control Centers (AAPCC) (2015). Synthetic Marijuana Data. Alexandria, Virginia, USA. Available at: http://www.aapcc.org/alerts/synthetic-marijuana [June 29 2015].

[12] Newberry, J., Wodak, A., Sellman, D., Robinson, G. (2014). New Zealand's regulation of new psychoactive substances. BMJ, 348.

[13] Uchiyama, N., Matsuda, S., Kawamura, M., Kikura-Hanajiri, R., Goda, Y. (2014). Identification of two new-type designer drugs, piperazine derivative MT-45 (I-C6) and synthetic peptide Noopept (GVS-111), with synthetic cannabinoid A-834735, cathinone derivative 4-methoxy- $\alpha$-PVP, and phenethylamine derivative 4-methylbuphedrine from illegal products. Forensic Toxicology, 32 (1): 9-18.

[14] Boulanger-Gobeil, C., St-Onge, M., Laliberté, M., Auger, P.L. (2012). Seizures and hyponatremia related to ethcathinone and methylone poisoning. Journal of Medical Toxicology, 8 (1): 59-61.

[15] Carbone, P.N., Carbone, D.L., Carstairs, S.D., Luzi, S.A. (2013). Sudden Cardiac Death Associated With Methylone Use. The American Journal of Forensic Medicine and Pathology, 34 (1): 26-28.

[16] Khullar, V., Jain, A., Sattari, M. (2014). Emergence of New Classes of Recreational Drugs-Synthetic Cannabinoids and Cathinones. Journal of General Internal Medicine, 15.

[17] Smith, F. (2004). Handbook of forensic drug analysis, Academic Press.

[18] Zaitsu, K., Katagi, M., Kamata, H., Miki, A., Tsuchihashi, H. (2008). Discrimination and identification of regioisomeric $\beta$-keto analogues of 3,4methylenedioxyamphetamines by gas chromatography-mass spectrometry. Forensic Toxicology, 26 (2): 45-51.

[19] Zaitsu, K., Katagi, M., Kamata, H.T., Kamata, T., Shima, N., Miki, A., Tsuchihashi, H., Mori, Y. (2009). Determination of the metabolites of the new designer drugs bkMBDB and bk-MDEA in human urine. Forensic Science International, 188 (1): 131-139. 
[20] Westphal, F., Junge, T. (2012). Ring positional differentiation of isomeric Nalkylated fluorocathinones by gas chromatography/tandem mass spectrometry. Forensic Science International, 223 (1-3): 97-105.

[21] Westphal, F., Sönnichsen, F.D., Thiemt, S. (2012). Identification of 1-butyl-3-(1-(4methyl)naphthoyl)indole in a herbal mixture. Forensic Science International, 215 (1-3): 8-13.

[22] Zaitsu, K., Miyagawa, H., Sakamoto, Y., Matsuta, S., Tsuboi, K., Nishioka, H., Katagi, M., Sato, T., Tatsuno, M., Tsuchihashi, H., Suzuki, K., Ishii, A. (2013). Mass spectrometric differentiation of the isomers of mono-methoxyethylamphetamines and mono-methoxydimethylamphetamines by GC-EI-MS-MS. Forensic Toxicology, 31 (2): 292-300.

[23] Nakazono, Y., Tsujikawa, K., Kuwayama, K., Kanamori, T., Iwata, Y., Miyamoto, K., Kasuya, F., Inoue, H. (2013). Differentiation of regioisomeric fluoroamphetamine analogs by gas chromatography-mass spectrometry and liquid chromatography-tandem mass spectrometry. Forensic Toxicology, 31 (2): 241-250.

[24] Wohlfarth, A., Weinmann, W., Dresen, S. (2010). LC-MS/MS screening method for designer amphetamines, tryptamines, and piperazines in serum. Analytical and Bioanalytical Chemistry, 396 (7): 2403-2414.

[25] Sørensen, L.K. (2011). Determination of cathinones and related ephedrines in forensic whole-blood samples by liquid-chromatography-electrospray tandem mass spectrometry. Journal of Chromatography B, 879 (11-12): 727-736.

[26] Dresen, S., Kneisel, S., Weinmann, W., Zimmermann, R., Auwärter, V. (2011). Development and validation of a liquid chromatography-tandem mass spectrometry method for the quantitation of synthetic cannabinoids of the aminoalkylindole type and methanandamide in serum and its application to forensic samples. Journal of Mass Spectrometry, 46 (2): 163-171.

[27] Swortwood, M.J., Boland, D.M., DeCaprio, A.P. (2013). Determination of 32 cathinone derivatives and other designer drugs in serum by comprehensive LC-QQQMS/MS analysis. Analytical and Bioanalytical Chemistry, 405 (4): 1383-1397. 
[28] Swortwood, M.J., Hearn, W.L., DeCaprio, A.P. (2014). Cross-reactivity of designer drugs, including cathinone derivatives, in commercial enzyme-linked immunosorbent assays. Drug Testing and Analysis, 6 (7-8): 716-727.

[29] Westphal, F., Rösner, P., Junge, T. (2010). Differentiation of regioisomeric ringsubstituted fluorophenethylamines with product ion spectrometry. Forensic Science International, 194 (1-3): 53-59.

[30] Musah, R.A., Domin, M.A., Cody, R.B., Lesiak, A.D., John Dane, A., Shepard, J.R. (2012). Direct analysis in real time mass spectrometry with collision-induced dissociation for structural analysis of synthetic cannabinoids. Rapid Communications in Mass Spectrometry, 26 (19): 2335-2342.

[31] Lesiak, A.D., Musah, R.A., Cody, R.B., Domin, M.A., Dane, A.J., Shepard, J.R. (2013). Direct analysis in real time mass spectrometry (DART-MS) of "bath salt" cathinone drug mixtures. Analyst, 138 (12): 3424-3432.

[32] Wu, C., Siems, W.F., Hill, H.H. (2000). Secondary Electrospray Ionization Ion Mobility Spectrometry/Mass Spectrometry of Illicit Drugs. Analytical chemistry, 72 (2): 396-403.

[33] Matz, L.M., Hill, H.H. (2001). Evaluation of Opiate Separation by High-Resolution Electrospray Ionization-Ion Mobility Spectrometry/Mass Spectrometry. Analytical chemistry, 73 (8): 1664-1669.

[34] Matz, L.M., Hill, H.H. (2002). Evaluating the Separation of Amphetamines by Electrospray Ionization Ion Mobility Spectrometry/MS and Charge Competition within the ESI Process. Analytical chemistry, 74 (2): 420-427.

[35] Holness, H., Almirall, J. (2013). Speciation effects of solvent chemistry on the analysis of drugs and explosives by electrospray ion mobility mass spectrometry. International Journal for Ion Mobility Spectrometry, 16 237-246.

[36] Dunham, S.J.B., Hooker, P.D., Hyde, R.M. (2012). Identification, extraction and quantification of the synthetic cannabinoid JWH-018 from commercially available herbal marijuana alternatives. Forensic Science International, 223 (1-3): 241-244. 
[37] Seely, K.A., Lapoint, J., Moran, J.H., Fattore, L. (2012). Spice drugs are more than harmless herbal blends: A review of the pharmacology and toxicology of synthetic cannabinoids. Prog Neuro-Psychoph, 39 (2): 234-243.

[38] Musah, R.A., Domin, M.A., Walling, M.A., Shepard, J.R. (2012). Rapid identification of synthetic cannabinoids in herbal samples via direct analysis in real time mass spectrometry. Rapid Communications in Mass Spectrometry, 26 (9): 1109-1114.

[39] Huffman, J.W., Dai, D., Martin, B.R., Compton, D.R. (1994). Design, Synthesis and Pharmacology of Cannabimimetic Indoles. Bioorganic \& Medicinal Chemistry Letters, 4 (4): 563-566.

[40] Bononi, M., Belgi, P., Tateo, F. (2011). Analytical data for identification of the cannabimimetic phenylacetylindole JWH-203. Journal of analytical toxicology, 35 (6): $360-363$.

[41] McClean, J.M., Anspikian, A., Tsuang, J.W. (2012). Bath Salt Use: A Case Report and Review of the Literature. Journal of Dual Diagnosis, 8 (3): 250-256.

[42] Prosser, J., Nelson, L. (2012). The Toxicology of Bath Salts: A Review of Synthetic Cathinones. Journal of Medical Toxicology, 8 (1): 33-42.

[43] Airuehia, E., Walker, L.Y., Nittler, J. (2012). A Review of 'Bath Salts': Evolving Designer Drugs of Abuse. Journal of Clinical Toxicology, 2 (4): 125.

[44] Gibbons, S., Zloh, M. (2010). An analysis of the 'legal high' mephedrone. Bioorganic and Medicinal Chemistry Letters, 20 (14): 4135-4139.

[45] Watterson, L.R., Burrows, B.T., Hernandez, R.D., Moore, K.N., Grabenauer, M., Marusich, J.A., Olive, M.F. (2015). Effects of $\alpha$ - Pyrrolidinopentiophenone and 4Methyl-N-Ethylcathinone, Two Synthetic Cathinones Commonly Found in SecondGeneration "Bath Salts," on Intracranial Self-Stimulation Thresholds in Rats. International Journal of Neuropsychopharmacology, 18 (1): pyu014.

[46] Fantegrossi, W.E., Harrington, A.W., Eckler, J.R., Arshad, S., Rabin, R.A., Winter, J.C., Coop, A., Rice, K.C., Woods, J.H. (2005). Hallucinogen-like actions of 2, 5- 
dimethoxy-4-(n)-propylthiophenethylamine (2C-T-7) in mice and rats. Psychopharmacology, 181 (3): 496-503.

[47] da Costa, J.L., Wang, A.Y., Micke, G.A., Maldaner, A.O., Romano, R.L., MartinsJúnior, H.A., Neto, O.N., Tavares, M.F.M. (2007). Chemical identification of 2, 5dimethoxy-4-bromoamphetamine (DOB). Forensic Science International, 173 (2): 130136.

[48] Moya, P.R., Berg, K.A., Gutiérrez-Hernandez, M.A., Sáez-Briones, P., ReyesParada, M., Cassels, B.K., Clarke, W.P. (2007). Functional selectivity of hallucinogenic phenethylamine and phenylisopropylamine derivatives at human 5-hydroxytryptamine (5-HT) 2A and 5-HT2C receptors. Journal of Pharmacology and Experimental Therapeutics, 321 (3): 1054-1061.

[49] Nichols, D.E., Frescas, S.P., Chemel, B.R., Rehder, K.S., Zhong, D., Lewin, A.H. (2008). High specific activity tritium-labeled N-(2-methoxybenzyl)-2, 5-dimethoxy-4iodophenethylamine (INBMeO): a high-affinity 5-HT 2 A receptor-selective agonist radioligand. Bioorganic \& medicinal chemistry, 16 (11): 6116-6123.

[50] McGrane, O. (2011). Alarming Trends in a Novel Class of Designer Drugs. Journal of Clinical Toxicology, 1 (2).

[51] Haroz, R., Greenberg, M.I. (2006). New Drugs of Abuse in North America. Clinics in Laboratory Medicine, 26 (1): 147-164.

[52] Rickli, A., Luethi, D., Reinisch, J., Buchy, D., Hoener, M.C., Liechti, M.E. (2015). Receptor interaction profiles of novel N-2-methoxybenzyl (NBOMe) derivatives of 2,5dimethoxy-substituted phenethylamines (2C drugs). Neuropharmacology, 99 546-553.

[53] Zawilska, J.B. (2014). Methoxetamine - a novel recreational drug with potent hallucinogenic properties. Toxicology Letters, 230 (3): 402-407.

[54] Hondebrink, L., Nugteren-van Lonkhuyzen, J.J., Van Der Gouwe, D., Brunt, T.M. (2015). Monitoring new psychoactive substances (NPS) in The Netherlands: Data from the drug market and the Poisons Information Centre. Drug and Alcohol Dependence, 147 (0): 109-115. 
[55] Corazza, O., Assi, S., Schifano, F. (2013). From "Special K" to "Special M": The Evolution of the Recreational Use of Ketamine and Methoxetamine. CNS Neuroscience \& Therapeutics, 19 (6): 454-460.

[56] Meyer, M., Bach, M., Welter, J., Bovens, M., Turcant, A., Maurer, H. (2013). Ketamine-derived designer drug methoxetamine: metabolism including isoenzyme kinetics and toxicological detectability using GC-MS and LC-(HR-)MS n. Analytical and Bioanalytical Chemistry, 405 (19): 6307-6321.

[57] States, U. Title 21 United States Code (USC) Controlled Substances Act. U.S., 2012.

[58] Meyer, M.R., Caspar, A., Brandt, S.D., Maurer, H.H. (2013). A qualitative/quantitative approach for the detection of 37 tryptamine-derived designer drugs, $5 \beta$-carbolines, ibogaine, and yohimbine in human urine and plasma using standard urine screening and multi-analyte approaches. Analytical and Bioanalytical Chemistry, 113.

[59] Rosenbaum, C.D., Carreiro, S.P., Babu, K.M. (2012). Here today, gone tomorrow... and back again? A review of herbal marijuana alternatives (K2, Spice), synthetic cathinones (bath salts), kratom, Salvia divinorum, methoxetamine, and piperazines. Journal of Medical Toxicology, 8 (1): 15-32.

[60] The Scientific Working Group for the Analysis of Seized Drugs (SWGDRUG) Recommendations. http://www.swgdrug.org/approved.htm (accessed February 24).

[61] Alaska Department of Public Safety (2014). Controlled Substances Analysis Manual. Available at: http://dps.alaska.gov/CrimeLab/docs/QA/.

[62] Virginia Department of Forensic Science (2014). Controlled Substances Procedures Manual. Available at: http://www.dfs.virginia.gov/.

[63] Virginia Department of Forensic Science (2014). Controlled Substances Instructions for the AccuTOF-DART SVP. Available at: http://www.dfs.virginia.gov/ [February 7, 2014].

[64] Robards, K., Haddad, P.R., Jackson, P.E. (1994). Principles and practice of modern chromatographic methods, Academic Press. 
[65] Kitson, F.G., Larsen, B.S., McEwen, C.N. (1996). Gas chromatography and mass spectrometry: a practical guide, Academic Press.

[66] Sparkman, O.D., Penton, Z., Kitson, F.G. (2011). Gas Chromatography and Mass Spectrometry: A Practical Guide: A Practical Guide, Academic Press.

[67] Eiceman, G.A., Karpas, Z. (2005). Ion Mobility Spectrometry, 2nd ed. CRC Press, London, UK.

[68] Smiths Detection. Ion Mobility Spectrometry (IMS). http://www.smithsdetection.com/index.php/technologies/ion-mobility-spectrometry.html (accessed September 29 2014).

[69] Borsdorf, H., Eiceman, G.A. (2006). Ion Mobility Spectrometry: Principles and Applications. Appl Spectrosc Rev, 41 (4): 323-375.

[70] Kaur-Atwal, G., O’Connor, G., Aksenov, A.A., Bocos-Bintintan, V., Thomas, C.P., Creaser, C.S. (2009). Chemical standards for ion mobility spectrometry: a review. International Journal for Ion Mobility Spectrometry, 12 (1): 1-14.

[71] Karasek, F.W., Karasek, D.E., Kim, S.H. (1975). Detection of lysergic acid diethylamide, $\Delta 9$-tetrahydrocannabinol and related compounds by plasma chromatography. Journal of Chromatography A, 105 (2): 345-352.

[72] Karasek, F.W., Hill Jr, H.H., Kim, S.H. (1976). Plasma chromatography of heroin and cocaine with mass-identified mobility spectra. Journal of Chromatography A, 117 (2): 327-336.

[73] Keller, T., Miki, A., Regenscheit, P., Dirnhofer, R., Schneider, A., Tsuchihashi, H. (1998). Detection of designer drugs in human hair by ion mobility spectrometry (IMS). Forensic Science International, 94 (1-2): 55-63.

[74] Lai, H., Corbin, I., Almirall, J. (2008). Headspace sampling and detection of cocaine, MDMA, and marijuana via volatile markers in the presence of potential interferences by solid phase microextraction-ion mobility spectrometry (SPME-IMS). Analytical and Bioanalytical Chemistry, 392 (1): 105-113. 
[75] Miki, A., Keller, T., Regenscheit, P., Dirnhofer, R., Tatsuno, M., Katagi, M., Nishikawa, M., Tsuchihashi, H. (1997). Application of ion mobility spectrometry to the rapid screening of methamphetamine incorporated in hair. Journal of Chromatography B: Biomedical Sciences and Applications, 692 (2): 319-328.

[76] Wu, C., Siems, W.F., Asbury, G.R., Hill, H.H. (1998). Electrospray Ionization HighResolution Ion Mobility Spectrometry-Mass Spectrometry. Analytical chemistry, 70 (23): 4929-4938.

[77] Keller, T., Keller, A., Tutsch-Bauer, E., Monticelli, F. (2006). Application of ion mobility spectrometry in cases of forensic interest. Forensic Science International, 161 (2-3): 130-140.

[78] Asbury, G.R., Klasmeier, J., Hill Jr, H.H. (2000). Analysis of explosives using electrospray ionization/ion mobility spectrometry (ESI/IMS). Talanta, 50 (6): 1291-1298.

[79] Bohrer, B.C., Merenbloom, S.I., Koeniger, S.L., Hilderbrand, A.E., Clemmer, D.E. (2008). Biomolecule analysis by ion mobility spectrometry. Annual Review of Analytical Chemistry, $1293-327$.

[80] Harvey, S.R., MacPhee, C.E., Barran, P.E. (2011). Ion mobility mass spectrometry for peptide analysis. Methods, 54 (4): 454-461.

[81] Kanu, A.B., Wu, C., Hill Jr, H.H. (2008). Rapid preseparation of interferences for ion mobility spectrometry. Analytica Chimica Acta, 610 (1): 125-134.

[82] Hunka, D.E., Austin, D.E. (2006). Ion mobility spectrometer/mass spectrometer (IMS-MS). Sandia National Laboratories, Albuquerque, NM, Tech. Rep. SAND20056908, 1-36.

[83] Skoog, D., Holler, F., Crouch, S. (2007). Principles of Instrumental Analysis Sixth Edition, Thomson Brooks/Cole.

[84] de Hoffmann, E., Stroobant, V. (2007). Mass Spectrometry:Principles and Applications, 3rd Wiley 
[85] Domin, M., Cody, R. (2014). Ambient Ionization Mass Spectrometry, Royal Society of Chemistry.

[86] Fenn, J., Mann, M., Meng, C., Wong, S., Whitehouse, C. (1989). Electrospray ionization for mass spectrometry of large biomolecules. Science, 246 (4926): 64-71.

[87] McLafferty, F. (1981). Tandem mass spectrometry. Science, 214 (4518): 280-287.

[88] Uhl, M. (1997). Determination of drugs in hair using GC/MS/MS. Forensic Science International, 84 (1-3): 281-294.

[89] Kintz, P., Cirimele, V., Jamey, C., Ludes, B. (2003). Testing for GHB in hair by GC/MS/MS after a single exposure application to document sexual assault. Journal of forensic sciences, 48 (1): 195-200.

[90] Jankovics, P., Váradi, A., Tölgyesi, L., Lohner, S., Németh-Palotás, J., KőszegiSzalai, H. (2011). Identification and characterization of the new designer drug $4^{\prime}-$ methylethcathinone (4-MEC) and elaboration of a novel liquid chromatography-tandem mass spectrometry (LC-MS/MS) screening method for seven different methcathinone analogs. Forensic Science International, 210 (1-3): 213-220.

[91] Chernushevich, I.V., Loboda, A.V., Thomson, B.A. (2001). An introduction to quadrupole-time-of-flight mass spectrometry. Journal of Mass Spectrometry, 36 (8): 849865.

[92] Agilent Technologies. Agilent 6200 Series TOF and 6500 Series Q-TOF LC/MS System Concepts Guide. http://www.chem.agilent.com/Library/usermanuals/Public/G3335-90142_TOF_QTOF_Concepts.pdf (accessed April 29 2015).

[93] Ferrer, I., Thurman, E.M. (2010). Identification of a New Antidepressant and its Glucuronide Metabolite in Water Samples Using Liquid Chromatography/Quadrupole Time-of-Flight Mass Spectrometry. Analytical chemistry, 82 (19): 8161-8168.

[94] Ferrer, I., Thurman, E.M. (2012). Analysis of 100 pharmaceuticals and their degradates in water samples by liquid chromatography/quadrupole time-of-flight mass spectrometry. Journal of Chromatography A, 1259 (0): 148-157. 
[95] Fornal, E., Stachniuk, A., Wojtyla, A. (2013). LC-Q/TOF mass spectrometry data driven identification and spectroscopic characterisation of a new 3,4-methylenedioxy-Nbenzyl cathinone (BMDP). Journal of Pharmaceutical and Biomedical Analysis, 72 (0): 139-144.

[96] Fornal, E. (2014). Study of collision-induced dissociation of electrospray-generated protonated cathinones. Drug Testing and Analysis, 6 (7-8): 705-715.

[97] Cody, R.B., Laramée, J.A., Durst, H.D. (2005). Versatile new ion source for the analysis of materials in open air under ambient conditions. Analytical chemistry, 77 (8): 2297-2302.

[98] Lesiak, A.D., Shepard, J.R. (2014). Recent advances in forensic drug analysis by DART-MS. Bioanalysis, 6 (6): 819-842.

[99] Cody, R.B. (2009). Observation of Molecular Ions and Analysis of Nonpolar Compounds with the Direct Analysis in Real Time Ion Source. Analytical chemistry, 81 (3): 1101-1107.

[100] Gross, J. (2014). Direct analysis in real time-a critical review on DART-MS. Analytical and Bioanalytical Chemistry, 406 (1): 63-80.

[101] Vaclavik, L., Cajka, T., Hrbek, V., Hajslova, J. (2009). Ambient mass spectrometry employing direct analysis in real time (DART) ion source for olive oil quality and authenticity assessment. Analytica Chimica Acta, 645 (1-2): 56-63.

[102] Kim, H.J., Jang, Y.P. (2009). Direct analysis of curcumin in turmeric by DARTMS. Phytochemical Analysis, 20 (5): 372-377.

[103] Kawamura, M., Kikura-Hanajiri, R., Goda, Y. (2010). Simple and rapid screening for methamphetamine and 3, 4-methylenedioxymethamphetamine (MDMA) and their metabolites in urine using direct analysis in real time (DART)-TOFMS. Yakugaku zasshi: Journal of the Pharmaceutical Society of Japan, 131 (5): 827-833.

[104] Doué, M., Dervilly-Pinel, G., Pouponneau, K., Monteau, F., Le Bizec, B. (2014). Direct analysis in real time-high resolution mass spectrometry (DART-HRMS): a high 
throughput strategy for identification and quantification of anabolic steroid esters. Drug Testing and Analysis.

[105] Nilles, J.M., Connell, T.R., Durst, H.D. (2009). Quantitation of Chemical Warfare Agents Using the Direct Analysis in Real Time (DART) Technique. Analytical chemistry, 81 (16): 6744-6749.

[106] Harris, G.A., Kwasnik, M., Fernández, F.M. (2011). Direct analysis in real time coupled to multiplexed drift tube ion mobility spectrometry for detecting toxic chemicals. Analytical chemistry, 83 (6): 1908-1915.

[107] Grange, A.H., Sovocool, G.W. (2011). Detection of illicit drugs on surfaces using direct analysis in real time (DART) time-of-flight mass spectrometry. Rapid Communications in Mass Spectrometry, 25 (9): 1271-1281.

[108] Grange, A.H. (2013). Semi - quantitative analysis of contaminants in soils by direct analysis in real time (DART) mass spectrometry. Rapid Communications in Mass Spectrometry, 27 (2): 305-318.

[109] Steiner, R.R., Larson, R.L. (2009). Validation of the direct analysis in real time source for use in forensic drug screening. Journal of forensic sciences, 54 (3): 617-622.

[110] Chernetsova, E.S., Morlock, G.E. (2011). Determination of drugs and drug-like compounds in different samples with direct analysis in real time mass spectrometry. Mass Spectrometry Reviews, 30 (5): 875-883.

[111] Duvivier, W.F., van Beek, T.A., Pennings, E.J.M., Nielen, M.W.F. (2014). Rapid analysis of $\Delta$-9-tetrahydrocannabinol in hair using direct analysis in real time ambient ionization orbitrap mass spectrometry. Rapid Communications in Mass Spectrometry, 28 (7): 682-690.

[112] Howlett, S.E., Steiner, R.R. (2011). Validation of Thin Layer Chromatography with AccuTOF-DART TM Detection for Forensic Drug Analysis. Journal of forensic sciences, 56 (5): 1261-1267. 
[113] Lesiak, A.D., Musah, R.A., Domin, M.A., Shepard, J.R.E. (2014). DART-MS as a Preliminary Screening Method for "Herbal Incense": Chemical Analysis of Synthetic Cannabinoids. Journal of forensic sciences, 59 (2): 337-343.

[114] Fytche, L.M., Hupé, M., Kovar, J.B., Pilon, P. (1992). Ion mobility spectrometry of drugs of abuse in customs scenarios: concentration and temperature study. Journal of forensic sciences, 37 (6): 1550-1566.

[115] Armenta, S., Garrigues, S., de la Guardia, M., Brassier, J., Alcalà, M., Blanco, M., Perez-Alfonso, C., Galipienso, N. (2014). Detection and characterization of emerging psychoactive substances by ion mobility spectrometry. Drug Testing and Analysis, 7 (4): 280-289.

[116] Joshi, M., Cetroni, B., Camacho, A., Krueger, C., Midey, A.J. (2014). Analysis of synthetic cathinones and associated psychoactive substances by ion mobility spectrometry. Forensic Science International, 244 (0): 196-206.

[117] Sysoev, A.A., Poteshin, S.S., Chernyshev, D.M., Karpov, A.V., Tuzkov, Y.B., Kyzmin, V.V., Sysoev, A.A. (2014). Analysis of New Synthetic Drugs by Ion Mobility Time-of-Flight Mass Spectrometry. European Journal of Mass Spectrometry, 20 (2): 185192.

[118] Kanu, A.B., Brandt, S.D., Williams, M.D., Zhang, N., Hill, H.H. (2013). Analysis of psychoactive cathinones and tryptamines by electrospray ionization atmospheric pressure ion mobility time-of-flight mass spectrometry. Analytical chemistry, 85 (18): 8535-8542.

[119] Gwak, S., Almirall, J.R. (2015). Rapid screening of 35 new psychoactive substances by ion mobility spectrometry (IMS) and direct analysis in real time (DART) coupled to quadrupole time-of-flight mass spectrometry (QTOF-MS). Drug Testing and Analysis, Ahead of Print.

[120] Miller, J.N., Miller, J.C. (2010). Statistics and chemometrics for analytical chemistry, 6th Pearson Education, New Jersey, USA.

[121] Holness, H.K., "The utilization of chiral ion mobility spectrometry for the detection of enantiomeric mixtures and thermally labile compounds" (2013). FIU Electronic Theses and Dissertations, Paper 988. 
[122] Technologies, A. Agilent 6500 Series Q-TOF Techniques and Operation for Small Molecule Applications Course Number R1904A Volume I Student Manual. Agilent Technologies: 2011.

[123] Choi, S.-S., Song, M.J. (2008). Influence of Fragmentor Voltage and Solvent on Negative Ionization Behaviors of Uvinul 3039 Using LC/APCI-MS. Bulletin of the Korean Chemical Society, 29 1847-1849.

[124] Marshall, A.G., Hendrickson, C.L., Shi, S.D.H. (2002). Peer Reviewed: Scaling MS Plateaus with High-Resolution FT-ICRMS. Analytical chemistry, 74 (9): 252 A-259 A.

[125] Fornal, E. (2013). Identification of substituted cathinones: 3,4-Methylenedioxy derivatives by high performance liquid chromatography-quadrupole time of flight mass spectrometry. Journal of Pharmaceutical and Biomedical Analysis, 81-82 (0): 13-19.

[126] Borth, S., Hänsel, W., Rösner, P., Junge, T. (2000). Synthesis of 2,3- and 3,4methylenedioxyphenylalkylamines and their regioisomeric differentiation by mass spectral analysis using GC-MS-MS. Forensic Science International, 114 (3): 139-153.

[127] Kusano, M., Zaitsu, K., Nakayama, H., Nakajima, J., Hisatsune, K., Moriyasu, T., Matsuta, S., Katagi, M., Tsuchihashi, H., Ishii, A. (2015). Positional isomer differentiation of synthetic cannabinoid JWH-081 by GC-MS/MS. Journal of Mass Spectrometry, 50 (3): 586-591.

[128] Gambelunghe, C., Rossi, R., Ferranti, C., Rossi, R., Bacci, M. (2005). Hair analysis by GC/MS/MS to verify abuse of drugs. Journal of Applied Toxicology, 25 (3): 205-211.

[129] Giarrocco, V., Quimby, B., Klee, M. (1997). Retention Time Locking: Concepts and Applications. Agilent Technologies, publication.

[130] Etxebarria, N., Zuloaga, O., Olivares, M., Bartolomé, L.J., Navarro, P. (2009). Retention-time locked methods in gas chromatography. Journal of Chromatography A, 1216 (10): 1624-1629.

[131] Blumberg, L.M., Klee, M.S. (1998). Method translation and retention time locking in partition GC. Analytical chemistry, 70 (18): 3828-3839. 
[132] U.S. Department of Justice Drug Enforcement Administration (2015). List of Controlled Substances. Available at: http://www.deadiversion.usdoj.gov/schedules/index.html [February 2 2015].

[133] Gwak, S., Arroyo-Mora, L.E., Almirall, J.R. (2015). Qualitative analysis of seized synthetic cannabinoids and synthetic cathinones by gas chromatography triple quadrupole tandem mass spectrometry. Drug Testing and Analysis, 7 (2): 121-130.

[134] Uchiyama, N., Kawamura, M., Kikura-Hanajiri, R., Goda, Y. (2011). Identification and quantitation of two cannabimimetic phenylacetylindoles JWH-251 and JWH-250, and four cannabimimetic naphthoylindoles JWH-081, JWH-015, JWH-200, and JWH073 as designer drugs in illegal products. Forensic Toxicology, 29 (1): 25-37.

[135] Uchiyama, N., Kawamura, M., Kikura-Hanajiri, R., Goda, Y. (2012). Identification of two new-type synthetic cannabinoids, N-(1-adamantyl)-1-pentyl-1H-indole-3carboxamide (APICA) and N-(1-adamantyl)-1-pentyl-1H-indazole-3-carboxamide (APINACA), and detection of five synthetic cannabinoids, AM-1220, AM-2233, AM1241, CB-13 (CRA-13), and AM-1248, as designer drugs in illegal products. Forensic Toxicology, 30 (2): 114-125.

[136] Zhang, F., Wang, H., Zhang, L., Zhang, J., Fan, R., Yu, C., Wang, W., Guo, Y. (2014). Suspected-target pesticide screening using gas chromatography-quadrupole timeof-flight mass spectrometry with high resolution deconvolution and retention index/mass spectrum library. Talanta, 128 156-163.

[137] Zhang, F., Yu, C., Wang, W., Fan, R., Zhang, Z., Guo, Y. (2012). Rapid simultaneous screening and identification of multiple pesticide residues in vegetables. Analytica Chimica Acta, 757 39-47.

[138] Portolés, T., Mol, J.G., Sancho, J.V., Hernández, F. (2014). Use of electron ionization and atmospheric pressure chemical ionization in gas chromatography coupled to time-of-flight mass spectrometry for screening and identification of organic pollutants in waters. Journal of Chromatography A, 1339 145-153.

[139] Casado, J., Rodríguez, I., Carpinteiro, I., Ramil, M., Cela, R. (2013). Gas chromatography quadrupole time-of-flight mass spectrometry determination of benzotriazole ultraviolet stabilizers in sludge samples. Journal of Chromatography A, 1293 126-132. 
[140] Agilent Technologies. The New Agilent 7200 Q-TOF for GC/MS. https://www.chem.agilent.com/Library/slidepresentation/Public/Ben_Baars_QTOF.pdf (accessed April 29 2015).

[141] King, L., Kicman, A. (2011). A brief history of 'new psychoactive substances'. Drug Testing and Analysis, 3 (7-8): 401-403.

[142] Holness, H., Jamal, A., Mebel, A., Almirall, J. (2012). Separation mechanism of chiral impurities, ephedrine and pseudoephedrine, found in amphetamine-type substances using achiral modifiers in the gas phase. Analytical and Bioanalytical Chemistry, 404 (8): 2407-2416.

[143] Hilton, C.K., Krueger, C.A., Midey, A.J., Osgood, M., Wu, J., Wu, C. (2010). Improved analysis of explosives samples with electrospray ionization-high resolution ion mobility spectrometry (ESI-HRIMS). International Journal of Mass Spectrometry, 298 (1): 64-71.

[144] Matz, L.M., Hill Jr, H.H., Beegle, L.W., Kanik, I. (2002). Investigation of drift gas selectivity in high resolution ion mobility spectrometry with mass spectrometry detection. Journal of the American Society for Mass Spectrometry, 13 (4): 300-307.

[145] Excellims. RA4100 HPIMS-MS.

http://www.excellims.com/public/downloads/RA4100-Product-Information.pdf (accessed April 29 2015).

[146] Leito, I., Herodes, K., Huopolainen, M., Virro, K., Künnapas, A., Kruve, A., Tanner, R. (2008). Towards the electrospray ionization mass spectrometry ionization efficiency scale of organic compounds. Rapid Communications in Mass Spectrometry, 22 (3): 379-384.

[147] Midey, A.J., Patel, A., Moraff, C., Krueger, C.A., Wu, C. (2013). Improved detection of drugs of abuse using high-performance ion mobility spectrometry with electrospray ionization (ESI-HPIMS) for urine matrices. Talanta, 116 (0): 77-83.

[148] EMS WORLD. Flakka: Rampant designer drug dubbed '\$5 Insanity'. http://www.emsworld.com/news/11904483/flakka-rampant-designer-drug-dubbed-5insanity (accessed April 10 2015). 
[149] National Institute on Drug Abuse. Emerging Trends. http://www.drugabuse.gov/drugs-abuse/emerging-trends (accessed April 19 2015).

[150] Marusich, J.A., Antonazzo, K.R., Wiley, J.L., Blough, B.E., Partilla, J.S., Baumann, M.H. (2014). Pharmacology of novel synthetic stimulants structurally related to the "bath salts" constituent 3,4-methylenedioxypyrovalerone (MDPV).

Neuropharmacology, 87 (0): 206-213.

[151] Matsuta, S., Shima, N., Kamata, H., Kakehashi, H., Nakano, S., Sasaki, K., Kamata, T., Nishioka, H., Miki, A., Katagi, M., Zaitsu, K., Sato, T., Tsuchihashi, H., Suzuki, K. (2015). Metabolism of the designer drug $\alpha$-pyrrolidinobutiophenone ( $\alpha$-PBP) in humans: Identification and quantification of the phase I metabolites in urine. Forensic Science International, 249 (0): 181-188.

[152] Statutes, F. Florida Comprehensive Drug Abuse Prevention and Control Act. In 893.01, Florida Legislature: 2014.

[153] Casale, J.F., Hays, P.A. (2012). The characterization of $\alpha-$ pyrrolidinopentiophenone. Microgram Journal, 9 33-38.

[154] Naylor, J.E., Freeman, K.B., Blough, B.E., Woolverton, W.L., Huskinson, S.L. (2015). Discriminative-stimulus effects of second generation synthetic cathinones in methamphetamine-trained rats. Drug and Alcohol Dependence, 149 (0): 280-284. 


\section{APPENDICES}

Appendix 1. List of compounds investigated in this dissertation and its abbreviations.

\begin{tabular}{|c|c|c|}
\hline & NPS & Abbreviation \\
\hline 1 & (-)-CP 47,497 & \\
\hline 2 & $(-)-$ CP 55,940 & \\
\hline 3 & $(+)-\mathrm{CP} 47,497$ & \\
\hline 4 & (+)-CP 55,940 & \\
\hline 5 & (+)WIN 55212-2 & \\
\hline 6 & ( \pm )3epi CP 47,497-C8-homolog & \\
\hline 7 & $( \pm) 5$-epi CP 55,940 & \\
\hline 8 & $( \pm) \mathrm{CP} 47,497$ & \\
\hline 9 & ( \pm )-CP 47,497-C8-homolog & \\
\hline 10 & $( \pm)-\mathrm{CP} 55,940$ & \\
\hline 11 & ( \pm )-epi CP 47, 497 & \\
\hline 12 & ( \pm WIN 55212 (mesylate) & \\
\hline 13 & (S)-2-Diphenylmethylpyrrolidine & (S)-Desoxy-D2PM \\
\hline 14 & 1-(4-Fluorobenzyl) piperazine & 4-FBP \\
\hline 15 & 1-(p-Fluorophenyl) piperazine & $p$ FPP \\
\hline 16 & 1,4-Dibenzylpiperazine & DBZP \\
\hline 17 & 1'-Naphthoyl Indole & \\
\hline 18 & 2,3-Methylenedioxy Pyrovalerone & 2,3-MDPV \\
\hline 19 & 2,3-Methylenedioxymethcathinone & 2,3-MDMC \\
\hline 20 & 2,3-Pentylone isomer & \\
\hline 21 & 4-iodo-2,5-dimethoxy-N-[(2-methoxyphenyl)methyl]-benzeneethanamine & 25I-NBOMe \\
\hline 22 & 2-Aminoindane & 2-AI \\
\hline 23 & 2,5-Dimethoxy-4-chlorophenethylamine & $2 \mathrm{C}-\mathrm{C}$ \\
\hline 24 & 2,5-Dimethoxy-4-methylphenethylamine & 2C-D \\
\hline
\end{tabular}




\begin{tabular}{|c|c|c|}
\hline & NPS & Abbreviation \\
\hline 25 & 2,5-Dimethoxy-4-ethylphenethylamine & $2 \mathrm{C}-\mathrm{E}$ \\
\hline 26 & 2,5-Dimethoxyphenethylamine & $2 \mathrm{C}-\mathrm{H}$ \\
\hline 27 & 2,5-Dimethoxy-4-iodophenethylamine & $2 \mathrm{C}-\mathrm{I}$ \\
\hline 28 & 2,5-Dimethoxy-4-nitrophenethylamine & $2 \mathrm{C}-\mathrm{N}$ \\
\hline 29 & 2,5-Dimethoxy-4-propylphenethylamine & $2 \mathrm{C}-\mathrm{P}$ \\
\hline 30 & 2,5-Dimethoxy-4-ethylthiophenethylamine & $2 \mathrm{C}-\mathrm{T}-2$ \\
\hline 31 & 2,5-Dimethoxy-4-isopropylthiophenethylamine & $2 \mathrm{C}-\mathrm{T}-4$ \\
\hline 32 & 2-Ethylethcathinone & 2-EEC \\
\hline 33 & 2-Ethylmethcathinone & 2-EMC \\
\hline 34 & 2-Fluoroamphetamine & 2-FA \\
\hline 35 & 2-Fluoroethcathinone & 2-FEC \\
\hline 36 & 2-Fluoromethamphetamine & 2-FMA \\
\hline 37 & 2-Fluoromethcathinone & 2-FMC \\
\hline 38 & 2-Methoxymethcathinone & 2-MeOMC \\
\hline 39 & 2-Methylethcathinone & 2-MEC \\
\hline 40 & 2-Methylmethcathinone & 2-MMC \\
\hline 41 & 2-Methyl- $\alpha$-pyrrolidinobutiophenone & 2-MePBP \\
\hline 42 & 3,4-Dimethoxymethamphetamine & 3,4-DMMA \\
\hline 43 & 3,4-Dimethylmethcathinone & 3,4-DMMC \\
\hline 44 & 3',4'-Methylenedioxy- $\alpha$-pyrrolidinobutiophenone & 3',4'-MDPBP \\
\hline 45 & 3',4'-Methylenedioxy- $\alpha$-pyrrolidinopropiophenone & 3',4'-MDPPP \\
\hline 46 & 3-Ethylethcathinone & 3-EEC \\
\hline 47 & 3-Ethylmethcathinone & 3-EMC \\
\hline 48 & 3-Fluoroamphetamine & 3-FA \\
\hline 49 & 3-Fluoroethcathinone & 3-FEC \\
\hline 50 & 3-Fluoromethamphetamine & 3-FMA \\
\hline
\end{tabular}




\begin{tabular}{|c|c|c|}
\hline & NPS & Abbreviation \\
\hline 51 & 3-Fluoromethcathinone & 3-FMC \\
\hline 52 & 3-Methoxymethcathinone & 3-MeOMC \\
\hline 53 & 3-Methylethcathinone & 3-MEC \\
\hline 54 & 3-Methylmethcathinone & 3-MMC \\
\hline 55 & 3-Methyl- $\alpha$-pyrrolidinobutiophenone & 3-МеРBP \\
\hline 56 & 4-Ethylethcathinone & 4-EEC \\
\hline 57 & 4-Ethylmethcathinone & 4-EMC \\
\hline 58 & 4-ethyl-N,N-Dimethylcathinone & 4-ethyl-N,N-DMC \\
\hline 59 & 4-Fluoroamphetamine & 4-FA \\
\hline 60 & 4-Fluoroethcathinone & 4-FEC \\
\hline 61 & 4-Fluoromethamphetamine & 4-FMA \\
\hline 62 & 4-Fluoromethcathinone & 4-FMC \\
\hline 63 & 4-Methylethcathinone & 4-MEC \\
\hline 64 & 4-methoxy-N,N-Dimethyltryptamine & 4-MeO DMT \\
\hline 65 & 4'-Methoxy- $\alpha$-pyrrolidinopropiophenone (tosylate) & 4'-MeOPPP \\
\hline 66 & 4-Methyl- $\alpha$-pyrrolidinobutiophenone & 4-MePBP \\
\hline 67 & 4'-Methyl- $\alpha$-pyrrolidinohexanophenone & 4'-MePHP \\
\hline 68 & 4'-Methyl- $\alpha$-pyrrolidinopropiophenone & 4'-MePPP \\
\hline 69 & 4-Methylmethcathinone & 4-MMC \\
\hline 70 & 4-Quinolone-3-Carboxamide CB2 Ligand & 4Q3C CB2 Ligand \\
\hline 71 & 5-(2-Aminopropyl) Benzofuran & $5-\mathrm{APB}$ \\
\hline 72 & 5-iodo-2-Aminoindane & 5-IAI \\
\hline 73 & 5-methoxy-N,N-Dimethyltryptamine & 5-MeO-DMT \\
\hline 74 & N,N-Diallyl-5-Methoxytryptamine & $\begin{array}{c}\text { 5-methoxy } \\
\text { DALT }\end{array}$ \\
\hline 75 & 5-methoxy-N-methyl-N-(1-methylethyl)-1H-Indole-3-ethanamine & 5-MeO MiPT \\
\hline
\end{tabular}




\begin{tabular}{|c|c|c|}
\hline & NPS & Abbreviation \\
\hline 76 & 9-Octadecenamide & Oleamide \\
\hline 77 & APINACA & AKB48 \\
\hline 78 & AM1220 & \\
\hline 79 & AM1235 & \\
\hline 80 & AM1248 & \\
\hline 81 & AM2201 & \\
\hline 82 & AM2201 2'-naphthyl isomer & \\
\hline 83 & AM2201 4-methylnaphthyl analog & MAM2201 \\
\hline 84 & AM2201 N-(2-fluoropentyl) isomer & \\
\hline 85 & AM2201 N-(3-fluoropentyl) isomer & \\
\hline 86 & AM2201 N-(4-fluoropentyl) isomer & \\
\hline 87 & AM2232 & \\
\hline 88 & AM2233 & \\
\hline 89 & AM679 & \\
\hline 90 & AM694 & \\
\hline 91 & AM694 3-iodo isomer & \\
\hline 92 & AM694 4-iodo isomer & \\
\hline 93 & Dibutylone & bk-DMBDB \\
\hline 94 & Dimethylone & bk-MDDMA \\
\hline 95 & bk-Methylenedioxyethylamphetamine (Ethylone) & bk-MDEA \\
\hline 96 & $\alpha$-methylamino-Butyrophenone & Buphedrone \\
\hline 97 & $\beta$-keto MBDB & Butylone \\
\hline 98 & Cannabidiol & CBD \\
\hline 99 & Cannabipiperidiethanone & $\mathrm{CPE}$ \\
\hline 100 & 1-naphthalenyl[4-(pentylox)-1-naphthalenyl]-methanone (CRA-13) & CB-13 \\
\hline 101 & Diphenyl-2-pyrrolidinemethanol & $\mathrm{D} 2 \mathrm{PM}$ \\
\hline
\end{tabular}




\begin{tabular}{|c|c|c|}
\hline & NPS & Abbreviation \\
\hline 102 & 2-Diphenylmethylpiperidine (Desoxypipradrol) & 2-DPMP \\
\hline 103 & Diethylcathinone & DEC \\
\hline 104 & N-Ethylcathinone & Ethcathinone \\
\hline 105 & $\beta$-keto-Ethylbenzodioxolylbutanamine (Eutylone) & bk-EBDB \\
\hline 106 & Harmaline & \\
\hline 107 & HU-210 & \\
\hline 108 & HU-211 & \\
\hline 109 & HU-308 & \\
\hline 110 & Indomethacin Morpholinylamide & IMMA \\
\hline 111 & JWH 007 & \\
\hline 112 & JWH 011 & \\
\hline 113 & JWH 015 & \\
\hline 114 & JWH 016 & \\
\hline 115 & JWH 018 & \\
\hline 116 & JWH 018 2'-naphthyl-N-(1-ethylpropyl) & \\
\hline 117 & JWH 018 2'-naphthyl isomer & \\
\hline 118 & JWH 018 2'naphthyl-N-(1,1-dimethylpropyl) isomer & \\
\hline 119 & JWH 018 2'-naphthyl-N-(1,2-dimethylpropyl) isomer & \\
\hline 120 & JWH 018 2'-naphthyl-N-(1-methylbutyl) isomer & \\
\hline 121 & JWH 018 2'-naphthyl-N-(2,2-dimethylpropyl) isomer & \\
\hline 122 & JWH 018 2'-naphthyl-N-(2-methylbutyl) isomer & \\
\hline 123 & JWH 018 2'-naphthyl-N-(3-methylbutyl) isomer & \\
\hline 124 & JWH 018 6-methoxyindole analog & \\
\hline 125 & JWH 018 adamantyl analog & \\
\hline 126 & JWH 018 adamantyl carboxamide & \\
\hline 127 & JWH $018 \mathrm{~N}$-(1,1-dimethylpropyl) isomer & \\
\hline
\end{tabular}




\begin{tabular}{|c|c|c|}
\hline & NPS & Abbreviation \\
\hline 128 & JWH 018 N-(1,2-dimethylpropyl) isomer & \\
\hline 129 & JWH 018 N-(1-ethylpropyl) isomer & \\
\hline 130 & JWH 018 N-(1-methylbutyl) isomer & \\
\hline 131 & JWH $018 \mathrm{~N}$-(2,2dimethylpropyl) isomer & \\
\hline 132 & JWH 018 N-(2-methylbutyl) isomer & \\
\hline 133 & JWH $018 \mathrm{~N}$-(3-methylbutyl) isomer & \\
\hline 134 & JWH 018 N-(4,5-epoxypentyl) analog & \\
\hline 135 & JWH 018 N-(5-bromopentyl) analog & \\
\hline 136 & JWH $018 \mathrm{~N}$-(5-chloropentyl) analog & \\
\hline 137 & JWH 019 & \\
\hline 138 & JWH 020 & \\
\hline 139 & JWH 022 & \\
\hline 140 & JWH 030 & \\
\hline 141 & JWH 031 & \\
\hline 142 & JWH 072 & \\
\hline 143 & JWH 073 & \\
\hline 144 & JWH 073 2-methylnaphthyl analog & \\
\hline 145 & JWH 073 2'-naphthyl isomer & \\
\hline 146 & JWH 073 2'-naphthyl-N-(1,1-dimethylethyl) isomer & \\
\hline 147 & JWH 073 2'-naphthyl-N-(1-methylpropyl) isomer & \\
\hline 148 & JWH 073 2'-naphthyl-N-(2-methylpropyl) isomer & \\
\hline 149 & JWH 073 4-methylnaphthyl analog & \\
\hline 150 & JWH 073 N-(1,1-dimethylethyl) isomer & \\
\hline 151 & JWH $073 \mathrm{~N}$-(1-methylpropyl) isomer & \\
\hline 152 & JWH 073 N-(2-methylpropyl) isomer & \\
\hline 153 & JWH 081 & \\
\hline
\end{tabular}




\begin{tabular}{|c|c|c|}
\hline & NPS & Abbreviation \\
\hline 154 & JWH 081 2-methoxynaphthyl isomer & \\
\hline 155 & JWH 081 3-methoxynaphthyl isomer & \\
\hline 156 & JWH 081 5-methoxynaphthyl isomer & \\
\hline 157 & JWH 081 6-methoxynaphthyl isomer & \\
\hline 158 & JWH 081 7-methoxynaphthyl isomer & \\
\hline 159 & JWH 081 8-methoxynaphthyl isomer & \\
\hline 160 & JWH 098 & \\
\hline 161 & JWH 122 & \\
\hline 162 & JWH 122 2-methylnaphthyl isomer & \\
\hline 163 & JWH 122 3-methylnaphthyl isomer & \\
\hline 164 & JWH 122 5-methylnaphthyl isomer & \\
\hline 165 & JWH 122 6-methylnaphthyl isomer & \\
\hline 166 & JWH 122 7-methylnaphthyl isomer & \\
\hline 167 & JWH 122 8-methylnaphthyl isomer & \\
\hline 168 & JWH 122 N-(4-pentenyl) Analog & \\
\hline 169 & JWH 145 & \\
\hline 170 & JWH 147 & \\
\hline 171 & JWH 175 & \\
\hline 172 & JWH 180 & \\
\hline 173 & JWH 182 & \\
\hline 174 & JWH 200 & \\
\hline 175 & JWH 200 2'-naphthyl isomer & \\
\hline 176 & JWH 201 & \\
\hline 177 & JWH 203 & \\
\hline 178 & JWH 203 3-chlorophenyl isomer & \\
\hline 179 & JWH 203 4-chlorophenyl isomer & \\
\hline
\end{tabular}




\begin{tabular}{|c|c|c|}
\hline & NPS & Abbreviation \\
\hline 180 & JWH 210 & \\
\hline 181 & JWH 210 2-ethylnaphthyl isomer & \\
\hline 182 & JWH 210 3-ethylnaphthyl isomer & \\
\hline 183 & JWH 210 5-ethylnaphthyl isomer & \\
\hline 184 & JWH 210 6-ethylnaphthyl isomer & \\
\hline 185 & JWH 210 7-ethylnaphthyl isomer & \\
\hline 186 & JWH 210 8-ethylnaphthyl isomer & \\
\hline 187 & JWH 249 & \\
\hline 188 & JWH 250 & \\
\hline 189 & JWH 251 & \\
\hline 190 & JWH 251 3-methylphenyl isomer & \\
\hline 191 & JWH 251 4-methylphenyl isomer & \\
\hline 192 & JWH 302 & \\
\hline 193 & JWH 307 & \\
\hline 194 & JWH 309 & \\
\hline 195 & JWH 368 & \\
\hline 196 & JWH 369 & \\
\hline 197 & JWH 370 & \\
\hline 198 & JWH 398 & \\
\hline 199 & JWH 398 2-chloronaphthyl isomer & \\
\hline 200 & JWH 398 3-chloronaphthyl isomer & \\
\hline 201 & JWH 398 5-chloronaphthyl isomer & \\
\hline 202 & JWH 398 6-chloronaphthyl isomer & \\
\hline 203 & JWH 398 7-chloronaphthyl isomer & \\
\hline 204 & JWH 398 8-chloronaphthyl isomer & \\
\hline 205 & JWH 424 & \\
\hline
\end{tabular}




\begin{tabular}{|c|c|c|}
\hline & NPS & Abbreviation \\
\hline 206 & (2Z)-2-(1-hexyl-1,2-dihydro-2-oxo-3H-indol-3-ylidene)hydrazide, benzoic acid & MDA 19 \\
\hline 207 & 5,6-Methylenedioxy-2-aminoindane & MDAI \\
\hline 208 & MDMA methylene homolog & \\
\hline 209 & 3,4-Methylenedioxy Pyrovalerone & MDPV \\
\hline 210 & Meconin & \\
\hline 211 & para-Methoxymethcathinone & Methedrone \\
\hline 212 & Methoxetamine & MXE \\
\hline 213 & 3,4-Methylenedioxy-N-methylcathinone (bk-MDMA) & Methylone \\
\hline 214 & 9-methoxy Corynantheidine (Kratom) & Mitragynine \\
\hline 215 & N,N-Dimethylcathinone & $\mathrm{N}, \mathrm{N}-\mathrm{DMC}$ \\
\hline 216 & Naphpyrovalerone & Naphyrone \\
\hline 217 & Naphyrone 1-naphthyl isomer & $\alpha$ - Naphyrone \\
\hline 218 & Nor-Mephedrone & \\
\hline 219 & Norsufentanil & \\
\hline 220 & $\alpha$-methylamino-Valerophenone & Pentedrone \\
\hline 221 & Pentylone & \\
\hline 222 & Phenazepam & \\
\hline 223 & 1-phenyl-piperazine & Phenylpiperazine \\
\hline 224 & WIN 48,098 & Pravadoline \\
\hline 225 & Pyrovalerone & \\
\hline 226 & RCS-4 & \\
\hline 227 & RCS-4 2-methoxy isomer & \\
\hline 228 & RCS-4 3-methoxy isomer & \\
\hline 229 & RCS-4-C4 Homolog & \\
\hline 230 & RCS-8 & \\
\hline 231 & RCS-8 3-methoxy isomer & \\
\hline
\end{tabular}




\begin{tabular}{|c|c|c|}
\hline & NPS & Abbreviation \\
\hline 232 & RCS-8 4-methoxy isomer & \\
\hline 233 & $\mathrm{~N}$-adamantyl-1-fluoropentylindole-3-Carboxamide (5F-APICA) & STS-135 \\
\hline 234 & 1-(m-Trifluoromethylphenyl) piperazine (hydrochloride) & TFMPP \\
\hline 235 & 2,4,5-Trimethoxyamphetamine & TMA-2 \\
\hline 236 & UR-144 & \\
\hline 237 & (3'-(aminocarbonyl)[1,1'-biphenyl]-3-yl)-cyclohexylcarbamate & URB597 \\
\hline 238 & [1,1'-biphenyl]-3-yl-carbamic acid, cyclohexyl ester & URB602 \\
\hline 239 & 6-methyl-2-[(4-methylphenyl)amino]-1-benzoxazin-4-one & URB754 \\
\hline 240 & 6-Bromopravadoline & WIN 54,461 \\
\hline 241 & 5-fluoro UR-144 & XLR-11 \\
\hline 242 & $\alpha$-pyrrolidinobutiophenone & $\alpha-\mathrm{PBP}$ \\
\hline 243 & $\alpha$-pyrrolidinopentiophenone & $\alpha-P P P$ \\
\hline 244 & $\alpha$-pyrrolidinopropiophenone & $\alpha-P V P$ \\
\hline
\end{tabular}

Appendix 2. Fragmentation pattern of each substance at the collision energy of $20 \mathrm{eV}$. Theoretical mass was calculated using a mass calculator tool from Agilent MassHunter Qualitative Analysis.

\begin{tabular}{|c|c|c|c|c|c|c|c|}
\hline NPS & $\begin{array}{l}\text { Molecular } \\
\text { formula }\end{array}$ & $\begin{array}{c}\text { Theoretical } \\
\text { mass }(m / z)\end{array}$ & $\begin{array}{r}\text { Measured } \\
\text { mass }(m / z)\end{array}$ & $\begin{array}{c}\text { Mass } \\
\text { difference } \\
(\mathrm{m} / \mathrm{z})\end{array}$ & $\begin{array}{c}\text { Relative } \\
\text { abundance } \\
(\%)\end{array}$ & $\begin{array}{c}\text { Measured } \\
\text { mass loss } \\
(\mathrm{m} / \mathrm{z})\end{array}$ & $\begin{array}{c}\text { Fragment } \\
\text { formula }\end{array}$ \\
\hline \multicolumn{8}{|c|}{ Synthetic cathinones } \\
\hline \multirow{6}{*}{ 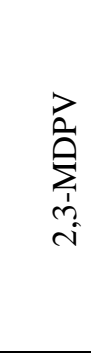 } & $\mathrm{C}_{16} \mathrm{H}_{22} \mathrm{NO}_{3}$ & 276.1594 & 276.1588 & 0.0006 & 99.15 & & \\
\hline & $\mathrm{C}_{11} \mathrm{H}_{11} \mathrm{NO}_{3}$ & 205.0733 & 205.0856 & -0.0123 & 11.55 & 71.0732 & $\mathrm{C}_{5} \mathrm{H}_{11}$ \\
\hline & $\mathrm{C}_{11} \mathrm{H}_{11} \mathrm{O}_{2}$ & 175.0754 & 175.0750 & 0.0004 & 76.69 & 101.0838 & $\mathrm{C}_{5} \mathrm{H}_{11} \mathrm{NO}$ \\
\hline & $\mathrm{C}_{8} \mathrm{H}_{7} \mathrm{O}_{2}$ & 135.0441 & 135.0442 & -0.0001 & 100.00 & 141.1146 & $\mathrm{C}_{8} \mathrm{H}_{15} \mathrm{NO}$ \\
\hline & $\mathrm{C}_{8} \mathrm{H}_{16} \mathrm{~N}$ & 126.1277 & 126.1271 & 0.0006 & 33.16 & 150.0317 & $\mathrm{C}_{8} \mathrm{H}_{6} \mathrm{O}_{3}$ \\
\hline & $\mathrm{C}_{4} \mathrm{H}_{8} \mathrm{~N}$ & 70.0651 & 70.0663 & -0.0012 & 7.85 & 206.0925 & $\mathrm{C}_{12} \mathrm{H}_{14} \mathrm{O}_{3}$ \\
\hline 0 & $\mathrm{C}_{16} \mathrm{H}_{22} \mathrm{NO}_{3}$ & 276.1594 & 276.1592 & 0.0002 & 100.00 & & \\
\hline
\end{tabular}




\begin{tabular}{|c|c|c|c|c|c|c|c|}
\hline NPS & $\begin{array}{l}\text { Molecular } \\
\text { formula }\end{array}$ & $\begin{array}{l}\text { Theoretical } \\
\text { mass }(m / z)\end{array}$ & $\begin{array}{r}\text { Measured } \\
\text { mass }(m / z)\end{array}$ & $\begin{array}{c}\text { Mass } \\
\text { difference } \\
(\mathrm{m} / \mathrm{z})\end{array}$ & $\begin{array}{l}\text { Relative } \\
\text { abundance } \\
(\%)\end{array}$ & $\begin{array}{l}\text { Measured } \\
\text { mass loss } \\
(\mathrm{m} / \mathrm{z})\end{array}$ & $\begin{array}{c}\text { Fragment } \\
\text { formula }\end{array}$ \\
\hline & $\mathrm{C}_{11} \mathrm{H}_{11} \mathrm{NO}_{3}$ & 205.0733 & 205.0863 & -0.0130 & 65.56 & 71.0729 & $\mathrm{C}_{4} \mathrm{H}_{9}$ \\
\hline & $\mathrm{C}_{11} \mathrm{H}_{11} \mathrm{O}_{2}$ & 175.0754 & 175.0751 & 0.0003 & 63.85 & 101.0841 & $\mathrm{C}_{5} \mathrm{H}_{11} \mathrm{NO}$ \\
\hline & $\mathrm{C}_{8} \mathrm{H}_{5} \mathrm{O}_{3}$ & 149.0233 & 149.0328 & -0.0095 & 36.19 & 127.1264 & $\mathrm{C}_{8} \mathrm{H}_{17} \mathrm{~N}$ \\
\hline & $\mathrm{C}_{8} \mathrm{H}_{7} \mathrm{O}_{2}$ & 135.0441 & 135.0440 & 0.0001 & 58.84 & 141.1152 & $\mathrm{C}_{8} \mathrm{H}_{15} \mathrm{NO}$ \\
\hline & $\mathrm{C}_{8} \mathrm{H}_{16} \mathrm{~N}$ & 126.1277 & 126.1273 & 0.0004 & 67.12 & 150.0319 & $\mathrm{C}_{8} \mathrm{H}_{6} \mathrm{O}_{3}$ \\
\hline & $\mathrm{C}_{5} \mathrm{H}_{10} \mathrm{~N}$ & 84.0808 & 84.0813 & -0.0005 & 9.05 & 192.0779 & $\mathrm{C}_{11} \mathrm{H}_{12} \mathrm{O}_{3}$ \\
\hline \multirow{7}{*}{ 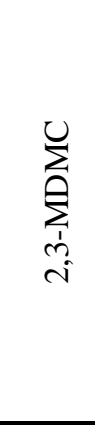 } & $\mathrm{C}_{11} \mathrm{H}_{14} \mathrm{NO}_{3}$ & 208.0968 & 208.0961 & 0.0007 & 0.57 & & \\
\hline & $\mathrm{C}_{11} \mathrm{H}_{12} \mathrm{NO}_{2}$ & 190.0863 & 190.0887 & -0.0024 & 3.79 & 18.0074 & $\mathrm{H}_{2} \mathrm{O}$ \\
\hline & $\mathrm{C}_{10} \mathrm{H}_{8} \mathrm{O}_{2}$ & 160.0519 & 160.0765 & -0.0246 & 100.00 & 48.0196 & $\mathrm{CH}_{6} \mathrm{NO}$ \\
\hline & $\mathrm{C}_{9} \mathrm{H}_{7} \mathrm{O}_{2}$ & 147.0441 & 147.0446 & -0.0005 & 10.94 & 61.0515 & $\mathrm{C}_{2} \mathrm{H}_{7} \mathrm{NO}$ \\
\hline & $\mathrm{C}_{9} \mathrm{H}_{10} \mathrm{~N}$ & 132.0808 & 132.0809 & -0.0001 & 42.53 & 76.0152 & $\mathrm{C}_{2} \mathrm{H}_{4} \mathrm{O}_{3}$ \\
\hline & $\mathrm{C}_{8} \mathrm{H}_{7} \mathrm{~N}$ & 117.0573 & 117.0580 & -0.0007 & 3.05 & 91.0381 & $\mathrm{C}_{3} \mathrm{H}_{7} \mathrm{O}_{3}$ \\
\hline & $\mathrm{C}_{7} \mathrm{H}_{7}$ & 91.0542 & 91.0543 & -0.0001 & 4.23 & 117.0418 & $\mathrm{C}_{4} \mathrm{H}_{7} \mathrm{NO}_{3}$ \\
\hline \multirow{7}{*}{ 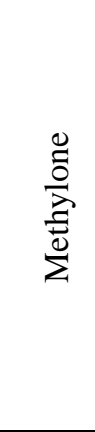 } & $\mathrm{C}_{11} \mathrm{H}_{14} \mathrm{NO}_{3}$ & 208.0968 & 208.0949 & 0.0019 & 0.82 & & \\
\hline & $\mathrm{C}_{11} \mathrm{H}_{12} \mathrm{NO}_{2}$ & 190.0863 & 190.0877 & -0.0014 & 13.32 & 18.0072 & $\mathrm{H}_{2} \mathrm{O}$ \\
\hline & $\mathrm{C}_{10} \mathrm{H}_{8} \mathrm{O}_{2}$ & 160.0519 & 160.0768 & -0.0249 & 100.00 & 48.0181 & $\mathrm{CH}_{6} \mathrm{NO}$ \\
\hline & $\mathrm{C}_{9} \mathrm{H}_{7} \mathrm{O}_{2}$ & 147.0441 & 147.0452 & -0.0011 & 7.64 & 61.0497 & $\mathrm{C}_{2} \mathrm{H}_{7} \mathrm{NO}$ \\
\hline & $\mathrm{C}_{9} \mathrm{H}_{10} \mathrm{~N}$ & 132.0808 & 132.0816 & -0.0008 & 32.80 & 76.0133 & $\mathrm{C}_{2} \mathrm{H}_{4} \mathrm{O}_{3}$ \\
\hline & $\mathrm{C}_{8} \mathrm{H}_{7} \mathrm{~N}$ & 117.0573 & 117.0581 & -0.0005 & 1.71 & 91.0371 & $\mathrm{C}_{3} \mathrm{H}_{7} \mathrm{O}_{3}$ \\
\hline & $\mathrm{C}_{7} \mathrm{H}_{7}$ & 91.0542 & 91.0530 & 0.0012 & 2.05 & 117.0419 & $\mathrm{C}_{4} \mathrm{H}_{7} \mathrm{NO}_{3}$ \\
\hline \multirow{7}{*}{ 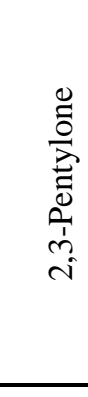 } & $\mathrm{C}_{13} \mathrm{H}_{18} \mathrm{NO}_{3}$ & 236.1281 & 236.1246 & 0.0035 & 1.39 & & \\
\hline & $\mathrm{C}_{13} \mathrm{H}_{16} \mathrm{NO}_{2}$ & 218.1176 & 218.1176 & 0.0000 & 18.84 & 17.8824 & $\mathrm{H}_{2} \mathrm{O}$ \\
\hline & $\mathrm{C}_{12} \mathrm{H}_{12} \mathrm{O}_{2}$ & 188.0832 & 188.1068 & -0.0236 & 100.00 & 47.8932 & $\mathrm{CH}_{4} \mathrm{NO}$ \\
\hline & $\mathrm{C}_{11} \mathrm{H}_{11} \mathrm{O}_{2}$ & 175.0754 & 175.0751 & 0.0003 & 41.08 & 60.9249 & $\mathrm{C}_{2} \mathrm{H}_{7} \mathrm{NO}$ \\
\hline & $\mathrm{C}_{11} \mathrm{H}_{14} \mathrm{~N}$ & 160.1121 & 160.1117 & 0.0004 & 23.59 & 75.8883 & $\mathrm{C}_{2} \mathrm{H}_{4} \mathrm{O}_{3}$ \\
\hline & $\mathrm{C}_{8} \mathrm{H}_{7} \mathrm{O}_{2}$ & 135.0441 & 135.0437 & 0.0004 & 45.90 & 100.9563 & $\mathrm{C}_{5} \mathrm{H}_{11} \mathrm{NO}$ \\
\hline & $\mathrm{C}_{5} \mathrm{H}_{12} \mathrm{~N}$ & 86.0964 & 86.0970 & -0.0006 & 11.82 & 149.9030 & $\mathrm{C}_{8} \mathrm{H}_{6} \mathrm{O}_{3}$ \\
\hline \multirow{5}{*}{ 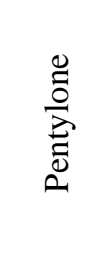 } & $\mathrm{C}_{13} \mathrm{H}_{18} \mathrm{NO}_{3}$ & 236.1281 & 236.1294 & -0.0013 & 1.89 & & \\
\hline & $\mathrm{C}_{13} \mathrm{H}_{16} \mathrm{NO}_{2}$ & 218.1176 & 218.1176 & 0.0000 & 23.76 & 17.8824 & $\mathrm{H}_{2} \mathrm{O}$ \\
\hline & $\mathrm{C}_{12} \mathrm{H}_{12} \mathrm{O}_{2}$ & 188.0832 & 188.1071 & -0.0239 & 100.00 & 47.8929 & $\mathrm{CH}_{4} \mathrm{NO}$ \\
\hline & $\mathrm{C}_{11} \mathrm{H}_{11} \mathrm{O}_{2}$ & 175.0754 & 175.0688 & 0.0066 & 38.13 & 60.9312 & $\mathrm{C}_{2} \mathrm{H}_{7} \mathrm{NO}$ \\
\hline & $\mathrm{C}_{11} \mathrm{H}_{14} \mathrm{~N}$ & 160.1121 & 160.1113 & 0.0008 & 10.64 & 76.0000 & $\mathrm{C}_{2} \mathrm{H}_{4} \mathrm{O}_{3}$ \\
\hline
\end{tabular}




\begin{tabular}{|c|c|c|c|c|c|c|c|}
\hline NPS & $\begin{array}{l}\text { Molecular } \\
\text { formula }\end{array}$ & $\begin{array}{l}\text { Theoretical } \\
\text { mass }(m / z)\end{array}$ & $\begin{array}{r}\text { Measured } \\
\text { mass }(m / z)\end{array}$ & $\begin{array}{c}\text { Mass } \\
\text { difference } \\
(\mathrm{m} / \mathrm{z})\end{array}$ & $\begin{array}{l}\text { Relative } \\
\text { abundance } \\
(\%)\end{array}$ & $\begin{array}{c}\text { Measured } \\
\text { mass loss } \\
(\mathrm{m} / \mathrm{z})\end{array}$ & $\begin{array}{c}\text { Fragment } \\
\text { formula }\end{array}$ \\
\hline & $\mathrm{C}_{8} \mathrm{H}_{7} \mathrm{O}_{2}$ & 135.0441 & 135.0438 & 0.0003 & 19.10 & 100.9562 & $\mathrm{C}_{5} \mathrm{H}_{11} \mathrm{NO}$ \\
\hline & $\mathrm{C}_{5} \mathrm{H}_{12} \mathrm{~N}$ & 86.0964 & 86.0971 & -0.0007 & 24.15 & 149.9029 & $\mathrm{C}_{8} \mathrm{H}_{6} \mathrm{O}_{3}$ \\
\hline \multirow{7}{*}{ 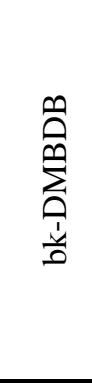 } & $\mathrm{C}_{13} \mathrm{H}_{18} \mathrm{NO}_{3}$ & 236.1281 & 236.1289 & -0.0008 & 19.24 & & \\
\hline & $\mathrm{C}_{11} \mathrm{H}_{13} \mathrm{NO}_{2}$ & 191.0941 & 191.0709 & 0.0232 & 38.91 & 45.0580 & $\mathrm{C}_{2} \mathrm{H}_{5} \mathrm{O}$ \\
\hline & $\mathrm{C}_{10} \mathrm{H}_{9} \mathrm{O}_{2}$ & 161.0597 & 161.0605 & -0.0008 & 74.77 & 75.0684 & $\mathrm{C}_{3} \mathrm{H}_{9} \mathrm{NO}$ \\
\hline & $\mathrm{C}_{8} \mathrm{H}_{5} \mathrm{O}_{3}$ & 149.0233 & 149.0238 & -0.0005 & 52.54 & 87.1051 & $\mathrm{C}_{5} \mathrm{H}_{13} \mathrm{~N}$ \\
\hline & $\mathrm{C}_{10} \mathrm{H}_{13}$ & 133.1012 & 133.0654 & 0.0358 & 17.85 & 103.0635 & $\mathrm{C}_{3} \mathrm{H}_{5} \mathrm{NO}_{3}$ \\
\hline & $\mathrm{C}_{8} \mathrm{H}_{9}$ & 105.0699 & 105.0705 & -0.0006 & 9.32 & 131.0584 & $\mathrm{C}_{5} \mathrm{H}_{9} \mathrm{NO}_{3}$ \\
\hline & $\mathrm{C}_{5} \mathrm{H}_{12} \mathrm{~N}$ & 86.0964 & 86.0972 & -0.0008 & 100.00 & 150.0317 & $\mathrm{C}_{8} \mathrm{H}_{6} \mathrm{O}_{3}$ \\
\hline \multirow{5}{*}{ 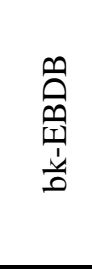 } & $\mathrm{C}_{13} \mathrm{H}_{18} \mathrm{NO}_{3}$ & 236.1281 & 236.1298 & -0.0017 & 2.47 & & \\
\hline & $\mathrm{C}_{13} \mathrm{H}_{16} \mathrm{NO}_{2}$ & 218.1176 & 218.1187 & -0.0011 & 35.15 & 18.0111 & $\mathrm{H}_{2} \mathrm{O}$ \\
\hline & $\mathrm{C}_{12} \mathrm{H}_{12} \mathrm{O}_{2}$ & 188.0832 & 188.1079 & -0.0247 & 100.00 & 48.0219 & $\mathrm{CH}_{5} \mathrm{NO}$ \\
\hline & $\mathrm{C}_{10} \mathrm{H}_{9} \mathrm{O}_{2}$ & 161.0597 & 161.0616 & -0.0019 & 17.22 & 75.0682 & $\mathrm{C}_{3} \mathrm{H}_{9} \mathrm{NO}$ \\
\hline & $\mathrm{C}_{5} \mathrm{H}_{12} \mathrm{~N}$ & 86.0964 & 86.0974 & -0.0010 & 16.15 & 150.0324 & $\mathrm{C}_{8} \mathrm{H}_{6} \mathrm{O}_{3}$ \\
\hline \multirow{8}{*}{ 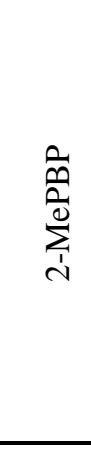 } & $\mathrm{C}_{15} \mathrm{H}_{2 \curlywedge} \mathrm{NO}$ & 232.1696 & 232.1692 & 0.0004 & 92.96 & & \\
\hline & $\mathrm{C}_{13} \mathrm{H}_{17} \mathrm{NO}$ & 203.1305 & 203.1268 & 0.0037 & 3.60 & 29.0424 & $\mathrm{C}_{2} \mathrm{H}_{5}$ \\
\hline & $\mathrm{C}_{11} \mathrm{H}_{13} \mathrm{O}$ & 161.0961 & 161.0943 & 0.0018 & 68.35 & 71.0749 & $\mathrm{C}_{4} \mathrm{H}_{9} \mathrm{~N}$ \\
\hline & $\mathrm{C}_{10} \mathrm{H}_{13}$ & 133.1012 & 133.1009 & 0.0003 & 31.74 & 99.0683 & $\mathrm{C}_{5} \mathrm{H}_{9} \mathrm{NO}$ \\
\hline & $\mathrm{C}_{8} \mathrm{H}_{7} \mathrm{O}$ & 119.0491 & 119.0493 & -0.0002 & 50.18 & 113.1199 & $\mathrm{C}_{7} \mathrm{H}_{15} \mathrm{~N}$ \\
\hline & $\mathrm{C}_{7} \mathrm{H}_{14} \mathrm{~N}$ & 112.1121 & 112.1128 & -0.0007 & 75.09 & 120.0564 & $\mathrm{C}_{8} \mathrm{H}_{8} \mathrm{O}$ \\
\hline & $\mathrm{C}_{8} \mathrm{H}_{9}$ & 105.0699 & 105.0698 & 0.0001 & 100.00 & 127.0994 & $\mathrm{C}_{7} \mathrm{H}_{13} \mathrm{NO}$ \\
\hline & $\mathrm{C}_{4} \mathrm{H}_{8} \mathrm{~N}$ & 70.0651 & 70.0637 & 0.0014 & 14.41 & 162.1055 & $\mathrm{C}_{11} \mathrm{H}_{14} \mathrm{O}$ \\
\hline \multirow{8}{*}{ 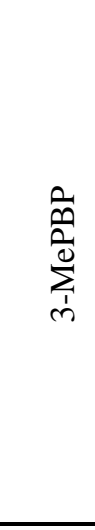 } & $\mathrm{C}_{15} \mathrm{H}_{22} \mathrm{NO}$ & 232.1696 & 232.1699 & -0.0003 & 67.42 & & \\
\hline & $\mathrm{C}_{13} \mathrm{H}_{17} \mathrm{NO}$ & 203.1305 & 203.1301 & 0.0004 & 7.12 & 29.0398 & $\mathrm{C}_{2} \mathrm{H}_{5}$ \\
\hline & $\mathrm{C}_{11} \mathrm{H}_{13} \mathrm{O}$ & 161.0961 & 161.0955 & 0.0006 & 47.99 & 71.0744 & $\mathrm{C}_{4} \mathrm{H}_{9} \mathrm{~N}$ \\
\hline & $\mathrm{C}_{10} \mathrm{H}_{13}$ & 133.1012 & 133.1010 & 0.0002 & 29.12 & 99.0689 & $\mathrm{C}_{5} \mathrm{H}_{9} \mathrm{NO}$ \\
\hline & $\mathrm{C}_{8} \mathrm{H}_{7} \mathrm{O}$ & 119.0491 & 119.0486 & 0.0005 & 22.22 & 113.1213 & $\mathrm{C}_{7} \mathrm{H}_{15} \mathrm{~N}$ \\
\hline & $\mathrm{C}_{7} \mathrm{H}_{14} \mathrm{~N}$ & 112.1121 & 112.1112 & 0.0009 & 31.16 & 120.0587 & $\mathrm{C}_{8} \mathrm{H}_{8} \mathrm{O}$ \\
\hline & $\mathrm{C}_{8} \mathrm{H}_{9}$ & 105.0699 & 105.0699 & 0.0000 & 100.00 & 127.1000 & $\mathrm{C}_{7} \mathrm{H}_{13} \mathrm{NO}$ \\
\hline & $\mathrm{C}_{4} \mathrm{H}_{8} \mathrm{~N}$ & 70.0651 & 70.0651 & 0.0000 & 19.75 & 162.1048 & $\mathrm{C}_{11} \mathrm{H}_{14} \mathrm{O}$ \\
\hline \multirow{3}{*}{ 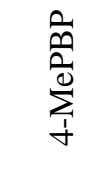 } & $\mathrm{C}_{15} \mathrm{H}_{22} \mathrm{NO}$ & 232.1696 & 232.1682 & 0.0014 & 49.07 & & \\
\hline & $\mathrm{C}_{13} \mathrm{H}_{17} \mathrm{NO}$ & 203.1305 & 203.1257 & 0.0048 & 5.76 & 29.0425 & $\mathrm{C}_{2} \mathrm{H}_{5}$ \\
\hline & $\mathrm{C}_{11} \mathrm{H}_{13} \mathrm{O}$ & 161.0961 & 161.0953 & 0.0008 & 69.09 & 71.0729 & $\mathrm{C}_{4} \mathrm{H}_{9} \mathrm{~N}$ \\
\hline
\end{tabular}




\begin{tabular}{|c|c|c|c|c|c|c|c|}
\hline NPS & $\begin{array}{l}\text { Molecular } \\
\text { formula }\end{array}$ & $\begin{array}{l}\text { Theoretical } \\
\text { mass }(m / z)\end{array}$ & $\begin{array}{r}\text { Measured } \\
\text { mass }(m / z)\end{array}$ & $\begin{array}{c}\text { Mass } \\
\text { difference } \\
(\mathrm{m} / \mathrm{z})\end{array}$ & $\begin{array}{c}\text { Relative } \\
\text { abundance } \\
(\%)\end{array}$ & $\begin{array}{l}\text { Measured } \\
\text { mass loss } \\
(\mathrm{m} / \mathrm{z})\end{array}$ & $\begin{array}{c}\text { Fragment } \\
\text { formula }\end{array}$ \\
\hline & $\mathrm{C}_{10} \mathrm{H}_{13}$ & 133.1012 & 133.1003 & 0.0009 & 36.33 & 99.0679 & $\mathrm{C}_{5} \mathrm{H}_{9} \mathrm{NO}$ \\
\hline & $\mathrm{C}_{8} \mathrm{H}_{7} \mathrm{O}$ & 119.0491 & 119.0483 & 0.0008 & 22.91 & 113.1199 & $\mathrm{C}_{7} \mathrm{H}_{15} \mathrm{~N}$ \\
\hline & $\mathrm{C}_{7} \mathrm{H}_{14} \mathrm{~N}$ & 112.1121 & 112.1117 & 0.0004 & 39.48 & 120.0565 & $\mathrm{C}_{8} \mathrm{H}_{8} \mathrm{O}$ \\
\hline & $\mathrm{C}_{8} \mathrm{H}_{9}$ & 105.0699 & 105.0693 & 0.0006 & 100.00 & 127.0989 & $\mathrm{C}_{7} \mathrm{H}_{13} \mathrm{NO}$ \\
\hline & $\mathrm{C}_{4} \mathrm{H}_{8} \mathrm{~N}$ & 70.0651 & 70.0649 & 0.0002 & 12.48 & 162.1033 & $\mathrm{C}_{11} \mathrm{H}_{14} \mathrm{O}$ \\
\hline \multirow{8}{*}{$\sum_{\frac{1}{d}}^{0}$} & $\mathrm{C}_{15} \mathrm{H}_{22} \mathrm{NO}$ & 232.1696 & 232.1669 & 0.0027 & 70.03 & & \\
\hline & $\mathrm{C}_{12} \mathrm{H}_{15} \mathrm{NO}$ & 189.1148 & 189.1131 & 0.0017 & 6.87 & 43.0538 & $\mathrm{C}_{3} \mathrm{H}_{7}$ \\
\hline & $\mathrm{C}_{11} \mathrm{H}_{13} \mathrm{O}$ & 161.0961 & 161.0936 & 0.0025 & 27.31 & 71.0733 & $\mathrm{C}_{4} \mathrm{H}_{9} \mathrm{~N}$ \\
\hline & $\mathrm{C}_{8} \mathrm{H}_{16} \mathrm{~N}$ & 126.1277 & 126.1251 & 0.0026 & 29.98 & 106.0418 & $\mathrm{C}_{7} \mathrm{H}_{6} \mathrm{O}$ \\
\hline & $\mathrm{C}_{8} \mathrm{H}_{7} \mathrm{O}$ & 119.0491 & 119.0473 & 0.0018 & 12.59 & 113.1196 & $\mathrm{C}_{7} \mathrm{H}_{15} \mathrm{~N}$ \\
\hline & $\mathrm{C}_{7} \mathrm{H}_{5} \mathrm{O}$ & 105.0335 & 105.0323 & 0.0012 & 26.84 & 127.1346 & $\mathrm{C}_{7} \mathrm{H}_{13} \mathrm{NO}$ \\
\hline & $\mathrm{C}_{7} \mathrm{H}_{7}$ & 91.0542 & 91.0532 & 0.0010 & 100.00 & 141.1137 & $\mathrm{C}_{8} \mathrm{H}_{15} \mathrm{NO}$ \\
\hline & $\mathrm{C}_{4} \mathrm{H}_{8} \mathrm{~N}$ & 70.0651 & 70.0648 & 0.0003 & 19.50 & 162.1021 & $\mathrm{C}_{11} \mathrm{H}_{14} \mathrm{O}$ \\
\hline \multirow{6}{*}{$\sum_{\substack{1 \\
\dot{m}}}^{U}$} & $\mathrm{C}_{10} \mathrm{H}_{13} \mathrm{FNO}$ & 182.0976 & 182.0974 & 0.0002 & 1.10 & & \\
\hline & $\mathrm{C}_{10} \mathrm{H}_{11} \mathrm{FN}$ & 164.0870 & 164.0868 & 0.0002 & 51.05 & 18.0106 & $\mathrm{H}_{2} \mathrm{O}$ \\
\hline & $\mathrm{C}_{9} \mathrm{H}_{8} \mathrm{FN}$ & 149.0635 & 149.0637 & -0.0002 & 100.00 & 33.0337 & $\mathrm{CH}_{5} \mathrm{O}$ \\
\hline & $\mathrm{C}_{8} \mathrm{H}_{8} \mathrm{~F}$ & 123.0605 & 123.0601 & 0.0004 & 17.38 & 59.0373 & $\mathrm{C}_{2} \mathrm{H}_{5} \mathrm{NO}$ \\
\hline & $\mathrm{C}_{8} \mathrm{H}_{7}$ & 103.0542 & 103.0536 & 0.0006 & 12.89 & 79.0438 & $\mathrm{C}_{2} \mathrm{H}_{6} \mathrm{FNO}$ \\
\hline & $\mathrm{C}_{6} \mathrm{H}_{5}$ & 77.0386 & 77.0378 & 0.0008 & 2.13 & 105.0596 & $\mathrm{C}_{4} \mathrm{H}_{8} \mathrm{FNO}$ \\
\hline \multirow{6}{*}{ 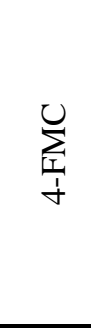 } & $\mathrm{C}_{10} \mathrm{H}_{13} \mathrm{FNO}$ & 182.0976 & 182.0982 & -0.0006 & 0.62 & & \\
\hline & $\mathrm{C}_{10} \mathrm{H}_{11} \mathrm{FN}$ & 164.0870 & 164.0866 & 0.0004 & 51.46 & 18.0116 & $\mathrm{H}_{2} \mathrm{O}$ \\
\hline & $\mathrm{C}_{9} \mathrm{H}_{8} \mathrm{FN}$ & 149.0635 & 149.0630 & 0.0005 & 100.00 & 33.0352 & $\mathrm{CH}_{5} \mathrm{O}$ \\
\hline & $\mathrm{C}_{8} \mathrm{H}_{8} \mathrm{~F}$ & 123.0610 & 123.0597 & 0.0013 & 22.65 & 59.0385 & $\mathrm{C}_{2} \mathrm{H}_{5} \mathrm{NO}$ \\
\hline & $\mathrm{C}_{8} \mathrm{H}_{7}$ & 103.0542 & 103.0541 & 0.0001 & 10.65 & 79.0441 & $\mathrm{C}_{2} \mathrm{H}_{6} \mathrm{FNO}$ \\
\hline & $\mathrm{C}_{6} \mathrm{H}_{5}$ & 77.0386 & 77.0385 & 0.0001 & 2.33 & 105.0597 & $\mathrm{C}_{4} \mathrm{H}_{8} \mathrm{FNO}$ \\
\hline \multirow{7}{*}{$\sum_{j}^{U}$} & $\mathrm{C}_{12} \mathrm{H}_{18} \mathrm{NO}$ & 192.1383 & 192.1390 & -0.0007 & 2.16 & & \\
\hline & $\mathrm{C}_{12} \mathrm{H}_{16} \mathrm{~N}$ & 174.1277 & 174.1274 & 0.0003 & 67.34 & 18.0116 & $\mathrm{H}_{2} \mathrm{O}$ \\
\hline & $\mathrm{C}_{11} \mathrm{H}_{13} \mathrm{~N}$ & 159.1043 & 159.1037 & 0.0006 & 33.09 & 33.0353 & $\mathrm{CH}_{5} \mathrm{O}$ \\
\hline & $\mathrm{C}_{10} \mathrm{H}_{11} \mathrm{~N}$ & 145.0886 & 145.0880 & 0.0006 & 100.00 & 47.0510 & $\mathrm{C}_{2} \mathrm{H}_{7} \mathrm{O}$ \\
\hline & $\mathrm{C}_{10} \mathrm{H}_{11}$ & 131.0861 & 131.0747 & 0.0114 & 23.31 & 61.0643 & $\mathrm{C}_{2} \mathrm{H}_{7} \mathrm{NO}$ \\
\hline & $\mathrm{C}_{9} \mathrm{H}_{11}$ & 119.0855 & 119.0847 & 0.0008 & 35.87 & 73.0543 & $\mathrm{C}_{3} \mathrm{H}_{7} \mathrm{NO}$ \\
\hline & $\mathrm{C}_{7} \mathrm{H}_{7}$ & 91.0542 & 91.0539 & 0.0003 & 9.74 & 101.0851 & $\mathrm{C}_{5} \mathrm{H}_{11} \mathrm{NO}$ \\
\hline
\end{tabular}




\begin{tabular}{|c|c|c|c|c|c|c|c|}
\hline NPS & $\begin{array}{l}\text { Molecular } \\
\text { formula }\end{array}$ & $\begin{array}{l}\text { Theoretical } \\
\text { mass }(m / z)\end{array}$ & $\begin{array}{r}\text { Measured } \\
\text { mass }(m / z)\end{array}$ & $\begin{array}{c}\text { Mass } \\
\text { difference } \\
(\mathrm{m} / \mathrm{z})\end{array}$ & $\begin{array}{c}\text { Relative } \\
\text { abundance } \\
(\%)\end{array}$ & $\begin{array}{l}\text { Measured } \\
\text { mass loss } \\
(\mathrm{m} / \mathrm{z})\end{array}$ & $\begin{array}{c}\text { Fragment } \\
\text { formula }\end{array}$ \\
\hline & $\mathrm{C}_{2} \mathrm{H}_{5} \mathrm{NO}$ & 59.0366 & 59.0492 & -0.0126 & 33.42 & 133.0898 & $\mathrm{C}_{10} \mathrm{H}_{13}$ \\
\hline \multirow{9}{*}{ 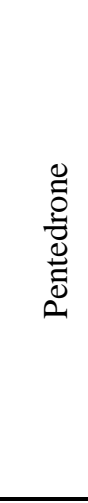 } & $\mathrm{C}_{12} \mathrm{H}_{18} \mathrm{NO}$ & 192.1383 & 192.1372 & 0.0011 & 2.02 & & \\
\hline & $\mathrm{C}_{12} \mathrm{H}_{16} \mathrm{~N}$ & 174.1277 & 174.1269 & 0.0008 & 31.79 & 18.0103 & $\mathrm{H}_{2} \mathrm{O}$ \\
\hline & $\mathrm{C}_{10} \mathrm{H}_{10} \mathrm{~N}$ & 144.0808 & 144.0813 & -0.0005 & 17.64 & 48.0559 & $\mathrm{C}_{2} \mathrm{H}_{8} \mathrm{O}$ \\
\hline & $\mathrm{C}_{10} \mathrm{H}_{12}$ & 132.0934 & 132.0803 & 0.0131 & 100.00 & 60.0569 & $\mathrm{C}_{2} \mathrm{H}_{6} \mathrm{NO}$ \\
\hline & $\mathrm{C}_{9} \mathrm{H}_{9}$ & 117.0699 & 117.0569 & 0.0130 & 14.80 & 75.0803 & $\mathrm{C}_{3} \mathrm{H}_{9} \mathrm{NO}$ \\
\hline & $\mathrm{C}_{8} \mathrm{H}_{9}$ & 105.0699 & 105.0343 & 0.0356 & 16.00 & 87.1029 & $\mathrm{C}_{4} \mathrm{H}_{9} \mathrm{NO}$ \\
\hline & $\mathrm{C}_{7} \mathrm{H}_{7}$ & 91.0542 & 91.0541 & 0.0001 & 75.36 & 101.0831 & $\mathrm{C}_{5} \mathrm{H}_{11} \mathrm{NO}$ \\
\hline & $\mathrm{C}_{6} \mathrm{H}_{5}$ & 77.0386 & 77.0388 & -0.0002 & 4.27 & 115.0984 & $\mathrm{C}_{6} \mathrm{H}_{13} \mathrm{NO}$ \\
\hline & $\mathrm{C}_{2} \mathrm{H}_{5} \mathrm{NO}$ & 59.0366 & 59.0494 & -0.0128 & 55.66 & 133.0878 & $\mathrm{C}_{10} \mathrm{H}_{13}$ \\
\hline \multirow{5}{*}{$\sum_{j}^{U}$} & $\mathrm{C}_{11} \mathrm{H}_{16} \mathrm{NO}$ & 178.1226 & - & & - & & \\
\hline & $\mathrm{C}_{11} \mathrm{H}_{14} \mathrm{~N}$ & 160.1121 & 160.1110 & 0.0011 & 46.86 & 18.0116 & $\mathrm{H}_{2} \mathrm{O}$ \\
\hline & $\mathrm{C}_{10} \mathrm{H}_{11} \mathrm{~N}$ & 145.0886 & 145.0877 & 0.0009 & 100.00 & 33.0349 & $\mathrm{CH}_{5} \mathrm{O}$ \\
\hline & $\mathrm{C}_{9} \mathrm{H}_{11}$ & 119.0855 & 119.0825 & 0.0030 & 15.86 & 59.0401 & $\mathrm{C}_{2} \mathrm{H}_{5} \mathrm{NO}$ \\
\hline & $\mathrm{C}_{7} \mathrm{H}_{7}$ & 91.0542 & 91.0537 & 0.0005 & 6.22 & 87.1226 & $\mathrm{C}_{4} \mathrm{H}_{9} \mathrm{NO}$ \\
\hline \multirow{6}{*}{ 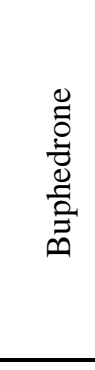 } & $\mathrm{C}_{11} \mathrm{H}_{16} \mathrm{NO}$ & 178.1226 & 178.1198 & 0.0028 & 0.61 & & \\
\hline & $\mathrm{C}_{11} \mathrm{H}_{14} \mathrm{~N}$ & 160.1121 & 160.1109 & 0.0012 & 40.21 & 17.8891 & $\mathrm{H}_{2} \mathrm{O}$ \\
\hline & $\mathrm{C}_{10} \mathrm{H}_{11} \mathrm{~N}$ & 145.0886 & 145.0879 & 0.0007 & 18.26 & 32.9121 & $\mathrm{CH}_{5} \mathrm{O}$ \\
\hline & $\mathrm{C}_{10} \mathrm{H}_{11}$ & 131.0855 & 131.0722 & 0.0133 & 100.00 & 46.9278 & $\mathrm{CH}_{5} \mathrm{NO}$ \\
\hline & $\mathrm{C}_{7} \mathrm{H}_{7}$ & 91.0542 & 91.0544 & -0.0002 & 51.32 & 86.9456 & $\mathrm{C}_{4} \mathrm{H}_{9} \mathrm{NO}$ \\
\hline & $\mathrm{C}_{6} \mathrm{H}_{5}$ & 77.0386 & 77.0392 & -0.0006 & 4.88 & 101.0000 & $\mathrm{C}_{5} \mathrm{H}_{11} \mathrm{NO}$ \\
\hline \multirow{5}{*}{ 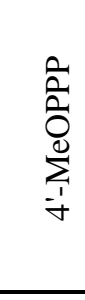 } & $\mathrm{C}_{14} \mathrm{H}_{20} \mathrm{NO}_{2}$ & 234.1489 & 234.1476 & 0.0013 & 42.53 & & \\
\hline & $\mathrm{C}_{10} \mathrm{H}_{11} \mathrm{O}_{2}$ & 163.0754 & 163.0743 & 0.0011 & 60.87 & 71.0733 & $\mathrm{C}_{4} \mathrm{H}_{9} \mathrm{~N}$ \\
\hline & $\mathrm{C}_{8} \mathrm{H}_{7} \mathrm{O}_{2}$ & 135.0441 & 135.0796 & -0.0355 & 59.15 & 99.0680 & $\mathrm{C}_{6} \mathrm{H}_{13} \mathrm{~N}$ \\
\hline & $\mathrm{C}_{6} \mathrm{H}_{12} \mathrm{~N}$ & 98.0964 & 98.0959 & 0.0005 & 100.00 & 136.0517 & $\mathrm{C}_{8} \mathrm{H}_{8} \mathrm{O}_{2}$ \\
\hline & $\mathrm{C}_{4} \mathrm{H}_{8} \mathrm{~N}$ & 70.0651 & 70.0651 & 0.0000 & 3.15 & 164.0825 & $\mathrm{C}_{10} \mathrm{H}_{12} \mathrm{O}_{2}$ \\
\hline \multirow{7}{*}{$\underset{\substack{\mid=1 \\
j}}{\sum_{0}^{0}}$} & $\mathrm{C}_{17} \mathrm{H}_{26} \mathrm{NO}$ & 260.2009 & 260.1999 & 0.0010 & 68.07 & & \\
\hline & $\mathrm{C}_{13} \mathrm{H}_{17} \mathrm{O}$ & 189.1274 & 189.1260 & 0.0014 & 43.13 & 71.0739 & $\mathrm{C}_{4} \mathrm{H}_{9} \mathrm{~N}$ \\
\hline & $\mathrm{C}_{9} \mathrm{H}_{18} \mathrm{~N}$ & 140.1434 & 140.1427 & 0.0007 & 21.71 & 120.0572 & $\mathrm{C}_{8} \mathrm{H}_{8} \mathrm{O}$ \\
\hline & $\mathrm{C}_{10} \mathrm{H}_{13}$ & 133.1012 & 133.0646 & 0.0366 & 11.23 & 127.1353 & $\mathrm{C}_{7} \mathrm{H}_{13} \mathrm{NO}$ \\
\hline & $\mathrm{C}_{8} \mathrm{H}_{7} \mathrm{O}$ & 119.0491 & 119.0487 & 0.0004 & 24.17 & 141.1512 & $\mathrm{C}_{9} \mathrm{H}_{19} \mathrm{~N}$ \\
\hline & $\mathrm{C}_{8} \mathrm{H}_{9}$ & 105.0699 & 105.0693 & 0.0006 & 100.00 & 155.1306 & $\mathrm{C}_{9} \mathrm{H}_{17} \mathrm{NO}$ \\
\hline & $\mathrm{C}_{4} \mathrm{H}_{8} \mathrm{~N}$ & 70.0651 & 70.0649 & 0.0002 & 8.18 & 190.1350 & $\mathrm{C}_{13} \mathrm{H}_{18} \mathrm{O}$ \\
\hline
\end{tabular}




\begin{tabular}{|c|c|c|c|c|c|c|c|}
\hline NPS & $\begin{array}{l}\text { Molecular } \\
\text { formula }\end{array}$ & $\begin{array}{l}\text { Theoretical } \\
\text { mass }(m / z)\end{array}$ & $\begin{array}{r}\text { Measured } \\
\text { mass }(m / z)\end{array}$ & $\begin{array}{c}\text { Mass } \\
\text { difference } \\
(\mathrm{m} / \mathrm{z})\end{array}$ & $\begin{array}{c}\text { Relative } \\
\text { abundance } \\
(\%)\end{array}$ & $\begin{array}{l}\text { Measured } \\
\text { mass loss } \\
(\mathrm{m} / \mathrm{z})\end{array}$ & $\begin{array}{c}\text { Fragment } \\
\text { formula }\end{array}$ \\
\hline \multirow{5}{*}{$\sum_{-1}^{0}$} & $\mathrm{C}_{14} \mathrm{H}_{20} \mathrm{NO}$ & 218.1539 & 218.1524 & 0.0015 & 70.61 & & \\
\hline & $\mathrm{C}_{10} \mathrm{H}_{11} \mathrm{O}$ & 147.0804 & 147.0792 & 0.0012 & 94.18 & 71.0732 & $\mathrm{C}_{4} \mathrm{H}_{9} \mathrm{~N}$ \\
\hline & $\mathrm{C}_{9} \mathrm{H}_{11}$ & 119.0855 & 119.0845 & 0.0010 & 100.00 & 99.0679 & $\mathrm{C}_{6} \mathrm{H}_{13} \mathrm{~N}$ \\
\hline & $\mathrm{C}_{6} \mathrm{H}_{12} \mathrm{~N}$ & 98.0964 & 98.0955 & 0.0009 & 57.47 & 120.0569 & $\mathrm{C}_{8} \mathrm{H}_{8} \mathrm{O}$ \\
\hline & $\mathrm{C}_{4} \mathrm{H}_{8} \mathrm{~N}$ & 70.0651 & 70.0645 & 0.0006 & 15.80 & 148.0879 & $\mathrm{C}_{10} \mathrm{H}_{12} \mathrm{O}$ \\
\hline \multirow{6}{*}{$\sum_{\substack{i \\
0}}^{\sum_{0}^{\mid}}$} & $\mathrm{C}_{12} \mathrm{H}_{16} \mathrm{NO}_{3}$ & 222.1125 & 222.1126 & -0.0001 & 20.36 & & \\
\hline & $\mathrm{C}_{10} \mathrm{H}_{9} \mathrm{O}_{3}$ & 177.0546 & 177.0546 & 0.0000 & 17.77 & 45.0580 & $\mathrm{C}_{2} \mathrm{H}_{7} \mathrm{~N}$ \\
\hline & $\mathrm{C}_{9} \mathrm{H}_{7} \mathrm{O}_{2}$ & 147.0441 & 147.0441 & 0.0000 & 65.88 & 75.0685 & $\mathrm{C}_{3} \mathrm{H}_{9} \mathrm{NO}$ \\
\hline & $\mathrm{C}_{8} \mathrm{H}_{7} \mathrm{O}$ & 119.0855 & 119.0485 & 0.0370 & 11.65 & 103.0641 & $\mathrm{C}_{3} \mathrm{H}_{5} \mathrm{NO}_{3}$ \\
\hline & $\mathrm{C}_{7} \mathrm{H}_{7}$ & 91.0542 & 91.0546 & -0.0004 & 7.00 & 131.0580 & $\mathrm{C}_{5} \mathrm{H}_{9} \mathrm{NO}_{3}$ \\
\hline & $\mathrm{C}_{4} \mathrm{H}_{10} \mathrm{~N}$ & 72.0808 & 72.0813 & -0.0005 & 100.00 & 150.0313 & $\mathrm{C}_{8} \mathrm{H}_{6} \mathrm{O}_{3}$ \\
\hline \multirow{8}{*}{ 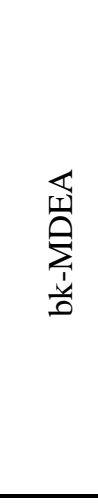 } & $\mathrm{C}_{12} \mathrm{H}_{16} \mathrm{NO}_{3}$ & 222.1125 & 222.1121 & 0.0004 & 2.43 & & \\
\hline & $\mathrm{C}_{12} \mathrm{H}_{14} \mathrm{NO}_{2}$ & 204.1019 & 204.1014 & 0.0005 & 21.28 & 18.0107 & $\mathrm{H}_{2} \mathrm{O}$ \\
\hline & $\mathrm{C}_{11} \mathrm{H}_{11} \mathrm{NO}_{2}$ & 189.0784 & 189.0773 & 0.0011 & 4.81 & 33.1121 & $\mathrm{CH}_{5} \mathrm{O}$ \\
\hline & $\mathrm{C}_{11} \mathrm{H}_{10} \mathrm{O}_{2}$ & 174.0555 & 174.0913 & -0.0358 & 100.00 & 48.0208 & $\mathrm{CH}_{6} \mathrm{NO}$ \\
\hline & $\mathrm{C}_{10} \mathrm{H}_{12} \mathrm{~N}$ & 146.0362 & 146.0958 & -0.0596 & 16.46 & 76.0163 & $\mathrm{C}_{2} \mathrm{H}_{4} \mathrm{O}_{3}$ \\
\hline & $\mathrm{C}_{8} \mathrm{H}_{7} \mathrm{O}$ & 119.0855 & 119.0501 & 0.0354 & 2.46 & 103.0620 & $\mathrm{C}_{4} \mathrm{H}_{9} \mathrm{NO}_{2}$ \\
\hline & $\mathrm{C}_{7} \mathrm{H}_{7}$ & 91.0542 & 91.0535 & 0.0007 & 2.53 & 131.0586 & $\mathrm{C}_{5} \mathrm{H}_{9} \mathrm{NO}_{3}$ \\
\hline & $\mathrm{C}_{4} \mathrm{H}_{10} \mathrm{~N}$ & 72.0808 & 72.0809 & -0.0001 & 12.52 & 150.0312 & $\mathrm{C}_{8} \mathrm{H}_{6} \mathrm{O}_{3}$ \\
\hline \multirow{7}{*}{ 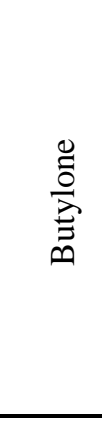 } & $\mathrm{C}_{12} \mathrm{H}_{16} \mathrm{NO}_{3}$ & 222.1125 & 222.1116 & 0.0009 & 0.93 & & \\
\hline & $\mathrm{C}_{12} \mathrm{H}_{14} \mathrm{NO}_{2}$ & 204.1019 & 204.1016 & 0.0003 & 19.06 & 18.0100 & $\mathrm{H}_{2} \mathrm{O}$ \\
\hline & $\mathrm{C}_{11} \mathrm{H}_{10} \mathrm{O}_{2}$ & 174.0675 & 174.0915 & -0.0240 & 100.00 & 48.0201 & $\mathrm{CH}_{6} \mathrm{NO}$ \\
\hline & $\mathrm{C}_{10} \mathrm{H}_{9} \mathrm{O}_{2}$ & 161.0597 & 161.0599 & -0.0002 & 13.86 & 61.0517 & $\mathrm{C}_{2} \mathrm{H}_{7} \mathrm{NO}$ \\
\hline & $\mathrm{C}_{10} \mathrm{H}_{12} \mathrm{~N}$ & 146.0964 & 146.0960 & 0.0004 & 18.87 & 76.0156 & $\mathrm{C}_{2} \mathrm{H}_{4} \mathrm{O}_{3}$ \\
\hline & $\mathrm{C}_{8} \mathrm{H}_{9}$ & 105.0699 & 105.0699 & 0.0000 & 3.95 & 117.0417 & $\mathrm{C}_{4} \mathrm{H}_{7} \mathrm{NO}_{3}$ \\
\hline & $\mathrm{C}_{4} \mathrm{H}_{10} \mathrm{~N}$ & 72.0808 & 72.0816 & -0.0008 & 16.81 & 150.0300 & $\mathrm{C}_{8} \mathrm{H}_{6} \mathrm{O}_{3}$ \\
\hline \multirow{6}{*}{ 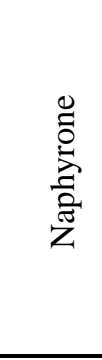 } & $\mathrm{C}_{19} \mathrm{H}_{24} \mathrm{NO}$ & 282.1852 & 282.1854 & -0.0002 & 93.08 & & \\
\hline & $\mathrm{C}_{15} \mathrm{H}_{15} \mathrm{O}$ & 211.1117 & 211.1122 & -0.0005 & 82.03 & 71.0732 & $\mathrm{C}_{4} \mathrm{H}_{9} \mathrm{~N}$ \\
\hline & $\mathrm{C}_{11} \mathrm{H}_{7} \mathrm{O}$ & 155.0491 & 155.0481 & 0.0010 & 17.42 & 127.1373 & $\mathrm{C}_{8} \mathrm{H}_{17} \mathrm{~N}$ \\
\hline & $\mathrm{C}_{11} \mathrm{H}_{9}$ & 141.0699 & 141.0698 & 0.0001 & 100.00 & 141.1156 & $\mathrm{C}_{8} \mathrm{H}_{15} \mathrm{NO}$ \\
\hline & $\mathrm{C}_{8} \mathrm{H}_{16} \mathrm{~N}$ & 126.1277 & 126.1277 & 0.0000 & 28.35 & 156.0577 & $\mathrm{C}_{11} \mathrm{H}_{8} \mathrm{O}$ \\
\hline & $\mathrm{C}_{4} \mathrm{H}_{8} \mathrm{~N}$ & 70.0651 & 70.0651 & 0.0000 & 12.40 & 212.1203 & $\mathrm{C}_{15} \mathrm{H}_{16} \mathrm{O}$ \\
\hline
\end{tabular}




\begin{tabular}{|c|c|c|c|c|c|c|c|}
\hline NPS & $\begin{array}{l}\text { Molecular } \\
\text { formula }\end{array}$ & $\begin{array}{l}\text { Theoretical } \\
\text { mass }(m / z)\end{array}$ & $\begin{array}{r}\text { Measured } \\
\text { mass }(m / z)\end{array}$ & $\begin{array}{c}\text { Mass } \\
\text { difference } \\
(m / z)\end{array}$ & $\begin{array}{c}\text { Relative } \\
\text { abundance } \\
(\%)\end{array}$ & $\begin{array}{l}\text { Measured } \\
\text { mass loss } \\
\quad(\mathrm{m} / \mathrm{z})\end{array}$ & $\begin{array}{c}\text { Fragment } \\
\text { formula }\end{array}$ \\
\hline \multirow{6}{*}{ 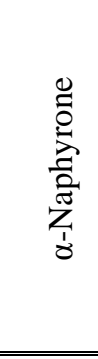 } & $\mathrm{C}_{19} \mathrm{H}_{24} \mathrm{NO}$ & 282.1852 & 282.1857 & -0.0005 & 100.00 & & \\
\hline & $\mathrm{C}_{15} \mathrm{H}_{15} \mathrm{O}$ & 211.1117 & 211.1121 & -0.0004 & 76.52 & 71.0736 & $\mathrm{C}_{4} \mathrm{H}_{9} \mathrm{~N}$ \\
\hline & $\mathrm{C}_{11} \mathrm{H}_{7} \mathrm{O}$ & 155.0491 & 155.0489 & 0.0002 & 30.86 & 127.1368 & $\mathrm{C}_{8} \mathrm{H}_{17} \mathrm{~N}$ \\
\hline & $\mathrm{C}_{11} \mathrm{H}_{9}$ & 141.0699 & 141.0697 & 0.0002 & 58.06 & 141.1160 & $\mathrm{C}_{8} \mathrm{H}_{15} \mathrm{NO}$ \\
\hline & $\mathrm{C}_{8} \mathrm{H}_{16} \mathrm{~N}$ & 126.1277 & 126.1276 & 0.0001 & 74.75 & 156.0581 & $\mathrm{C}_{11} \mathrm{H}_{8} \mathrm{O}$ \\
\hline & $\mathrm{C}_{4} \mathrm{H}_{8} \mathrm{~N}$ & 70.0651 & 70.0653 & -0.0002 & 6.84 & 212.1204 & $\mathrm{C}_{15} \mathrm{H}_{16} \mathrm{O}$ \\
\hline \multicolumn{8}{|c|}{ Synthetic cannabinoids } \\
\hline \multirow{6}{*}{ 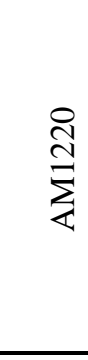 } & $\mathrm{C}_{26} \mathrm{H}_{27} \mathrm{~N}_{2} \mathrm{O}$ & 383.2118 & 383.2110 & 0.0008 & 44.71 & & \\
\hline & $\mathrm{C}_{20} \mathrm{H}_{16} \mathrm{NO}$ & 286.1226 & 286.1211 & 0.0015 & 31.51 & 97.0899 & $\mathrm{C}_{6} \mathrm{H}_{11} \mathrm{~N}$ \\
\hline & $\mathrm{C}_{11} \mathrm{H}_{7} \mathrm{O}$ & 155.0491 & 155.0484 & 0.0007 & 40.45 & 228.1626 & $\mathrm{C}_{15} \mathrm{H}_{20} \mathrm{~N}_{2}$ \\
\hline & $\mathrm{C}_{7} \mathrm{H}_{14} \mathrm{~N}$ & 112.1121 & 112.1116 & 0.0005 & 100.00 & 271.0994 & $\mathrm{C}_{19} \mathrm{H}_{13} \mathrm{NO}$ \\
\hline & $\mathrm{C}_{6} \mathrm{H}_{12} \mathrm{~N}$ & 98.0964 & 98.0962 & 0.0002 & 95.52 & 285.1148 & $\mathrm{C}_{20} \mathrm{H}_{15} \mathrm{NO}$ \\
\hline & $\mathrm{C}_{4} \mathrm{H}_{8} \mathrm{~N}$ & 70.0651 & 70.0641 & 0.0010 & 3.96 & 313.1469 & $\mathrm{C}_{22} \mathrm{H}_{19} \mathrm{NO}$ \\
\hline \multirow{6}{*}{ 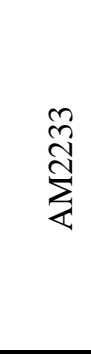 } & $\mathrm{C}_{22} \mathrm{H}_{24} \mathrm{IN}_{2} \mathrm{O}$ & 459.0928 & 459.0909 & 0.0019 & 100.00 & & \\
\hline & $\mathrm{C}_{16} \mathrm{H}_{13} \mathrm{INO}$ & 362.0036 & 362.0020 & 0.0016 & 35.40 & 97.0889 & $\mathrm{C}_{6} \mathrm{H}_{11} \mathrm{~N}$ \\
\hline & $\mathrm{C}_{7} \mathrm{H}_{4} \mathrm{IO}$ & 230.9301 & 230.9286 & 0.0015 & 22.29 & 228.1623 & $\mathrm{C}_{15} \mathrm{H}_{20} \mathrm{~N}_{2}$ \\
\hline & $\mathrm{C}_{7} \mathrm{H}_{14} \mathrm{~N}$ & 112.1126 & 112.1115 & 0.0011 & 77.23 & 346.9794 & $\mathrm{C}_{15} \mathrm{H}_{10} \mathrm{INO}$ \\
\hline & $\mathrm{C}_{6} \mathrm{H}_{12} \mathrm{~N}$ & 98.0964 & 98.0959 & 0.0005 & 97.31 & 360.9950 & $\mathrm{C}_{16} \mathrm{H}_{12} \mathrm{INO}$ \\
\hline & $\mathrm{C}_{4} \mathrm{H}_{8} \mathrm{~N}$ & 70.0651 & 70.0647 & 0.0004 & 2.68 & 389.0262 & $\mathrm{C}_{18} \mathrm{H}_{16} \mathrm{INO}$ \\
\hline \multirow{6}{*}{ 崩 } & $\mathrm{C}_{24} \mathrm{H}_{29} \mathrm{~N}_{2} \mathrm{O}_{2}$ & 377.2224 & 377.2203 & 0.0021 & 63.77 & & \\
\hline & $\mathrm{C}_{18} \mathrm{H}_{18} \mathrm{NO}_{2}$ & 280.1332 & 280.1311 & 0.0021 & 11.56 & 97.0892 & $\mathrm{C}_{6} \mathrm{H}_{11} \mathrm{~N}$ \\
\hline & $\mathrm{C}_{15} \mathrm{H}_{21} \mathrm{~N}_{2}$ & 229.1699 & 229.1681 & 0.0018 & 28.59 & 148.0522 & $\mathrm{C}_{9} \mathrm{H}_{8} \mathrm{O}_{2}$ \\
\hline & $\mathrm{C}_{8} \mathrm{H}_{9} \mathrm{O}$ & 121.0648 & 121.0636 & 0.0012 & 62.24 & 256.1567 & $\mathrm{C}_{16} \mathrm{H}_{20} \mathrm{~N}_{2} \mathrm{O}$ \\
\hline & $\mathrm{C}_{7} \mathrm{H}_{14} \mathrm{~N}$ & 112.1121 & 112.1114 & 0.0007 & 100.00 & 265.1089 & $\mathrm{C}_{17} \mathrm{H}_{15} \mathrm{NO}_{2}$ \\
\hline & $\mathrm{C}_{6} \mathrm{H}_{12} \mathrm{~N}$ & 98.0964 & 98.0954 & 0.0010 & 32.35 & 279.1249 & $\mathrm{C}_{18} \mathrm{H}_{17} \mathrm{NO}_{2}$ \\
\hline \multirow{4}{*}{ 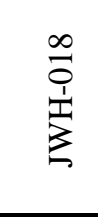 } & $\mathrm{C}_{{ }_{4}} \mathrm{H}_{3}{ }_{4} \mathrm{NO}$ & 342.1852 & 342.1826 & 0.0026 & 75.63 & & \\
\hline & $\mathrm{C}_{14} \mathrm{H}_{16} \mathrm{NO}$ & 214.1226 & 214.1210 & 0.0016 & 19.72 & 128.0616 & $\mathrm{C}_{10} \mathrm{H}_{8}$ \\
\hline & $\mathrm{C}_{11} \mathrm{H}_{7} \mathrm{O}$ & 155.0491 & 155.0477 & 0.0014 & 100.00 & 187.1349 & $\mathrm{C}_{13} \mathrm{H}_{17} \mathrm{~N}$ \\
\hline & $\mathrm{C}_{10} \mathrm{H}_{7}$ & 127.0542 & 127.0525 & 0.0017 & 6.81 & 215.1301 & $\mathrm{C}_{14} \mathrm{H}_{17} \mathrm{NO}$ \\
\hline \multirow{4}{*}{ 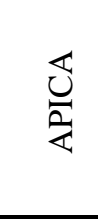 } & $\mathrm{C}_{34} \mathrm{H}_{33} \mathrm{~N}_{2} \mathrm{O}$ & 365.2587 & 365.2561 & 0.0026 & 100.00 & & \\
\hline & $\mathrm{C}_{14} \mathrm{H}_{16} \mathrm{NO}$ & 214.1226 & 214.1218 & 0.0008 & 15.49 & 151.1343 & $\mathrm{C}_{10} \mathrm{H}_{17} \mathrm{~N}$ \\
\hline & $\mathrm{C}_{10} \mathrm{H}_{15}$ & 135.1168 & 135.1157 & 0.0011 & 50.50 & 230.1404 & $\mathrm{C}_{14} \mathrm{H}_{18} \mathrm{~N}_{2} \mathrm{O}$ \\
\hline & $\mathrm{C}_{8} \mathrm{H}_{11}$ & 107.0861 & 107.0836 & 0.0025 & 2.33 & 258.1725 & $\mathrm{C}_{16} \mathrm{H}_{22} \mathrm{~N}_{2} \mathrm{O}$ \\
\hline
\end{tabular}




\begin{tabular}{|c|c|c|c|c|c|c|c|}
\hline NPS & $\begin{array}{l}\text { Molecular } \\
\text { formula }\end{array}$ & $\begin{array}{l}\text { Theoretical } \\
\text { mass }(m / z)\end{array}$ & $\begin{array}{r}\text { Measured } \\
\text { mass }(m / z)\end{array}$ & $\begin{array}{c}\text { Mass } \\
\text { difference } \\
\quad(m / z)\end{array}$ & $\begin{array}{l}\text { Relative } \\
\text { abundance } \\
(\%)\end{array}$ & $\begin{array}{c}\text { Measured } \\
\text { mass loss } \\
(m / z)\end{array}$ & $\begin{array}{l}\text { Fragment } \\
\text { formula }\end{array}$ \\
\hline \multirow{4}{*}{ 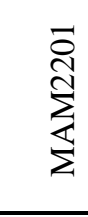 } & $\mathrm{C}_{25} \mathrm{H}_{5} \mathrm{FNO}$ & 374.1915 & 374.1897 & 0.0018 & 100.00 & & \\
\hline & $\mathrm{C}_{14} \mathrm{H}_{15} \mathrm{FNO}$ & 232.1132 & 232.1116 & 0.0016 & 28.40 & 142.0781 & $\mathrm{C}_{11} \mathrm{H}_{10}$ \\
\hline & $\mathrm{C}_{12} \mathrm{H}_{9} \mathrm{O}$ & 169.0648 & 169.0638 & 0.0010 & 90.30 & 205.1259 & $\mathrm{C}_{13} \mathrm{H}_{16} \mathrm{FN}$ \\
\hline & $\mathrm{C}_{11} \mathrm{H}_{9}$ & 141.0699 & 141.0673 & 0.0026 & 4.23 & 233.1224 & $\mathrm{C}_{14} \mathrm{H}_{16} \mathrm{FNO}$ \\
\hline \multirow{6}{*}{$\begin{array}{l}\frac{J}{d} \\
\stackrel{2}{s}\end{array}$} & $\mathrm{C}_{21} \mathrm{H}_{30} \mathrm{NO}$ & 312.2322 & 312.2310 & 0.0012 & 100.00 & & \\
\hline & $\mathrm{C}_{21} \mathrm{H}_{28} \mathrm{~N}$ & 294.2216 & 294.2217 & -0.0001 & 10.38 & 18.0093 & $\mathrm{H}_{2} \mathrm{O}$ \\
\hline & $\mathrm{C}_{14} \mathrm{H}_{16} \mathrm{NO}$ & 214.1226 & 214.1198 & 0.0028 & 28.33 & 98.1112 & $\mathrm{C}_{7} \mathrm{H}_{14}$ \\
\hline & $\mathrm{C}_{9} \mathrm{H}_{6} \mathrm{NO}$ & 144.0444 & 144.0435 & 0.0009 & 1.97 & 168.1875 & $\mathrm{C}_{12} \mathrm{H}_{24}$ \\
\hline & $\mathrm{C}_{8} \mathrm{H}_{13} \mathrm{O}$ & 125.0961 & 125.0951 & 0.0010 & 78.29 & 187.1359 & $\mathrm{C}_{13} \mathrm{H}_{17} \mathrm{O}$ \\
\hline & $\mathrm{C}_{7} \mathrm{H}_{13}$ & 97.1012 & 97.0997 & 0.0015 & 9.10 & 215.1313 & $\mathrm{C}_{14} \mathrm{H}_{17} \mathrm{NO}$ \\
\hline \multirow{4}{*}{$\begin{array}{l}\bar{z} \\
\frac{a}{2} \\
x\end{array}$} & $\mathrm{C}_{2,1} \mathrm{H}_{29} \mathrm{FNO}$ & 330.2228 & 330.2202 & 0.0026 & 100.00 & & \\
\hline & $\mathrm{C}_{21} \mathrm{H}_{27} \mathrm{FN}$ & 312.2122 & 312.2101 & 0.0021 & 9.76 & 18.0101 & $\mathrm{H}_{2} \mathrm{O}$ \\
\hline & $\mathrm{C}_{14} \mathrm{H}_{15} \mathrm{FNO}$ & 232.1132 & 232.1117 & 0.0015 & 17.84 & 98.1085 & $\mathrm{C}_{7} \mathrm{H}_{14}$ \\
\hline & $\mathrm{C}_{8} \mathrm{H}_{13} \mathrm{O}$ & 125.0961 & 125.0953 & 0.0008 & 51.38 & 205.1249 & $\mathrm{C}_{13} \mathrm{H}_{16} \mathrm{FN}$ \\
\hline \multicolumn{8}{|c|}{ Phenethylamines } \\
\hline \multirow{4}{*}{$\begin{array}{l}\sum_{0}^{0} \\
\sum_{1}^{n} \\
\stackrel{1}{n}\end{array}$} & $\mathrm{C}_{18} \mathrm{H}_{23} \mathrm{INO}_{3}$ & 428.0717 & 428.0681 & 0.0036 & 11.60 & & \\
\hline & $\mathrm{C}_{16} \mathrm{H}_{18} \mathrm{NO}_{3}$ & 272.1281 & 272.1387 & -0.0106 & 3.81 & 155.9294 & $\mathrm{C}_{2} \mathrm{H}_{5} \mathrm{I}$ \\
\hline & $\mathrm{C}_{8} \mathrm{H}_{9} \mathrm{O}$ & 121.0648 & 121.0640 & 0.0008 & 100.00 & 307.0041 & $\mathrm{C}_{10} \mathrm{H}_{14} \mathrm{INO}_{2}$ \\
\hline & $\mathrm{C}_{7} \mathrm{H}_{7}$ & 91.0542 & 91.0536 & 0.0006 & 9.20 & 337.0145 & $\mathrm{C}_{11} \mathrm{H}_{16} \mathrm{INO}_{3}$ \\
\hline
\end{tabular}


Appendix 3. Optimized EI MRM transitions for 34 compounds of interest with the average relative abundance (\%) and selected collision energy (CE, V). The most abundant ions are in bold.

\begin{tabular}{|c|c|c|c|c|c|c|c|c|c|c|c|}
\hline Analy te & $\begin{array}{l}\text { Precursor } \\
\text { Ion }\end{array}$ & 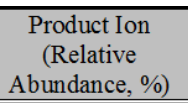 & $\mathrm{CE}(\mathrm{V})$ & Analy te & $\begin{array}{l}\text { Precursor } \\
\text { Ion }\end{array}$ & $\begin{array}{c}\text { Product Ion } \\
\text { (Relative } \\
\text { Abundance, \%) } \\
\end{array}$ & $\mathrm{CE}(\mathrm{V})$ & Analyte & $\begin{array}{l}\text { Precursor } \\
\text { Ion }\end{array}$ & $\begin{array}{c}\text { Product Ion } \\
\text { (Relative } \\
\text { Abundance, \%) } \\
\end{array}$ & $\mathrm{CE}(\mathrm{V})$ \\
\hline 3-FMC & 58 & $30(100)$ & 8 & JWH-250 & 214 & $144(100)$ & 12 & AM2201 & 232 & $144(100)$ & 12 \\
\hline 3-FMC & 58 & $42(74)$ & 20 & JWH-250 & 214 & $116(44)$ & 28 & AM2201 & 359 & $284(42)$ & 12 \\
\hline 3-FMC & 58 & $56(37)$ & 12 & JWH-250 & 335 & $214(5)$ & 4 & AM2201 & 359 & $232(13)$ & 20 \\
\hline 4-MMC & 58 & $30(100)$ & 8 & JWH-302 & 214 & $144(100)$ & 12 & CPE & 98 & $70(100)$ & 12 \\
\hline 4-MMC & 58 & $42(74)$ & 20 & JWH-302 & 214 & $116(46)$ & 28 & CPE & 98 & $42(81)$ & 24 \\
\hline 4-MMC & 58 & $56(40)$ & 12 & JWH-302 & 335 & $214(5)$ & 4 & CPE & 98 & $68(12)$ & 24 \\
\hline 4-MEC & 72 & $44(100)$ & 8 & RCS-4 & 321 & $264(100)$ & 12 & JWH-122 & 298 & $181(100)$ & 8 \\
\hline \multirow[t]{2}{*}{ 4-MEC } & 72 & $29(15)$ & 16 & RCS-4 & 321 & $135(74)$ & 32 & JWH-122 & 355 & $298(62)$ & 8 \\
\hline & & & & RCS-4 & 321 & $186(62)$ & 12 & JWH-122 & 355 & $181(53)$ & 20 \\
\hline 3,4-DMMC & 58 & $30(100)$ & 8 & JWH-249 & 214 & $144(100)$ & 12 & RCS-8 & 254 & $69(100)$ & 20 \\
\hline 3,4-DMMC & 58 & $42(74)$ & 20 & JWH-249 & 214 & $116(45)$ & 28 & RCS-8 & 254 & $144(90)$ & 12 \\
\hline 3,4-DMMC & 58 & $56(41)$ & 12 & JWH-249 & 383 & $214(2)$ & 4 & RCS-8 & 254 & $158(32)$ & 12 \\
\hline Methedrone & 58 & $30(100)$ & 8 & JWH-201 & 214 & $144(100)$ & 12 & JWH-398 & 214 & $144(100)$ & 8 \\
\hline Methedrone & 58 & $42(74)$ & 20 & JWH-201 & 214 & $116(45)$ & 28 & JWH-398 & 375 & $318(33)$ & 8 \\
\hline Methedrone & 58 & $56(40)$ & 12 & & & & & JWH-398 & 375 & $358(21)$ & 12 \\
\hline Methylone & 58 & $30(100)$ & 8 & AM694 & 232 & $144(100)$ & 12 & AM2233 & 98 & $70(100)$ & 12 \\
\hline Methylone & 58 & $42(73)$ & 20 & AM694 & 232 & $116(54)$ & 32 & AM2233 & 98 & $42(82)$ & 28 \\
\hline Methylone & 58 & $56(39)$ & 12 & AM694 & 435 & $232(44)$ & 4 & AM2233 & 98 & $68(12)$ & 24 \\
\hline MDPV & 126 & $69(100)$ & 20 & JWH-073 & 200 & $144(100)$ & 8 & CB-13 & 171 & $115(100)$ & 24 \\
\hline MDPV & 126 & $97(98)$ & 12 & JWH-073 & 200 & $116(48)$ & 24 & CB-13 & 171 & $143(53)$ & 8 \\
\hline MDPV & 126 & $84(96)$ & 12 & JWH-073 & 327 & $310(40)$ & 8 & CB-13 & 368 & $171(17)$ & 24 \\
\hline CP-47,497 & 215 & $81(100)$ & 16 & APINACA & 215 & $145(100)$ & 12 & APICA & 214 & $144(100)$ & 12 \\
\hline CP-47,497 & 215 & $147(33)$ & 12 & APINACA & 217 & $90(42)$ & 36 & APICA & 214 & $116(43)$ & 28 \\
\hline CP-47,497 & 318 & $215(16)$ & 12 & APINACA & 365 & $215(5)$ & 24 & APICA & 364 & 307 (11) & 8 \\
\hline XLR-11 & 232 & $144(100)$ & 12 & JWH-022 & 155 & $127(100)$ & 12 & JWH-081 & 214 & $144(100)$ & 8 \\
\hline XLR-11 & 329 & $247(2)$ & 8 & JWH-022 & 339 & $155(9)$ & 20 & JWH-081 & 371 & $354(43)$ & 12 \\
\hline XLR-11 & 329 & 314 (1.6) & 12 & JWH-022 & 339 & $184(8.5)$ & 4 & JWH-081 & 371 & $214(10)$ & 28 \\
\hline CP-47,497 C8 & 215 & $81(100)$ & 16 & JWH-018 & 214 & $144(100)$ & 8 & AM1220 & 98 & $70(100)$ & 12 \\
\hline CP-47,497 C8 & 215 & $147(33)$ & 12 & JWH-018 & 341 & $284(48)$ & 8 & AM1220 & 98 & $42(82)$ & 28 \\
\hline CP-47,497 C8 & 332 & 215 (14) & 12 & JWH-018 & 341 & $167(27)$ & 20 & AM1220 & 98 & $68(12)$ & 24 \\
\hline JWH-251 & 214 & $144(100)$ & 12 & JWH-019 & 228 & $144(100)$ & 8 & JWH-200 & 100 & $56(100)$ & 12 \\
\hline JWH-251 & 214 & $116(45)$ & 28 & JWH-019 & 355 & $284(51)$ & 12 & JWH-200 & 100 & $70(52)$ & 12 \\
\hline JWH-251 & 319 & $214(7)$ & 4 & JWH-019 & 355 & $338(43)$ & 8 & JWH-200 & 100 & $44(17)$ & 12 \\
\hline JWH-203 & 214 & $144(100)$ & 12 & & & & & & & & \\
\hline JWH-203 & 214 & $116(45)$ & 28 & & & & & & & & \\
\hline JWH-203 & 339 & $214(4)$ & 4 & & & & & & & & \\
\hline
\end{tabular}


Appendix 4. Optimized CI MRM transitions for 34 compounds of interest with the average relative abundance $(\%)$ and selected collision energy (CE, V). The most abundant ions are in bold.

\begin{tabular}{|c|c|c|c|c|c|c|c|c|c|c|c|}
\hline Analy te & $\begin{array}{l}\text { Precursor } \\
\text { Ion }\end{array}$ & $\begin{array}{c}\text { Product Ion } \\
\text { (Relative } \\
\text { Abundance, \%) } \\
\end{array}$ & $\mathrm{CE}(\mathrm{V})$ & Analy te & $\begin{array}{l}\text { Precursor } \\
\text { Ion }\end{array}$ & $\begin{array}{c}\text { Product Ion } \\
\text { (Relative } \\
\text { Abundance, \%) } \\
\end{array}$ & $\mathrm{CE}(\mathrm{V})$ & Analyte & $\begin{array}{l}\text { Precursor } \\
\text { Ion }\end{array}$ & $\begin{array}{c}\text { Product Ion } \\
\text { (Relative } \\
\text { Abundance, \%) } \\
\end{array}$ & $\mathrm{CE}(\mathrm{V})$ \\
\hline 3-FMC & 182 & $164(100)$ & 8 & JWH-250 & 336 & $121(100)$ & 20 & AM2201 & 360 & $\mathbf{1 5 5}(100)$ & 24 \\
\hline 3-FMC & 182 & $149(61)$ & 20 & JWH-250 & 336 & $200(8)$ & 20 & AM2201 & 360 & $232(25)$ & 24 \\
\hline 3-FMC & 182 & $103(20)$ & 32 & JWH-250 & 336 & $188(6)$ & 12 & AM2201 & 360 & $127(95)$ & 56 \\
\hline 4-MMC & 178 & $160(100)$ & 8 & JWH-302 & 336 & $121(100)$ & 20 & CPE & 377 & $112(100)$ & 20 \\
\hline 4-MMC & 178 & $145(58)$ & 20 & JWH-302 & 336 & $214(62)$ & 24 & CPE & 377 & $229(17)$ & 12 \\
\hline 4-MMC & 178 & 119 (11) & 24 & JWH-302 & 336 & $144(29)$ & 44 & CPE & 377 & $121(50)$ & 24 \\
\hline 4-MEC & 192 & $174(100)$ & 8 & RCS-4 & 322 & $135(100)$ & 24 & JWH-122 & 356 & $169(100)$ & 24 \\
\hline 4-MEC & 192 & $145(36)$ & 20 & RCS-4 & 322 & $214(7)$ & 24 & JWH-122 & 356 & $214(33)$ & 20 \\
\hline 4-MEC & 192 & $131(15)$ & 28 & RCS-4 & 322 & $107(20)$ & 44 & JWH-122 & 356 & $141(62)$ & 48 \\
\hline 3,4-DMMC & 192 & $174(100)$ & 12 & JWH-249 & 384 & $169(100)$ & 32 & RCS-8 & 376 & $121(100)$ & 24 \\
\hline 3,4-DMMC & 192 & $159(73)$ & 20 & JWH-249 & 384 & $214(31)$ & 24 & RCS-8 & 376 & $228(5)$ & 12 \\
\hline 3,4-DMMC & 192 & $144(18)$ & 36 & JWH-249 & 384 & $188(19)$ & 16 & RCS-8 & 376 & $144(9)$ & 40 \\
\hline Methedrone & 194 & $176(100)$ & 8 & JWH-201 & 336 & $121(100)$ & 28 & JWH-398 & 376 & $189(100)$ & 24 \\
\hline Methedrone & 194 & $161(36)$ & 20 & JWH-201 & 336 & 149 (24) & 20 & JWH-398 & 376 & 214 (12) & 24 \\
\hline Methedrone & 194 & $135(9)$ & 20 & JWH-201 & 336 & $135(31)$ & 28 & JWH-398 & 376 & $161(50)$ & 52 \\
\hline Methylone & 208 & $160(100)$ & 16 & AM694 & 436 & $231(100)$ & 28 & AM2233 & 459 & $98(100)$ & 36 \\
\hline Methylone & 208 & $190(67)$ & 8 & AM694 & 436 & $309(6)$ & 16 & AM2233 & 459 & $362(7)$ & 16 \\
\hline Methylone & 208 & $132(48)$ & 32 & & & & & AM2233 & 459 & 231 (17) & 32 \\
\hline MDPV & 276 & $\mathbf{1 2 6}(100)$ & 24 & JWH-073 & 328 & $\mathbf{1 5 5}(100)$ & 24 & CB-13 & 369 & 299 (100) & 12 \\
\hline MDPV & 276 & $205(58)$ & 16 & JWH-073 & 328 & 200 (28) & 20 & CB-13 & 369 & $241(36)$ & 12 \\
\hline MDPV & 276 & $175(68)$ & 20 & & & & & & & & \\
\hline CP-47,497 & 301 & $233(100)$ & 8 & APINACA & 366 & $135(100)$ & 16 & APICA & 365 & 214 (100) & 20 \\
\hline CP-47,497 & 301 & $216(7)$ & 12 & APINACA & 366 & 215 (11) & 20 & APICA & 365 & $188(17)$ & 20 \\
\hline CP-47,497 & 301 & $121(33)$ & 32 & & & & & & & & \\
\hline XLR-11 & 330 & $125(100)$ & 20 & JWH-022 & 340 & $\mathbf{1 5 5}(100)$ & 24 & JWH-081 & 372 & $185(100)$ & 24 \\
\hline XLR-11 & 330 & $232(46)$ & 20 & JWH-022 & 340 & $212(24)$ & 20 & JWH-081 & 372 & $214(34)$ & 20 \\
\hline XLR-11 & 330 & $97(23)$ & 28 & & & & & JWH-081 & 372 & 157 (24) & 44 \\
\hline CP-47,497 C8 & 315 & $247(100)$ & 8 & JWH-018 & 342 & $155(100)$ & 24 & AM1220 & 383 & $98(100)$ & 32 \\
\hline CP-47,497 C8 & 315 & $149(16)$ & 24 & JWH-018 & 342 & $214(25)$ & 20 & AM1220 & 383 & $155(25)$ & 24 \\
\hline CP-47,497 C8 & 315 & $121(57)$ & 24 & JWH-018 & 342 & $127(93)$ & 56 & AM1220 & 383 & $112(82)$ & 20 \\
\hline JWH-251 & 320 & $\mathbf{1 0 5}(100)$ & 24 & JWH-019 & 356 & $155(100)$ & 24 & JWH-200 & 385 & $\mathbf{1 5 5}(100)$ & 20 \\
\hline JWH-251 & 320 & $119(9)$ & 24 & JWH-019 & 356 & $228(21)$ & 24 & JWH-200 & 385 & $114(76)$ & 28 \\
\hline JWH-251 & 320 & $214(63)$ & 24 & JWH-019 & 356 & $127(92)$ & 56 & JWH-200 & 385 & $127(50)$ & 60 \\
\hline JWH-203 & 340 & $125(100)$ & 32 & & & & & & & & \\
\hline JWH-203 & 340 & $214(15)$ & 24 & & & & & & & & \\
\hline JWH-203 & 340 & $188(15)$ & 16 & & & & & & & & \\
\hline
\end{tabular}


VITA

\section{SEONGSHIN GWAK}

Born in Yesan, Republic of Korea

2002-2009

Bachelor of Science, Advanced Material Chemistry

Korea University

Sejong, Republic of Korea

2003-2005

Sergeant, Republic of Korea Army

2009-2011

Master of Science, Forensic Science

Sam Houston State University

Huntsville, Texas

2010-2011

Forensic Science Student Research Grant Award National Institute of Justice and the Forensic Sciences Foundation

Huntsville, Texas

2011-2015

Doctoral Candidate

Florida International University

Miami, Florida

2015

Third Place for Oral Presentation

Florida International University Scholarly Forum

Miami, Florida

2015

Dissertation Year Fellowship

Florida International University

Miami, Florida

\section{PUBLICATIONS AND SELECTED PRESENTATIONS}

Gwak, S. and Almirall, J. (2015). Rapid screening of 35 new psychoactive substances by ion mobility spectrometry (IMS) and direct analysis in real time (DART) coupled to QTOF-MS. Drug Testing and Analysis, DOI:10.1002/dta.1783.

Gwak, S., Arroyo-Mora, L. and Almirall, J. (2015). Qualitative analysis of seized synthetic cannabinoids and synthetic cathinones by gas chromatography triple quadrupole tandem mass spectrometry. Drug Testing and Analysis, 7(2), 121-130. 
Seongshin Gwak and Jose R. Almiral, Rapid Detection of 'Flakka' ( $\alpha$-PVP) by Ion Mobility Spectrometry (IMS) and Identification by Direct Analysis in Real Time Quadrupole Time-of-Flight (DART-QTOF) and Gas Chromatography Quadrupole Timeof-Flight (GC-QTOF), Presented at 4th Annual IFRI Forensic Science Symposium, May 2015, Miami, Florida.

Seongshin Gwak and Jose R. Almirall, Qualitative Analysis of New Psychoactive Substances by Ion Mobility Spectrometry and Direct Analysis in Real Time Quadrupole Time-of-Flight, Presented at 2015 ASMS Sanibel Conference, January 2015, Clearwater beach, Florida.

Seongshin Gwak and Jose R. Almirall, Qualitative Analysis of Emerging Designer Drugs by Gas Chromatography Tandem Mass Spectrometry, Ion Mobility Spectrometry, and Direct Analysis in Real Time Quadrupole Time-of-Flight, Presented at $20^{\text {th }}$ World Meeting of the International Association of Forensic Sciences, October 2014, Seoul, Republic of Korea.

Seongshin Gwak and Jose R. Almirall, Rapid Characterization of MDMA Analogues by Direct Analysis in Real Time Quadrupole Time-of-Flight, Presented at 62nd ASMS Conference on Mass Spectrometry and Allied Topics, June 2014, Baltimore, Maryland.

Seongshin Gwak and Jose R. Almirall, Differentiation of Synthetic Cathinone and Syntehtic Cannabinoid Regioisomers by GC-QQQ-MS/MS, Presented at AAFS 66th Annual Scientific Meeting, February 2014, Seattle, Washington.

Seongshin Gwak, Luis E. Arroyo-Mora, and Jose R. Almirall, Separation and Identification of Synthetic Cathinones using GC/MS, GC-MS/MS and ESI-IMS-MS, Presented at AAFS 65th Annual Scientific Meeting, February 2013, Washington, D.C.

Seongshin Gwak, Oliver Spicer, and Jose R. Almirall, Rapid Analysis of Synthetic Cathinones using Electrospray Ionization-Ion Mobility Spectrometry-Mass Spectrometry, Presented at $21^{\text {st }}$ International Conference on Ion Mobility Spectrometry, July 2012, Orlando, Florida.

Howard Holness, Seongshin Gwak and Jose Almirall, Chiral Separation of Amphetamine Type Substances Using Ion Mobility-Mass Spectrometry, Presented at 1st IFRI Forensic Science Symposium, March 2012, Miami, Florida.

Seongshin Gwak, Ayodele A. Collins, Cade park, Scot Chester, and James A. Bourland, Prevalence of Norhydrocodone in Authentic Hydrocodone Urine Specimens, Presented at AAFS 63rd Annual Scientific Meeting, February 2011, Chicago, Illinois. 